\title{
The effect of spin-spin interactions on nucleon-nucleus scattering
}

A thesis submitted to the Faculty of Engineering and Physical Sciences of the University of Surrey for the degree of Doctor of Philosophy

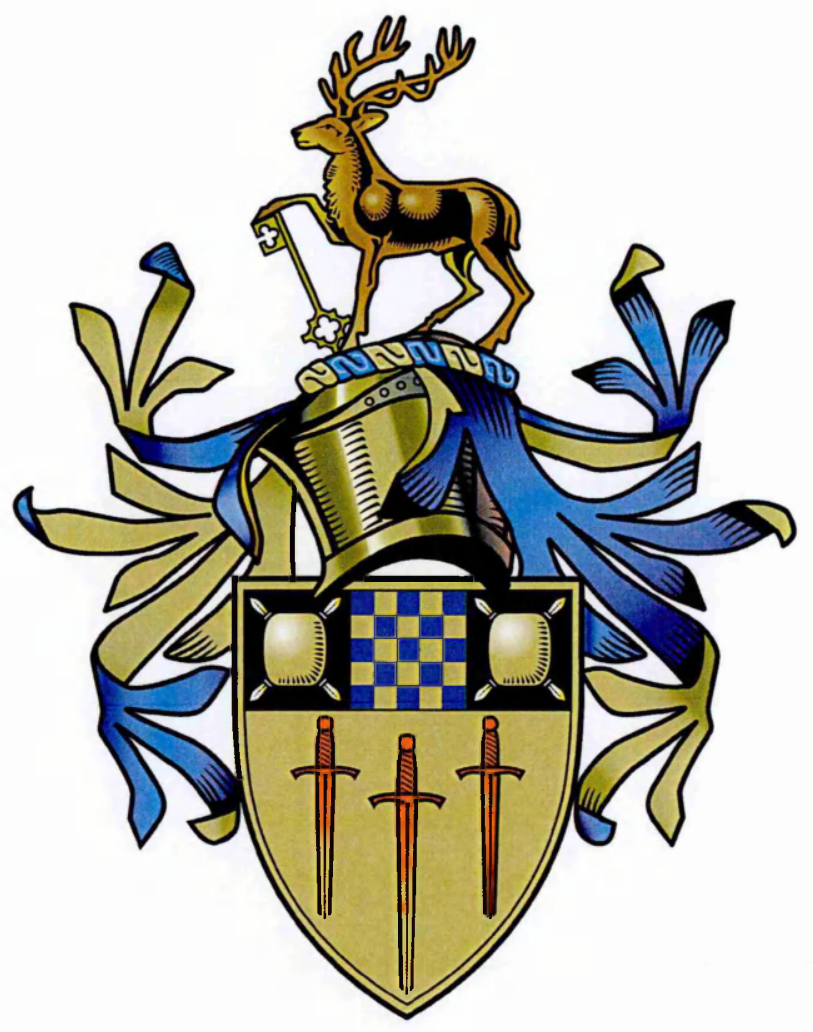

\section{Elizabeth Sarah Cunningham}

Department of Physics

Faculty of Engineering and Physical Sciences

University of Surrey

September 2010 
ProQuest Number: 13803900

All rights reserved

INFORMATION TO ALL USERS

The quality of this reproduction is dependent upon the quality of the copy submitted.

In the unlikely event that the author did not send a complete manuscript and there are missing pages, these will be noted. Also, if material had to be removed, a note will indicate the deletion.

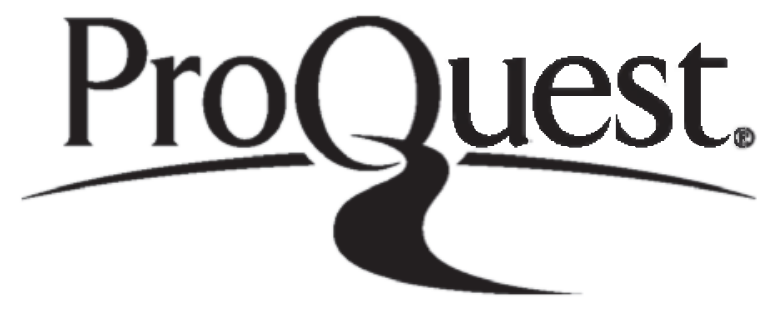

ProQuest 13803900

Published by ProQuest LLC (2018). Copyright of the Dissertation is held by the Author.

All rights reserved.

This work is protected against unauthorized copying under Title 17, United States Code Microform Edition (C) ProQuest LLC.

ProQuest LLC.

789 East Eisenhower Parkway

P.O. Box 1346

Ann Arbor, Ml 48106 - 1346 


\section{Abstract}

In this thesis we investigate the interactions between nucleons, which have spin- $1 / 2$, and nuclei, which may also have intrinsic spin. The dependence of the nucleon-nucleon interaction on the relative orientations of the spin of the two particles is well known and suggests a similar dependence may be present in the interactions of nucleons with nuclei. The effect of the spin of the nucleus on the nucleon-nucleus potential has been the subject of much theoretical and experimental interest. Previous work has found that in many cases spin-spin interactions cannot be ignored, especially for high spin exotic nuclei such as those likely to be produced in the next generation of radioactive beam facilities.

In this work a model has been developed for determining spin-spin terms in the nucleon-nucleus optical potential. These terms are constructed by folding a realistic effective nucleon-nucleon interaction, which includes both direct and exchange parts, over the nuclear wavefunction. The target is assumed, for our purposes, to have a simple two valence-particle structure, with the total spins of the two valence nucleons coupling to give the total spin of the nucleus. The resulting spin-spin tensors are evaluated within Distorted Wave Born Approximation to obtain the spin-spin amplitudes.

This general model has been used for the specific calculation of the elastic scattering of protons by ${ }^{10} \mathrm{~B}$, for which relevant experimental data exist. New spin-spin interactions that have higher order couplings of the target and projectile spins are included. In addition, where previous work on spin-spin interactions has only included exchange corrections for the central parts of the nucleon-nucleon interaction, we have included the non-local exchange contribution from the tensor parts of the nucleon-nucleon interaction. The effect of these spin-spin interactions on observables sensitive to the spin polarisation of the incident beam, specifically the polarisation transfer coefficient $D_{N N}$, has been investigated. We have found that the spin-spin interactions derived from this model have a significant effect on $D_{N N}$ when used to examine the elastic scattering of protons from ${ }^{10} \mathrm{~B}$. The calculation of $D_{N N}$ has also been shown to be particularly sensitive to the parameters of the model used to describe the nuclear structure. 


\section{Acknowledgements}

First of all I would like to thank my supervisors Jim Al-Khalili and Ron Johnson for the considerable amount of time, help, support and guidance they have given me over the past four years. Their instruction and encouragement is in every page of this work. Jim, no matter what was going on in your hectic schedule you always made time to see me regularly, thank you for being there for me. Ron, there isn't enough room in this thesis for me to be able to thank you for everything you have done for me, both as a teacher and as a friend, so I hope you know how grateful I am. I feel very privileged to have been able to work with both of you as your $\mathrm{PhD}$ student. I would also like to thank all the other member of the nuclear theory group at the University of Surrey, especially Jeff Tostevin and Paul Stevenson for many helpful discussions and nudges in the correct direction. Next I would like to give thanks to our collaborator Ed Stephenson for alerting us to this problem in the first place, for all his help along the way and also to him and his wife, Linda, for so warmly inviting me into their home when I was far from my own, thank you.

I have made a lot of new friends over the past four years to whom I owe a great debt of gratitude for keeping me sane and laughing during the darkest times of my $\mathrm{PhD}$. Gemma-love, your friendship is the most precious thing I have gained over the past four years. To my office mates Emma and Ed, keep living the dream. Ed, I'm sorry we had to leave you. Emma, hurry up, we need to drink that bottle very soon. Julian, I'm glad we got to become friends before you left, luckily you didn't wander too far away. Amy thank you for making the beginning of my journey easier. To the rest of the Park Barn Massive, Ian and Amanda and to Jeff, who will always be number one, thank you. Vinicio, thank you for making lunchtimes so 'interesting', I wish I could see myself through your eyes! To the theory boys, James, Arnau and David while you were not all here at the same time you have provided a constant stream of help, fun and friendship. To the lads of the nuclear experimental offices, past and present, Greg, Pete, Simon, Matt, Bendy, Dangerous, Bruce, Chris, Ryan and Steven Ashley who always made me laugh and always made me grateful to be a theorist! I hope I managed to put a little joy back in your lives too (if only through the regimented eating of cake). 
I wish also to thank my family. To my parents, thank you for starting me out on this path, even though you didn't quite understand it. You have always believed in me and given me freely everything I have ever needed. For this I will always be grateful. Rachel, you have always showered me with so much love and encouragement. If I had been able to choose anyone to be my little sister, I would still have picked you. Ean, thank you for your friendship and for making my sister so happy. I look forward to you becoming the brother I never had. Grandpa, your faith has always been a tremendous source of strength for me, I hope I've made you proud. Nanny, I wish I could show you what I have achieved, I miss you, but I know that you know.

Lastly I would like to thank my boyfriend Alex, not only for his unwavering love and support during my $\mathrm{PhD}$, but for giving me whatever I need before I know I need it. I've been told that surviving a $\mathrm{PhD}$ is a pretty good test of a relationship and you passed with flying colours (if only I could have done so well). It is not an exaggeration to say that I could not have done this without you. I love you my darling boy.

For the first six months of this year I have been a pretty bad girlfriend, daughter, sister, granddaughter and friend. Thank you all for your patience, I promise to do better now.

A charming young student called Liz

Thought that doing C-Gs was a wiz.

But from Racah's confection

She needed protection,

And found herself all in a tis!

Ron Johnson, February 2008 


\section{For Alex}

It's all for you...

"Consider the lilies of the field, how they grow; they toil not, neither do they spin." Matthew vi. 28. 


\section{Declaration of Originality}

This thesis and the work to which it refers are the results of my own efforts. Any ideas, data, images or text resulting from the work of others (whether published or unpublished) are fully identified as such within the work and attributed to their originator in the text, bibliography or in footnotes. This thesis has not been submitted in whole or in part for any other academic degree or professional qualification. I agree that the University has the right to submit my work to the plagiarism detection service TurnitinUK for originality checks. Whether or not drafts have been so-assessed, the University reserves the right to require an electronic version of the final document (as submitted) for assessment as above.

Elizabeth Cunningham 


\section{Contents}

1 Introduction $\quad 1$

1.1 Motivation ........................... 1

1.2 Previous Work on Spin-Spin Interactions . . . . . . . . . . . . . . 2

1.3 Polarisation Transfer Coefficient Measurements . . . . . . . . . . . 3

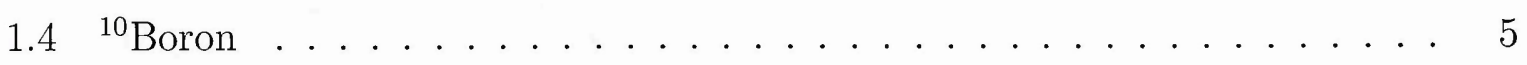

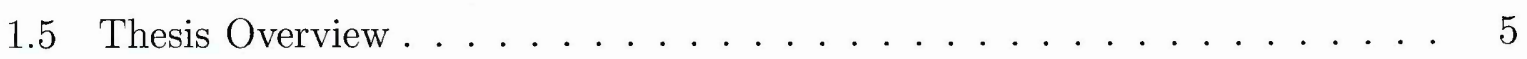

2 Spin-Spin Interactions $\quad 8$

2.1 Spin-Spin Operator $\boldsymbol{S}_{k_{I} k} \ldots \ldots \ldots \ldots \ldots$

2.1 .1 General Spin Operators $\tau_{k q}(\boldsymbol{S}) \ldots \ldots . \ldots . \ldots 9$

2.1.2 Explicit form of $\boldsymbol{S}_{k_{I}} \ldots \ldots \ldots \ldots \ldots$

2.2 Spin-Spin Amplitudes . . . . . . . . . . . . . . . . . 11

2.2.1 Spin-Spin Amplitude Checks . . . . . . . . . . . . . . . . . 14

2.3 Total Scattering Amplitude . . . . . . . . . . . . . . 17

3 Folding Model $\quad 19$

3.1 Generalised Optical Potential . . . . . . . . . . . . . 19

3.2 Folded Potential . . . . . . . . . . . . . . . . 20

3.3 Nuclear Wavefunction ... . . . . . . . . . . . . . 21

3.3.1 Valence Particle Wavefunction . . . . . . . . . . . . . . 22

3.3.2 Particles and Holes . . . . . . . . . . . . . . . . . . 23

3.4 General Folding . . . . . . . . . . . . . . . . . . . . 24

3.4.1 Multipole Expansion Coefficients . . . . . . . . . . . . 26

3.5 Central NN Interaction . . . . . . . . . . . . . . . . 26

3.5.1 Spin-Spin Potentials for Central Term . . . . . . . . . . . . 27

3.6 Tensor NN Interaction . . . . . . . . . . . . . . . . . . . 28

3.6.1 Folding Equation for all Components of the Tensor NN Interaction 28

3.6.2 Spin-Spin Potentials for Tensor Term 1 . . . . . . . . . . . . 29

3.6.3 Spin-Spin Potentials for Tensor Term 2 . . . . . . . . . . . . 30 
3.6.4 Spin-Spin Potentials for Tensor Term 3 . . . . . . . . . . . . . 31

3.6.5 Spin-Spin Potentials for All Tensor Terms . . . . . . . . . . . . 32

3.7 Analytic $\mu$ Integration . . . . . . . . . . . . . . . . . . 33

3.8 Spin-Orbit Terms . . . . . . . . . . . . . . . . . 33

3.9 Folding Checks . . . . . . . . . . . . . . . . . 34

3.9.1 Checks of Folded Potentials from Central NN Term . . . . . . . . . 34

3.9.2 Checks of Folded Potentials from Tensor NN Term . . . . . . . . . 36

4 Nucleon-Nucleon Interaction $\quad 40$

4.1 Effective Nucleon-Nucleon Interaction . . . . . . . . . . . . . . . 40

4.2 Spin- and Isospin- Structure of the Effective NN Interaction . . . . . . . . 42

4.3 Parametrisation . . . . . . . . . . . . . . . . . . 44

4.4 Exchange Terms . . . . . . . . . . . . . . . . 44

4.5 Zero-Range Pseudo-Potentials . . . . . . . . . . . . . 46

4.5.1 Central Exchange . . . . . . . . . . . . . 46

4.5 .2 Tensor Exchange . . . . . . . . . . . . . . . . 47

4.5 .3 Approximation for Q ..................... 48

5 Exchange Folding Model $\quad 51$

5.1 General Folding for Exchange Terms . . . . . . . . . . . . . 51

5.2 Central Exchange NN Interaction . . . . . . . . . . . . . . . 52

5.2.1 Spin-Spin Potentials for Central Exchange Term . . . . . . . . . . 52

5.3 Tensor Exchange NN Interaction . . . . . . . . . . . . . . 53

5.3.1 Spin-Spin Potentials for Tensor Exchange Term A . . . . . . . . . 56

5.3.2 Spin-Spin Potentials for Tensor Exchange Term B . . . . . . . . . 57

5.3.3 Spin-Spin Potentials for Tensor Exchange Term C . . . . . . . . . . 59

5.4 Modified Scattering Amplitudes . . . . . . . . . . . . . . 61

5.4 Tensor Term B . . . . . . . . . . . . . . . 62

5.4 .2 Tensor Term C . . . . . . . . . . . . . . . 63

5.5 Exchange Folding Checks . . . . . . . . . . . . . . . . 64

5.5.1 Checks of Folded Potentials from Central Exchange NN Term . . . 64

5.5.2 Checks of Folded Potentials from Tensor Exchange NN Term . . . . 65

5.5.3 Spin-Spin Potentials for Tensor Exchange Term i . . . . . . . . . . 67

5.5.4 Spin-Spin Potentials for Tensor Exchange Term ii . . . . . . . . . . 69

5.5.5 Spin-Spin Potentials for Tensor Exchange Term iii . . . . . . . . . . 70

5.5.6 Spin-Spin Potentials for Tensor Exchange Term iv . . . . . . . . . . 72

5.5.7 Modified Scattering Amplitudes . . . . . . . . . . . . . . . . . . 74

5.5 .8 Time-Reversal Invariance . . . . . . . . . . . . . . . . 75 
6 Spin-spin Interactions and Observables $\quad 79$

6.1 Single-Particle Wavefunction . . . . . . . . . . . . . 79

6.2 Direct Spin-Spin Interactions . . . . . . . . . . . . . . . 81

6.2.1 Spherical Spin-Spin Interaction . . . . . . . . . . . . . 84

6.2 .2 All Spin-Spin Interactions (Direct terms) . . . . . . . . . . . . . . . . . . . . . . . . . . . . .

6.3 Central Exchange Spin-Spin Interaction . . . . . . . . . . . . . . 90

6.4 Tensor Exchange Spin-Spin Interaction . . . . . . . . . . . . . . 93

6.4.1 Total NN Exchange Tensor Interaction . . . . . . . . . . . . . 95

6.4 .2 Effect of even $k_{I} \ldots \ldots \ldots \ldots$

6.5 All Spin-Spin Interactions and Q Dependence . . . . . . . . . . . . 101

6.6 Sensitivity of $D_{N N}$ to $u_{\ell j}(r) \ldots \ldots \ldots \ldots$. . . . . . . . . . . . . . . . . . . . .

6.7 Comparison with Experiment . . . . . . . . . . . 106

6.8 Computer Codes . . . . . . . . . . . . . . . 107

7 Conclusions and Outlook $\quad 109$

7.1 Conclusions . . . . . . . . . . . . . . . . . 109

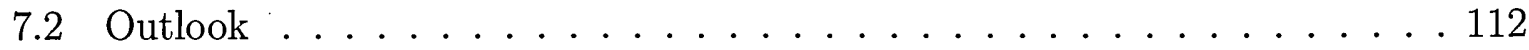

A Angular Momentum 114

A.1 Clebsch-Gordan Coefficient . . . . . . . . . . . . . . . . 114

A.2 Racah Coefficient . . . . . . . . . . . . . . . 115

A.3 9-j Symbol . . . . . . . . . . . . . . . . . 116

A.4 Spherical Harmonics . . . . . . . . . . . . . . . 117

B Simple Calculation of $D_{N N} \quad 119$

C Folded Potential Formalism: Direct Terms 121

C.1 Folding of Central NN interaction Term . . . . . . . . . . . . . . 121

C.2 Folding of Tensor NN Interaction Term . . . . . . . . . . . . . . 123

C.2.1 Form of the Tensor Potential . . . . . . . . . . . . . . . . 123

C.3 Folding of Tensor Term 1 . . . . . . . . . . . . . . . . . 124

C.4 Folding of Tensor Term 2 . . . . . . . . . . . . 126

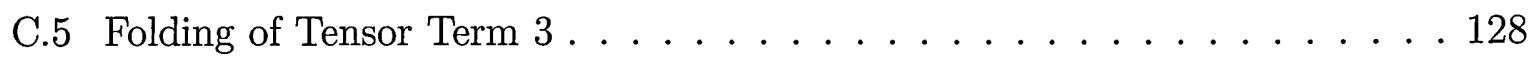

D Folded Potential Formalism: Exchange Terms $\quad 131$

D.1 Folding of Central Exchange NN Interaction Term . . . . . . . . . . . . . 131

D.2 Folding of Tensor Exchange NN Interaction Term . . . . . . . . . . . . . 132

D.3 NN Tensor Exchange Term A Folding Formalism . . . . . . . . . . . . . . 134

D.4 NN Tensor Exchange Term B Folding Formalism . . . . . . . . . . . . . 136 
D.5 NN Tensor Exchange Term C Folding Formalism . . . . . . . . . . 137

$\begin{array}{ll}\text { E Nucleon-Nucleon Interaction Parameters } & 141\end{array}$ 


\section{List of Figures}

3.1 The folding model coordinate system. . . . . . . . . . . . . 25

6.1 The $1 p_{\frac{3}{2}}$ single-particle wavefunctions . . . . . . . . . . . 80

6.2 Spin-spin form factors from the folding of the direct $v_{N N}^{\sigma \sigma}(r) \boldsymbol{\sigma}_{a} \cdot \boldsymbol{\sigma}_{b}$ term in the NN interaction, for elastic proton scattering from ${ }^{10} \mathrm{~B}$. . . . . . . 82

6.3 Spin-spin form factors from the folding of the direct $v_{N N}^{t r}(r) \boldsymbol{S}_{a b}$ term in the $\mathrm{NN}$ interaction, for elastic proton scattering from ${ }^{10} \mathrm{~B} \ldots \ldots . . . . .83$

6.4 Amplitudes and cross section calculation, without spin-orbit distorting potential, for elastic proton scattering from ${ }^{10} \mathrm{~B} \ldots \ldots \ldots$. . . . . . 85

6.5 Calculation of $D_{N N}$ from spherical spin-spin interaction, without spin-orbit distorting potential, for elastic proton scattering from ${ }^{10} \mathrm{~B} \ldots$. . . . . . 86

6.6 Ratio of the deviation of $D_{N N}$ from unity for the spherical spin-spin interaction $F_{10}(R)$ and $0.5 F_{10}(R)$, without spin-orbit distorting potential, for elastic proton scattering from ${ }^{10} \mathrm{~B} \ldots \ldots \ldots$. . . . . . . . 87

6.7 Cross section calculation, with spin-orbit distorting potential, for elastic proton scattering from ${ }^{10} \mathrm{~B} \ldots \ldots \ldots . \ldots . \ldots 88$

6.8 Calculation of $D_{N N}$ from spherical spin-spin interaction, with spin-orbit distorting potential, for elastic proton scattering from ${ }^{10} \mathrm{~B} \ldots \ldots . . .288$

6.9 Ratio of the deviation of $D_{N N}$ from unity for the spherical spin-spin interaction $F_{10}(R)$ and $0.5 F_{10}(R)$, with spin-orbit distorting potential, for elastic proton scattering from ${ }^{10} \mathrm{~B} \ldots \ldots \ldots . \ldots . \ldots . \ldots 9$

$6.10 D_{N N}$ calculated using all spin-spin interactions from folding of direct terms in the $\mathrm{NN}$ interaction, for elastic proton scattering from ${ }^{10} \mathrm{~B}$. . . . . . 90

6.11 Spin-spin form factors from the folding of the exchange $\hat{v}_{N N}^{\sigma \sigma}(r) \sigma_{a} \cdot \sigma_{b} P_{a b}^{r}$ term in the $\mathrm{NN}$ interaction, for elastic proton scattering from ${ }^{10} \mathrm{~B}$. . . . 91

$6.12 D_{N N}$ calculated using all spin-spin interactions from folding of central exchange term in the $\mathrm{NN}$ interaction, for elastic proton scattering from ${ }^{10} \mathrm{~B} .93$

6.13 Spin-spin form factors from the folding of tensor exchange Term A, for elastic proton scattering from ${ }^{10} \mathrm{~B} \ldots \ldots \ldots . \ldots . \ldots 96$ 
6.14 Spin-spin form factors from the folding of tensor exchange Term B, for elastic proton scattering from ${ }^{10} \mathrm{~B} \ldots \ldots . \ldots . \ldots 97$

6.15 Spin-spin form factors from the folding of tensor exchange Term $\mathrm{C}$, for elastic proton scattering from ${ }^{10} \mathrm{~B} \ldots \ldots . \ldots . \ldots 98$

$6.16 D_{N N}$ calculated using all spin-spin interactions from folding of tensor exchange terms in the NN interaction, for elastic proton scattering from ${ }^{10} \mathrm{~B} .99$

6.17 Angular dependence of exchange coefficients $\hat{J}_{\sigma \sigma}(Q)$ and $\hat{J}_{t r}(Q) \ldots \ldots 2$

$6.18 D_{N N}$ calculated using all spin-spin interactions from folding of direct and exchange central and tensor terms in the $\mathrm{NN}$ interaction, for elastic proton

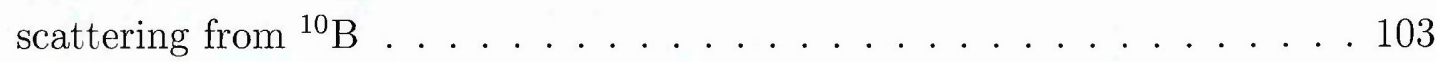

6.19 Ratio of the deviation of $D_{N N}$ from unity including all the spin-spin interactions $F_{k_{I} k}(R)$ and $0.5 F_{k_{I} k}(R)$, for elastic proton scattering from ${ }^{10} \mathrm{~B}$. . . 104

6.20 Woods-Saxon $1 p_{\frac{3}{2}}$ single-particle wavefunction, first and second derivatives with Max and Min geometry parameters $R_{W S}=(0.88 \pm 0.13)(A-1)^{1 / 3} \mathrm{fm}$

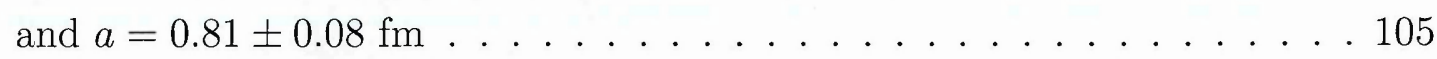

6.21 Calculation of $D_{N N}$, for elastic proton scattering from ${ }^{10} \mathrm{~B}$ using WoodsSaxon single-particle wavefunction with Max and Min geometry parameters $R_{W S}=(0.88 \pm 0.13)(A-1)^{1 / 3} \mathrm{fm}$ and $a=0.81 \pm 0.08 \mathrm{fm} \ldots \ldots . . . .106$

6.22 Calculation of $D_{N N}$, for elastic proton scattering from ${ }^{10} \mathrm{~B}$ using using four different single particle wavefunctions compared to the experimental measurement ....................... 107

A.1 The graphical representation of a C-G coefficient of the form $\left(j_{1} m_{1} j_{2} m_{2} \mid J M\right) .115$

A.2 The graph of the Racah coefficient, W(abcd;ef) . . . . . . . . . . 116

A.3 The graph of the $9 \mathrm{j}$ coefficient shown in Eq. A.10 . . . . . . . . . 117

C.1 The graphical representation of the C-G coefficients in Eq. C.19. . . . . . . 128

C.2 The graphical representation of the C-G coefficients in Eq. C.24. . . . . . . 130

D.1 The graphical representation of the C-G coefficients in Eq. D.15 . . . . . 135

D.2 The graphical representation of the C-G coefficients in Eq. D.22 . . . . . . 139 


\section{List of Tables}

2.1 Optical Model Parameters. . . . . . . . . . . . . . . . . . . . 17

AM Summary of notation for spin-spin potentials . . . . . . . . . 77

6.1 The volume integral checks of the spin-spin interactions calculated from the folding of the direct central term in the NN interaction. . . . . . . . 81

6.2 The volume integral checks of the spin-spin interactions calculated from the folding of the direct tensor term in the NN interaction. . . . . . . . . 84

6.3 The volume integral checks of the spin-spin interactions calculated from the folding of the central exchange term in the NN interaction. . . . . . . 92

6.4 Exchange coefficients $\hat{J}_{\sigma \sigma}(Q)$ and $\hat{J}_{t r}(Q)$ for $\theta=0$ and $\theta=90 . \ldots \ldots 101$

E.1 Real nucleon-nucleon interaction parameters . . . . . . . . . . . . . 141

E.2 Imaginary nucleon-nucleon interaction parameters . . . . . . . . . . . . 142 


\section{Chapter 1}

\section{Introduction}

\subsection{Motivation}

The study of nuclear reactions is mainly concerned with the scattering and reactions of a nuclear projectile, interacting through the strong and electromagnetic forces, with a target nucleus. Nuclear reactions and nuclear scattering are used to study the fundamental properties of nuclei, but they are not only of academic interest. Numerous applications of this field are also found in industry, medicine and energy production. Nuclear reactions also play an important role in astrophysics; research into stellar burning cycles and nucleosynthesis requires knowledge and understanding of nuclear reactions.

Elastic scattering is one of the simplest types of nuclear reaction. In this channel the projectile, $A$, and the target, $B$, start in their ground states and their final states are the same as their initial states. This is written as $B(A, A) B$. The kinetic energy of the relative motion of the two particles is asymptotically conserved, but their direction of motion changes. Despite the simplicity of this reaction, elastic scattering is an important source of information on nuclear properties.

The potential which is used to describe elastic scattering is the optical potential. In the optical model, the many-body problem arising from all the interactions between the nucleons in the target nucleus and those in the incident particle is encompassed in an effective two body interaction. The numerous nucleon-nucleon interactions are replaced by a single potential between the projectile and target. It is referred to as the optical model because it is analogous to the scattering of light. For example, in optics the various interactions of light with each particle in the medium is approximated using the refractive index of the medium. This work takes a careful look at the optical potential, or more specifically, its spin dependence.

With the next generation of radioactive beam facilities currently under construction there is a vital need for nuclear reaction theory calculations to make predictions of ob- 
servables relevant to the proposed experiments. In order to do this for the exotic nuclear species that will be produced in these facilities, it is necessary to determine accurate optical models to describe nucleon elastic scattering from targets with non-zero spin. This is one of the goals of this work.

\subsection{Previous Work on Spin-Spin Interactions}

The two-body nucleon-nucleon (NN) interaction contains a component which depends on the relative orientation of the spins of the two nucleons. For a non spin-zero nucleus a similar component in the nucleon-nucleus interaction may also be expected. Inclusion of terms in the optical potential which depend on the spin operator, $I$, of the target nucleus, were first proposed by Feshbach over 50 years ago [1,2]. Initially, many attempts were made to describe these spin-spin interactions using a central, or so-called 'spherical spin-spin' term of the form

$$
U_{S S}^{0}(R)=-V_{S S} F_{0}(R) \sigma_{0} \cdot \boldsymbol{I}
$$

where $R$ is the projectile target separation, $V_{S S}$ is the strength of the potential, $F_{0}(R)$ is the form factor and $\sigma_{0}$ is the Pauli spin operator for the scattered nucleon. Some early work [3-6] used a phenomenological optical model potential in which the spin-spin interactions were added and determined by a fit to experimental data. Others evaluated the spherical spin-spin potential microscopically from appropriate terms in the NN interaction [7-11].

Later works [12-14] included a second-rank 'tensor spin-spin' term given by

$$
U_{S S}^{T}(\boldsymbol{R})=-V_{S T} F_{T}(R)\left[3\left(\boldsymbol{\sigma}_{0} \cdot \hat{\boldsymbol{R}}\right)(\boldsymbol{I} \cdot \hat{\boldsymbol{R}})-\boldsymbol{\sigma}_{0} \cdot \boldsymbol{I}\right]
$$

which is analogous to the tensor force in the NN interaction. In each case, the strength of the tensor spin-spin potential was estimated phenomenologically. Most of these works were restricted by the limited amount of experimental data available for observables sensitive to these spin-spin terms and all found the strengths of these potentials to be small.

Two main types of optical model calculations, which include these spin-spin terms, have been carried out. The first of these treats the spin-spin potentials to first order in the framework of Distorted Wave Born Approximation (DWBA) [3, 8, 12-14] and, more recently, in $[15,16]$. These take advantage of the supposed weakness of the strength of the spin-spin interactions relative to the conventional optical potential terms such as central and spin-orbit. The second type are coupled channel calculations in which the scattering amplitude is handled in the channel spin framework [4,6,7] and, more recently, in $[17,18]$.

A good review of the early work on spin-spin interactions has been written by Sherif 
[19]. The author finishes by stating that 'improved microscopic calculations of the spinspin potentials, particularly the tensor term, will certainly be of great help in tracking down this ever evasive part of the nuclear optical potential'.

The work of McAbee [20,21] first attempted to derive a generalised spin-spin operator of the form shown in the next chapter. Microscopic calculations of the spin-spin potentials were performed using a valence-nucleon model for the target nucleus and an effective NN interaction. The formalism was developed for the folding of the direct central, spinorbit and tensor two body interaction terms over the nuclear wavefunction. A central exchange term was also included. The spin-spin amplitudes were calculated in DWBA although not all contributions were included in the final calculation of the observables and no attempts were made to calculate exchange terms for the spin-orbit or tensor NN interactions. McAbee found that in many cases the spin-spin interactions should not be neglected, especially for high spin exotic nuclei, such as those likely to be produced in the next generation of radioactive beam facilities.

\subsection{Polarisation Transfer Coefficient Measurements}

A useful observable when investigating the existence of spin-spin interactions is the depolarisation parameter, or polarisation transfer coefficient, $D$, (also known as the Wolfenstein $D$ parameter [22]). A nucleon-nucleus optical potential with no spin-spin interactions produces no depolarisation, i.e. $D=1$. Only terms in the optical potential which depend on the nucleus target-spin, $\boldsymbol{I}$, lead to a depolarisation which deviates from unity.

The first theoretical calculation of the depolarisation including spin-spin terms was carried out by Stamp [7]. He concluded that 'the simplest method of experimentally verifying the existence of a spin-spin interaction would be to measure the depolarisation'. Stamp specifically recommended measurements at angles corresponding to minima in the differential cross section. It is at these angles that the contributions from the standard terms in the optical potential are relatively small and the effect of the spin-spin interaction on the cross section is at a maximum. Therefore at these angles $D$ should have its greatest deviations from unity [6]. Unfortunately, errors in Stamp's derivation led to a large overestimation of the deviation of $D$ from unity at the first minimum [23]. This led experimentalists to concentrate their measurements of $D$ on this first cross section minimum $[24,25]$.

The first experiment measuring the angular distribution of $D$ was performed by the Tokyo group [26]. They measured large deviations of $D$ from unity for $1.36 \mathrm{MeV}$ neutrons scattering from ${ }^{27} \mathrm{Al}(I=5 / 2),{ }^{59} \mathrm{Co}(I=7 / 2),{ }^{63,65} \mathrm{Cu}(I=3 / 2)$ and ${ }^{209} \mathrm{Bi}(I=9 / 2)$ and they found $D=1$ for spin-zero $\mathrm{Ni}$. Other measurements of the angular distribution of $D$ for 
low energy protons scattering from non spin-zero nuclei [27-29], and references in [19], also show large deviations of $D$ from unity.

Attempts to fit this data using spherical and tensor spin-spin interactions were performed by $[6,13,27,30]$. However, there were uncertainties in the spin-spin interactions derived from the depolarisation data because deviations of $D$ from unity can also be caused by:

a) compound nucleus effects which cause large contributions at energies, below $\sim 30 \mathrm{MeV}[26,31]$;

b) the quadrupole spin-flip ${ }^{1}$ effects [32] that exist in the scattering from targets with spin greater than $1 / 2$.

For further discussion of these see [19].

More recent measurements, free from the contribution of compound nucleus effects, have been performed [33]. They indicate that the measured $D$ could not be accounted for by quadrupole spin-flip alone and spherical and tensor spin-spin interactions were needed to get a reasonable account of the data. More recent work [15,34] free from both effects indicated for the first time the existence of the spin-spin interactions unambiguously.

The experimental measurement of $D$ to be analysed in this work is detailed in Betker et al. [35]. They measure the polarization transfer coefficient, $D_{N N}$, (which is the same as the Wolfenstein $D$ parameter [22]) for $200 \mathrm{MeV}$ protons elastically scattering from ${ }^{10} \mathrm{~B}$ at the Indiana University Cyclotron Facility (IUCF). They attempt to account for the large deviation of $D_{N N}$ from unity, at large angles, using coupled channel calculations which include the spin-flip contributions to the scattering (but they do not include spin-spin interactions). Their calculation does well for cross section and analyzing power data and for $D_{N N}$ at forward angles where its deviation from unity is small. However at larger angles their calculation fails to reproduce the experimental data for $D_{N N}$. One of the goals of this work is to determine if the different types of spin-spin interactions have a significant effect on $D_{N N}$.

$D_{N N}$ (or $D_{y y}$ as it is sometimes called, where the direction of the y-axis is normal to the scattering plane), measures the extent to which the y-component of the incident proton polarisation is transferred to the outgoing proton polarisation. So, for $D_{N N}=$ 1 , the $y$-component of the outgoing proton polarisation is the same as the y-component of the incident proton polarisation. $D_{N N}$ will always remain equal to one unless a term dependent on the orientation of the target spin is present in the potential.

$D_{N N}$ is related to the total nucleon-nucleus scattering amplitude $\mathrm{F}$ (which will be defined in Sections 2.2 and 2.3), and the y-component $\sigma_{0 y}$, of the projectile nucleon spin

\footnotetext{
${ }^{1}$ Quadrupole spin-flip refers to the angular momentum $k_{I}$, transferred to the target nucleus during scattering. $k_{I}=0$, for the conventional optical potential, $k_{I}=1$, for the spherical and tensor spin-spin terms and $k_{I}=2$ for quadrupole spin-flip. This type of term is discussed in Chapter 5
} 
operator $\sigma_{0}$, by the following relation [36]:

$$
D_{N N}=\frac{\operatorname{Tr}\left[F \sigma_{0 y} F^{\dagger} \sigma_{0 y}\right]}{\operatorname{Tr}\left[F F^{\dagger}\right]},
$$

where the trace is taken over the projectile and target spin projections.

\section{$1.4 \quad{ }^{10}$ Boron}

If the projectile and target are both spin- $\frac{1}{2}$ then the spherical and tensor spin-spin interactions given in Eqs. 1.1 and 1.2 are the only local spin-spin operators allowed by parity, time reversal and angular momentum conservation. The odd- $\mathrm{N}$, odd-Z, ${ }^{10} \mathrm{~B}$ nucleus has a $3^{+}$ground state spin and parity [37-39]. This means that in order to calculate $D_{N N}$ for protons elastically scattering from ${ }^{10} \mathrm{~B}$ higher order terms are required (see Chapters 3 and 5).

In the simplest single-particle shell-model description, the ${ }^{10} \mathrm{~B}$ nucleus is a half-filled $1 p$-shell. In this work we will take our model of ${ }^{10} \mathrm{~B}$ to be a full $1 s_{1 / 2}$ shell with a proton and neutron hole in the $1 p_{\frac{3}{2}}$ shell. Using this simplistic nuclear wavefunction is consistent with previous works that microscopically derive the spin-spin interactions from the two-body interaction between an incident nucleon and a single valence target nucleon [7-10,16,20].

There are also works $[15,17]$ that investigate spin-spin interactions using more realistic nuclear wavefunctions from the work of Cohen and Kurath [40] for $1 p$-shell nuclei. The calculation in Betker et al. [35], which tried to explain their measurement of $D_{N N}$ without spin-spin interactions, used the more recent work on $1 p$-shell nuclei of Lee and Kurath [41] and ${ }^{10} \mathrm{~B}$ models from [37].

The simple single-particle shell-model approach used in this work will still enable a comprehensive investigation of spin-spin interactions, and the importance of the different rank tensor terms to the deviation of $D_{N N}$ from unity. However, this simple description of the nucleus will be a limiting factor when comparing the final calculation with the experimental data and an important improvement desirable for future work.

\subsection{Thesis Overview}

This thesis describes the development of target spin-dependent (spin-spin) terms, in the optical potential for elastic nucleon-nucleus scattering. Spin-spin operators and their associated potentials are evaluated within a folding model, in which a two valence-particle description of the target nucleus is assumed. A realistic nucleon-nucleon interaction, containing direct and exchange terms, is used to generate the spin-spin interactions, which 
are then treated in DWBA to calculate the polarisation transfer coefficient $D_{N N}$, for 200 $\mathrm{MeV}$ protons elastically scattered from ${ }^{10} \mathrm{~B}$.

Having introduced previous work on spin-spin interactions and described the observable $D_{N N}$, Sections 1.3 and 1.4 give the details of the measurement of $D_{N N}$ and the nucleus that will be examined in this work.

In Chapter 2, the general spin-spin operator, (constructed from the couplings of $\sigma_{0}$, $\boldsymbol{I}$ and the angular dependence of $\hat{\boldsymbol{R}}$ ), is defined along with the form of the spin-spin interactions. The spin-spin amplitude of this residual interaction is then evaluated using DWBA. The phenomenological optical potential used for the interaction (excluding spinspin terms) between the projectile nucleon and the target is described in Section 2.3, along with the total nucleon-nucleus elastic scattering amplitude.

Chapters 3 and 5 describe how the spin-spin interactions are constructed using the folding model and a two valence-nucleon description of the target nucleus. These chapters only contain an outline of the extensive formalism required to evaluate the spin-spin interactions and their form factors. The details of the Racah algebra techniques required to deal with the complicated angular momentum couplings associated with the generalised spin-spin tensors are given in Appendices C and D.

The realistic effective $\mathrm{NN}$ interaction used in the microscopic folding model to derive the spin-spin interactions is detailed in Chapter 4. The NN interaction is obtained from the updated Bonn-B potential using the Brueckner G-matrix approach as described in Section 4.1. The sum of Yukawa terms fitted to reproduce the G-matrix elements are calculated for free NN scattering at a projectile lab energy of $200 \mathrm{MeV}$, (the Yukawa parameters are given in Appendix E). Only the central and tensor terms in the NN interaction were used in the folding model. These give all the local spin-spin interactions that can be formed from the coupling of $\sigma_{0}, \boldsymbol{I}$ and $\boldsymbol{R}$. Spin-spin interactions from the folding of the spin-orbit term in the NN interaction are discussed in Section 3.8, although they are not included in this work.

The exchange corrections for the central and tensor $\mathrm{NN}$ interactions are performed using single-nucleon knock-on exchange terms, which are approximated using the zerorange pseudo-potentials described in Section 4.5. This work therefore contains the first attempt to incorporate the effects of tensor exchange on spin-spin interactions. The coefficients of the exchange pseudo-potentials have a dependence on the magnitude of the sum of the initial and final wave numbers. The approximations for this quantity are discussed in Section 4.5.3.

This thesis describes how the folding of a realistic NN interaction over the nuclear wavefunction has led to new spin-spin tensor interactions which go beyond the work of McAbee [20,21]. Higher order couplings of the target and projectile spins, which 
have never been examined before, are included. In addition, where exchange corrections included in previous work on spin-spin interactions have been limited to the central parts of the NN interaction, we have included the non-local exchange contribution from the tensor parts of the NN interaction. Chapter 6 shows that these new spin-spin interactions are important and should not be neglected when calculating the polarisation transfer coefficient, $D_{N N}$, for $\mathrm{p}+{ }^{10} \mathrm{~B}$ elastic scattering.

The conclusions of the research and recommendations for future work are discussed in Chapter 7. In the first Appendix the angular momentum formalism used throughout this work is defined. Appendix B describes a simple calculation of $D_{N N}$, in which the spinorbit interaction in the nucleon-nucleus interaction is set to zero and only the spherical spin-spin interaction is included. 


\section{Chapter 2}

\section{Spin-Spin Interactions}

In this chapter the generalised spin-spin operator used extensively in this work will be defined along with the form of the spin-spin potential. This enables the derivation of the DWBA spin-spin amplitude, which together with the scattering amplitude calculated from the nucleon-nucleus interactions (not including spin-spin) can be used to calculate observables such as $D_{N N}$.

\section{$2.1 \quad$ Spin-Spin Operator $S_{k_{I} k}$}

As discussed in Section 1.2, the calculation of local spin-spin interactions, before the work of McAbee [20,21], had been limited to two spin-spin operators denoted by $\boldsymbol{S}_{k_{I} k}$, where $k_{I}$ is the rank of the spin operator constructed from the components of the target spin $\boldsymbol{I}$ and $k$ is the rank of the spherical harmonic $Y_{k}(\hat{\boldsymbol{R}})$, of the unit vector of the projectiletarget separation. The first of these two operators, $S_{10}$, is proportional to $\sigma_{0} \cdot I$, while the second-rank tensor term, $\boldsymbol{S}_{12}$, is proportional to $\left[3\left(\boldsymbol{\sigma}_{0} \cdot \hat{\boldsymbol{R}}\right)(\boldsymbol{I} \cdot \hat{\boldsymbol{R}})-\boldsymbol{\sigma}_{0} \cdot \boldsymbol{I}\right]$. If the projectile and target both have spin- $\frac{1}{2}$ these are the only local spin-spin operators allowed by parity, time reversal and angular momentum conservation. For target nuclei with higher spin, spin-spin interactions of higher ranks are allowed (see Chapter 3).

McAbee $[20,21]$ was the first to discuss a general spin-spin operator and did not limit his calculation of spin-spin interactions to just the spherical and tensor terms. The generalised local spin-spin operator used in this work is given by

$$
\boldsymbol{S}_{k_{I} k}=\left[\tau_{1}\left(\boldsymbol{\sigma}_{0}\right) \times \tau_{k_{I}}(\boldsymbol{I})\right]_{k} \cdot Y_{k}(\hat{\boldsymbol{R}}),
$$

which is analogous to that of Eq. 2.2 in McAbee [20]. The components of Eq. 2.1 are a spherical harmonic $Y_{k}(\hat{\boldsymbol{R}})$ as described in [42] and Appendix A.4 and the standard spin operators, $\tau_{1 q_{0}}\left(\boldsymbol{\sigma}_{0}\right)$ and $\tau_{k_{I} q_{I}}(\boldsymbol{I})[42,43]$, for the projectile and target spins respectively, 
which have matrix elements

$$
\begin{aligned}
& \left\langle\frac{1}{2} \mu_{0}^{\prime}\left|\tau_{1 q_{0}}\left(\boldsymbol{\sigma}_{0}\right)\right| \frac{1}{2} \mu_{0}\right\rangle=\sqrt{3}\left(\frac{1}{2} \mu_{0} 1 q_{0} \mid \frac{1}{2} \mu_{0}^{\prime}\right), \\
& \left\langle I M^{\prime}\left|\tau_{k_{I} q_{I}}(\boldsymbol{I})\right| I M\right\rangle=\hat{k}_{I}\left(I M k_{I} q_{I} \mid I M^{\prime}\right) \text {, }
\end{aligned}
$$

where $\hat{k}_{I}$ is used to denote $\sqrt{2 k_{I}+1}$ and the properties of the Clebsch-Gordan (C-G) coefficients $\left(\frac{1}{2} \mu_{0} 1 q_{0} \mid \frac{1}{2} \mu_{0}^{\prime}\right)$ and $\left(I M k_{I} q_{I} \mid I M^{\prime}\right)$ are given in [42] and Appendix A.1.

\subsubsection{General Spin Operators $\tau_{k q}(\boldsymbol{S})$}

The operators $\tau_{1 q_{0}}\left(\boldsymbol{\sigma}_{0}\right)$ and $\tau_{k_{I} q_{I}}(\boldsymbol{I})$ are constructed out of the $x, y$ and $z$ components of their spin operators $\boldsymbol{\sigma}_{0}$ and $\boldsymbol{I}$ respectively. For a general spin, $\boldsymbol{S}$, the relationships between the spin operator $\tau_{k q}(\boldsymbol{S})$ and the components $S_{x}, S_{y}$ and $S_{z}$ can be derived using the following expression derived from Eqs. 1d and 1e in Hooton and Johnson [43]

$$
\hat{k}_{1} \hat{k}_{2} \hat{S} W\left(I k_{2} I k_{1} ; I k_{3}\right) \tau_{k_{3} q_{3}}(\boldsymbol{S})=\sum_{q_{1} q_{2}}\left(k_{2} q_{2} k_{1} q_{1} \mid k_{3} q_{3}\right) \tau_{k_{1} q_{1}}(\boldsymbol{S}) \tau_{k_{2} q_{2}}(\boldsymbol{S})
$$

and the relations between components of vector tensors in the spherical and Cartesian coordinate systems

$$
\begin{aligned}
\tau_{1 \pm 1}(\boldsymbol{S}) & =\mp \frac{1}{\sqrt{2}} C(S)\left[S_{x} \pm \imath S_{y}\right] \\
\tau_{10}(\boldsymbol{S}) & =C(S) S_{z}
\end{aligned}
$$

The spin dependent coefficient $C(S)$ is the same for all $\tau_{1 q}(\boldsymbol{S})$ because they rotate as spherical harmonics (Eq. 1c [43]). $C(S)$ can be determined using the matrix element of $\tau_{10}(\boldsymbol{S})$

$$
\begin{aligned}
\left\langle S S\left|\tau_{10}(S)\right| S S\right\rangle & =\sqrt{3}(S S 10 \mid S S)=C(S)\left\langle S S\left|S_{Z}\right| S S\right\rangle \\
& =\sqrt{3} \sqrt{\frac{S}{S+1}}=C(S) S \\
\rightarrow C(S) & =\sqrt{\frac{3}{S(S+1)}}
\end{aligned}
$$

Therefore

$$
\tau_{1 \pm 1}(\boldsymbol{S})=\mp \frac{1}{\sqrt{2}} \sqrt{\frac{3}{S(S+1)}}\left[S_{x} \pm \imath S_{y}\right] \quad ; \quad \tau_{10}(\boldsymbol{S})=\sqrt{\frac{3}{S(S+1)}} S_{z}
$$


Using Eq. 2.4 with $k_{3}=2$ and $k_{1}=k_{2}=1$ leads to

$$
\begin{aligned}
\tau_{2 \pm 2}(\boldsymbol{S}) & =\frac{3}{S(S+1)} \frac{1}{6 \hat{S} W(S 1 S 1 ; S 2)}\left[S_{x} \pm \imath S_{y}\right]^{2} \\
\tau_{2 \pm 1}(\boldsymbol{S}) & =\mp \frac{3}{S(S+1)} \frac{1}{6 \hat{S} W(S 1 S 1 ; S 2)}\left[S_{z}\left(S_{x} \pm \imath S_{y}\right)+\left(S_{x} \pm \imath S_{y}\right) S_{z}\right] \\
\tau_{20}(\boldsymbol{S}) & =\frac{3}{S(S+1)} \frac{1}{3 \sqrt{6} \hat{S} W(S 1 S 1 ; S 2)}\left[3 S_{z}^{2}-S(S+1)\right]
\end{aligned}
$$

This method can be used to calculate all $\tau_{k q}(\boldsymbol{S})$ in terms of the $x, y$ and $z$ components of their spin $S$. However, for this work it is far more convenient to use the spin operator $\tau_{k q}(\boldsymbol{S})$. Discussion of the spin-spin operator $\boldsymbol{S}_{k_{I} k}$ in terms of the projectile and target spin operators $\sigma_{0}$ and $\boldsymbol{I}$ will be limited the spherical and tensor spin-spin terms to allow comparison with previous work.

\subsubsection{Explicit form of $S_{k_{I} k}$}

Using C-G coefficients the general spin-spin operator can be written explicitly as

$$
\boldsymbol{S}_{k_{I} k}=\sum_{q_{0} q_{I} q}\left(1 q_{0} k_{I} q_{I} \mid k-q\right) \tau_{1 q_{0}}\left(\boldsymbol{\sigma}_{0}\right) \tau_{k_{I} q_{I}}(\boldsymbol{I}) Y_{k q}(\hat{\boldsymbol{R}})(-)^{q}
$$

The angular momentum coupling shown in the C-G coefficient of Eq. 2.10 requires that for the general spin-spin operator $\boldsymbol{S}_{k_{I} k}, k_{I}=k,|k \pm 1|$, because the projectile spin operator $\sigma_{0}$ is rank 1. The effects of parity transformation for the operators in Eq. 2.10 can be summarised as follows

$$
\begin{array}{cl}
I \rightarrow I & , \quad \sigma_{0} \rightarrow \sigma_{0} \\
\boldsymbol{R} & \rightarrow-\boldsymbol{R}
\end{array}
$$

and under time reversal

$$
\begin{aligned}
\boldsymbol{I} \rightarrow-\boldsymbol{I} & , \boldsymbol{\sigma}_{0} \rightarrow-\boldsymbol{\sigma}_{0} \\
\boldsymbol{R} & \rightarrow \boldsymbol{R} .
\end{aligned}
$$

Therefore in order for the generalised spin-spin operator to be invariant under parity and time-reversal, $k_{I}$ must be odd and $k$ must be even. This holds and is a useful check for all the local potentials considered in this work. Non-local interactions will be discussed in Chapter 5 when considering the tensor NN-interaction exchange terms.

The generalised spin-spin operator $S_{k_{I} k}$, can be reduced to give the spherical spin-spin 
operator. Taking $k_{I}=1$ and $k=0$ and using standard angular momentum recoupling techniques [42] leads to

$$
S_{10}=\frac{-\sigma_{0} \cdot \boldsymbol{I}}{\sqrt{4 \pi} \sqrt{I(I+1)}} .
$$

Different authors have used different normalisations when dealing with the spin-spin interactions so caution must be taken when comparing the strengths of the potentials.

Using the same techniques, the tensor spin-spin operator can be obtained from Eq. 2.10 by taking $k_{I}=1$ and $k=2$ to give

$$
\boldsymbol{S}_{12}=\frac{\sqrt{5}}{\sqrt{8 \pi} \sqrt{I(I+1)}}\left[3\left(\boldsymbol{\sigma}_{0} \cdot \hat{\boldsymbol{R}}\right)(\boldsymbol{I} \cdot \hat{\boldsymbol{R}})-\boldsymbol{\sigma}_{0} \cdot \boldsymbol{I}\right]
$$

In both cases the transition from the $\tau_{1 \mu_{0}}\left(\boldsymbol{\sigma}_{0}\right)$ and $\tau_{k_{I} q_{I}}(\boldsymbol{I})$ to the $\boldsymbol{\sigma}_{0}$ and $\boldsymbol{I}$ spin operators was made using Wigner-Eckart theorem [42]

$$
\begin{aligned}
\left\langle\frac{1}{2} \mu_{0}^{\prime}\left|\left(\sigma_{0}\right)_{1 q_{0}}\right| \frac{1}{2} \mu_{0}\right\rangle & =\left\langle\frac{1}{2}\left\|\sigma_{0}\right\| \frac{1}{2}\right\rangle\left(\frac{1}{2} \mu_{0} 1 q_{0} \mid \frac{1}{2} \mu_{0}^{\prime}\right), \\
\left\langle I M^{\prime}\left|(I)_{k_{I} q_{I}}\right| I M\right\rangle & =\left\langle I|| I^{k_{I}}|| I\right\rangle\left(I M k_{I} q_{I} \mid I M^{\prime}\right),
\end{aligned}
$$

and the following relations for their reduced matrix elements

$$
\begin{aligned}
\left\langle\frac{1}{2}\left\|\sigma_{0}\right\| \frac{1}{2}\right\rangle & =\sqrt{3} \\
\left\langle I\left\|I^{k_{I}}\right\| I\right\rangle & =k_{I} !\left[\frac{\left(2 I+k_{I}+1\right) !}{2^{k_{I}}(2 I+1)\left(2 k_{I}\right) !\left(2 I-k_{I}\right) !}\right]^{\frac{1}{2}},
\end{aligned}
$$

where the reduced matrix element for the target spin is taken from [17]. The use of the generalised spin-spin operator defined in Eqs. 2.1 and 2.10 will enable the calculation of a general scattering amplitude for each allowed combination of $k_{I}$ and $k$. This will be discussed in the next section.

\section{$2.2 \quad$ Spin-Spin Amplitudes}

Following on from previous work $[3,20]$ we shall consider spin-spin terms in the optical potential of the form

$$
U_{k_{I} k}(\boldsymbol{R})=F_{k_{I} k}(R) \boldsymbol{S}_{k_{I} k}
$$

where $U_{k_{I}}(\boldsymbol{R})$ are the spin-spin optical potentials and $F_{k_{I} k}(R)$ are the radial from factors.

At this point we will assume that the strengths of the spin-spin interactions are small when compared to the conventional $\boldsymbol{I}$-independent terms in the optical potential, so that the former can be treated in the Distorted Wave Born Approximation (DWBA). 
The DWBA is a first order approximation in which the interaction, $V(a, A)$, between the projectile, $a$, and target, $A$, is split into an auxiliary potential, $V_{0}$, which includes a large part of the effects of $V(a, A)$, and a residual interaction, $U$, that is much weaker and can be treated as a perturbation. Previous work indicates that the strength of the central spin-spin interaction $U_{10}(R)$ (of the form given in Eq. 1.1) is of the order $1 \mathrm{MeV}$ [18] and that a large tensor spin-spin potential $U_{12}(\boldsymbol{R})$ (of the form given in Eq. 1.2) has a strength of $2 \mathrm{MeV}$ [34]. The treatment of the spin-spin interactions as a residual interaction in the DWBA therefore seems a sensible starting point for the analysis of these spin-spin terms.

In DWBA, the scattering amplitudes for the nucleon elastic scattering into polar angles $(\theta, \phi)$ caused by the spin-spin potentials can be written as [44]

$$
f_{k_{I} k}\left(\mu_{f}, M_{f} ; \mu_{i}, M_{i} ; \theta, \phi\right)=\frac{-\mu_{p t}}{2 \pi \hbar^{2}}\left\langle\chi_{\mu_{f}}^{(-)}\left(\boldsymbol{R}, \boldsymbol{k}^{\prime}\right), I M_{f}\left|U_{k_{I} k}(\boldsymbol{R})\right| \chi_{\mu_{i}}^{(+)}(\boldsymbol{R}, \boldsymbol{k}), I M_{i}\right\rangle
$$

where $\chi_{\mu_{f}}^{(-)}\left(\boldsymbol{R}, \boldsymbol{k}^{\prime}\right)$ and $\chi_{\mu_{i}}^{(+)}(\boldsymbol{R}, \boldsymbol{k})$ are the final and initial distorted waves respectively with a final, $\mu_{f}$, and initial, $\mu_{i}$, incident nucleon spin projection. The final and initial spin projections of the nucleus are $M_{f}$ and $M_{i}$ respectively. The scattered and incident wave numbers are $\boldsymbol{k}^{\prime}$ and $\boldsymbol{k}$ and $\mu_{p t}$ is the reduced mass of the projectile-target system. The distorted wavefunction $\chi_{\mu_{i}}^{(+)}(\boldsymbol{R}, \boldsymbol{k})$ is a solution of the Schrödinger equation for the elastic scattering of the projectile on the target by the auxiliary potential, i.e. they are wavefunctions distorted from plane waves by the Coulomb and nuclear potentials (see Section 2.3) other than spin-spin. Using the notation of Satchler [44], $\chi_{\mu_{i}}^{(+)}(\boldsymbol{R}, \boldsymbol{k})$ can be written as

$$
\chi_{\mu_{i}}^{(+)}(\boldsymbol{R}, \boldsymbol{k})=4 \pi \sum_{\ell, m_{\ell}, j, m_{j}} e^{\imath \sigma_{\ell}} \frac{u_{\ell, j}\left(k_{c m} R\right)}{k_{c m} R}\left(\ell m_{\ell} \frac{1}{2} \mu_{i} \mid j m_{j}\right) Y_{\ell m_{\ell}}^{*}(\hat{\boldsymbol{k}}) \Phi_{\ell, \frac{1}{2}, j}^{m_{j}}(\hat{\boldsymbol{R}})
$$

this is equivalent to Eq. 4.48 in [44] for a projectile nucleon, with $e^{\imath \sigma_{\ell}} u_{\ell, j}\left(k_{c m} R\right)=$ $\chi_{\ell}^{j}\left(k_{c m}, R\right)$. In Eq. 2.21, $\sigma_{\ell}$ are the Coulomb partial-wave phase shifts, $u_{\ell, j}\left(k_{c m} R\right)$ are the distorted radial wavefunctions and $k_{c m}$ is the magnitude of the wave number in the centre of mass system. The spin-angle function $\Phi_{\ell, \frac{1}{2}, j}^{m_{j}}(\hat{\boldsymbol{R}})$ contains the coupling of the spherical harmonic and the projectile nucleon spin wavefunction, $\mathcal{X}_{\frac{1}{2} \mu}$

$$
\Phi_{\ell, \frac{1}{2}, j}^{m_{j}}(\hat{\boldsymbol{R}})=\sum_{m_{\ell}^{\prime}, \mu_{i}^{\prime}} \imath^{\ell}\left(\ell m_{\ell}^{\prime} \frac{1}{2} \mu_{i}^{\prime} \mid j m_{j}\right) \mathcal{X}_{\frac{1}{2} \mu_{i}^{\prime}} Y_{\ell m_{\ell}^{\prime}}(\hat{\boldsymbol{R}})
$$

Following the prescription in Section 4.3 .1 of [44] the time-reversed solution $\chi_{\mu_{f}}^{(-)}\left(\boldsymbol{R}, \boldsymbol{k}^{\prime}\right)$ 
is given by

$$
\chi_{\mu_{f}}^{(-)}\left(\boldsymbol{R}, \boldsymbol{k}^{\prime}\right)=4 \pi \sum_{\ell^{\prime} m_{\ell}^{\prime}, j^{\prime} m_{j}^{\prime}} e^{-\imath \sigma_{\ell^{\prime}}} \frac{u_{\ell^{\prime}, j^{\prime}}^{*}\left(k_{c m} R\right)}{k_{c m} R}\left(\ell^{\prime} m_{\ell}^{\prime} \frac{1}{2} \mu_{f} \mid j^{\prime} m_{j}^{\prime}\right) Y_{\ell^{\prime} m_{\ell}^{\prime}}^{*}\left(\hat{\boldsymbol{k}}^{\prime}\right) \Phi_{\ell^{\prime}, \frac{1}{2}, j^{\prime}}^{m_{j}^{\prime}}(\hat{\boldsymbol{R}}) .
$$

In order to calculate the scattering amplitude given by Eq. 2.20 the incident beam direction is chosen to be along the $\mathrm{z}$-axis. This choice simplifies $\chi_{\mu_{i}}^{(+)}(\boldsymbol{R}, \boldsymbol{k})$ to

$$
\chi_{\mu_{i}}^{(+)}(\boldsymbol{R})=\sqrt{4 \pi} \sum_{\ell, j, m_{j}} \hat{\ell} e^{\imath \sigma_{\ell}} \frac{u_{\ell, j}\left(k_{c m} R\right)}{k_{c m} R}\left(\ell 0 \frac{1}{2} \mu_{i} \mid j m_{j}\right) \Phi_{\ell, \frac{1}{2}, j}^{m_{j}}(\hat{\boldsymbol{R}}) .
$$

Now, substituting all these components into Eq. 2.20, including the form of the spin-spin operator $\boldsymbol{S}_{k_{I} k}$ from Eq. 2.10 gives

$$
\begin{aligned}
& f_{k_{I} k}\left(\mu_{f}, M_{f} ; \mu_{i}, M_{i} ; \theta, \phi\right)=\frac{-\mu_{p t}(4 \pi)^{3 / 2}}{2 \pi \hbar^{2}} \int d \boldsymbol{R} \sum_{\ell^{\prime}, m_{\ell}^{\prime}, j^{\prime}, m_{j}^{\prime}} \imath^{-\ell^{\prime}} e^{\imath \sigma_{\ell^{\prime}}} \frac{u_{\ell^{\prime}, j^{\prime}}\left(k_{c m} R\right)}{k_{c m} R}\left(\ell^{\prime} m_{\ell}^{\prime} \frac{1}{2} \mu_{f} \mid j^{\prime} m_{j}^{\prime}\right) Y_{\ell^{\prime} m_{\ell}^{\prime}}\left(\hat{\boldsymbol{k}}^{\prime}\right) \\
& \quad \times \sum_{m_{\ell}^{\prime \prime \prime}, \mu_{f}^{\prime}, m_{\ell}^{\prime \prime}, \mu_{i}^{\prime}}\left(\ell^{\prime} m_{\ell}^{\prime \prime \prime} \frac{1}{2} \mu_{f}^{\prime} \mid j^{\prime} m_{j}^{\prime}\right) Y_{\ell^{\prime} m_{\ell}^{\prime \prime \prime}}^{*}(\hat{\boldsymbol{R}}) F_{k_{I} k}(R) \sum_{q_{0} q_{I} q}\left(1 q_{0} k_{I} q_{I} \mid k-q\right) \\
& \quad \times\left\langle\frac{1}{2} \mu_{f}^{\prime}\left|\tau_{1 q_{0}}\left(\boldsymbol{\sigma}_{0}\right)\right| \frac{1}{2} \mu_{i}^{\prime}\right\rangle\left\langle I M_{f}\left|\tau_{k_{I} q_{I}}(\boldsymbol{I})\right| I M_{i}\right\rangle Y_{k q}(\hat{\boldsymbol{R}})(-)^{q} \sum_{\ell, j, m_{j}} \hat{\ell} \imath^{\ell} e^{\imath \sigma_{\ell}} \frac{u_{\ell, j}\left(k_{c m} R\right)}{k_{c m} R} \\
& \quad \times\left(\ell 0 \frac{1}{2} \mu_{i} \mid j m_{j}\right)\left(\ell m_{\ell}^{\prime \prime} \frac{1}{2} \mu_{i}^{\prime} \mid j m_{j}\right) Y_{\ell m_{\ell}^{\prime \prime}}(\hat{\boldsymbol{R}}) .
\end{aligned}
$$

Using Eq. A.17 in Appendix A.4 for the angular integration of the three spherical harmonics dependent on $\hat{\boldsymbol{R}}$ and Eqs. 2.2 and 2.3 for the matrix elements of the spin operators leads to the following

$$
\begin{aligned}
f_{k_{I} k}( & \left.\mu_{f}, M_{f} ; \mu_{i}, M_{i} ; \theta, \phi\right) \\
= & \frac{-\sqrt{3}}{E_{c m}} \sum_{\substack{\ell^{\prime}, m_{\ell}^{\prime}, j^{\prime}, m_{j}^{\prime} \\
\ell, j, m_{j}}} \hat{\ell}^{\ell-\ell^{\prime}} e^{\imath\left(\sigma_{\ell}+\sigma_{\ell^{\prime}}\right)}\left(\ell^{\prime} m_{\ell}^{\prime} \frac{1}{2} \mu_{f} \mid j^{\prime} m_{j}^{\prime}\right)\left(\ell 0 \frac{1}{2} \mu_{i} \mid j m_{j}\right) Y_{\ell^{\prime} m_{\ell}^{\prime}}\left(\hat{\boldsymbol{k}}^{\prime}\right) \\
& \times \int_{0}^{\infty} d R u_{\ell^{\prime}, j^{\prime}}\left(k_{c m} R\right) F_{k_{I} k}(R) u_{\ell, j}\left(k_{c m} R\right) \sum_{\substack{m_{\ell}^{\prime \prime \prime}, \mu_{f}^{\prime} \\
m_{\ell}^{\prime \prime}, \mu_{i}^{\prime}}}\left(\ell^{\prime} m_{\ell}^{\prime \prime \prime} \frac{1}{2} \mu_{f}^{\prime} \mid j^{\prime} m_{j}^{\prime}\right) \\
& \times\left(\ell m_{\ell}^{\prime \prime} \frac{1}{2} \mu_{i}^{\prime} \mid j m_{j}\right) \sum_{q_{0} q_{I} q}\left(1 q_{0} k_{I} q_{I} \mid k-q\right)\left(\frac{1}{2} \mu_{i}^{\prime} 1 q_{0} \mid \frac{1}{2} \mu_{f}^{\prime}\right) \hat{k}_{I}\left(I M k_{I} q_{I} \mid I M^{\prime}\right) \\
& \times(-)^{q} \frac{\hat{k} \hat{\ell}}{\hat{\ell}^{\prime}}\left(k q \ell m_{\ell} \mid \ell^{\prime} m_{\ell}^{\prime}\right)\left(k 0 \ell 0 \mid \ell^{\prime} 0\right),
\end{aligned}
$$

where the centre of mass projectile-target energy is $E_{c m}=k_{c m}^{2} \hbar^{2} / 2 \mu_{p t}$. The five $\mathrm{C}-\mathrm{G}$ 
coefficients in red can be reduced using Eq. A.12 which gives the relationship between C$\mathrm{G}$ coefficients and $9 \mathrm{j}$ symbols described in [42] and Appendix A.3. This reduction leads to the final equation for the spin-spin interaction DWBA scattering amplitude, or spin-spin amplitude

$$
\begin{aligned}
& f_{k_{I} k}\left(\mu_{f}, M_{f} ; \mu_{i}, M_{i} ; \theta, \phi\right) \\
& \quad=\frac{\sqrt{6}}{E_{c m}}(-)^{k_{I}+k-2 \mu_{i}+q_{I}} \hat{k_{I}} \hat{k}^{2} \sum_{\ell, \ell^{\prime}, j, j^{\prime}} \hat{\ell}^{2} \hat{j} \imath^{\ell-\ell^{\prime}} e^{\imath\left(\sigma_{\ell}+\sigma_{\ell^{\prime}}\right)}\left(\ell^{\prime} \mu_{i}-q_{I}-\mu_{f} \frac{1}{2} \mu_{f} \mid j^{\prime} \mu_{i}-q_{I}\right) \\
& \quad \times\left(\ell 0 \frac{1}{2} \mu_{i} \mid j \mu_{i}\right) Y_{\ell^{\prime}, \mu_{i}-q_{I}-\mu_{f}}\left(\hat{\boldsymbol{k}}^{\prime}\right) \int_{0}^{\infty} u_{\ell^{\prime}, j^{\prime}}\left(k_{c m} R\right) F_{k_{I} k}(R) u_{\ell, j}\left(k_{c m} R\right) d R \\
& \quad \times\left(k 0 \ell 0 \mid \ell^{\prime} 0\right)\left(I M_{i} k_{I} q_{I} \mid I M_{f}\right)\left(j \mu_{i} k_{I}-q_{I} \mid j^{\prime} \mu_{i}-q_{I}\right)\left\{\begin{array}{ccc}
\ell^{\prime} & \frac{1}{2} & j^{\prime} \\
\ell & \frac{1}{2} & j \\
k & 1 & k_{I}
\end{array}\right\},
\end{aligned}
$$

where combinations of the projectile and target spin projections have been used for $m_{\ell}^{\prime}$, $m_{j}, m_{j}^{\prime}$ and $q_{I}=M_{f}-M_{i}$. Eq. 2.27 is analogous to Eq. 2.22 in McAbee [20].

To simplify the calculation of the scattering amplitude again, the y-axis is chosen to be perpendicular to the scattering plane, along $\boldsymbol{k} \times \boldsymbol{k}^{\prime}$, so that $\phi=0$. In this case the symmetry relation [44]

$$
f_{k_{I} k}\left(-\mu_{f},-M_{f} ;-\mu_{i},-M_{i} ; \theta, 0\right)=(-)^{\mu_{i}+M_{i}-\mu_{f}-M_{f}} f_{k_{I} k}\left(\mu_{f}, M_{f} ; \mu_{i}, M_{i} ; \theta, 0\right),
$$

can be used if the $\boldsymbol{S}_{k_{I} k}$ are parity-invariant and $k$ is even.

\section{Relativistic Calculation}

In the final calculation for $200 \mathrm{MeV}$ protons elastically scattering from ${ }^{10} \mathrm{~B}$ a relativistic energy momentum relation was used. The prescription used to describe potential scattering of relativistic particles is taken from Section 6.8 of [45] and discussed for elastic deuteron-nucleus scattering in $[46,47]$.

\subsubsection{Spin-Spin Amplitude Checks}

Evaluating Eq. 2.27 when the spin-orbit term in the main optical potential is set to zero $[12,21]$, can be used as a check of the calculation of the scattering amplitude. This means there is no interaction in the distorting potential dependent on the projectile spin, 
which reduces the scattering amplitude in Eq. 2.27 to

$$
\begin{aligned}
f_{k_{I} k}^{n o L S} & \left(\mu_{f}, M_{f} ; \mu_{i}, M_{i} ; \theta, \phi\right) \\
& =-\frac{\sqrt{3}}{E_{c m}}\left(1 \mu_{f}-\mu_{i} k_{I} q_{I} \mid k \mu_{f}+q_{I}-\mu_{i}\right)\left(I M_{i} k_{I} q_{I} \mid I M_{f}\right)\left(\frac{1}{2} \mu_{i} \quad 1 \mu_{f}-\mu_{i} \mid \frac{1}{2} \mu_{f}\right) \\
& \times \hat{k_{I}} \hat{k}(-)^{\mu_{i}-q_{I}-\mu_{f}} \sum_{\ell, \ell^{\prime}} \frac{\hat{\ell}^{2}}{\hat{\ell}^{\prime}} l^{\ell-\ell^{\prime}} e^{\imath\left(\sigma_{\ell}+\sigma_{\ell^{\prime}}\right)} Y_{\ell^{\prime}, \mu_{i}-q_{I}-\mu_{f}}\left(\hat{\boldsymbol{k}}^{\prime}\right)\left(k 0 \ell 0 \mid \ell^{\prime} 0\right) \\
& \times\left(k \mu_{i}-q_{I}-\mu_{f} \ell 0 \mid \ell^{\prime} \mu_{i}-q_{I}-\mu_{f}\right) \int_{0}^{\infty} u_{\ell^{\prime}}\left(k_{c m} R\right) F_{k_{I} k}(R) u_{\ell}\left(k_{c m} R\right) d R .
\end{aligned}
$$

When there is no spin-orbit potential the distorted radial wavefunctions for a given $\ell$ no longer depend on $j$. This simplifies the reduction, as the sums over $j$ and $j^{\prime}$ may be performed using the orthogonality of the C-G coefficients (see Appendix A, Eq. A.1).

It is also useful to evaluate this amplitude for the central spin-spin potential only, i.e. $k_{I}=1$ and $k=0$. This simplification gives

$$
\begin{aligned}
f_{10}^{n o}{ }^{L S} & \left(\mu_{f}, M_{f} ; \mu_{i}, M_{i} ; \theta, 0\right) \\
& =\frac{\sqrt{3}}{\sqrt{4 \pi} E_{c m}} \sum_{\ell} \hat{\ell}^{2} e^{\imath 2 \sigma_{\ell}} P_{\ell}(\cos (\theta))(-)^{q_{I}}\left(I M_{i} 1 q_{I} \mid I M_{f}\right)\left(\frac{1}{2} \mu_{i} 1-q_{I} \mid \frac{1}{2} \mu_{f}\right) \\
& \times \int_{0}^{\infty} u_{\ell}\left(k_{c m} R\right)^{2} F_{10}(R) d R
\end{aligned}
$$

where $\theta$ is the scattering angle, the angle between the vectors $\boldsymbol{k}$ and $\boldsymbol{k}^{\prime}$ and $P_{\ell}(\cos \theta)$ is a Legendre Polynomial as described in [42]. Using Eqs. 2.15 - 2.18 for the matrix elements of $\sigma_{0}$ and $\boldsymbol{I}$, Eq. 2.30 becomes

$$
\begin{aligned}
f_{10}^{n o} L S(\theta) & =\frac{1}{\sqrt{4 \pi} E_{c m}} \sum_{\ell} \hat{\ell}^{2} e^{\imath 2 \sigma_{\ell}} P_{\ell}(\cos \theta) \int_{0}^{\infty} u_{\ell}\left(k_{c m} R\right)^{2} F_{10}(R) d R \frac{\boldsymbol{\sigma}_{0} \cdot \boldsymbol{I}}{\sqrt{I(I+1)}} \\
& =h_{10}(\theta) \frac{\boldsymbol{\sigma}_{0} \cdot \boldsymbol{I}}{\sqrt{I(I+1)}} .
\end{aligned}
$$

With this simplified spin-spin amplitude the whole nucleon-nucleus elastic scattering amplitude can now be written as

$$
F(\theta)=g(\theta) \mathbf{1}+h_{10}(\theta) \frac{\boldsymbol{\sigma}_{0} \cdot \boldsymbol{I}}{\sqrt{I(I+1)}},
$$

where $g(\theta)$ is the scattering amplitude for a spin-zero projectile and target, as discussed 
in [44] and Section 2.3. This amplitude can be substituted into Eq. 1.3 for $D_{N N}$ to give

$$
\begin{aligned}
D_{N N} & =\frac{\operatorname{Tr}\left[F \sigma_{0 y} F^{\dagger} \sigma_{0 y}\right]}{\operatorname{Tr}\left[F F^{\dagger}\right]}=\frac{|g(\theta)|^{2}-\frac{1}{3}\left|h_{10}(\theta)\right|^{2}}{|g(\theta)|^{2}+\left|h_{10}(\theta)\right|^{2}} \\
& =1-\frac{\frac{4}{3}\left|h_{10}(\theta)\right|^{2}}{|g(\theta)|^{2}+\left|h_{10}(\theta)\right|^{2}}
\end{aligned}
$$

where $\sigma_{0 y}$ is the y-component of the projectile spin operator $\sigma_{0}$ and the steps in Eq. 2.33 are expanded further in Appendix B. The trace, $T r$, is taken over the projectile and target spin projections.

Expressing $D_{N N}$ in this way shows that, $D_{N N}=1$, if there is no $\boldsymbol{\sigma}_{0} \cdot \boldsymbol{I}$ term in the scattering amplitude in Eq. 2.32 and that the presence of this spin-spin term means $D_{N N}<1$. It also reveals that not only is a spin-spin term dependent on $\boldsymbol{I}$ required for $D_{N N} \neq 1$ but it must be coupled to $\sigma_{0}$. If there is no dependence on $\sigma_{0}$ in the scattering amplitude, $\operatorname{Tr}\left[F \sigma_{0 y} F^{\dagger} \sigma_{0 y}\right]=\operatorname{Tr}\left[F F^{\dagger}\right]$ and $D_{N N}=1$. Eq. 2.33 also provides another useful check. When the $\boldsymbol{I}$-dependent term is much larger than the $\boldsymbol{I}$-independent term, i.e. $\left|h_{10}(\theta)\right|^{2}>>|g(\theta)|^{2}$, the observable $D_{N N} \rightarrow-1 / 3$.

\section{Plane Waves}

Another useful check of the calculation of the DWBA spin-spin amplitude is to set the main optical potential $V_{0}$, defined in the next section, to zero so the distorted waves become plane waves

$$
\chi_{\mu_{i}}^{(+)}(\boldsymbol{R}, \boldsymbol{k}) \stackrel{V_{0}(r) \rightarrow 0}{\longrightarrow} e^{\imath \boldsymbol{k} \cdot \boldsymbol{R}} \mathcal{X}_{\frac{1}{2} \mu_{i}}
$$

where $\mathcal{X}_{\frac{1}{2} \mu_{i}}$ is now a unit operator in spin space. The Rayleigh expansion of a plane wave is given by [48]

$$
e^{\imath \boldsymbol{k} \cdot \boldsymbol{R}}=4 \pi \sum_{\ell m_{\ell}} \imath^{\ell} j_{\ell}\left(k_{c m} R\right) Y_{\ell m_{\ell}}^{*}(\hat{\boldsymbol{k}}) Y_{\ell m_{\ell}}(\hat{\boldsymbol{R}})
$$

where $j_{\ell}\left(k_{c m} R\right)$ is a spherical Bessel function of the first kind [49]. Using this expansion instead of the distorted waves in Eq. 2.20 leads to a plane wave spin-spin amplitude and reduces Eq. 2.27 to

$$
\begin{aligned}
f_{k_{I} k}^{P W} & \left(\mu_{f}, M_{f} ; \mu_{i}, M_{i} ; \theta, \phi\right) \\
& =-\frac{\sqrt{3} k_{c m}^{2}}{E_{c m}} \hat{k_{I}} \hat{k} \sum_{\ell, \ell^{\prime}} \frac{\hat{\ell}^{2}}{\hat{\ell^{\prime}}} \imath^{\ell-\ell^{\prime}} Y_{\ell^{\prime}, \mu_{i}-q_{I}-\mu_{f}}\left(\hat{\boldsymbol{k}^{\prime}}\right) \int_{0}^{\infty} R^{2} j_{\ell^{\prime}}\left(k_{c m} R\right) F_{k_{I} k}(R) j_{\ell}\left(k_{c m} R\right) d R \\
& \times\left(1 \mu_{f}-\mu_{i} k_{I} q_{I} \mid k \mu_{f}+q_{I}-\mu_{i}\right)\left(\frac{1}{2} \mu_{i} 1 \mu_{f}-\mu_{i} \mid \frac{1}{2} \mu_{f}\right)\left(I M_{i} k_{I} q_{I} \mid I M_{f}\right)(-)^{\mu_{i}-q_{I}-\mu_{f}} \\
& \times\left(k \mu_{i}-q_{I}-\mu_{f} \ell 0 \mid \ell^{\prime} \mu_{i}-q_{I}-\mu_{f}\right)\left(k 0 \ell 0 \mid \ell^{\prime} 0\right),
\end{aligned}
$$


where $q_{I}=M_{f}-M_{i}$ and the incident beam direction is chosen to be along the z-axis. Calculations using plane wave amplitudes were carried out for all the spin-spin amplitudes in this work. These were then compared to calculations using the full DWBA amplitudes where the distorting potential was set to zero. Agreement was found in all cases.

\subsection{Total Scattering Amplitude}

A phenomenological optical potential is used for the interaction, not including spin-spin terms, between the projectile proton and the ${ }^{10} \mathrm{~B}$ nucleus and is of the form

$$
\begin{aligned}
V_{0}(R)= & V_{\text {Coul }}(R)+V f\left(R, r_{V}, a_{V}\right)+i W f\left(R, r_{W}, a_{W}\right)+V_{G} g\left(R, r_{G}, a_{G}\right) \\
& +2\left[V_{S O} \frac{1}{R} \frac{d}{d R} f\left(R, r_{S O R}, a_{S O R}\right)+i W_{S O} \frac{1}{R} \frac{d}{d R} f\left(R, r_{S O I}, a_{S O I}\right)\right] \boldsymbol{\ell} \cdot \sigma_{0},
\end{aligned}
$$

where the Woods-Saxon $f\left(R, r_{i}, a_{i}\right)$, and Gaussian $g\left(R, r_{i}, a_{i}\right)$ form factors are given by

$$
\begin{aligned}
& f\left(R, r_{i}, a_{i}\right)=\left(1+\exp \left[\left(R-r_{i} A^{1 / 3}\right) / a_{i}\right]\right)^{-1} \\
& g\left(R, r_{i}, a_{i}\right)=\exp \left[-\left(\frac{R-r_{i} A^{1 / 3}}{a_{i}}\right)^{2}\right]
\end{aligned}
$$

and $V_{\text {Coul }}(R)$ is the Coulomb potential generated by a uniformly charged sphere with a Coulomb radius of $\mathrm{R}_{\text {Coul }}=1.46 A^{1 / 3} \mathrm{fm}$. The parameters for this phenomenological potential are taken from [35] for $200 \mathrm{MeV}$ proton scattering from ${ }^{10} \mathrm{~B}$ and are given in Table 2.1. Optical potentials are discussed in more detail at the beginning of the next chapter.

The scattering amplitude obtained from this optical potential can be calculated exactly without need for the DWBA (for full details see Appendix A.2. of [44]). The total nucleon-

\begin{tabular}{|c||c|c|c|}
\hline Term & Depth & $r_{i}$ & $a_{i}$ \\
\hline \hline Real WS central & -8.9951 & 1.481 & 0.5808 \\
Imaginary WS central & -28.0739 & 0.8582 & 0.685 \\
Real Gaussian central & 9.0615 & 0.5378 & 0.8549 \\
Real WS Spin-Orbit & -3.8620 & 0.9323 & 0.644 \\
Imaginary WS Spin-Orbit & 0.65145 & 0.951 & 0.705 \\
\hline
\end{tabular}

Table 2.1: Optical model parameters. The Depths are in $\mathrm{MeV}$ and the geometry parameters are in $\mathrm{fm}$. 
nucleus elastic scattering amplitude matrix is then given by

$$
\begin{aligned}
F\left(\mu_{f}, M_{f} ; \mu_{i}, M_{i} ; \theta\right) & =g(\theta) \delta_{M_{f}, M_{i}} \delta_{\mu_{f}, \mu_{i}}+(-)^{\frac{1}{2}-\mu_{f}} h(\theta) \delta_{M_{f}, M_{i}} \delta_{\mu_{f},-\mu_{i}} \\
& +\sum_{k_{I} k} f_{k_{I} k}\left(\mu_{f}, M_{f} ; \mu_{i}, M_{i} ; \theta\right)
\end{aligned}
$$

where the functions $g(\theta)$ and $h(\theta)$ can be written as partial wave expansions in the following way [44]

$$
\begin{aligned}
& g(\theta)=f_{\text {Coul }}(\theta)+\frac{\imath}{2 k_{c m}} \sum_{\ell}\left[(2 \ell+1)-(\ell+1) S_{\ell}^{+}-\ell S_{\ell}^{-}\right] e^{2 \imath \sigma_{\ell}} P_{\ell}(\cos \theta), \\
& h(\theta)=\frac{\imath}{2 k_{c m}} \sum_{\ell}\left(S_{\ell}^{-}-S_{\ell}^{+}\right) e^{2 \imath \sigma_{\ell}} P_{\ell}^{1}(\cos \theta)
\end{aligned}
$$

where the superscripts \pm denote total angular momentum $j=\ell \pm \frac{1}{2}, S_{\ell}^{j}$ is the scattering matrix element for the $\ell$ th partial wave, which is unity for no scattering i.e. $V_{0}(r)=0$ and independent of $j$ if there is no spin-orbit interaction. The associated Legendre Polynomials $P_{\ell}^{1}(\cos \theta)$ are discussed in Appendix A.4 and the Rutherford amplitude $f_{\text {Coul }}(\theta)$ is

$$
f_{\text {Coul }}(\theta)=\frac{-\eta}{2 k_{c m} \sin ^{2}\left(\frac{1}{2} \theta\right)} \exp \left[-\imath \eta \ln \left\{\sin ^{2}\left(\frac{1}{2} \theta\right)\right\}+2 \imath \sigma_{0}\right]
$$

and $\eta$ is the standard Coulomb or Sommerfeld parameter. Eq. 2.40 is the full scattering amplitude used in Eq 1.3 to calculate $D_{N N}$ for $200 \mathrm{MeV}$ protons elastically scattered from ${ }^{10} \mathrm{~B}$. 


\section{Chapter 3}

\section{Folding Model}

The aim of this folding model is to derive microscopically the spin-spin potentials in terms of the interactions of the individual nucleons rather than using a phenomenological model. In order to do this two components are needed. The first is the nuclear wavefunction which describes the structure of the nucleus. As discussed in Section 1.4 the assumption of a simple single-particle shell model will be used for this part of the calculation. The second component required is the interaction between the projectile nucleon and the target nucleons. This element will be discussed in detail in Chapter 4.

\subsection{Generalised Optical Potential}

The Schrödinger equation can be used to consider the elastic scattering of a nucleon by a nucleus with $A$ nucleons

$$
(E-H) \Psi^{s c}=0,
$$

where $E$ is the energy in the centre of mass frame and the Hamiltonian $H$ is given by

$$
H=T+V+H_{0}(x),
$$

where $T$ is the total kinetic energy operator in the centre of mass frame, $V$ is the sum of nucleon-nucleon (NN) potentials between the projectile nucleon and each nucleon in the target and $H_{0}(x)$ is the internal Hamiltonian acting on the target nucleons with coordinates $x$. The total scattering wavefunction $\Psi^{s c}$ can be expanded in terms of a complete set of nuclear states $\Psi_{\alpha}^{A}$

$$
\Psi^{s c}=\sum_{\alpha} \xi_{\alpha} \Psi_{\alpha}^{A}
$$


summing over all the possible states of $\alpha$. The coefficient $\xi_{\alpha}$ describes the relative motion of the two particles in a state described by $\Psi_{\alpha}^{A}$. Using Feshbach's unified theory of nuclear reactions $[50,51]$ in terms of the projection operators $P$ and $Q$, which project on and off a selected set of open channels, obeying the relations

$$
P \Psi^{s c}=\xi_{0} \Psi_{0}^{A}, \quad Q \Psi^{s c}=(1-P) \Psi^{s c}
$$

the generalised optical potential can be expressed, following the method in Section 6.2 of [52], as

$$
U_{o p t}=\left(\Psi_{0}^{A}|V| \Psi_{0}^{A}\right)+\left(\Psi_{0}^{A}\left|V Q \frac{1}{E-H_{Q Q}} Q V\right| \Psi_{0}^{A}\right)
$$

where the round brackets imply summations and integration over the internal coordinates of the target nucleus and $H_{Q Q}=Q H Q$. The first term in Eq. 3.5 is called the folded potential and represents the interaction between the projectile nucleon and the target nucleus as the sum of all interactions between the projectile and target constituents averaged over the target ground state wavefunction. If exchange effects are not considered and the interaction potential $V$ is local, the term $\left(\Psi_{0}^{A}|V| \Psi_{0}^{A}\right)$, has the form $U(r)$, i.e. it is local in configuration space. The second term is often referred to as the dynamic polarisation potential and represents scattering through non-elastic intermediate excited states of the nucleus and causes the optical potential to be non-local in configuration space [52].

In phenomenological optical potentials $U_{\text {opt }}$ is approximated by a local potential $U(r)$ usually of Woods-Saxon form. This type of optical potential is used in this work for the interaction (other than spin-spin) between the projectile nucleon and target nucleus, see Section 2.3. In general, phenomenological optical potentials have real and imaginary parts and the imaginary parts describe the absorption effect due to nonelastic channels.

Instead of using a phenomenological model for the spin-spin interactions, in this work a folded potential is used as an approximation to the generalised optical potential, with $V$ taken to be a realistic NN interaction between the incoming nucleon and the target nucleons. The effective interaction used in this folding model goes a bit further than the first term in Eq. 3.5. Instead of using a $V$, a complex t-matrix is used which incorporates some of the effects of the second term of Eq. 3.5 (see Section 4.1). The importance of second order effects relevant to distorted wave calculations of nuclear scattering has been discussed in $[53,54]$.

\subsection{Folded Potential}

Since the initial work by Greenlees et al. [55], folding models have been widely used to generate potentials for use in nuclear scattering problems, see references in $[44,56]$. The 
development of realistic effective NN interactions (see Chapter 4) has led to the increased use of folding techniques in the analysis of scattering observables [57]. As discussed in the previous section, the folding integral in elastic scattering involves averaging the sum of NN interactions over the target ground state wavefunction

$$
\left(\Psi_{I, M_{f}}^{A}|V| \Psi_{I, M_{i}}^{A}\right)=\int \Psi_{I, M_{f}}^{* A}\left(\boldsymbol{r}_{1} \ldots \boldsymbol{r}_{A}\right) \sum_{i=1}^{A} V_{N N}(0, i) \Psi_{I, M_{i}}^{A}\left(\boldsymbol{r}_{1} \ldots \boldsymbol{r}_{A}\right) d \boldsymbol{r}_{1} \ldots d \boldsymbol{r}_{A}
$$

where $\Psi_{I, M}^{A}\left(\boldsymbol{r}_{1} \ldots \boldsymbol{r}_{A}\right)$ is the target nucleus ground state wavefunction and the two-body effective NN interaction $V_{N N}(0, i)$ between projectile nucleon 0 and target nucleon $i$ with separation, $r$, has the form

$$
V_{N N}(0, i)=v_{N N}^{c e}(r)+v_{N N}^{\sigma \sigma}(r) \sigma_{0} \cdot \sigma_{i}+v_{N N}^{l s}(r) \ell_{0 i} \cdot\left(\sigma_{0}+\sigma_{i}\right)+v_{N N}^{t r}(r) \boldsymbol{S}_{0 i}
$$

and will be discussed in further detail in Chapter 4.

Spin-spin interactions were first studied within folding models using a single valencenucleon description of the nucleus by Stamp [7] and then by Nagamine et al. [8]. These works only included contributions from the $v_{N N}^{\sigma \sigma}(r) \sigma_{0} \cdot \sigma_{i}$ term in the NN interaction and the potential $U_{10}$ (from Eq. 2.19) was the only spin-spin interaction discussed. Satchler $[9,10]$, went one step further including both $v_{N N}^{\sigma \sigma}(r) \sigma_{0} \cdot \sigma_{i}$ and $v_{N N}^{t r}(r) \boldsymbol{S}_{0 i}$ terms from the NN interaction, the folding of these gave the spin-spin potentials $U_{10}$ and $U_{12}$.

A thorough analysis of spin-spin potentials which result from the folding of all the terms in the NN interaction given in Eq. 3.7 has been given by Petrovich et al. [17] and McAbee [20,21]. Both performed a multipole decomposition of a NN interaction containing central, spin-orbit and tensor parts and the resulting expressions were folded over the target wavefunctions. Petrovich used this to derive a general expression for the optical potential for the elastic scattering of two non spin-zero nuclei and used coupledequations formalism to obtain the scattering observables. McAbee used this to derive the form factors for his generalised spin-spin operators, Eq. 2.19, which were then treated in the DWBA to obtain the spin-spin observables.

\subsection{Nuclear Wavefunction}

This work adopts a two valence-particle model for the nuclear wavefunction, thus reducing the A-body target wavefunction to a 3-body $($ core $+2 \mathrm{~N})$ one. It assumes the total spins the two valence nucleons $j_{1}$ and $j_{2}$ couple together to give the total spin $I$ of the nucleus and the pair orbit an inert spin-zero core. This assumption means the target nucleus ground state wavefunction can be expressed in terms of an uncoupled core wavefunction 
$\Phi_{\text {core }}\left(\boldsymbol{r}_{3} \ldots \boldsymbol{r}_{A}\right)$ and the wavefunction of the two valence particles $\Psi_{I, M}\left(\boldsymbol{r}_{1}, \boldsymbol{r}_{2}\right)$

$$
\Psi_{I, M}^{A}\left(\boldsymbol{r}_{1} \ldots \boldsymbol{r}_{A}\right) \simeq \Phi_{\text {core }}\left(\boldsymbol{r}_{3} \ldots \boldsymbol{r}_{A}\right) \Psi_{I, M}\left(\boldsymbol{r}_{1}, \boldsymbol{r}_{2}\right)
$$

The folded potential between the projectile, 0 , and the target nucleus, $\mathrm{A}$, is split into two components

$$
\begin{aligned}
\left(\Psi_{I, M_{f}}^{A}|V| \Psi_{I, M_{i}}^{A}\right) & =\left\langle\Phi_{\text {core }}\left(\boldsymbol{r}_{3} \ldots \boldsymbol{r}_{A}\right)\left|\sum_{i=3}^{A} V_{N N}(0, i)\right| \Phi_{\text {core }}\left(\boldsymbol{r}_{3} \ldots \boldsymbol{r}_{A}\right)\right\rangle \delta_{M_{i}, M_{f}} \\
& +\left\langle\Psi_{I, M_{f}}\left(\boldsymbol{r}_{1}, \boldsymbol{r}_{2}\right)\left|\sum_{i=1,2} V_{N N}(0, i)\right| \Psi_{I, M_{i}}\left(\boldsymbol{r}_{1}, \boldsymbol{r}_{2}\right)\right\rangle
\end{aligned}
$$

the first term and the non spin-spin components of the second term are the interaction between the projectile and spin-zero core, which has central and spin-orbit parts and is included through the phenomenological optical potential described in Section 2.3. The second term in Eq. 3.9 is the interaction between the projectile and valence nucleons. For the case of proton scattering from ${ }^{10} \mathrm{~B}$, the second term in Eq. 3.9 for valence particles 1 (proton) and 2 (neutron), can be expanded to give

$$
\left\langle\Psi_{I, M_{f}}\left(\boldsymbol{r}_{1}, \boldsymbol{r}_{2}\right)\left|V_{p p}(0,1)+V_{p n}(0,2)\right| \Psi_{I, M_{i}}\left(\boldsymbol{r}_{1}, \boldsymbol{r}_{2}\right)\right\rangle
$$

It is from this term that the target spin $I$ dependent spin-spin potentials will be derived in the following sections and chapters.

\subsubsection{Valence Particle Wavefunction}

The wavefunction for the valence nucleons is assumed to have the simple form [58]

$$
\Psi_{I, M}\left(\boldsymbol{r}_{1}, \boldsymbol{r}_{2}\right)=\left[\Phi_{\ell_{1} j_{1} m_{1}}\left(\boldsymbol{r}_{1}\right) \times \Phi_{\ell_{2} j_{2} m_{2}}\left(\boldsymbol{r}_{2}\right)\right]_{I M}
$$

where the valence particles, nucleon 1 and nucleon 2 are at a distance of $\boldsymbol{r}_{1}$ and $\boldsymbol{r}_{2}$ from the core respectively. The nucleon wavefunctions $\Phi_{\ell j m}(\boldsymbol{r})$ are defined as

$$
\Phi_{\ell j m}(\boldsymbol{r})=\sum_{\lambda m_{s}}\left(\ell \lambda \frac{1}{2} m_{s} \mid j m\right) u_{\ell j}(r) Y_{\ell \lambda}(\hat{\boldsymbol{r}}) \chi_{\frac{1}{2} m_{s}}
$$

where $\ell$ is the orbital angular momentum and $\frac{1}{2}$ is the intrinsic spin of the nucleon, which couple together to give the total spin $j$ (the properties of C-G coefficients are given in [42] and Appendix A.1). The factor $\imath^{\ell}$ has been neglected in Eq. 3.12 because it has no effect on the calculation in this work. For the model of ${ }^{10} \mathrm{~B}$ used, both valence nucleons are in 
the $1 p_{\frac{3}{2}}$ shell so $\ell_{1}=\ell_{2}=1$ and the $\imath^{\ell}$ factors cancel.

The real radial wavefunction in Eq. $3.12, u_{\ell j}(r)$ is normalised to give

$$
\int_{0}^{\infty} r^{2} u_{\ell j}(r)^{2} d r=1
$$

and $Y_{\ell \lambda}(\hat{r})$ is a spherical harmonic as described in Appendix A.4. The spin function, $\chi_{\frac{1}{2} m_{s}}$, describes the intrinsic angular momentum of the valence nucleon and is normalised so that

$$
\left\langle\frac{1}{2} m_{s}^{\prime} \mid \frac{1}{2} m_{s}\right\rangle=\delta_{m_{s}, m_{s}^{\prime}}
$$

For the simple nuclear model of ${ }^{10} \mathrm{~B}$ assumed in this work, the two valence particles are both in the $1 p_{\frac{3}{2}}$ shell and couple together to give the spin $I=3$ for the ground state spin of the nucleus. The nucleon wavefunctions in this case are

$$
\Phi_{1 \frac{3}{2} m}(\boldsymbol{r})=\sum_{\lambda m_{s}}\left(1 \lambda \frac{1}{2} m_{s} \mid \frac{3}{2} m\right) u_{1 \frac{3}{2}}(r) Y_{1 \lambda}(\hat{\boldsymbol{r}}) \chi_{\frac{1}{2} m_{s}}
$$

\subsubsection{Particles and Holes}

As discussed in Section 1.4, the model of ${ }^{10} \mathrm{~B}$ used in this work is a full $1 \mathrm{~s}_{1 / 2}$ shell with a proton and neutron hole in the $1 p_{\frac{3}{2}}$ shell. The relation between an n-hole configuration $j^{2 j+1-n}$ and and n-particle configuration $j^{n}$ is discussed in Chapter 22 of [58]. The relationship between the reduced matrix elements of a tensor operator, $\boldsymbol{T}^{k}(i)$ with $\operatorname{rank} k$ for these two configurations is shown in Eq. 22.43 of [58] to be

$$
\left\langle j^{2 j+1-n} I\left\|\sum_{i=n+1}^{2 j+1} \boldsymbol{T}^{k}(i)\right\| j^{2 j+1-n} I\right\rangle=(-1)^{k+1}\left\langle j^{n} I\left\|\sum_{i=1}^{n} \boldsymbol{T}^{k}(i)\right\| j^{n} I\right\rangle
$$

for $k>0$. For $k=0$

$$
\left\langle j^{2 j+1-n} I\left\|\sum_{i=n+1}^{2 j+1} \boldsymbol{T}^{0}(i)\right\| j^{2 j+1-n} I\right\rangle=\frac{2 j+1-n}{n}\left\langle j^{n} I\left\|\sum_{i=1}^{n} \boldsymbol{T}^{0}(i)\right\| j^{n} I\right\rangle .
$$

Therefore, for the case of this ${ }^{10} \mathrm{~B}$ model, where the two valence-particle description is used, only spin-spin interactions that are even rank in the tensor operator, $\tau_{k_{I} q_{I}}(\boldsymbol{I})$, must have their phase changed for $k_{I}>0$, to convert to a two valence-hole description. 


\subsection{General Folding}

Now that the nuclear wavefunction has been defined, the folding of the effective NN interaction, $V_{N N}(0, i)$, can be written as a modified form of Eq. 3.9

$$
\begin{aligned}
\left(\Psi_{I, M_{f}}^{A}|V| \Psi_{I, M_{i}}^{A}\right) & =\left\langle\Phi_{\text {core }}\left(\boldsymbol{r}_{3} \ldots \boldsymbol{r}_{A}\right)\left|\sum_{i=3}^{A} V_{N N}(0, i)\right| \Phi_{\text {core }}\left(\boldsymbol{r}_{3} \ldots \boldsymbol{r}_{A}\right)\right\rangle \delta_{M_{i}, M_{f}} \\
& +\left\langle\Psi_{I, M_{f}}\left(\boldsymbol{r}_{1}, \boldsymbol{r}_{2}\right)\left|V_{p p}(0,1)+V_{p n}(0,2)\right| \Psi_{I, M_{i}}\left(\boldsymbol{r}_{1}, \boldsymbol{r}_{2}\right)\right\rangle
\end{aligned}
$$

where each part of the second term can also be written in the following way

$$
\int \Psi_{I, M_{f}}^{*}\left(\boldsymbol{r}_{1}, \boldsymbol{r}_{2}\right) V_{N N}(0, i) \Psi_{I, M_{i}}\left(\boldsymbol{r}_{1}, \boldsymbol{r}_{2}\right) d \boldsymbol{r}_{1} d \boldsymbol{r}_{2}
$$

The index $i$ in Eq. 3.19 is, $i=1$ for the proton valence particle $\left(V_{p p}(0,1)\right)$ and $i=2$ for the neutron valence particle $\left(V_{p n}(0,2)\right)$ as labeled in Figure 3.1.

Substituting the coupled nucleon wavefunctions from Eq. 3.11 into Eq. 3.19 gives

$$
\begin{aligned}
\left\langle\Psi_{I, M_{f}}\right. & \left.\left(\boldsymbol{r}_{1}, \boldsymbol{r}_{2}\right)\left|V_{N N}(0, i)\right| \Psi_{I, M_{i}}\left(\boldsymbol{r}_{1}, \boldsymbol{r}_{2}\right)\right\rangle \\
& =\int \sum_{m_{1}^{\prime} m_{2}^{\prime} m_{1} m_{2}} \Phi_{\ell_{1}^{\prime} j_{1}^{\prime} m_{1}^{\prime}}^{*}\left(\boldsymbol{r}_{1}\right)\left(j_{1}^{\prime} m_{1}^{\prime} j_{2}^{\prime} m_{2}^{\prime} \mid I M_{f}\right) \Phi_{\ell_{2}^{\prime} j_{2}^{\prime} m_{2}^{\prime}}^{*}\left(\boldsymbol{r}_{2}\right) V_{N N}(0, i) \\
& \times \Phi_{\ell_{1} j_{1} m_{1}}\left(\boldsymbol{r}_{1}\right)\left(j_{1} m_{1} j_{2} m_{2} \mid I M_{i}\right) \Phi_{\ell_{2} j_{2} m_{2}}\left(\boldsymbol{r}_{2}\right) d \boldsymbol{r}_{1} d \boldsymbol{r}_{2} .
\end{aligned}
$$

We will now choose which valence nucleon will be examined, the proton (1), or the neutron (2). The process for calculating the folded potential is exactly the same for each nucleon (the only difference being which $V_{N N}(0, i)$ is used) but for the sake of this discussion the interacting nucleon will be taken to be the proton, and hence $\left\langle\Phi_{\ell_{2}^{\prime} j_{2}^{\prime} m_{2}^{\prime}}\left(\boldsymbol{r}_{2}\right) \mid \Phi_{\ell_{2} j_{2} m_{2}}\left(\boldsymbol{r}_{2}\right)\right\rangle=$ $\delta_{\ell_{2}^{\prime}, \ell_{2}} \delta_{j_{2}^{\prime}, j_{2}} \delta_{m_{2}^{\prime}, m_{2}}$. Eq. 3.20 can now be rearranged using the relationships between $\mathrm{C}-\mathrm{G}$ coefficients and Racah coefficients, W(abcd,ef), discussed in [42] and Appendix A.2

$$
\begin{aligned}
\left\langle\Psi_{I, M_{f}}\left(\boldsymbol{r}_{1}, \boldsymbol{r}_{2}\right)\left|V_{p p}\left(\boldsymbol{r}_{p}\right)\right| \Psi_{I, M_{i}}\left(\boldsymbol{r}_{1}, \boldsymbol{r}_{2}\right)\right\rangle & \\
= & \int \sum_{m_{1}^{\prime} m_{1} \lambda_{1}^{\prime} m_{s 1}^{\prime} \lambda_{1} m_{s 1}}\left(\ell_{1}^{\prime} \lambda_{1}^{\prime} \frac{1}{2} m_{s 1}^{\prime} \mid j_{1}^{\prime} m_{1}^{\prime}\right) Y_{\ell_{1}^{\prime} \lambda_{1}^{\prime}}^{*}\left(\hat{\boldsymbol{r}}_{1}\right) \chi_{\frac{1}{2} m_{s 1}^{\prime}}^{*} u_{\ell_{1}^{\prime} j_{1}^{\prime}}\left(r_{1}\right) V_{p p}\left(\boldsymbol{r}_{p}\right) \\
& \times\left(\ell_{1} \lambda_{1} \frac{1}{2} m_{s 1} \mid j_{1} m_{1}\right) Y_{\ell_{1} \lambda_{1}}\left(\hat{\boldsymbol{r}}_{1}\right) \chi_{\frac{1}{2} m_{s 1}} u_{\ell_{1} j_{1}}\left(r_{1}\right) d \boldsymbol{r}_{1} \\
& \times(-)^{j_{1}-j_{1}^{\prime}} \frac{\hat{I}}{\hat{j}_{1}^{\prime}} \sum_{k_{I} q_{I}}\left(j_{1} m_{1} k_{I} q_{I} \mid j_{1}^{\prime} m_{1}^{\prime}\right)\left(I M_{i} k_{I} q_{I} \mid I M_{f}\right){\hat{k_{I}}}^{2} W\left(k_{I} j_{1} I j_{2} ; j_{1}^{\prime} I\right),
\end{aligned}
$$

where $\boldsymbol{r}_{p}=\boldsymbol{R}-\boldsymbol{r}_{1}$, as shown in Fig. 3.1 and the nucleon wavefunctions given in Eq. 3.12 have been used. The three red $\mathrm{C}-\mathrm{G}$ coefficients can be rearranged using the relationships 


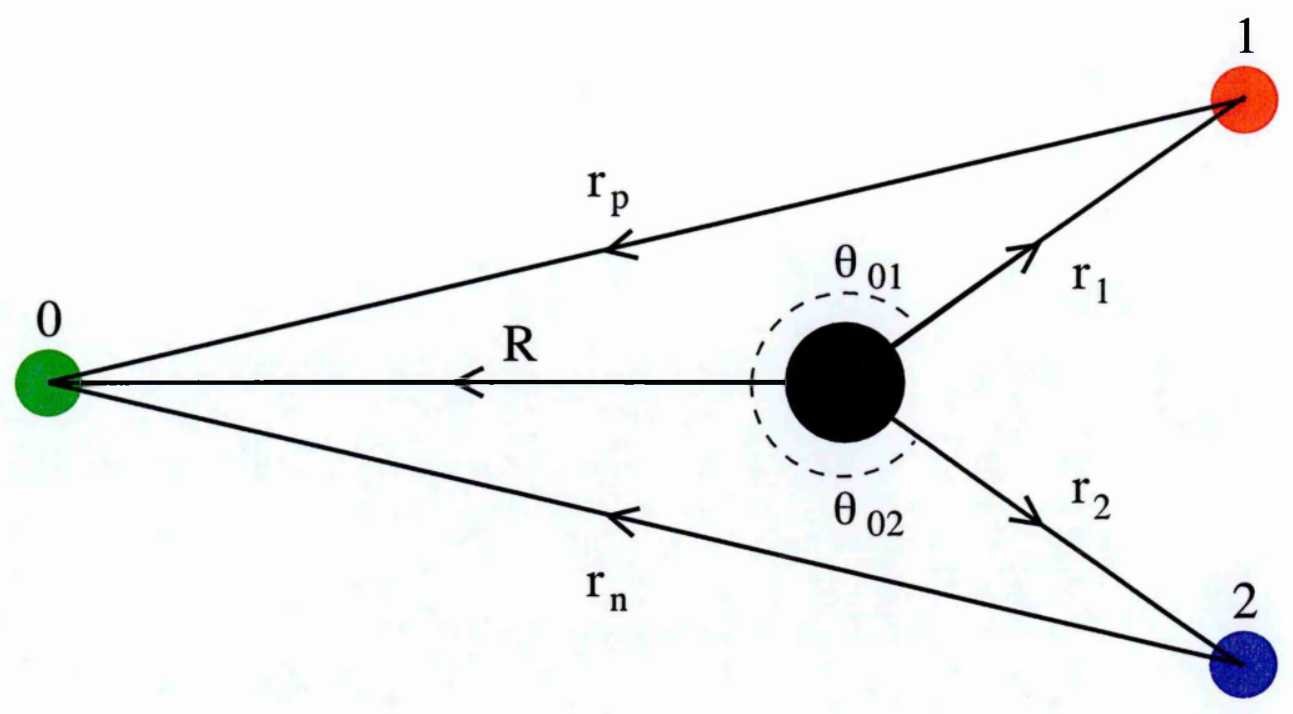

Figure 3.1: The folding model coordinate system, particle 0 is the incident proton, particle 1 is the valence proton and particle 2 is the valence neutron. The projectile-valence nucleon separation is $\boldsymbol{r}_{p}=\boldsymbol{R}-\boldsymbol{r}_{1}$ and $\boldsymbol{r}_{n}=\boldsymbol{R}-\boldsymbol{r}_{2}$ for the proton and neutron respectively.

between C-G coefficients and 9-j symbols discussed in [42] and Appendix A.3

$$
\begin{aligned}
& \sum_{m_{1}^{\prime} m_{1}}\left(\ell_{1}^{\prime} \lambda_{1}^{\prime} \frac{1}{2} m_{s 1}^{\prime} \mid j_{1}^{\prime} m_{1}^{\prime}\right)\left(\ell_{1} \lambda_{1} \frac{1}{2} m_{s 1} \mid j_{1} m_{1}\right)\left(j_{1} m_{1} k_{I} q_{I} \mid j_{1}^{\prime} m_{1}^{\prime}\right) \\
& =\hat{j}_{1} \hat{j}_{1}^{\prime 2} \sum_{k_{1} q_{1} k_{2} q_{2}}\left(\frac{1}{2} m_{s 1} \frac{1}{2}-m_{s 1}^{\prime} \mid k_{1} q_{1}\right)\left(\ell_{1}-\lambda_{1} \ell_{1}^{\prime} \lambda_{1}^{\prime} \mid k_{2} q_{2}\right) \\
& \quad \times\left(k_{1} q_{1} k_{I} q_{I} \mid k_{2} q_{2}\right)(-)^{k_{1}-k_{2}+1 / 2+m_{s 1}^{\prime}+2 j_{1}-\ell_{1}^{\prime}-\lambda_{1}} \hat{k}_{1}\left\{\begin{array}{ccc}
j_{1} & j_{1}^{\prime} & k_{I} \\
\frac{1}{2} & \frac{1}{2} & k_{1} \\
\ell_{1} & \ell_{1}^{\prime} & k_{2}
\end{array}\right\} .
\end{aligned}
$$

Substituting this rearrangement of the C-G coefficients into Eq. 3.21 gives the general folding equation

$$
\begin{aligned}
& \left\langle\Psi_{I, M_{f}}\left(\boldsymbol{r}_{1}, \boldsymbol{r}_{2}\right)\left|V_{p p}\left(\boldsymbol{r}_{p}\right)\right| \Psi_{I, M_{i}}\left(\boldsymbol{r}_{1}, \boldsymbol{r}_{2}\right)\right\rangle \\
& =(-)^{3 j_{1}-j_{1}^{\prime}} \hat{I} \hat{j}_{1} \hat{j}_{1}^{\prime} \int \sum_{\lambda_{1}^{\prime} \lambda_{1} m_{s 1}^{\prime} m_{s 1}} Y_{\ell_{1}^{\prime} \lambda_{1}^{\prime}}^{*}\left(\hat{\boldsymbol{r}}_{1}\right) \chi_{\frac{1}{2} m_{s 1}^{\prime}}^{*} u_{\ell_{1}^{\prime} j_{1}^{\prime}}\left(r_{1}\right) V_{p p}\left(\boldsymbol{r}_{p}\right) Y_{\ell_{1} \lambda_{1}}\left(\hat{\boldsymbol{r}}_{1}\right) \chi_{\frac{1}{2} m_{s 1}} \\
& \times u_{\ell_{1} j_{1}}\left(r_{1}\right) d \boldsymbol{r}_{1}(-)^{\frac{1}{2}+m_{s 1}^{\prime}-\ell_{1}^{\prime}-\lambda_{1}} \sum_{\substack{k_{I} q_{I} \\
k_{1} q_{1} k_{2} q_{2}}} \hat{k}_{I}^{2} W\left(k_{I} j_{1} I j_{2} ; j_{1}^{\prime} I\right)\left(I M_{i} k_{I} q_{I} \mid I M_{f}\right) \\
& \times(-)^{k_{1}-k_{2}} \hat{k}_{1}\left(\frac{1}{2} \cdot m_{s 1} \frac{1}{2}-m_{s 1}^{\prime} \mid k_{1} q_{1}\right)\left(\ell_{1}-\lambda_{1} \ell_{1}^{\prime} \lambda_{1}^{\prime} \mid k_{2} q_{2}\right)\left(k_{1} q_{1} k_{I} q_{I} \mid k_{2} q_{2}\right)\left\{\begin{array}{ccc}
j_{1} & j_{1}^{\prime} & k_{I} \\
\frac{1}{2} & \frac{1}{2} & k_{1} \\
\ell_{1} & \ell_{1}^{\prime} & k_{2}
\end{array}\right\} .
\end{aligned}
$$


This equation can be used to determine the spin-spin potentials for direct and exchange terms in the NN interaction for both the valence proton and neutron particle. For the folding of the specific NN interaction terms in Eq. 3.7, the details of the angular momentum re-coupling methods used to obtain the final folded potentials will be outlined in the following sections and full details given in Appendix C.

\subsubsection{Multipole Expansion Coefficients}

The radial form factor for each term in the NN-interaction shown in Eq. 3.7 can be written using a multipole expansion

$$
v_{p p}\left(\left|\boldsymbol{R}-\boldsymbol{r}_{1}\right|\right)=\sum_{K} f_{K}\left(R, r_{1}\right) P_{K}(\mu),
$$

using the coordinate system shown in Fig. 3.1, this is the potential form factor for the valence proton. The function $f_{K}\left(R, r_{1}\right)$ is given by

$$
f_{K}\left(R, r_{1}\right)=\frac{\hat{K}^{2}}{2} \int_{-1}^{1} P_{K}(\mu) v_{p p}\left(\left|\boldsymbol{R}-\boldsymbol{r}_{1}\right|\right) d \mu
$$

where $P_{K}(\mu)$ is a Legendre Polynomial, $\mu=\cos \theta_{01}$ and $\theta_{01}$ is the angle between $\boldsymbol{R}$ and $\boldsymbol{r}_{1}$ as shown in Fig. 3.1.

\subsection{Central NN Interaction}

Using the multipole expansion in Eq. 3.24 and the following relationship between Legendre Polynomials and spherical harmonics

$$
P_{K}(\mu)=\frac{4 \pi}{\hat{K}^{2}} \sum_{Q} Y_{K Q}(\hat{\boldsymbol{R}}) Y_{K Q}^{*}\left(\hat{\boldsymbol{r}}_{1}\right),
$$

the central term in the NN interaction, $v_{p p}^{\sigma \sigma}\left(r_{p}\right) \sigma_{0} \cdot \sigma_{1}$, can be expressed as

$$
\begin{aligned}
v_{p p}^{\sigma \sigma}\left(r_{p}\right) \boldsymbol{\sigma}_{0} \cdot \boldsymbol{\sigma}_{1} & =4 \pi \sum_{K Q} \frac{f_{K}^{(\sigma \sigma)}\left(R, r_{1}\right)}{\hat{K}^{2}} Y_{K Q}(\hat{\boldsymbol{R}}) Y_{K Q}^{*}\left(\hat{\boldsymbol{r}}_{1}\right) \boldsymbol{\sigma}_{0} \cdot \boldsymbol{\sigma}_{1} \\
& =4 \pi \sum_{K Q} \frac{f_{K}^{(\sigma \sigma)}\left(R, r_{1}\right)}{\hat{K}^{2}} Y_{K Q}(\hat{\boldsymbol{R}}) Y_{K Q}^{*}\left(\hat{\boldsymbol{r}}_{1}\right) \sum_{\mu_{0}}(-)^{\mu_{0}} \sigma_{1 \mu_{0}}^{(0)} \sigma_{1-\mu_{0}}^{(1)} .
\end{aligned}
$$


The folding of this central direct term in the NN interaction using Eq. 3.23 gives the following result

$$
\begin{aligned}
\left\langle\Psi_{I, M_{f}}\left(\boldsymbol{r}_{1}, \boldsymbol{r}_{2}\right)\left|v_{p p}^{\sigma \sigma}\left(r_{p}\right) \boldsymbol{\sigma}_{0} \cdot \boldsymbol{\sigma}_{1}\right| \Psi_{I, M_{i}}\left(\boldsymbol{r}_{1}, \boldsymbol{r}_{2}\right)\right\rangle \\
=(-)^{3 j_{1}-j_{1}^{\prime}} \sqrt{24 \pi} \hat{I} \hat{j}_{1} \hat{j}_{1}^{\prime} \hat{\ell}_{1}^{\prime} \int_{0}^{\infty} r_{1}^{2} d r_{1} u_{\ell_{1}^{\prime} j_{1}^{\prime}}\left(r_{1}\right) \sum_{k_{I} k_{2}} \frac{f_{k_{2}}^{(\sigma \sigma)}\left(R, r_{1}\right)}{\hat{k}_{2}^{2}} u_{\ell_{1} j_{1}}\left(r_{1}\right) \\
\quad \times(-)^{k_{2}}\left(\ell_{1}^{\prime} 0 k_{2} 0 \mid \ell_{1} 0\right) \hat{k_{I}} W\left(k_{I} j_{1} I j_{2} ; j_{1}^{\prime} I\right)\left\{\begin{array}{ccc}
j_{1} & j_{1}^{\prime} & k_{I} \\
\frac{1}{2} & \frac{1}{2} & 1 \\
\ell_{1} & \ell_{1}^{\prime} & k_{2}
\end{array}\right\}\left\langle I, M_{f}\left|\boldsymbol{S}_{k_{I} k_{2}}\right| I, M_{i}\right\rangle .
\end{aligned}
$$

More details of this process are given in Appendix C.1. $\boldsymbol{S}_{k_{I} k_{2}}$ is the generalised spin-spin operator discussed in Section 2.1 and $\left\langle I, M_{f}\left|S_{k_{I} k_{2}}\right| I, M_{i}\right\rangle$ is given by

$$
\left\langle I, M_{f}\left|\boldsymbol{S}_{k_{I} k_{2}}\right| I, M_{i}\right\rangle=\sum_{\mu_{0} q_{I} q_{2}}\left(1 \mu_{0} k_{I} q_{I} \mid k_{2}-q_{2}\right) \tau_{1 \mu_{0}}\left(\boldsymbol{\sigma}_{0}\right)\left\langle I, M_{f}\left|\tau_{k_{I} q_{I}}(\boldsymbol{I})\right| I, M_{i}\right\rangle Y_{k_{2} q_{2}}(\hat{\boldsymbol{R}})(-)^{q_{2}}
$$

\subsubsection{Spin-Spin Potentials for Central Term}

For the model of ${ }^{10} \mathrm{~B}$ used in this work the valence nucleons remain in the $1 p_{\frac{3}{2}}$ shell and therefore have initial and final angular momenta of $\ell_{1}=\ell_{1}^{\prime}=1$. These couple with the intrinsic $\frac{1}{2}$-spin of the nucleon to give total angular momenta of $j_{1}=j_{1}^{\prime}=j_{2}=\frac{3}{2}$ and $I=3$. Inserting these numbers into Eq. 3.29 yields three different types of spin-spin interactions

$$
\begin{aligned}
& U_{10}^{\sigma \sigma}(R)=-2 \sqrt{\frac{\pi}{3}} \int_{0}^{\infty} r_{1}^{2} d r_{1} u_{1 \frac{3}{2}}\left(r_{1}\right)^{2} \int_{-1}^{1} d \mu P_{0}(\mu) v_{p p}^{\sigma \sigma}\left(r_{p}\right) \boldsymbol{S}_{10} \\
& U_{12}^{\sigma \sigma}(\boldsymbol{R})=-\frac{2}{5} \sqrt{\frac{2 \pi}{15}} \int_{0}^{\infty} r_{1}^{2} d r_{1} u_{1 \frac{3}{2}}\left(r_{1}\right)^{2} \int_{-1}^{1} d \mu P_{2}(\mu) v_{p p}^{\sigma \sigma}\left(r_{p}\right) \boldsymbol{S}_{12} \\
& U_{32}^{\sigma \sigma}(\boldsymbol{R})=\frac{3}{5} \sqrt{\frac{2 \pi}{5}} \int_{0}^{\infty} r_{1}^{2} d r_{1} u_{1 \frac{3}{2}}\left(r_{1}\right)^{2} \int_{-1}^{1} d \mu P_{2}(\mu) v_{p p}^{\sigma \sigma}\left(r_{p}\right) \boldsymbol{S}_{32} .
\end{aligned}
$$

These spin-spin potentials are of the form given in Eq. 2.19, $U_{k_{I} k}^{\sigma \sigma}(\boldsymbol{R})=F_{k_{I} k}^{\sigma \sigma}(R) \boldsymbol{S}_{k_{I} k}$, where the superscript ${ }^{\sigma \sigma}$ indicates that these spin-spin interactions come from the folding of the $v_{p p}^{\sigma \sigma}\left(r_{p}\right) \sigma_{0} \cdot \sigma_{1}$ term in the NN interaction

For this model of ${ }^{10} \mathrm{~B}$, the spherical $U_{10}(R)$ and tensor $U_{12}(\boldsymbol{R})$ spin-spin interactions examined in previous work have been obtained from the folding of the $v_{p p}^{\sigma \sigma}\left(r_{p}\right) \sigma_{0} \cdot \sigma_{1}$ term. In addition, a higher rank term $U_{32}(\boldsymbol{R})$, also derived by McAbee [20,21], has been obtained. 


\subsection{Tensor NN Interaction}

Using the multipole expansion in Eq. 3.24, the tensor term in the NN interaction, $v_{p p}^{t r}\left(r_{p}\right) \boldsymbol{S}_{01}$, can be expressed as

$$
\begin{aligned}
v_{p p}^{t r}\left(r_{p}\right) \boldsymbol{S}_{01} & =4 \pi \sum_{K Q} \frac{f_{K}^{(t r)}\left(R, r_{1}\right)}{\hat{K}^{2}} Y_{K Q}(\hat{\boldsymbol{R}}) Y_{K Q}^{*}\left(\hat{\boldsymbol{r}}_{1}\right)\left[3\left(\boldsymbol{\sigma}_{0} \cdot \hat{\boldsymbol{r}}_{p}\right)\left(\boldsymbol{\sigma}_{1} \cdot \hat{\boldsymbol{r}}_{p}\right)-\boldsymbol{\sigma}_{0} \cdot \boldsymbol{\sigma}_{1}\right] \\
& =4 \pi \sum_{K Q} \frac{f_{K}^{(t r)}\left(R, r_{1}\right)}{\hat{K}^{2}} Y_{K Q}(\hat{\boldsymbol{R}}) Y_{K Q}^{*}\left(\hat{\boldsymbol{r}}_{1}\right) 2 \sqrt{\frac{8 \pi}{5}} \sum_{\mu}(-)^{\mu} \tau_{2-\mu}(\boldsymbol{S}) Y_{2 \mu}\left(\hat{\boldsymbol{r}}_{p}\right) .
\end{aligned}
$$

The relationship between these two different forms is derived in Appendix C.2.1. To aid the folding algebra, the second form of the tensor term in which the spin and radial dependence have been separated, Eq. 3.35, will be used. This can be further separated into

$$
\begin{aligned}
\tau_{2-\mu}(\boldsymbol{S}) & =\frac{\sqrt{3}}{2} \sum_{\mu_{1} \mu_{2}}\left(1 \mu_{1} 1 \mu_{2} \mid 2-\mu\right) \tau_{1 \mu_{1}}\left(\boldsymbol{\sigma}_{0}\right) \tau_{1 \mu_{2}}\left(\boldsymbol{\sigma}_{1}\right), \\
r_{p}^{2} Y_{2 \mu}\left(\hat{\boldsymbol{r}}_{p}\right) & =\left|\boldsymbol{R}-\boldsymbol{r}_{1}\right|^{2} Y_{2 \mu}\left(\widehat{\boldsymbol{R}-\boldsymbol{r}_{1}}\right), \\
& =R^{2} Y_{2 \mu}(\hat{\boldsymbol{R}})+r_{1}^{2} Y_{2 \mu}\left(\hat{\boldsymbol{r}}_{1}\right) \\
& -\sqrt{4 \pi} \sqrt{\frac{10}{3}} \sum_{\alpha \beta}(1 \alpha 1 \beta \mid 2 \mu) R Y_{1 \alpha}(\hat{\boldsymbol{R}}) r_{1} Y_{1 \beta}\left(\hat{\boldsymbol{r}}_{1}\right),
\end{aligned}
$$

where Eq. 3.36 separates the spin operator for the total spin $\boldsymbol{S}$ into the spin operators of the component spins, where $\boldsymbol{S}=\frac{1}{2}\left(\sigma_{0}+\sigma_{1}\right)$ as discussed in Appendix C.2.1. Eq. 3.37 is taken from Appendix VI of [42].

\subsubsection{Folding Equation for all Components of the Tensor NN Interaction}

Using the general folding expression, Eq. 3.23, the folding of the tensor term in the NN interaction, $v_{p p}^{t r}\left(r_{p}\right) \boldsymbol{S}_{01}$, given by Eq. 3.35 and separated into the components from Eqs. 
3.36 and 3.37 can be written as

$$
\begin{aligned}
& \left\langle\Psi_{I, M_{f}}\left(\boldsymbol{r}_{1}, \boldsymbol{r}_{2}\right)\left|v_{p p}^{t r}\left(r_{p}\right) \boldsymbol{S}_{01}\right| \Psi_{I, M_{i}}\left(\boldsymbol{r}_{1}, \boldsymbol{r}_{2}\right)\right\rangle \\
& =(-)^{3 j_{1}-j_{1}^{\prime}} \hat{I} \hat{j}_{1} \hat{j}_{1}^{\prime} \int d \boldsymbol{r}_{1} \sum_{\lambda_{1}^{\prime} \lambda_{1} m_{s 1}^{\prime} m_{s 1}} Y_{\ell_{1}^{\prime} \lambda_{1}^{\prime}}^{*}\left(\hat{\boldsymbol{r}}_{1}\right) \chi_{\frac{1}{2} m_{s 1}^{\prime}}^{*} u_{\ell_{1}^{\prime} j_{1}^{\prime}}\left(r_{1}\right) 4 \pi \sum_{K Q} \frac{f_{K}^{(t r)}\left(R, r_{1}\right)}{\hat{K}^{2}} Y_{K Q}(\hat{\boldsymbol{R}}) \\
& \times Y_{K Q}^{*}\left(\hat{\boldsymbol{r}}_{1}\right) 2 \sqrt{\frac{8 \pi}{5}} \sum_{\mu \mu_{0} \mu_{1}}(-)^{\mu} \frac{\sqrt{3}}{2}\left(1 \mu_{0} 1 \mu_{1} \mid 2-\mu\right) \tau_{1 \mu_{0}}\left(\boldsymbol{\sigma}_{0}\right) \tau_{1 \mu_{1}}\left(\boldsymbol{\sigma}_{1}\right) \frac{1}{r_{p}^{2}}\left\{R^{2} Y_{2 \mu}(\hat{\boldsymbol{R}})\right. \\
& \left.+r_{1}^{2} Y_{2 \mu}\left(\hat{\boldsymbol{r}}_{1}\right)-\sqrt{4 \pi} \sqrt{\frac{10}{3}} \sum_{\alpha \beta}(1 \alpha 1 \beta \mid 2 \mu) R Y_{1 \alpha}(\hat{\boldsymbol{R}}) r_{1} Y_{1 \beta}\left(\hat{\boldsymbol{r}}_{1}\right)\right\} Y_{\ell_{1} \lambda_{\mathbf{1}}}\left(\hat{\boldsymbol{r}}_{1}\right) \chi_{\frac{1}{2} m_{\boldsymbol{s} 1}} \\
& \times u_{\ell_{1} j_{1}}\left(r_{1}\right)(-)^{1 / 2+m_{s 1}^{\prime}-\ell_{1}^{\prime}-\lambda_{1}} \sum_{k_{I} q_{I}}{\hat{k_{I}}}^{2} W\left(k_{I} j_{1} I j_{2} ; j_{1}^{\prime} I\right)\left(I M_{i} k_{I} q_{I} \mid I M_{f}\right) \sum_{k_{1} q_{1} k_{2} q_{2}}(-)^{k_{1}-k_{2}} \hat{k}_{1} \\
& \times\left(\frac{1}{2} m_{s 1} \frac{1}{2}-m_{s 1}^{\prime} \mid k_{1} q_{1}\right)\left(\ell_{1}-\lambda_{1} \ell_{1}^{\prime} \lambda_{1}^{\prime} \mid k_{2} q_{2}\right)\left(k_{1} q_{1} k_{I} q_{I} \mid k_{2} q_{2}\right)\left\{\begin{array}{ccc}
j_{1} & j_{1}^{\prime} & k_{I} \\
\frac{1}{2} & \frac{1}{2} & k_{1} \\
\ell_{1} & \ell_{1}^{\prime} & k_{2}
\end{array}\right\} .
\end{aligned}
$$

The substitution of Eq. 3.37 for the spherical harmonic $Y_{2 \mu}\left(\hat{\boldsymbol{r}}_{p}\right)$ has led to three terms highlighted in different colours. The folding of each of these terms will be dealt with separately.

\subsubsection{Spin-Spin Potentials for Tensor Term 1}

Tensor Term 1 will refer only to the first term $R^{2} Y_{2 \mu}(\hat{\boldsymbol{R}})$ in the Eq. 3.38. The folding of this part of the tensor $\mathrm{NN}$ interaction over the valence nucleon wavefunction yields

\section{Term 1}

$$
\begin{aligned}
& =(-)^{3 j_{1}-j_{1}^{\prime}} 6 \sqrt{20 \pi} \hat{I} \hat{j}_{1} \hat{j}_{1}^{\prime} \hat{\ell}_{1}^{\prime} R^{2} \int_{0}^{\infty} r_{1}^{2} d r_{1} u_{\ell_{1}^{\prime} j_{1}^{\prime}}\left(r_{1}\right) \sum_{k_{I} k_{2} C} \frac{f_{k_{2}}^{(t r)}\left(R, r_{1}\right)}{r_{p}^{2} \hat{C}} u_{\ell_{1} j_{1}}\left(r_{1}\right)(-)^{-k_{I}} \\
& \times\left(\ell_{1}^{\prime} 0 k_{2} 0 \mid \ell_{1} 0\right)\left(k_{2} 020 \mid C 0\right) W\left(11 C k_{2} ; 2 k_{I}\right) \hat{k_{I}} W\left(k_{I} j_{1} I j_{2} ; j_{1}^{\prime} I\right)\left\{\begin{array}{ccc}
j_{1} & j_{1}^{\prime} & k_{I} \\
\frac{1}{2} & \frac{1}{2} & 1 \\
\ell_{1} & \ell_{1}^{\prime} & k_{2}
\end{array}\right\}\left\langle I M_{f}\left|\boldsymbol{S}_{k_{I} C}\right| I M_{i}\right\rangle .
\end{aligned}
$$

The full details of the folding method used for this tensor term are given in Appendix C.3.

The individual spin-spin interactions obtained from the folding of this tensor term are obtained by substituting into Eq. 3.39 the values of the angular momenta from the ${ }^{10} \mathrm{~B}$ 
model given in Section 3.5.1 and summing over $k_{2}$

$$
\begin{aligned}
& U_{10}^{t r 1}(R)=\frac{4}{15} \sqrt{3 \pi} R^{2} \int_{0}^{\infty} r_{1}^{2} d r_{1} u_{1 \frac{3}{2}}\left(r_{1}\right)^{2} \int_{-1}^{1} d \mu P_{2}(\mu) \frac{v_{p p}^{t r}\left(r_{p}\right)}{r_{p}^{2}} \boldsymbol{S}_{10} \\
& U_{12}^{t r 1}(\boldsymbol{R})=\frac{2}{3} \sqrt{\frac{6 \pi}{5}} R^{2} \int_{0}^{\infty} r_{1}^{2} d r_{1} u_{1 \frac{3}{2}}\left(r_{1}\right)^{2} \int_{-1}^{1} d \mu\left[P_{0}(\mu)-\frac{P_{2}(\mu)}{5}\right] \frac{v_{p p}^{t r}\left(r_{p}\right)}{r_{p}^{2}} \boldsymbol{S}_{12} \\
& U_{32}^{t r 1}(\boldsymbol{R})=\frac{6}{35} \sqrt{\frac{2 \pi}{5}} R^{2} \int_{0}^{\infty} r_{1}^{2} d r_{1} u_{1 \frac{3}{2}}\left(r_{1}\right)^{2} \int_{-1}^{1} d \mu P_{2}(\mu) \frac{v_{p p}^{t r}\left(r_{p}\right)}{r_{p}^{2}} \boldsymbol{S}_{32} \\
& U_{34}^{\operatorname{tr} 1}(\boldsymbol{R})=-\frac{6}{35} \sqrt{6 \pi} R^{2} \int_{0}^{\infty} r_{1}^{2} d r_{1} u_{1 \frac{3}{2}}\left(r_{1}\right)^{2} \int_{-1}^{1} d \mu P_{2}(\mu) \frac{v_{p p}^{t r}\left(r_{p}\right)}{r_{p}^{2}} \boldsymbol{S}_{34} .
\end{aligned}
$$

These spin-spin interactions are also of the form given in Eq. 2.19, $U_{k_{I} k}^{\operatorname{tr} 1}(\boldsymbol{R})=F_{k_{I} k}^{\operatorname{tr} 1}(R) \boldsymbol{S}_{k_{I} k}$. To denote that these spin-spin potentials come from the folding of tensor Term 1 the superscript ${ }^{t r 1}$ is used. This notation will also be used for the following two tensor terms.

These folded potentials represent just a portion of the contribution of the tensor term in the NN interaction to the spin-spin potentials. In order to determine the whole contribution, the other two terms in Eq. 3.38 must be evaluated.

\subsubsection{Spin-Spin Potentials for Tensor Term 2}

The second term $r_{1}^{2} Y_{2 \mu}\left(\hat{\boldsymbol{r}}_{1}\right)$ from Eq. 3.38 will be referred to as tensor Term 2. The folding of this part of the tensor NN interaction over the valence nucleon wavefunction gives the final result

\section{Term 2}

$$
\begin{aligned}
& =(-)^{3 j_{1}-j_{1}^{\prime}-\ell_{1}^{\prime}+\ell_{1}} 6 \sqrt{20 \pi} \hat{I} \hat{j}_{1} \hat{j}_{1}^{\prime} \hat{\ell}_{1} \hat{\ell}_{1}^{\prime} \int_{0}^{\infty} r_{1}^{4} d r_{1} u_{\ell_{1}^{\prime} j_{1}^{\prime}}\left(r_{1}\right) \sum_{k_{I} k_{2} C K} \frac{f_{K}^{(t r)}\left(R, r_{1}\right)}{r_{p}^{2} \hat{K}^{2}} \\
& \times u_{\ell_{1} j_{1}}\left(r_{1}\right)(-)^{K-k_{I}} \hat{k}_{2}^{2}\left(\ell_{1}^{\prime} 0 K 0 \mid C 0\right)\left(20 \ell_{1} 0 \mid C 0\right) W\left(11 K k_{2} ; 2 k_{I}\right) \\
& \times W\left(2 \ell_{1} K \ell_{1}^{\prime} ; C k_{2}\right) \hat{k_{I}} W\left(k_{I} j_{1} I j_{2} ; j_{1}^{\prime} I\right)\left\{\begin{array}{ccc}
j_{1} & j_{1}^{\prime} & k_{I} \\
\frac{1}{2} & \frac{1}{2} & 1 \\
\ell_{1} & \ell_{1}^{\prime} & k_{2}
\end{array}\right\}\left\langle I M_{f}\left|S_{k_{I} K}\right| I M_{i}\right\rangle,
\end{aligned}
$$

where this folding formalism is described more thoroughly in Appendix C.4.

Substituting the values of the orbital angular momenta and total spins from the ${ }^{10} \mathrm{~B}$ model into Eq. 3.44 and summing over $C$ and $k_{2}$ yields the different spin-spin interactions 
obtained from this term:

$$
\begin{aligned}
U_{10}^{t r 2}(R) & =\frac{4}{15} \sqrt{3 \pi} \int_{0}^{\infty} r_{1}^{4} d r_{1} u_{1 \frac{3}{2}}\left(r_{1}\right)^{2} \int_{-1}^{1} d \mu P_{0}(\mu) \frac{v_{p p}^{t r}\left(r_{p}\right)}{r_{p}^{2}} \boldsymbol{S}_{10} \\
U_{12}^{t r 2}(\boldsymbol{R}) & =\frac{8}{15} \sqrt{\frac{6 \pi}{5}} \int_{0}^{\infty} r_{1}^{4} d r_{1} u_{1 \frac{3}{2}}\left(r_{1}\right)^{2} \int_{-1}^{1} d \mu P_{2}(\mu) \frac{v_{p p}^{t r}\left(r_{p}\right)}{r_{p}^{2}} \boldsymbol{S}_{12} \\
U_{32}^{t r 2}(\boldsymbol{R}) & =\frac{6}{35} \sqrt{\frac{2 \pi}{5}} \int_{0}^{\infty} r_{1}^{4} d r_{1} u_{1 \frac{3}{2}}\left(r_{1}\right)^{2} \int_{-1}^{1} d \mu P_{2}(\mu) \frac{v_{p p}^{t r}\left(r_{p}\right)}{r_{p}^{2}} \boldsymbol{S}_{32} \\
U_{34}^{t r 2}(\boldsymbol{R}) & =-\frac{6}{35} \sqrt{6 \pi} \int_{0}^{\infty} r_{1}^{4} d r_{1} u_{1 \frac{3}{2}}\left(r_{1}\right)^{2} \int_{-1}^{1} d \mu P_{4}(\mu) \frac{v_{p p}^{t r}\left(r_{p}\right)}{r_{p}^{2}} \boldsymbol{S}_{34} .
\end{aligned}
$$

\subsubsection{Spin-Spin Potentials for Tensor Term 3}

The last term to be examined in 3.38 will be referred to as tensor Term 3. The folding of this part of the tensor NN interaction over the valence nucleon wavefunction gives the result

\section{Term 3}

$$
\begin{aligned}
& =(-)^{3 j_{1}-j_{1}^{\prime}-\ell_{1}^{\prime}+\ell_{1}} 30 \sqrt{6} \sqrt{4 \pi} \hat{I} \hat{j}_{1} \hat{j}_{1}^{\prime} \hat{\ell}_{1} \hat{\ell}_{1}^{\prime} R \int_{0}^{\infty} r_{1}^{3} d r_{1} u_{\ell_{1}^{\prime} j_{1}^{\prime}}\left(r_{1}\right) \sum_{k_{I} k_{2} C K C^{\prime}} \frac{f_{K}^{(t r)}\left(R, r_{1}\right)}{r_{p}^{2} \hat{C}^{\prime}} u_{\ell_{1} j_{1}}\left(r_{1}\right) \\
& \times(-)^{K-k_{I}} \hat{k}_{2}^{2}\left(\ell_{1}^{\prime} 0 K 0 \mid C 0\right)\left(10 \ell_{1} 0 \mid C 0\right)\left(K 010 \mid C^{\prime} 0\right) W\left(11 C^{\prime} k_{2} ; 2 k_{I}\right) W\left(1 \ell_{1} K \ell_{1}^{\prime} ; C k_{2}\right) \\
& \times W\left(k_{2} 1 C^{\prime} 1 ; K 2\right) \hat{k}_{I} W\left(k_{I} j_{1} I j_{2} ; j_{1}^{\prime} I\right)\left\{\begin{array}{ccc}
j_{1} & j_{1}^{\prime} & k_{I} \\
\frac{1}{2} & \frac{1}{2} & 1 \\
\ell_{1} & \ell_{1}^{\prime} & k_{2}
\end{array}\right\}\left\langle I M_{f}\left|S_{k_{I} C^{\prime}}\right| I M_{i}\right\rangle,
\end{aligned}
$$

where this folding is worked through in Appendix C.5.

Substituting the values of the angular momenta from the $1 p_{\frac{3}{2}}$ valence nucleons in the ${ }^{10} \mathrm{~B}$ model into Eq. 3.49 and summing over $K, C$ and $k_{2}$ yields the spin-spin interactions derived from this part of the tensor $\mathrm{NN}$ interaction

$$
\begin{aligned}
& U_{10}^{t r 3}(R)=-\frac{8}{15} \sqrt{3 \pi} R \int_{0}^{\infty} r_{1}^{3} d r_{1} u_{1 \frac{3}{2}}\left(r_{1}\right)^{2} \int_{-1}^{1} d \mu P_{1}(\mu) \frac{v_{p p}^{t r}\left(r_{p}\right)}{r_{p}^{2}} \boldsymbol{S}_{10} \\
& U_{12}^{t r 3}(\boldsymbol{R})=-\frac{2}{25} \sqrt{\frac{6 \pi}{5}} R \int_{0}^{\infty} r_{1}^{3} d r_{1} u_{1 \frac{3}{2}}\left(r_{1}\right)^{2} \int_{-1}^{1} d \mu\left[\frac{43}{3} P_{1}(\mu)-P_{3}(\mu)\right] \frac{v_{p p}^{t r}\left(r_{p}\right)}{r_{p}^{2}} \boldsymbol{S}_{12} \\
& U_{32}^{t r 3}(\boldsymbol{R})=-\frac{6}{25} \sqrt{\frac{2 \pi}{5}} R \int_{0}^{\infty} r_{1}^{3} d r_{1} u_{1 \frac{3}{2}}\left(r_{1}\right)^{2} \int_{-1}^{1} d \mu\left[P_{1}(\mu)-\frac{3}{7} P_{3}(\mu)\right] \frac{v_{p p}^{t r}\left(r_{p}\right)}{r_{p}^{2}} \boldsymbol{S}_{32} \\
& U_{34}^{t r 3}(\boldsymbol{R})=\frac{12}{35} \sqrt{6 \pi} R \int_{0}^{\infty} r_{1}^{3} d r_{1} u_{1 \frac{3}{2}}\left(r_{1}\right)^{2} \int_{-1}^{1} d \mu P_{3}(\mu) \frac{v_{p p}^{t r}\left(r_{p}\right)}{r_{p}^{2}} \boldsymbol{S}_{34} .
\end{aligned}
$$




\subsubsection{Spin-Spin Potentials for All Tensor Terms}

Summing the potentials from all three tensor terms yields the spin-spin interactions from the folding of the whole tensor term in the NN interaction for protons elastically scattering from ${ }^{10} \mathrm{~B}$

$$
\begin{aligned}
U_{10}^{t r}(R) & =\frac{4}{15} \sqrt{3 \pi} \int_{0}^{\infty} r_{1}^{2} d r_{1} u_{1 \frac{3}{2}}\left(r_{1}\right)^{2} \int_{-1}^{1} d \mu\left[r_{1}^{2} P_{0}(\mu)+R^{2} P_{2}(\mu)\right. \\
& \left.-2 R r_{1} P_{1}(\mu)\right] \frac{v_{p p}^{t r}\left(r_{p}\right)}{r_{p}^{2}} S_{10} \\
U_{12}^{t r}(\boldsymbol{R}) & =\frac{2}{15} \sqrt{\frac{6 \pi}{5}} \int_{0}^{\infty} r_{1}^{2} d r_{1} u_{1 \frac{3}{2}}\left(r_{1}\right)^{2} \int_{-1}^{1} d \mu\left[5 R^{2} P_{0}(\mu)+\left(4 r_{1}^{2}-R^{2}\right) P_{2}(\mu)\right. \\
& \left.-\frac{43}{5} R r_{1} P_{1}(\mu)+\frac{3}{5} R r_{1} P_{3}(\mu)\right] \frac{v_{p p}^{t r}\left(r_{p}\right)}{r_{p}^{2}} \boldsymbol{S}_{12}, \\
U_{32}^{t r}(\boldsymbol{R}) & =\frac{6}{35} \sqrt{\frac{2 \pi}{5}} \int_{0}^{\infty} r_{1}^{2} d r_{1} u_{1 \frac{3}{2}}\left(r_{1}\right)^{2} \int_{-1}^{1} d \mu\left[\left(r_{1}^{2}+R^{2}\right) P_{2}(\mu)-\frac{7}{5} R r_{1} P_{1}(\mu)\right. \\
& \left.-\frac{3}{5} R r_{1} P_{3}(\mu)\right] \frac{v_{p p}^{t r}\left(r_{p}\right)}{r_{p}^{2}} \boldsymbol{S}_{32}, \\
U_{34}^{t r}(\boldsymbol{R}) & =-\frac{6}{35} \sqrt{6 \pi} \int_{0}^{\infty} r_{1}^{2} d r_{1} u_{1 \frac{3}{2}}\left(r_{1}\right)^{2} \int_{-1}^{1} d \mu\left[R^{2} P_{2}(\mu)+r_{1}^{2} P_{4}(\mu)\right. \\
& \left.-2 R r_{1} P_{3}(\mu)\right] \frac{v_{p p}^{t r}\left(r_{p}\right)}{r_{p}^{2}} \boldsymbol{S}_{34} .
\end{aligned}
$$

The folding of the tensor $v_{p p}^{t r}\left(r_{p}\right) \boldsymbol{S}_{01}$ term in the NN interaction also yields the spherical $U_{10}(R)$ and tensor $U_{12}(\boldsymbol{R})$ spin-spin interactions examined in previous work (see Section 1.2). Also obtained is the higher rank term, $U_{32}(\boldsymbol{R})$, from McAbee [20,21]. A new spinspin interaction $U_{34}(\boldsymbol{R})$, which goes beyond the work of McAbee, is also derived for the first time.

Combining the spin-spin potentials from the three tensor terms in Eqs. $3.54-3.54$ enables a simple check of the folding of the direct tensor NN interaction term to be performed. When $\boldsymbol{r}_{p}=0$ (i.e. $\boldsymbol{R}=\boldsymbol{r}_{1}$ and $\mu=1$ ) the factor $1 / r_{p}^{2} \rightarrow \infty$ in the equations above. For these potentials to remain physical for all values of $\boldsymbol{r}_{p}$, the terms in the square brackets must be zero when $\boldsymbol{r}_{p}=0$. Expressing the Legendre Polynomials in the equations above in terms of $\mu$ shows that the terms in the square brackets do indeed sum to zero for $r_{p}=0$. 


\subsection{Analytic $\mu$ Integration}

The form factors of the different components of the direct NN interaction given in Eq. 3.7 are chosen to be Yukawa functions (see Section 4.3). This enables the $\mu$ integrals of the folded potentials to be performed analytically. This is done using the relation [49]

$$
\int_{-1}^{1} d \mu P_{K}(\mu) \sum_{j=1}^{N} V_{j} \frac{e^{-\left(\left|\boldsymbol{R}-\boldsymbol{r}_{1}\right| / R_{j}\right)}}{\left|\boldsymbol{R}-\boldsymbol{r}_{1}\right| / R_{j}}=-2 \sum_{j=1}^{N} V_{j} j_{K}\left(i r_{<} / R_{j}\right) h_{K}^{(1)}\left(i r_{>} / R_{j}\right)
$$

where the $V_{j}$ 's are complex strengths in $\mathrm{MeV}\left(\mathrm{MeV}_{\mathrm{fm}}{ }^{-2}\right.$ for the tensor terms) of the Yukawa functions and the $R_{j}$ 's are the range parameters. $j_{K}(x)$ is a spherical Bessel function of the first kind and $h_{K}^{(1)}(x)$ is a spherical Bessel function of the third kind [49]. The arguments of these two Bessel functions depend on whether $R$ or $r_{1}$ is greater. When $R>r_{1}, r_{>}=R$ and $r_{<}=r_{1}$ and visa-versa for $R<r_{1}$.

\subsection{Spin-Orbit Terms}

While this work focuses on spin-spin interactions from the folding of the $\sigma_{0} \cdot \sigma_{1}$ and tensor terms in the NN interaction, spin-spin potentials derived from the folding of the spin-orbit term were also proposed by Feshbach [1]. Some studies of the first order $\boldsymbol{I} \cdot \boldsymbol{\ell}$ term have been performed, where $\ell$ is the relative orbital angular momentum of the projectile and target [56].

Studies of this spin-orbit interaction have mainly focused on projectiles with zero spin as the $\boldsymbol{I} \cdot \boldsymbol{\ell}$ term arises even if the projectile spin is zero. The effect of this interaction has been studied in elastic ${ }^{4} \mathrm{He}$ - and ${ }^{3} \mathrm{He}$-nucleus scattering [59-61]. These works obtained phenomenological strengths of the $\boldsymbol{I} \cdot \boldsymbol{\ell}$ potential which were of the order of $1 \mathrm{MeV} . \mathrm{A}$ theoretical description of these works was attempted by Rawitscher [62] who considered $\alpha$-nucleus scattering and folded a phenomenological $\alpha+\mathrm{N}$ potential over the nucleons in the target nucleus. Rawitscher found the strengths of these potentials to be an order of magnitude below the phenomenological values.

Microscopic studies of the $\boldsymbol{I} \cdot \boldsymbol{\ell}$ term have also been carried out $[63,64]$. Both these works obtain the $\boldsymbol{I} \cdot \boldsymbol{\ell}$ potential by folding over the NN spin-orbit force including a single nucleon exchange term (see Chapter 5 for a discussion of exchange). Love [63] calculated the strength of this interaction for the scattering of ${ }^{4} \mathrm{He}$ from ${ }^{9} \mathrm{Be}$ and ${ }^{59} \mathrm{Co}$ and found the contribution from the exchange term to be much more important that the direct term. Petrovich et al. [64] used a folding model to calculate the strength of the $\boldsymbol{I} \cdot \boldsymbol{\ell}$ term for heavy-ion elastic scattering. Both works obtained theoretical spin-spin potentials that were fairly weak [64]. 
In the search for work on the target spin dependent $\boldsymbol{I} \cdot \boldsymbol{\ell}$ interaction none were found detailing the effect of this term on $D_{N N}$. It is unclear if a term with a dependence on the target spin but no dependence on the projectile spin would cause a significant deviation of $D_{N N}$ from unity. The folding of the spin-orbit term in the NN interaction would lead to higher order spin-spin interactions which do depend on the projectile spin. Their effect on $D_{N N}$ will be the subject of future work.

\subsection{Folding Checks}

The volume integrals of the radial form factors of the spin-spin interactions can be used as a check of the folding model. For the valence proton, ignoring the spin dependence for now, the folded potentials are of the form

$$
\left\langle I M_{f}|U(R)| I M_{i}\right\rangle=U_{M_{f} M_{i}}(R)=\iint d \boldsymbol{r}_{1} d \boldsymbol{r}_{2} \Psi_{I, M_{f}}^{*}\left(\boldsymbol{r}_{1}, \boldsymbol{r}_{2}\right) v_{p p}\left(\left|\boldsymbol{R}-\boldsymbol{r}_{1}\right|\right) \Psi_{I, M_{i}}\left(\boldsymbol{r}_{1}, \boldsymbol{r}_{2}\right) .
$$

Integrating over all space on both sides and using the substitution $\boldsymbol{x}=\boldsymbol{R}-\boldsymbol{r}_{1}$ leads to

$$
4 \pi \int_{0}^{\infty} R^{2} d R U_{M_{f} M_{i}}(R)=4 \pi \int_{0}^{\infty} x^{2} d x v_{p p}(x) \iint d \boldsymbol{r}_{1} d \boldsymbol{r}_{2} \Psi_{I, M_{f}}^{*}\left(\boldsymbol{r}_{1}, \boldsymbol{r}_{2}\right) \Psi_{I, M_{i}}\left(\boldsymbol{r}_{1}, \boldsymbol{r}_{2}\right) .
$$

Using the Yukawa form (see Section 4.3, Eq. 4.10), for the radial form factors of the terms in the NN interaction gives

$$
\int_{0}^{\infty} R^{2} d R U_{M_{f} M_{i}}(R)=\delta_{M_{f}, M_{1}} \int_{0}^{\infty} x^{2} d x \sum_{j=1}^{N} V_{j} R_{j} \frac{\exp \left(-x / R_{j}\right)}{x}=\delta_{M_{f}, M_{1}} \sum_{j=1}^{N} V_{j}\left(R_{j}\right)^{3} .
$$

Use of the Yukawa functions enables the volume integrals of the NN interaction terms to be solved analytically giving a simple expression for the radial integrals of the folded potentials. The spin dependence of the terms in the NN interaction used to derive the folded spin-spin potentials means Eq. 3.61 has to be slightly modified to allow for spin operators between the initial and final valence nucleon wavefunction in Eq. 3.60. This will be discussed in the next two sections.

\subsubsection{Checks of Folded Potentials from Central NN Term}

The folded potentials calculated from this term in the NN interaction are denoted by $U_{k_{I} k}^{\sigma \sigma}(\boldsymbol{R})=F_{k_{I} k}^{\sigma \sigma}(R) \boldsymbol{S}_{k_{I} k}$. For $k_{I}=1$, and $k=0$, the spin-spin operator is independent of 
$R$ and Eq. 3.60 becomes

$$
\begin{aligned}
\int_{0}^{\infty} R^{2} d R F_{10}^{\sigma \sigma}(R)\left\langle I M_{f}\left|\boldsymbol{S}_{10}\right| I M_{i}\right\rangle & =\int_{0}^{\infty} x^{2} d x v_{p p}^{\sigma \sigma}(x) \\
& \times \iint d \boldsymbol{r}_{1} d \boldsymbol{r}_{2} \Psi_{I, M_{f}}^{*}\left(\boldsymbol{r}_{1}, \boldsymbol{r}_{2}\right) \boldsymbol{\sigma}_{0} \cdot \boldsymbol{\sigma}_{1} \Psi_{I, M_{i}}\left(\boldsymbol{r}_{1}, \boldsymbol{r}_{2}\right), \\
& =\int_{0}^{\infty} x^{2} d x v_{p p}^{\sigma \sigma}(x) \times A^{\sigma \sigma}\left\langle I M_{f}\left|\boldsymbol{S}_{10}\right| I M_{i}\right\rangle, \\
\Rightarrow \int_{0}^{\infty} R^{2} d R F_{10}^{\sigma \sigma}(R) & =\int_{0}^{\infty} x^{2} d x v_{p p}^{\sigma \sigma}(x) \times A^{\sigma \sigma}=\sum_{j=1}^{N} V_{j}^{C E}\left(R_{j}^{C E}\right)^{3} \times A^{\sigma \sigma}
\end{aligned}
$$

For the valence nucleon wavefunction used in this work (see Section 3.3.1), the coefficient $A^{\sigma \sigma}$ can be found using standard recoupling techniques in a process analogous to the folding of the whole $v_{p p}^{\sigma \sigma}\left(\boldsymbol{r}_{p}\right) \sigma_{0} \cdot \sigma_{1}$ term (detailed in Appendix C.1). This gives $A^{\sigma \sigma}$ as

$$
A^{\sigma \sigma}=-\sqrt{4 \pi} \sqrt{6} \hat{j}_{1}^{\prime} \hat{j}_{1} \hat{I}(-)^{\ell_{1}+1 / 2-j_{1}^{\prime}} W\left(j_{1} \frac{1}{2} j_{1}^{\prime} \frac{1}{2} ; \ell_{1} 1\right) W\left(1 j_{1} I j_{2} ; j_{1}^{\prime} I\right)
$$

For $k_{I}=1$ or 3 and $k=2$, this check has to be modified slightly because the operators $\boldsymbol{S}_{12}$ and $\boldsymbol{S}_{32}$ both contain $Y_{2 q}(\hat{\boldsymbol{R}})$. The volume integrals of these folded potentials are

$$
\int d \boldsymbol{R} F_{12}^{\sigma \sigma}(R) \boldsymbol{S}_{12}=\int d \boldsymbol{R} F_{32}^{\sigma \sigma}(R) \boldsymbol{S}_{32}=0
$$

because the integration over all angles of a spherical harmonics of rank 2 is zero. In this case, to get a non zero result, the folded potential is multiplied by $R^{2} Y_{2 q^{\prime}}^{*}(\hat{\boldsymbol{R}})$ then integrated over all space

$$
\begin{aligned}
\int d \boldsymbol{R} R^{2} Y_{2 q^{\prime}}^{*}(\hat{\boldsymbol{R}}) F_{k_{I} 2}^{\sigma \sigma}(R)\left\langle I M_{f}\left|\boldsymbol{S}_{k_{I} 2}\right| I M_{i}\right\rangle & =\int d \boldsymbol{x} v_{p p}^{\sigma \sigma}(x) \iint d \boldsymbol{r}_{1} d \boldsymbol{r}_{2} \Psi_{I, M_{f}}^{*}\left(\boldsymbol{r}_{1}, \boldsymbol{r}_{2}\right) \\
& \times\left(\left|\boldsymbol{x}+\boldsymbol{r}_{1}\right|\right)^{2} Y_{2 q^{\prime}}^{*}\left(\boldsymbol{x} \hat{+} \boldsymbol{r}_{1}\right) \boldsymbol{\sigma}_{0} \cdot \boldsymbol{\sigma}_{1} \Psi_{I, M_{i}}\left(\boldsymbol{r}_{1}, \boldsymbol{r}_{2}\right)
\end{aligned}
$$

where $\boldsymbol{R}=\boldsymbol{x}+\boldsymbol{r}_{1}$. Using Eq. 3.37,

$$
\begin{aligned}
\left(\left|\boldsymbol{x}+\boldsymbol{r}_{1}\right|\right)^{2} Y_{2 q^{\prime}}^{*}\left(\boldsymbol{x} \hat{+} \boldsymbol{r}_{1}\right) & =x^{2} Y_{2 q^{\prime}}^{*}(\hat{\boldsymbol{x}})+r_{1}^{2} Y_{2 q^{\prime}}^{*}\left(\hat{\boldsymbol{r}}_{1}\right) \\
& +\sqrt{4 \pi} \sqrt{\frac{10}{3}} \sum_{\alpha \beta}\left(1 \alpha 1 \beta \mid 2 q^{\prime}\right) x Y_{1 \alpha}^{*}(\hat{\boldsymbol{x}}) r_{1} Y_{1 \beta}^{*}\left(\hat{\boldsymbol{r}}_{1}\right)
\end{aligned}
$$

Only the second term, $r_{1}^{2} Y_{2 q^{\prime}}^{*}\left(\hat{\boldsymbol{r}}_{1}\right)$, is non zero when substituted into the right hand side of Eq. 3.65 and integrated over all angles. Selecting only this term means Eq. 3.65 can 
now be written as

$$
\begin{gathered}
\int d \boldsymbol{R} R^{2} Y_{2 q^{\prime}}^{*}(\hat{\boldsymbol{R}}) F_{k_{I} 2}^{\sigma \sigma}(R) \sum_{\mu_{0} q_{I} q}\left(1 \mu_{0} k_{I} q_{I} \mid 2-q\right) \tau_{1 \mu_{0}}\left(\boldsymbol{\sigma}_{0}\right)\left\langle I M_{f}\left|\tau_{k_{I} q_{I}}(\boldsymbol{I})\right| I M_{i}\right\rangle Y_{2 q}(\hat{\boldsymbol{R}})(-)^{q} \\
=\int d \boldsymbol{x} v_{p p}^{\sigma \sigma}(x) \iint d \boldsymbol{r}_{1} d \boldsymbol{r}_{2} \Psi_{I, M_{f}}^{*}\left(\boldsymbol{r}_{1}, \boldsymbol{r}_{2}\right) r_{1}^{2} Y_{2 q^{\prime}}^{*}\left(\hat{\boldsymbol{r}}_{1}\right) \boldsymbol{\sigma}_{0} \cdot \boldsymbol{\sigma}_{1} \Psi_{I, M_{i}}\left(\boldsymbol{r}_{1}, \boldsymbol{r}_{2}\right)
\end{gathered}
$$

where Eq. 3.30 has been used for $\left\langle I M_{f}\left|S_{k_{I} 2}\right| I M_{i}\right\rangle$. Integrating over all angles $d \Omega_{R}$ and $d \Omega_{x}$ gives

$$
\begin{aligned}
& \int_{0}^{\infty} R^{4} d R F_{k_{I} 2}^{\sigma \sigma}(R) \sum_{\mu_{0} q_{I}}\left(1 \mu_{0} k_{I} q_{I} \mid 2-q\right) \tau_{1 \mu_{0}}\left(\boldsymbol{\sigma}_{0}\right)\left\langle I M_{f}\left|\tau_{k_{I} q_{I}}(\boldsymbol{I})\right| I M_{i}\right\rangle(-)^{q} \\
& =4 \pi \int_{0}^{\infty} x^{2} d x v_{p p}^{\sigma \sigma}(x) \iint d \boldsymbol{r}_{1} d \boldsymbol{r}_{2} \Psi_{I, M_{f}}^{*}\left(\boldsymbol{r}_{1}, \boldsymbol{r}_{2}\right) r_{1}^{2} Y_{2 q^{\prime}}^{*}\left(\hat{\boldsymbol{r}}_{1}\right) \boldsymbol{\sigma}_{0} \cdot \boldsymbol{\sigma}_{1} \Psi_{I, M_{i}}\left(\boldsymbol{r}_{1}, \boldsymbol{r}_{2}\right), \\
& =4 \pi \int_{0}^{\infty} x^{2} d x v_{p p}^{\sigma \sigma}(x) \times B_{k_{I}}^{\sigma \sigma} \sum_{\mu_{0} q_{I}}\left(1 \mu_{0} k_{I} q_{I} \mid 2-q\right) \tau_{1 \mu_{0}}\left(\boldsymbol{\sigma}_{0}\right)\left\langle I M_{f}\left|\tau_{k_{I} q_{I}}(\boldsymbol{I})\right| I M_{i}\right\rangle(-)^{q} \\
& \Rightarrow \int R^{4} d R F_{k_{I} 2}^{\sigma \sigma}(R)=4 \pi \int_{0}^{\infty} x^{2} d x v_{p p}^{\sigma \sigma}(x) \times B_{k_{I}}^{\sigma \sigma}=4 \pi \sum_{j=1}^{N} V_{j}^{C E}\left(R_{j}^{C E}\right)^{3} \times B_{k_{I}}^{\sigma \sigma},
\end{aligned}
$$

The coefficient $B_{k_{I}}^{\sigma \sigma}$ can be found using the valence nucleon wavefunctions described in Section 3.3.1 and the recoupling techniques detailed in Appendix C

$$
B_{k_{I}}^{\sigma \sigma}=\frac{-\sqrt{6}}{\sqrt{4 \pi}} \hat{\ell}_{1}^{\prime} \hat{j}_{1}^{\prime} \hat{j_{1}} \hat{I} \hat{k}_{I}\left(\ell_{1}^{\prime} 020 \mid \ell_{1} 0\right) W\left(k_{I} j_{1}^{\prime} I j_{2} ; j_{1} I\right)\left\{\begin{array}{ccc}
2 & 1 & k_{I} \\
\ell_{1}^{\prime} & \frac{1}{2} & j_{1}^{\prime} \\
\ell_{1} & \frac{1}{2} & j_{1}
\end{array}\right\} \int_{0}^{\infty} r_{1}^{4} d r_{1} u_{\ell_{1}^{\prime} j_{1}^{\prime}}\left(r_{1}\right) u_{\ell_{1} j_{1}}\left(r_{1}\right)
$$

for the model of ${ }^{10} \mathrm{~B}$ used in this work, the only values of $k_{I}$ which lead to a non-zero value for $B_{k_{I}}^{\sigma \sigma}$ are $k_{I}=1$ and 3 .

\subsubsection{Checks of Folded Potentials from Tensor NN Term}

The folding of the tensor term in the NN interactions also yields spin-spin potentials of the form $U_{k_{I} k}^{t r}(\boldsymbol{R})=F_{k_{I} k}^{t r}(R) \boldsymbol{S}_{k_{I} k}$. For $k_{I}=1 . k=0$ the properties of rank 2 spherical harmonics discussed in the previous section mean that

$$
\begin{aligned}
\int d \boldsymbol{R} F_{10}^{t r}(R)\left\langle I M_{f}\left|\boldsymbol{S}_{10}\right| I M_{i}\right\rangle=0 & =2 \sqrt{\frac{8 \pi}{5}} \int d \boldsymbol{x} v_{p p}^{t r}(x) \sum_{\mu} Y_{2 \mu}(\hat{\boldsymbol{x}}) \iint d \boldsymbol{r}_{1} d \boldsymbol{r}_{2} \\
& \times \Psi_{I, M_{f}}^{*}\left(\boldsymbol{r}_{1}, \boldsymbol{r}_{2}\right)(-)^{\mu} \tau_{2-\mu}(\boldsymbol{S}) \Psi_{I, M_{i}}\left(\boldsymbol{r}_{1}, \boldsymbol{r}_{2}\right)
\end{aligned}
$$


where the form of the tensor NN interaction given in Eq. 3.35 has been used.

For $k_{I}=1$ or 3 and $k=2$, we can use the same folding check method for the corresponding spin-spin potential terms derived from the folding of the central $v_{p p}^{\sigma \sigma}\left(r_{p}\right) \sigma_{0}$. $\sigma_{1}$ NN interaction term

$$
\begin{aligned}
& \int d \boldsymbol{R} R^{2} Y_{2 q^{\prime}}^{*}(\hat{\boldsymbol{R}}) F_{k_{I} 2}^{t r}(R)\left\langle I M_{f}\left|\boldsymbol{S}_{k_{I}}\right| I M_{i}\right\rangle=2 \sqrt{\frac{8 \pi}{5}} \int d \boldsymbol{x} v_{p p}^{t r}(x) \sum_{\mu} Y_{2 \mu}(\hat{\boldsymbol{x}}) \\
& \quad \times \iint d \boldsymbol{r}_{1} d \boldsymbol{r}_{2} \Psi_{I, M_{f}}^{*}\left(\boldsymbol{r}_{1}, \boldsymbol{r}_{2}\right)\left(\left|\boldsymbol{x}+\boldsymbol{r}_{1}\right|\right)^{2} Y_{2 q^{\prime}}^{*}\left(\boldsymbol{x} \hat{+} \boldsymbol{r}_{1}\right)(-)^{\mu} \tau_{2-\mu}(\boldsymbol{S}) \Psi_{I, M_{i}}\left(\boldsymbol{r}_{1}, \boldsymbol{r}_{2}\right)
\end{aligned}
$$

which is analogous to Eq. 3.65. This time, of the three terms in Eq. 3.66, the only one which does not integrate to zero when inserted into the right hand side of Eq. 3.71 is $x^{2} Y_{2 q^{\prime}}^{*}(\hat{\boldsymbol{x}})$. Therefore

$$
\begin{aligned}
\int d \boldsymbol{R} R^{2} Y_{2 q^{\prime}}^{*}(\hat{\boldsymbol{R}}) F_{k_{I} 2}^{t r}(R) \sum_{\mu_{0} q_{I} q}\left(1 \mu_{0} k_{I} q_{I} \mid 2-q\right) \tau_{1 \mu_{0}}\left(\boldsymbol{\sigma}_{0}\right)\left\langle I M_{f}\left|\tau_{k_{I} q_{I}}(\boldsymbol{I})\right| I M_{i}\right\rangle Y_{2 q}(\hat{\boldsymbol{R}})(-)^{q} \\
=2 \sqrt{\frac{8 \pi}{5}} \int d \boldsymbol{x} x^{2} Y_{2 q^{\prime}}^{*}(\hat{\boldsymbol{x}}) v_{p p}^{t r}(x) \sum_{\mu} Y_{2 \mu}(\hat{\boldsymbol{x}}) \\
\times \iint d \boldsymbol{r}_{1} d \boldsymbol{r}_{2} \Psi_{I, M_{f}}^{*}\left(\boldsymbol{r}_{1}, \boldsymbol{r}_{2}\right)(-)^{\mu} \tau_{2-\mu}(\boldsymbol{S}) \Psi_{I, M_{i}}\left(\boldsymbol{r}_{1}, \boldsymbol{r}_{2}\right)
\end{aligned}
$$

where the form of $\left\langle I M_{f}\left|S_{k_{I}}\right| I M_{i}\right\rangle$ has been included using Eq. 3.30. Now, integrating over all angles $d \Omega_{R}$ and $d \Omega_{x}$ leads to

$$
\begin{gathered}
\int_{0}^{\infty} R^{4} d R F_{k_{I} 2}^{t r}(R) \sum_{\mu_{0} q_{I}}\left(1 \mu_{0} k_{I} q_{I} \mid 2-q\right) \tau_{1 \mu_{0}}\left(\boldsymbol{\sigma}_{0}\right)\left\langle I M_{f}\left|\tau_{k_{I} q_{I}}(\boldsymbol{I})\right| I M_{i}\right\rangle(-)^{q} \\
=2 \sqrt{\frac{8 \pi}{5}} \int_{0}^{\infty} x^{4} d x v_{p p}^{t r}(x) \iint d \boldsymbol{r}_{1} d \boldsymbol{r}_{2} \Psi_{I, M_{f}}^{*}\left(\boldsymbol{r}_{1}, \boldsymbol{r}_{2}\right)(-)^{\mu} \tau_{2-\mu}(\boldsymbol{S}) \Psi_{I, M_{i}}\left(\boldsymbol{r}_{1}, \boldsymbol{r}_{2}\right) \\
=2 \sqrt{\frac{8 \pi}{5}} \int_{0}^{\infty} x^{4} d x v_{p p}^{t r}(x) \times B_{k_{I}}^{t r} \sum_{\mu_{0} q_{I}}\left(1 \mu_{0} k_{I} q_{I} \mid 2-q\right) \tau_{1 \mu_{0}}\left(\boldsymbol{\sigma}_{0}\right)\left\langle I M_{f}\left|\tau_{k_{I} q_{I}}(\boldsymbol{I})\right| I M_{i}\right\rangle(-)^{q} \\
\Rightarrow \int_{0}^{\infty} R^{4} d R F_{k_{I} 2}^{t r}(R)=2 \sqrt{\frac{8 \pi}{5}} \int_{0}^{\infty} x^{4} d x v_{p p}^{t r}(x) \times B_{k_{I}}^{t r}=2 \sqrt{\frac{8 \pi}{5}} 120 \sum_{j=1}^{N} V_{j}^{T R}\left(R_{j}^{T R}\right)^{7} \times B_{k_{I}}^{t r} .
\end{gathered}
$$

The coefficient $B_{k_{I}}^{t r}$, calculated using the methods described in Appendix $\mathrm{C}$ and the wave- 
functions detailed in Section 3.3.1, is given by

$$
\begin{aligned}
& B_{1}^{t r}=\sqrt{\frac{3}{2}} \hat{j}_{1}^{\prime} \hat{j}_{1} \hat{I} W\left(j_{1} \frac{1}{2} j_{1}^{\prime} \frac{1}{2} ; \ell_{1} I\right) W\left(1 j_{1} I j_{2} ; j_{1}^{\prime} I\right), \\
& B_{3}^{t r}=0 .
\end{aligned}
$$

Therefore,

$$
\int R^{4} d R F_{32}^{t r}(R)=0
$$

For $k_{I}=3$ or 5 and $k=4$, to obtain a non zero result, the folded potential is multiplied by $R^{4} Y_{4 q^{\prime}}^{*}(\hat{\boldsymbol{R}})$ then integrated over all space:

$$
\begin{aligned}
\int d \boldsymbol{R} R^{4} Y_{4 q^{\prime}}^{*}(\hat{\boldsymbol{R}}) F_{k_{I}}^{t r}(R)\left\langle I M_{f}\left|\boldsymbol{S}_{k_{I}}\right| I M_{i}\right\rangle=2 \sqrt{\frac{8 \pi}{5}} \int d \boldsymbol{x} v_{p p}^{t r}(x) \sum_{\mu} Y_{2 \mu}(\hat{\boldsymbol{x}}) \\
\quad \times \iint d \boldsymbol{r}_{1} d \boldsymbol{r}_{2} \Psi_{I, M_{f}}^{*}\left(\boldsymbol{r}_{1}, \boldsymbol{r}_{2}\right)\left(\left|\boldsymbol{x}+\boldsymbol{r}_{1}\right|\right)^{4} Y_{4 q^{\prime}}^{*}\left(\boldsymbol{x} \hat{+} \boldsymbol{r}_{1}\right)(-)^{\mu} \tau_{2-\mu}(\boldsymbol{S}) \Psi_{I, M_{i}}\left(\boldsymbol{r}_{1}, \boldsymbol{r}_{2}\right) .
\end{aligned}
$$

Just as $R^{2} Y_{2 q^{\prime}}^{*}(\hat{\boldsymbol{R}})$ can be expanded using Eq. $3.37, R^{4} Y_{4 q^{\prime}}^{*}(\hat{\boldsymbol{R}})$ is given by

$$
\begin{aligned}
\left(\left|\boldsymbol{x}+\boldsymbol{r}_{1}\right|\right)^{4} Y_{4 q^{\prime}}^{*}\left(\boldsymbol{x} \hat{+} \boldsymbol{r}_{1}\right) & =x^{4} Y_{4 q^{\prime}}^{*}(\hat{\boldsymbol{x}})+r_{1}^{4} Y_{4 q^{\prime}}^{*}\left(\hat{\boldsymbol{r}}_{1}\right) \\
& +\sqrt{4 \pi} 2 \sqrt{3}\left\{\sum_{\alpha \beta}\left(3 \alpha 1 \beta \mid 2 q^{\prime}\right) x^{3} Y_{3 \alpha}^{*}(\hat{\boldsymbol{x}}) r_{1} Y_{1 \beta}^{*}\left(\hat{\boldsymbol{r}}_{1}\right)\right. \\
& \left.+\sum_{\alpha \beta}\left(1 \alpha 3 \beta \mid 2 q^{\prime}\right) x Y_{1 \alpha}^{*}(\hat{\boldsymbol{x}}) r_{1}^{3} Y_{3 \beta}^{*}\left(\hat{\boldsymbol{r}}_{1}\right)\right\} \\
& +\sqrt{4 \pi} \sqrt{\frac{126}{5}} \sum_{\alpha \beta}\left(2 \alpha 2 \beta \mid 2 q^{\prime}\right) x^{2} Y_{2 \alpha}^{*}(\hat{\boldsymbol{x}}) r_{1}^{2} Y_{2 \beta}^{*}\left(\hat{\boldsymbol{r}}_{1}\right)
\end{aligned}
$$

This expansion is taken from Appendix VI of [42]. Of these five terms, only the last one leads to a non zero result when substituted into the right hand side of Eq. 3.76 and integrated over all angles. Therefore, using this term in Eq. 3.76 results in

$$
\begin{aligned}
\int d \boldsymbol{R} & R^{4} Y_{4 q^{\prime}}^{*}(\hat{\boldsymbol{R}}) F_{k_{I} 4}^{t r}(R) \sum_{\mu_{0} q_{I} q}\left(1 \mu_{0} k_{I} q_{I} \mid 4-q\right) \tau_{1 \mu_{0}}\left(\boldsymbol{\sigma}_{0}\right)\left\langle I M_{f}\left|\tau_{k_{I} q_{I}}(\boldsymbol{I})\right| I M_{i}\right\rangle Y_{4 q}(\hat{\boldsymbol{R}})(-)^{q} \\
= & 2 \sqrt{\frac{8 \pi}{5}} \sqrt{4 \pi} \sqrt{\frac{126}{5}} \int d \boldsymbol{x} v_{p p}^{t r}(x) \sum_{\mu \alpha \beta} x^{2} Y_{2 \alpha}^{*}(\hat{\boldsymbol{x}}) Y_{2 \mu}(\hat{\boldsymbol{x}})\left(2 \alpha 2 \beta \mid 2 q^{\prime}\right) \\
& \times \iint d \boldsymbol{r}_{1} d \boldsymbol{r}_{2} \Psi_{I, M_{f}}^{*}\left(\boldsymbol{r}_{1}, \boldsymbol{r}_{2}\right)(-)^{\mu} \tau_{2-\mu}(\boldsymbol{S}) r_{1}^{2} Y_{2 \beta}^{*}\left(\hat{\boldsymbol{r}}_{1}\right) \Psi_{I, M_{i}}\left(\boldsymbol{r}_{1}, \boldsymbol{r}_{2}\right)
\end{aligned}
$$

where the explicit form of $\left\langle I M_{f}\left|S_{k_{I}}\right| I M_{i}\right\rangle$ has been substituted in using Eq. 3.30. Inte- 
grating over all angles $d \Omega_{R}$ and $d \Omega_{x}$ leads to

$$
\begin{aligned}
& \int_{0}^{\infty} R^{6} d R F_{k_{I} 4}^{t r}(R) \sum_{\mu_{0} q_{I} q}\left(1 \mu_{0} k_{I} q_{I} \mid 4-q\right) \tau_{1 \mu_{0}}\left(\boldsymbol{\sigma}_{0}\right)\left\langle I M_{f}\left|\tau_{k_{I} q_{I}}(\boldsymbol{I})\right| I M_{i}\right\rangle(-)^{q} \\
& =2 \sqrt{\frac{8 \pi}{5}} \sqrt{4 \pi} \sqrt{\frac{126}{5}} \int_{0}^{\infty} x^{4} d x v_{p p}^{t r}(x) \sum_{\mu \beta}\left(2 \mu 2 \beta \mid 2 q^{\prime}\right) \\
& \quad \times \iint d \boldsymbol{r}_{1} d \boldsymbol{r}_{2} \Psi_{I, M_{f}}^{*}\left(\boldsymbol{r}_{1}, \boldsymbol{r}_{2}\right)(-)^{\mu} \tau_{2-\mu}(\boldsymbol{S}) r_{1}^{2} Y_{2 \beta}^{*}\left(\hat{\boldsymbol{r}}_{1}\right) \Psi_{I, M_{i}}\left(\boldsymbol{r}_{1}, \boldsymbol{r}_{2}\right) \\
& =2 \sqrt{\frac{8 \pi}{5}} \int_{0}^{\infty} x^{4} d x v_{p p}^{t r}(x) \times C_{k_{I}}^{t r} \sum_{\mu_{0} q_{I} q}\left(1 \mu_{0} k_{I} q_{I} \mid 4-q\right) \tau_{1 \mu_{0}}\left(\boldsymbol{\sigma}_{0}\right)\left\langle I M_{f}\left|\tau_{k_{I} q_{I}}(\boldsymbol{I})\right| I M_{i}\right\rangle(-)^{q} \\
& \Rightarrow \int_{0}^{\infty} R^{6} d R F_{34}^{t r}(R)=2 \sqrt{\frac{8 \pi}{5}} \int_{0}^{\infty} x^{4} d x v_{p p}^{t r}(x) \times C_{k_{I}}^{t r}=2 \sqrt{\frac{8 \pi}{5}} 120 \sum_{j=1}^{N} V_{j}^{T R}\left(R_{j}^{T R}\right)^{7} \times C_{k_{I}}^{t r}
\end{aligned}
$$

As with the other coefficients, $C_{k_{I}}^{t r}$ can be found using the valence nucleon wavefunctions described in Section 3.3.1 and the recoupling techniques detailed in Appendix C:

$$
\begin{aligned}
C_{3}^{t r} & =63 \sqrt{5} \hat{\ell}_{1}^{\prime} \hat{j}_{1}^{\prime} \hat{j}_{1} \hat{I}\left(\ell_{1}^{\prime} 020 \mid \ell_{1} 0\right) W(1142 ; 23) W\left(3 j_{1}^{\prime} I j_{2} ; j_{1} I\right) \\
& \times\left\{\begin{array}{ccc}
2 & 1 & 3 \\
\ell_{1}^{\prime} & \frac{1}{2} & j_{1}^{\prime} \\
\ell_{1} & \frac{1}{2} & j_{1}
\end{array}\right\} \int_{0}^{\infty} r_{1}^{4} d r_{1} u_{\ell_{1}^{\prime} j_{1}^{\prime}}\left(r_{1}\right) u_{\ell_{1} j_{1}}\left(r_{1}\right) \\
C_{5}^{t r} & =0
\end{aligned}
$$




\section{Chapter 4}

\section{Nucleon-Nucleon Interaction}

The bare nucleon-nucleon (NN) interaction is too strong to be used directly in scattering calculations. Realistic forms of bare nuclear forces show strong short range repulsion (hard core) and are too strong to be treated by perturbation theory or other many-body techniques [65]. Also, a nucleon in a nucleus feels the effect of all the other nucleons, i.e. the nuclear medium causes an average potential which all the nucleons are influenced by and contribute to. These complications have led to the use of effective NN interactions that replaces the many-body problem by a simpler two body problem: two nucleons, interacting through a potential, within a many body system.

\subsection{Effective Nucleon-Nucleon Interaction}

Two examples of phenomenological NN interactions are the Hamada Johnston (HJ) potential [66], and the Reid soft core (Reid68) potential [67]. The HJ interaction is an energy independent (but non-relativistic) NN potential that uses a hard core model (i.e. $V(r)=\infty$ for $r<r_{c}=0.343 \mathrm{fm}$ ), with a superposition of Yukawa forms fitted to twonucleon data below $315 \mathrm{MeV}$. The Reid68 interaction uses a soft core (i.e. $V(r)=\infty$ only for $r \rightarrow 0$ ) Yukawa potential fitted to phase parameters and low energy data (below $\sim 350 \mathrm{MeV}$ ).

A method of deriving an effective interaction from the bare NN interaction is known as the Brueckner G-matrix approach, (see [68-70] and references in [71]). The Schrödinger equation for two nucleons/fermions in a Fermi gas is called the Bethe-Goldstone equation [72]. This equation is solved, yielding a G-matrix that is weaker and more smoothly behaved than the bare interaction [65]. This G-matrix can then be used as an effective interaction for two nucleons in a nuclear medium. The G-matrix also takes into account Pauli blocking and nuclear binding and can therefore be used to reflect many-body effects [71]. 
One effective NN interaction which is derived using this method is the density independent M3Y interaction [73]. As described previously, the Bethe-Goldstone equation is solved, in this case using the phenomenological NN potentials of HJ and Reid68, to yield their Brueckner G-matrix elements. A sum of Yukawa terms is then fitted so that the matrix elements reproduce the G-matrix elements. The central parts of the interaction were fitted using three Yukawa terms (hence the name M3Y - with the M standing for Michigan). Further details are given in [73,74]. Density-dependent corrections for the central parts of this interaction have also been made [21,74].

This work was later extended and an effective NN M3Y interaction was derived from the Paris [75] NN potential [76]. The Paris potential was taken to be more fundamental that the HJ and Reid68 interactions as only the short-range part was fitted phenomenologically (to newer data); the medium- and long-range parts were based upon meson theory of nuclear forces [76]. However, there are still an estimated 60 free parameters that are fitted to the data [77], most of which determine the short range part of the potential. While this has the advantage of providing good fits to the data, it obscures the theory underlying the potential. The matrix elements obtained from the Paris potential were not significantly different to those derived from Reid68 [76].

At sufficiently high nucleon bombarding energies (above $\sim 100 \mathrm{MeV}$ ), the G-matrix approaches the t-matrix for free NN scattering and the impulse approximation (where each scattering event is taken to be between free nucleons) is applicable. An effective interaction based on the free NN t-matrix would therefore consider a projectile nucleon scattering from one target nucleon that is under the influence of the surrounding nuclear medium. Love and Franey [78] use this method to derive an energy-dependent free NN interaction fitted to NN amplitudes for energies between 100 and $800 \mathrm{MeV}$. They also used a sum of Yukawa forms to represent the radial parts of their interactions.

Effective interactions of this type, that are based on the realistic bare NN interaction, but use some kind of G- or t-matrix, are known as 'realistic'. To ensure that these effective forces reproduce experimental data, they depend on parameters that are adjusted to fit NN data. Therefore, most microscopic descriptions of the nucleus that use effective interactions are based on the known NN force, but are still in some sense phenomenological.

Microscopic calculations of spin-spin interactions have used several different types of NN interactions. Satchler [9] used the HJ potential [79]. Dabrowski and Haensel [11] used the Reid68 [67], while both Petrovich et al. [17] and McAbee [20] use the effective M3Y [73] interactions. An effective interaction derived from the Paris NN potential [75] was used in the work of von Przewoski et al. [15] for the microscopic parts of their calculation.

The NN interaction used in this work is based on a realistic free-space NN interaction that is an improved version of the Bonn-B potential (see references in [71]). The Bonn 
potential goes further into meson theory than the Paris potential, which makes the shortrange phenomenology (a weakness of the Paris potential) unnecessary. The Bonn model has 12 parameters for which physically reasonable choices are made and result in a potential that gives a satisfactory description of NN data up to $300 \mathrm{MeV}$ [77]. The effective NN interaction is obtained from the updated Bonn-B potential using the Brueckner G-matrix approach as described in [71]. The Yukawa parameters used here are calculated from the effective NN interaction as described in [71] and were provided by [80]. The parameters are fitted for a free complex t-matrix and are calculated for an incident proton laboratory energy of $200 \mathrm{MeV}$, with the target at rest. The Yukawa parameters are listed in Tables E.1 and E.2 in Appendix E.

\subsection{Spin- and Isospin- Structure of the Effective NN Interaction}

The effective NN interaction between nucleon $a$, and nucleon $b$, can be written in isospin formalism as the sum of central (CE), spin-orbit (LS) and tensor (TR) terms [44]

$$
\begin{aligned}
V_{N N}(a, b) & =v_{00}^{C E}(r)+v_{10}^{C E}(r) \boldsymbol{\sigma}_{a} \cdot \boldsymbol{\sigma}_{b}+v_{01}^{C E}(r) \boldsymbol{\tau}_{a} \cdot \boldsymbol{\tau}_{b}+v_{11}^{C E}(r) \boldsymbol{\sigma}_{a} \cdot \boldsymbol{\sigma}_{b} \boldsymbol{\tau}_{a} \cdot \boldsymbol{\tau}_{b} \\
& +\left[v_{10}^{L S}(r)+v_{11}^{L S}(r) \boldsymbol{\tau}_{a} \cdot \boldsymbol{\tau}_{b}\right] \ell_{a b} \cdot\left(\boldsymbol{\sigma}_{a}+\boldsymbol{\sigma}_{b}\right) \\
& +\left[v_{10}^{T R}(r)+v_{11}^{T R}(r) \boldsymbol{\tau}_{a} \cdot \boldsymbol{\tau}_{b}\right] \boldsymbol{S}_{a b}
\end{aligned}
$$

where $r=\left|\boldsymbol{r}_{a}-\boldsymbol{r}_{b}\right|$, is the separation between the two nucleons, and the relative angular momentum is

$$
\ell_{a b}=(2 \hbar)^{-1} \boldsymbol{r} \times\left(\boldsymbol{p}_{a}-\boldsymbol{p}_{b}\right),
$$

where $\boldsymbol{p}$ is the nucleon momentum. The NN tensor operator is

$$
\boldsymbol{S}_{a b}=3\left(\boldsymbol{\sigma}_{a} \cdot \hat{\boldsymbol{r}}\right)\left(\boldsymbol{\sigma}_{b} \cdot \hat{\boldsymbol{r}}\right)-\boldsymbol{\sigma}_{a} \cdot \boldsymbol{\sigma}_{b}
$$

The isospin operators $\boldsymbol{\tau}$ are 2x2 matrices analogous to the Pauli spin operator, $\boldsymbol{\sigma}$ [81]. They add to give a total isospin, $\boldsymbol{T}=\frac{1}{2}\left(\boldsymbol{\tau}_{a}+\boldsymbol{\tau}_{b}\right)$, just as the total spin, $\boldsymbol{S}$, is given by $\boldsymbol{S}=\frac{1}{2}\left(\sigma_{a}+\sigma_{b}\right)^{1}$. The terms in Eq. 4.1 have the spin-isospin structure $v_{s t}^{C E}$ and result in spin transfer, $s$ and isospin transfer, $t$. The spin/isospin-independent term is therefore $v_{00}^{C E}(r)$ and the spin independent isovector term is $v_{01}^{C E}(r) \boldsymbol{\tau}_{a} \cdot \boldsymbol{\tau}_{b}$ etc. The spin-orbit, $v_{s t}^{L S}(r)$ and tensor, $v_{s t}^{T R}(r)$ terms all have spin transfer $s=1$ and only act on triplet (total

\footnotetext{
${ }^{1}$ The components of the isospin operators also share the property $\tau_{1}^{2}=\tau_{2}^{2}=\tau_{3}^{2}=1$ with the Pauli spin operators. For $T=0, \tau_{a} \cdot \tau_{b}=-3$ and for $T=1, \tau_{a} \cdot \tau_{b}=1$, just as for total spin, $S=0$, (singlet state), $\sigma_{a} \cdot \sigma_{b}=-3$ and for, $S=1$, (triplet state), $\sigma_{a} \cdot \sigma_{b}=1$.
} 
spin $S=1$ ) states.

The two NN interactions used in this work are $V_{p p}$ and $V_{p n}$ corresponding to a proton (a) - proton (b) and proton (a) - neutron (b) interaction, respectively. This results in two different versions of Eq. 4.1:

$$
\begin{aligned}
V_{p p}(a, b) & =V_{p p}^{T=1}(a, b) \\
& =\left[v_{00}^{C E}(r)+v_{01}^{C E}(r)\right]+\left[v_{10}^{C E}(r)+v_{11}^{C E}(r)\right] \boldsymbol{\sigma}_{a} \cdot \boldsymbol{\sigma}_{b} \\
& +\left[v_{10}^{L S}(r)+v_{11}^{L S}(r)\right] \ell_{a b} \cdot\left(\boldsymbol{\sigma}_{a}+\boldsymbol{\sigma}_{b}\right)+\left[v_{10}^{T R}(r)+v_{11}^{T R}(r)\right] \boldsymbol{S}_{a b}, \\
V_{p n}(a, b) & =\frac{1}{2}\left[V_{p n}^{T=0}(a, b)+V_{p n}^{T=1}(a, b)\right] \\
& =\left[v_{00}^{C E}(r)-v_{01}^{C E}(r)\right]+\left[v_{10}^{C E}(r)-v_{11}^{C E}(r)\right] \boldsymbol{\sigma}_{a} \cdot \boldsymbol{\sigma}_{b} \\
& +\left[v_{10}^{L S}(r)-v_{11}^{L S}(r)\right] \ell_{a b} \cdot\left(\boldsymbol{\sigma}_{a}+\boldsymbol{\sigma}_{b}\right)+\left[v_{10}^{T R}(r)-v_{11}^{T R}(r)\right] \boldsymbol{S}_{a b},
\end{aligned}
$$

which can be simplified to

$$
V_{N N}(a, b)=v_{N N}^{c e}(r)+v_{N N}^{\sigma \sigma}(r) \sigma_{a} \cdot \sigma_{b}+v_{N N}^{l s}(r) \ell_{a b} \cdot\left(\sigma_{a}+\sigma_{b}\right)+v_{N N}^{t r}(r) \boldsymbol{S}_{a b}
$$

where the $v_{N N}(r)$ components are different for like and unlike particles.

The $v_{s t}$ components in Eq. 4.1 can be expressed in terms of total spin, singlet, $S$, and triplet, $T$, of the two-nucleon system and its relative angular momentum, even, $E$, and odd, $O,[44]$

$$
\begin{aligned}
v_{00}^{C E}(r) & =\left[3 v_{T E}^{C E}(r)+3 v_{S E}^{C E}(r)+9 v_{T O}^{C E}(r)+v_{S O}^{C E}(r)\right] / 16 \\
v_{10}^{C E}(r) & =\left[v_{T E}^{C E}(r)-3 v_{S E}^{C E}(r)+3 v_{T O}^{C E}(r)-v_{S O}^{C E}(r)\right] / 16, \\
v_{01}^{C E}(r) & =\left[-3 v_{T E}^{C E}(r)+v_{S E}^{C E}(r)+3 v_{T O}^{C E}(r)-v_{S O}^{C E}(r)\right] / 16, \\
v_{11}^{C E}(r) & =\left[-v_{T E}^{C E}(r)-v_{S E}^{C E}(r)+v_{T O}^{C E}(r)+v_{S O}^{C E}(r)\right] / 16, \\
v_{10}^{L S}(r) & =\left[v_{T E}^{L S}(r)+3 v_{T O}^{L S}(r)\right] / 4 \\
v_{11}^{L S}(r) & =\left[-v_{T E}^{L S}(r)+v_{T O}^{L S}(r)\right] / 4 \\
v_{10}^{T R}(r) & =\left[v_{T E}^{T R}(r)+3 v_{T O}^{T R}(r)\right] / 4 \\
v_{11}^{T R}(r) & =\left[-v_{T E}^{T R}(r)+v_{T O}^{T R}(r)\right] / 4 .
\end{aligned}
$$

The $v_{N N}$ components from Eq. 4.6 can also be expressed in this way, for like particles

$$
\begin{aligned}
v_{p p}^{c e}(r) & =\left[v_{S E}^{C E}(r)+3 v_{T O}^{C E}(r)\right] / 4 \\
v_{p p}^{\sigma \sigma}(r) & =\left[-v_{S E}^{C E}(r)+v_{T O}^{C E}(r)\right] / 4 \\
v_{p p}^{l s}(r) & =v_{T O}^{L S}(r) \\
v_{p p}^{t r}(r) & =v_{T O}^{T R}(r)
\end{aligned}
$$


and unlike particles

$$
\begin{aligned}
v_{p n}^{c e}(r) & =\left[3 v_{T E}^{C E}(r)+v_{S E}^{C E}(r)+3 v_{T O}^{C E}(r)+v_{S O}^{C E}(r)\right] / 8 \\
v_{p n}^{\sigma \sigma}(r) & =\left[v_{T E}^{C E}(r)-v_{S E}^{C E}(r)+v_{T O}^{C E}(r)-v_{S O}^{C E}(r)\right] / 8 \\
v_{p n}^{l s}(r) & =\left[v_{T E}^{L S}(r)+v_{T O}^{L S}(r)\right] / 2 \\
v_{p n}^{t r}(r) & =\left[v_{T E}^{T r}(r)+v_{T O}^{T R}(r)\right] / 2 .
\end{aligned}
$$

\subsection{Parametrisation}

The parametrisation used in this work is the same as that detailed in Love and Franey [78]. For computational simplicity the radial parts of the singlet/triplet, odd/even $v$ components are taken to be a sum of Yukawa forms

$$
\begin{aligned}
v_{S / T, O / E}^{C E}(r) & =\sum_{j=1}^{N_{C E}} V_{j}^{C E} Y\left(r / R_{j}^{C E}\right) ; \quad Y(x)=e^{-x} / x \\
v_{O / E}^{L S}(r) & =\sum_{j=1}^{N_{L S}} V_{j}^{L S} Y\left(r / R_{j}^{L S}\right) \\
v_{O / E}^{T R}(r) & =\sum_{j=1}^{N_{T R}} V_{j}^{T R} r^{2} Y\left(r / R_{j}^{T R}\right),
\end{aligned}
$$

where the $V_{j}$ 's are complex strengths in $\mathrm{MeV}\left(\mathrm{MeV} \cdot \mathrm{fm}^{-2}\right.$ for the tensor terms) and the $R_{j}$ 's are the range parameters. The parameters are listed in Tables E.1 and E.2 in Appendix E. These equations yield the direct part of the effective interaction.

\subsection{Exchange Terms}

For the case of nucleon-nucleus scattering, the Pauli principle requires the total nuclear wavefunction to be antisymmetric under the interchange of nucleons between the projectile and target. The consequences of this requirement have been studied within the framework of the resonating-group method (RGM) within an elastic channel. RGM is a microscopic method used to solve nuclear many body problems based on the idea that nucleons in nuclei spend a fraction of their time in clusters. For a good description of RGM see [82]. The RGM uses totally antisymmetrised wavefunctions and can therefore be used to investigate the effects of the Pauli principle.

For nucleus-nucleus elastic scattering, the antisymmetrisation of the wavefunction using RGM yields a local direct term and various nonlocal nucleon-exchange terms, de- 
pending on the number of nucleons interchanged [74]. In all scattering systems the most important exchange contributions come from one-nucleon exchange and core-exchange (the complete exchange of all the nucleons from the smaller system), although the latter is only important when the two nuclei have a similar number of nucleons [83]. So for nucleon-nucleus scattering it is reasonable when considering exchange corrections arising from antisymmetrisation to use single-nucleon exchange (SNE) or, specifically, singlenucleon knock-on exchange (SNKE), where a target nucleon is ejected and replaced by the projectile nucleon after their interaction [44]. To formally include knock-on exchange in a folding model the effective NN interaction potential term, $v_{p t}(r)$, between projectile nucleon $p$ and target nucleon $t$ can be replaced by $[57,74]$

$$
v_{p t}(r) \rightarrow\left(1-P_{p t}\right) v_{p t}(r)
$$

where $P_{p t}$ is the operator that exchanges all the coordinates of the two nucleons. The exchange operator can be written in terms of the spin and isospin operators $P^{\sigma}$ and $P^{\tau}$ which exchange the spin and isospin coordinates, respectively,

$$
P_{p t}=P_{p t}^{\sigma} P_{p t}^{\tau} P_{p t}^{r}
$$

where $P_{p t}^{r}$ exchanges the spatial coordinates of the two nucleons. Eq 4.13 can now be written as

$$
\begin{aligned}
v_{p t}(r) & \rightarrow\left(1-P_{p t}\right) v_{p t}(r), \\
& \rightarrow v_{p t}(r)+\hat{v}_{p t}(r) P_{p t}^{r},
\end{aligned}
$$

where $\hat{v}_{p t}(r)=-P_{p t}^{\sigma} P_{p t}^{\tau} v_{p t}(r)$ and has a spin-isospin decomposition that is the same as $v_{p t}(r)$ given in Eq. 4.7, except that the sign for the odd-state terms is changed.

The effect of exchange terms on spin-spin interactions derived from folding models has not been fully investigated. Early folding models for spin-spin interactions [7-11] used effective interactions which were mainly phenomenological so exchange was crudely included. However explicit exchange effects were not incorporated, although they were discussed [9]. Petrovich [17] and McAbee [20] used SNKE to approximate exchange for the $v_{N N}^{\sigma \sigma}(r) \sigma_{a} \cdot \sigma_{b}$ term in their effective NN interaction. Petrovich also approximates the spin-orbit exchange terms using the odd state spin-orbit components alone, but both works neglected exchange effects for the tensor terms in their final calculations. It is one of the goals of this work to consistently include, for the first time, SNKE terms for both the central $v_{N N}^{\sigma \sigma}(r) \sigma_{a} \cdot \sigma_{b}$ and tensor $v_{N N}^{t r}(r) S_{a b}$ terms in the effective NN interaction and investigate their contributions to the spin-spin interactions. 


\subsection{Zero-Range Pseudo-Potentials}

In order to avoid the complicated evaluation of exact exchange amplitudes, the use of zero-range pseudo-potentials has been adopted for treating exchange terms in folding models [84-86]. In the simplest of these approximate treatments the exchange pseudopotentials are derived to yield the same Born amplitudes as their equivalent exchange terms $[44,83]$. The resulting zero-range effective exchange interaction can then be used to evaluate the scattering amplitude more readily than for the full exchange terms.

\subsubsection{Central Exchange}

In the NN interactions given in Eq. 4.6 between projectile nucleon $a$ and target nucleon $b$, the central term $v_{N N}^{\sigma \sigma}(r) \sigma_{a} \cdot \sigma_{b}$ is now given by a direct and SNKE term (from Eq. 4.16) of the form

$$
v_{N N}^{\sigma \sigma}(r) \sigma_{a} \cdot \sigma_{b} \rightarrow v_{N N}^{\sigma \sigma}(r) \sigma_{a} \cdot \sigma_{b}+\hat{v}_{N N}^{\sigma \sigma}(r) \sigma_{a} \cdot \sigma_{b} P_{a b}^{r}
$$

The Born amplitude (taking the incident and scattered wavefunction as plane waves) for this exchange term is given by

$$
\left\langle\boldsymbol{k}^{\prime}\left|\hat{v}_{N N}^{\sigma \sigma}(r) \boldsymbol{\sigma}_{a} \cdot \boldsymbol{\sigma}_{b} P_{a b}^{r}\right| \boldsymbol{k}\right\rangle=4 \pi \int_{0}^{\infty} j_{0}(Q r) \hat{v}_{N N}^{\sigma \sigma}(r) r^{2} d r \boldsymbol{\sigma}_{a} \cdot \boldsymbol{\sigma}_{b}
$$

in terms of the spherical Bessel function of the first kind, $j_{0}(Q r)$, where $Q$ is the sum of the initial and final wave number of either particle in the centre of mass system,

$$
Q=k^{\prime}+k
$$

The value taken for $Q$ is discussed in Section 4.5.3. The zero-range pseudo-potential used to approximate this exchange term is given by

$$
\hat{v}_{N N}^{\sigma \sigma}(r) \boldsymbol{\sigma}_{a} \cdot \boldsymbol{\sigma}_{b} P_{a b}^{r} \rightarrow \hat{J}_{\sigma \sigma}(Q) \delta(\boldsymbol{r}) \boldsymbol{\sigma}_{a} \cdot \boldsymbol{\sigma}_{b} P_{a b}^{r}
$$

where the strength $\hat{J}_{\sigma \sigma}(Q)$ is the Fourier transform of $\hat{v}_{N N}^{\sigma \sigma}(r) P_{a b}^{r}$

$$
\hat{J}_{\sigma \sigma}(Q)=4 \pi \int_{0}^{\infty} j_{0}(Q r) \hat{v}_{N N}^{\sigma \sigma}(r) r^{2} d r
$$

as in Eq. 4.18. This pseudo-potential for the central exchange contribution to the NN interaction has been used by several authors and has been shown to be quite accurate for nucleon scattering at energies above $100 \mathrm{MeV}$ (see references in $[44,57,74]$ ). 


\subsubsection{Tensor Exchange}

In previous works on spin-spin interactions, the contribution of the tensor exchange term in the NN interaction has been neglected due to the difficulty in taking the pseudopotential approach for the noncentral tensor potential [21]. In this work the tensor exchange will be included using the pseudo-potential approximation in a process analogous to that described in the previous section for the central exchange. This will complicate the final calculation of the scattering amplitude but will enable the first estimate of the effect on spin-spin interactions of the tensor exchange term in the NN interaction.

The SNKE approximation (Eq. 4.16) for the direct tensor term $v_{N N}^{t r}(r) \boldsymbol{S}_{a b}$ in the NN interaction is given by

$$
v_{N N}^{t r}(r) \boldsymbol{S}_{a b} \rightarrow v_{N N}^{t r}(r) \boldsymbol{S}_{a b}+\hat{v}_{N N}^{t r}(r) \boldsymbol{S}_{a b} P_{a b}^{r}
$$

The Born amplitude for the tensor exchange term is

$$
\left\langle\boldsymbol{k}^{\prime}\left|\hat{v}_{N N}^{t r}(r) \boldsymbol{S}_{a b}\left(\boldsymbol{\sigma}_{a}, \boldsymbol{\sigma}_{b}, \hat{\boldsymbol{r}}\right) P_{a b}^{r}\right| \boldsymbol{k}\right\rangle=-4 \pi \int_{0}^{\infty} j_{2}(Q r) \hat{v}_{N N}^{t r}(r) r^{2} d r \boldsymbol{S}_{a b}\left(\boldsymbol{\sigma}_{a}, \boldsymbol{\sigma}_{b}, \hat{\boldsymbol{Q}}\right),
$$

where the tensor operator, $\boldsymbol{S}_{a b}\left(\boldsymbol{\sigma}_{a}, \boldsymbol{\sigma}_{b}, \hat{\boldsymbol{Q}}\right)$, is now given by

$$
\boldsymbol{S}_{a b}\left(\boldsymbol{\sigma}_{a}, \boldsymbol{\sigma}_{b}, \hat{\mathbf{Q}}\right)=3\left(\boldsymbol{\sigma}_{a} \cdot \hat{\mathbf{Q}}\right)\left(\boldsymbol{\sigma}_{b} \cdot \hat{\mathbf{Q}}\right)-\boldsymbol{\sigma}_{a} \cdot \boldsymbol{\sigma}_{b}
$$

Defining the operator $\hat{\boldsymbol{S}}_{a b}\left(\boldsymbol{\sigma}_{a}, \boldsymbol{\sigma}_{b}, \boldsymbol{Q}\right)=Q^{2} \boldsymbol{S}_{a b}\left(\boldsymbol{\sigma}_{a}, \boldsymbol{\sigma}_{b}, \hat{\boldsymbol{Q}}\right)$ and using

$$
\left\langle\boldsymbol{k}^{\prime}\left|\hat{\boldsymbol{S}}_{a b}\left(\boldsymbol{\sigma}_{a}, \boldsymbol{\sigma}_{b}, \boldsymbol{Q}\right)\right| \boldsymbol{k}\right\rangle=\left\langle\boldsymbol{k}^{\prime}\left|\hat{\boldsymbol{S}}_{a b}\left(\boldsymbol{\sigma}_{a}, \boldsymbol{\sigma}_{b},-\imath \nabla_{\boldsymbol{r}}\right)\right| \boldsymbol{k}\right\rangle,
$$

Eq 4.23 can be modified to a form more suitable for manipulation in the folding model

$$
\left\langle\boldsymbol{k}^{\prime}\left|\hat{v}_{N N}^{t r}(r) \boldsymbol{S}_{a b}\left(\boldsymbol{\sigma}_{a}, \boldsymbol{\sigma}_{b}, \hat{\boldsymbol{r}}\right) P_{a b}^{r}\right| \boldsymbol{k}\right\rangle=-\frac{4 \pi}{Q^{2}} \int_{0}^{\infty} j_{2}(Q r) \hat{v}_{N N}^{t r}(r) r^{2} d r \hat{\boldsymbol{S}}_{a b}\left(\boldsymbol{\sigma}_{a}, \boldsymbol{\sigma}_{b},-\imath \nabla_{\boldsymbol{r}}\right) .
$$

The zero-range pseudo-potential used to approximate this term is

$$
\hat{v}_{N N}^{t r}(r) \boldsymbol{S}_{a b} P_{a b}^{r} \rightarrow \hat{J}_{t r}(Q)\left[\hat{\boldsymbol{S}}_{a b}\left(\boldsymbol{\sigma}_{a}, \boldsymbol{\sigma}_{b},-\imath \nabla_{\boldsymbol{r}}\right) \delta(\boldsymbol{r})\right] P_{a b}^{r},
$$

where the strength $\hat{J}_{t r}(Q)$ is calculated using the Fourier transform

$$
\hat{J}_{t r}(Q)=-\frac{4 \pi}{Q^{2}} \int_{0}^{\infty} j_{2}(Q r) \hat{v}_{N N}^{t r}(r) r^{2} d r .
$$

The form of the tensor exchange term given in 4.27 is non-local, which complicates the 
calculation of the folded potential and observables. The details of how the non-locality of this exchange interaction affects the folding model and the calculation of the scattering amplitude will be given in the next chapter.

\subsubsection{Approximation for $\mathrm{Q}$}

The exchange terms change the dependence of the Born amplitudes from the momentum transfer $\boldsymbol{q}=\boldsymbol{k}^{\prime}-\boldsymbol{k}$ to $\boldsymbol{Q}=\boldsymbol{k}^{\prime}+\boldsymbol{k}$, through the action of the $P_{a b}^{r}$ exchange operator which interchanges the spatial coordinates of the two nucleons

$$
\begin{aligned}
\left\langle\boldsymbol{k}^{\prime}\left|v_{N N}\right| \boldsymbol{k}\right\rangle & =\int d \boldsymbol{r} e^{-\imath \boldsymbol{k}^{\prime} \cdot \boldsymbol{r}} v_{N N}(r) e^{\imath \boldsymbol{k} \cdot \boldsymbol{r}}=\int d \boldsymbol{r} v_{N N}(r) e^{-\imath \boldsymbol{q} \cdot \boldsymbol{r}} \\
\left\langle\boldsymbol{k}^{\prime}\left|\hat{v}_{N N} P_{a b}^{r}\right| \boldsymbol{k}\right\rangle & =\int d \boldsymbol{r} e^{-\imath \boldsymbol{k}^{\prime} \cdot \boldsymbol{r}} \hat{v}_{N N}(r) e^{-\imath \boldsymbol{k} \cdot \boldsymbol{r}}=\int d \boldsymbol{r} \hat{v}_{N N}(r) e^{-\imath \boldsymbol{Q} \cdot \boldsymbol{r}}
\end{aligned}
$$

In order to evaluate the Born amplitudes for the exchange terms, a value must be adopted for the magnitude of $\boldsymbol{Q}$. The simplest choice is to take this to be the centre-of-mass wave number for the incident particle: $Q=k_{c m}[44,71]$. This choice is reasonable for scattering in the forward direction. However, the measurement of $D_{N N}$ being analysed in this work indicates that the variation of $D_{N N}$ from unity is most significant at large scattering angles. Taking the value of $Q$ associated with a scattering angle of $\theta=0^{\circ}$ would therefore have limited meaning. In order to perform an improved calculation of these exchange terms at large scattering angles a more physical approximations for the magnitude of $\boldsymbol{Q}$, which changes with scattering angle, is required.

\section{Nucleon-Nucleon Scattering}

In the following discussion we will consider the scattering of the incident nucleon with wave number $\boldsymbol{k}_{a}$ by a single valence nucleon with initial wave number $\boldsymbol{k}_{b}$, which for simplicity is bound to a spinless core of infinite mass. The Born amplitude of the local NN interaction term $v_{N N}(r)$, (where $\boldsymbol{r}$ is the relative separation between the two nucleons $\boldsymbol{r}=\boldsymbol{r}_{a}-\boldsymbol{r}_{b}$ ), is given by

$$
\left\langle\boldsymbol{k}_{a}^{\prime}, \boldsymbol{k}_{b}^{\prime}\left|v_{N N}\right| \boldsymbol{k}_{a}, \boldsymbol{k}_{b}\right\rangle=\iint d \boldsymbol{r}_{a} d \boldsymbol{r}_{b} e^{-\imath \boldsymbol{k}_{a}^{\prime} \cdot \boldsymbol{r}_{a}} e^{-\imath \boldsymbol{k}_{b}^{\prime} \cdot \boldsymbol{r}_{b}} v_{N N}(r) e^{\imath \boldsymbol{k}_{a} \cdot \boldsymbol{r}_{a}} e^{\imath \boldsymbol{k}_{b} \cdot \boldsymbol{r}_{b}}
$$

where $\boldsymbol{k}_{a}^{\prime}$ and $\boldsymbol{k}_{b}^{\prime}$ are the final wave numbers of the two nucleons. Using

$$
\boldsymbol{k}_{a} \cdot \boldsymbol{r}_{a}+\boldsymbol{k}_{b} \cdot \boldsymbol{r}_{b}=\frac{\left(\boldsymbol{k}_{a}-\boldsymbol{k}_{b}\right) \cdot\left(\boldsymbol{r}_{a}-\boldsymbol{r}_{b}\right)}{2}+\frac{\left(\boldsymbol{k}_{a}+\boldsymbol{k}_{b}\right) \cdot\left(\boldsymbol{r}_{a}+\boldsymbol{r}_{b}\right)}{2}
$$


and assuming the potential conserves the total momentum of the system, which is assured by using $\delta\left(\boldsymbol{k}_{a}^{\prime}+\boldsymbol{k}_{b}^{\prime}-\boldsymbol{k}_{a}-\boldsymbol{k}_{b}\right)$, leads to

$$
\begin{aligned}
\left\langle\boldsymbol{k}_{a}^{\prime}, \boldsymbol{k}_{b}^{\prime}\left|v_{N N}\right| \boldsymbol{k}_{a}, \boldsymbol{k}_{b}\right\rangle & =\int d \boldsymbol{r} e^{-\imath\left(\boldsymbol{k}_{a}^{\prime}-\boldsymbol{k}_{b}^{\prime}\right) \cdot \boldsymbol{r} / 2} v_{N N}(r) e^{\imath\left(\boldsymbol{k}_{a}-\boldsymbol{k}_{b}\right) \cdot \boldsymbol{r} / 2} \delta\left(\boldsymbol{k}_{a}^{\prime}+\boldsymbol{k}_{b}^{\prime}-\boldsymbol{k}_{a}-\boldsymbol{k}_{b}\right), \\
& =\delta\left(\boldsymbol{k}_{a}^{\prime}+\boldsymbol{k}_{b}^{\prime}-\boldsymbol{k}_{a}-\boldsymbol{k}_{b}\right)\left\langle\frac{\boldsymbol{k}_{a}^{\prime}-\boldsymbol{k}_{b}^{\prime}}{2}\left|v_{N N}\right| \frac{\boldsymbol{k}_{a}-\boldsymbol{k}_{b}}{2}\right\rangle,
\end{aligned}
$$

neglecting factors of $\pi$ for this discussion. The momentum transfer $\boldsymbol{q}$ and vector $\boldsymbol{Q}$, in terms of the individual nucleon momenta can be obtained from this Born amplitude by taking

$$
\boldsymbol{k}^{\prime}=\frac{\boldsymbol{k}_{a}^{\prime}-\boldsymbol{k}_{b}^{\prime}}{2} \quad, \quad \boldsymbol{k}=\frac{\boldsymbol{k}_{a}-\boldsymbol{k}_{b}}{2},
$$

and using the conservation of momentum $\boldsymbol{k}_{a}^{\prime}+\boldsymbol{k}_{b}^{\prime}=\boldsymbol{k}_{a}+\boldsymbol{k}_{b}$ to give

$$
\boldsymbol{q}=\boldsymbol{k}^{\prime}-\boldsymbol{k}=\boldsymbol{k}_{a}^{\prime}-\boldsymbol{k}_{a} \quad, \quad \boldsymbol{Q}=\boldsymbol{k}^{\prime}+\boldsymbol{k}=\boldsymbol{k}_{a}^{\prime}-\boldsymbol{k}_{b}
$$

assuming on-shell scattering, i.e. $k_{a}=k_{a}^{\prime}$. In order to approximate $Q$, an appropriate value of $k_{b}$ must be selected.

\section{Folding Model}

In order to determine which value of $Q$ has the most important contribution to the scattering amplitude, the folded potential will be treated using the Born approximation in momentum space. The folding integral given in Eq. 3.6, which averages the NN interaction $v_{N N}$ over the target nucleon wavefunction $\Psi_{A}\left(\boldsymbol{k}_{b}\right)$, can be written in momentum space as

$$
\left\langle\boldsymbol{k}_{a}^{\prime}, \Psi_{A^{\prime}}\left|v_{N N}\right| \boldsymbol{k}_{a}, \Psi_{A}\right\rangle=\iint d \boldsymbol{k}_{b}^{\prime} d \boldsymbol{k}_{b} \Psi_{A^{\prime}}^{*}\left(\boldsymbol{k}_{b}^{\prime}\right)\left\langle\boldsymbol{k}_{a}^{\prime}, \boldsymbol{k}_{b}^{\prime}\left|v_{N N}\right| \boldsymbol{k}_{a}, \boldsymbol{k}_{b}\right\rangle \Psi_{A}\left(\boldsymbol{k}_{b}\right)
$$

This gives the Born amplitude

$$
\begin{aligned}
& \left\langle\boldsymbol{k}_{a}^{\prime}, \Psi_{A^{\prime}}\left|v_{N N}\right| \boldsymbol{k}_{a}, \Psi_{A}\right\rangle \\
& \quad=\iint d \boldsymbol{k}_{b}^{\prime} d \boldsymbol{k}_{b} \delta\left(\boldsymbol{k}_{a}^{\prime}+\boldsymbol{k}_{b}^{\prime}-\boldsymbol{k}_{a}-\boldsymbol{k}_{b}\right) \Psi_{A^{\prime}}^{*}\left(\boldsymbol{k}_{b}^{\prime}\right)\left\langle\boldsymbol{k}^{\prime}\left|v_{N N}\right| \boldsymbol{k}\right\rangle \Psi_{A}\left(\boldsymbol{k}_{b}\right) \\
& \quad=\int d \boldsymbol{k}_{b} \Psi_{A^{\prime}}^{*}\left(\boldsymbol{k}_{b}-\boldsymbol{q}\right)\left\langle\boldsymbol{k}^{\prime}\left|v_{N N}\right| \boldsymbol{k}\right\rangle \Psi_{A}\left(\boldsymbol{k}_{b}\right)
\end{aligned}
$$

where Eqs. 4.33 and 4.34 have been used. When $v_{N N}$ is local then $\left\langle\boldsymbol{k}^{\prime}\left|v_{N N}\right| \boldsymbol{k}\right\rangle$ is a function of $\boldsymbol{q}$ and can be taken outside of the integrals. For non local potentials, the matrix element of $v_{N N}$ depends on $\boldsymbol{Q}$, which in turn is dependent on $\boldsymbol{k}_{b}$, and cannot be taken out of the $\boldsymbol{k}_{b}$ integral. Therefore to avoid evaluating the $\boldsymbol{k}_{b}$ integral exactly an appropriate value of 
$\boldsymbol{Q}$ will be chosen at which to evaluate $\left\langle\boldsymbol{k}^{\prime}\left|v_{N N}\right| \boldsymbol{k}\right\rangle$.

The most important contribution of $\boldsymbol{k}_{b}$ to this integral is given by the product of the final and initial target wavefunctions $\Psi_{A^{\prime}}^{*}\left(\boldsymbol{k}_{b}-\boldsymbol{q}\right)$ and $\Psi_{A}\left(\boldsymbol{k}_{b}\right)$ respectively. Taking the form of the wavefunctions to be harmonic oscillators (HO) gives, $\Psi_{A}(r)=P(r) \exp \left(-r^{2} / R^{2}\right)$, where $P(r)$ is a polynomial in $r$ and $R$ is the HO parameter. The Fourier transform of this wavefunction is then $\Psi_{A}(K)=P(K) \exp \left(-K^{2} R^{2} / 4\right)$. For large $R$, the polynomials can be neglected and the values of $\boldsymbol{k}_{b}$ that are most important can be found from the maximum of

$$
\exp \left[-\left(\boldsymbol{k}_{b}-\boldsymbol{q}\right)^{2} \frac{R^{2}}{4}\right] \exp \left[-k_{b}^{2} \frac{R^{2}}{4}\right]=\exp \left[-\left(\frac{q^{2}}{2}+2\left[\boldsymbol{k}_{b}-\frac{\boldsymbol{q}}{2}\right]^{2}\right) \frac{R^{2}}{4}\right]
$$

which occurs when $\left(q^{2} / 2+2\left[\boldsymbol{k}_{b}-\boldsymbol{q} / 2\right]^{2}\right) R^{2} / 4$ is at a minimum, i.e. when $\boldsymbol{k}_{b}=\boldsymbol{q} / 2$. For this approximation of $\boldsymbol{Q}$, the value of $\boldsymbol{k}_{b}$ is therefore taken to be

$$
\boldsymbol{Q}=\boldsymbol{k}_{a}^{\prime}-\boldsymbol{k}_{b}=\boldsymbol{k}_{a}^{\prime}-\frac{\boldsymbol{q}}{2}=\frac{\boldsymbol{k}_{a}^{\prime}+\boldsymbol{k}_{a}}{2}
$$

This leads to the approximation for the magnitude of $Q$ used in this work:

$$
Q=k_{a} \cos (\theta / 2)
$$

assuming $k_{a}=k_{a}^{\prime}$ and $\theta$ is the angle between $\boldsymbol{k}_{a}$ and $\boldsymbol{k}_{a}^{\prime}$ [87]. This approximation for $Q$ is the same as that obtained from the prescription described in Appendix B of ref [71], in the limit $A \rightarrow \infty$. 


\section{Chapter 5}

\section{Exchange Folding Model}

In this Chapter details will be given of how the folding model, discussed in Chapter 3, must be modified when using exchange (nonlocal) terms instead of direct (local) terms in the NN interaction. This change requires new formalism to determine the folded spin-spin potentials as well as a modification of the DWBA scattering amplitude derived in Chapter 2. Using the same format as Chapter 3, only the final result of the folding will be given in each section. The full details of the folding formalism for the exchange terms is given in Appendix D.

\subsection{General Folding for Exchange Terms}

The general folding equation derived in Section 3.4 needs to be slightly modified for exchange terms in the NN interaction. Continuing the discussion for the valence proton (particle 1 in Figure 3.1) the potential $V_{p p}\left(\boldsymbol{r}_{p}\right)$ in Eq. 3.23 now becomes $\hat{V}_{p p}\left(\boldsymbol{r}_{p}\right) P_{01}^{r}$. Substituting this into the general folding equation and acting with the operator $P_{01}^{r}$, which exchanges the spatial coordinates of the two nucleons i.e. $\boldsymbol{r}_{1} \rightarrow \boldsymbol{R}$ and $\boldsymbol{R} \rightarrow \boldsymbol{r}_{1}$, changes Eq. 3.23 to

$$
\begin{aligned}
& \left\langle\Psi_{I, M_{f}}\left(\boldsymbol{r}_{1}, \boldsymbol{r}_{2}\right)\left|\hat{V}_{p p}\left(\boldsymbol{r}_{p}\right) P_{01}^{r}\right| \Psi_{I, M_{i}}\left(\boldsymbol{r}_{1}, \boldsymbol{r}_{2}\right)\right\rangle \\
& =(-)^{3 j_{1}-j_{1}^{\prime}} \hat{I} \hat{j}_{1} \hat{j}_{1}^{\prime} \int d \boldsymbol{r}_{1} \sum_{\lambda_{1}^{\prime} \lambda_{1} m_{s 1}^{\prime} m_{s 1}} Y_{\ell_{1}^{\prime} \lambda_{1}^{\prime}}^{*}\left(\hat{\boldsymbol{r}}_{1}\right) \chi_{\frac{1}{2} m_{s 1}^{\prime}}^{*} u_{\ell_{1}^{\prime} j_{1}^{\prime}}\left(r_{1}\right) \hat{V}_{p p}\left(\boldsymbol{r}_{p}\right) Y_{\ell_{1} \lambda_{1}}(\hat{\boldsymbol{R}}) \chi_{\frac{1}{2} m_{s 1}} \\
& \quad \times u_{\ell_{1} j_{1}}(R)(-)^{\frac{1}{2}+m_{s 1}^{\prime}-\ell_{1}^{\prime}-\lambda_{1}} \sum_{k_{I} q_{I}} \hat{k}_{I}^{2} W\left(k_{I} j_{1} I j_{2} ; j_{1}^{\prime} I\right)\left(I M_{i} k_{I} q_{I} \mid I M_{f}\right) \sum_{k_{1} q_{1} k_{2} q_{2}}(-)^{k_{1}-k_{2}} \\
& \quad \times \hat{k}_{1}\left(\frac{1}{2} m_{s 1} \frac{1}{2}-m_{s 1}^{\prime} \mid k_{1} q_{1}\right)\left(\ell_{1}-\lambda_{1} \ell_{1}^{\prime} \lambda_{1}^{\prime} \mid k_{2} q_{2}\right)\left(k_{1} q_{1} k_{I} q_{I} \mid k_{2} q_{2}\right)\left\{\begin{array}{ccc}
j_{1} & j_{1}^{\prime} & k_{I} \\
\frac{1}{2} & \frac{1}{2} & k_{1} \\
\ell_{1} & \ell_{1}^{\prime} & k_{2}
\end{array}\right\} P_{01}^{r} .
\end{aligned}
$$


Remembering that in the calculation of the scattering amplitude this folded potential is placed between two distorted waves and the exchange operator will also act on the wavefunction to the right.

This folding equation can now be used to determine the folded spin-spin potentials from both the central exchange term $\hat{v}_{p p}^{\sigma \sigma}(r) \sigma_{0} \cdot \sigma_{1} P_{01}^{r}$ and the tensor exchange term $\hat{v}_{p p}^{t r}(r) \boldsymbol{S}_{01} P_{01}^{r}$ in the NN interaction.

\subsection{Central Exchange NN Interaction}

The folding of the central SNKE term $\hat{v}_{p p}^{\sigma \sigma}\left(r_{p}\right) \sigma_{0} \cdot \sigma_{1} P_{01}^{r}$ will be performed using the zero-range pseudo-potential from Eq. 4.20. Inserting this into Eq 5.1 yields

$$
\begin{aligned}
& \left\langle\Psi_{I, M_{f}}\left(\boldsymbol{r}_{1}, \boldsymbol{r}_{2}\right)\left|\hat{J}_{\sigma \sigma}(Q) \delta\left(\boldsymbol{r}_{p}\right) \boldsymbol{\sigma}_{0} \cdot \boldsymbol{\sigma}_{1} P_{01}^{r}\right| \Psi_{I, M_{i}}\left(\boldsymbol{r}_{1}, \boldsymbol{r}_{2}\right)\right\rangle \\
& \quad=(-)^{3 j_{1}-j_{1}^{\prime}-\ell_{1}^{\prime}} \hat{J}_{\sigma \sigma}(Q) \sqrt{\frac{6}{4 \pi}} \hat{I} \hat{j}_{1} \hat{j}_{1}^{\prime} \hat{\ell}_{1} \hat{\ell}_{1}^{\prime} u_{\ell_{1}^{\prime} j_{1}^{\prime}}(R) u_{\ell_{1} j_{1}}(R) \\
& \quad \times \sum_{k_{I} k} \hat{k_{I}} W\left(k_{I} j_{1} I j_{2} ; j_{1}^{\prime} I\right) \frac{(-)^{k}}{\hat{k}}\left(\ell_{1} 0 \ell_{1}^{\prime} 0 \mid k 0\right)\left\{\begin{array}{ccc}
j_{1} & j_{1}^{\prime} & k_{I} \\
\frac{1}{2} & \frac{1}{2} & 1 \\
\ell_{1} & \ell_{1}^{\prime} & k
\end{array}\right\}\left\langle I, M_{f}\left|\boldsymbol{S}_{i k}\right| I, M_{i}\right\rangle,
\end{aligned}
$$

where the full details of this folding are given in Appendix D.1.

\subsubsection{Spin-Spin Potentials for Central Exchange Term}

The spin-spin potentials allowed for the model of ${ }^{10} \mathrm{~B}$ used in this work are determined in the same way for the central exchange term as they were with the direct terms in the NN interaction. Two valence particles $(1,2)$ in the $1 \mathrm{p}_{\frac{3}{2}}$ have orbital angular momentum of $\ell_{1}=\ell_{1}^{\prime}=1$ and total angular momentum of $j_{1}=j_{1}^{\prime}=j_{2}=\frac{3}{2}$. Inserting these numbers into Eq. 5.2 along with the spin $I=3$ of ${ }^{10} \mathrm{~B}$ yields three different types of spin-spin interaction

$$
\begin{aligned}
& \hat{U}_{10}^{\sigma \sigma}(R)=-\frac{\hat{J}_{\sigma \sigma}(Q)}{\sqrt{3 \pi}} u_{1 \frac{3}{2}}\left(r_{1}\right)^{2} \boldsymbol{S}_{10} \\
& \hat{U}_{12}^{\sigma \sigma}(\boldsymbol{R})=-\frac{\hat{J}_{\sigma \sigma}(Q)}{5} \sqrt{\frac{2}{15 \pi}} u_{1 \frac{3}{2}}\left(r_{1}\right)^{2} \boldsymbol{S}_{12} \\
& \hat{U}_{32}^{\sigma \sigma}(\boldsymbol{R})=\frac{3}{5} \frac{\hat{J}_{\sigma \sigma}(Q)}{\sqrt{10 \pi}} u_{1 \frac{3}{2}}\left(r_{1}\right)^{2} \boldsymbol{S}_{32}
\end{aligned}
$$

where the hat denotes that these folded potentials are derived from the central $\left({ }^{\sigma \sigma}\right)$ exchange term in the NN interaction. These three spin-spin interactions are the same 
type as those obtained from the direct central term shown in Eqs. $3.31-3.33$.

\subsection{Tensor Exchange NN Interaction}

The contribution of the tensor SNKE term $\hat{v}_{p p}^{t r}\left(r_{p}\right) \boldsymbol{S}_{01} P_{01}^{r}$ to the folded spin-spin interactions will be approximated using the zero-range pseudo-potential from Eq. 4.27

$$
\begin{aligned}
& \hat{J}_{t r}(Q)\left[\hat{\boldsymbol{S}}_{01}\left(\boldsymbol{\sigma}_{0}, \boldsymbol{\sigma}_{1},-\imath \nabla_{\boldsymbol{r}_{p}}\right) \delta\left(\boldsymbol{r}_{p}\right)\right] P_{01}^{r} \\
& \quad=2 \sqrt{\frac{8 \pi}{5}} \hat{J}_{t r}(Q) \sum_{\mu}(-)^{\mu} \tau_{2-\mu}(\boldsymbol{s})\left[\mathscr{Y}_{2 \mu}\left(-\imath \nabla_{\boldsymbol{r}_{p}}\right) \delta\left(\boldsymbol{r}_{p}\right)\right] P_{01}^{r}
\end{aligned}
$$

The relationship between these two different forms of the pseudo-potential is analogous to that derived in Appendix C.2.1 for the direct tensor term operator. The second form, where the spin and radial dependence of the operator have been separated, is more suitable for manipulation in the folding model and will therefore be used for the evaluation of this term. The solid spherical harmonic $\mathscr{Y}_{2 \mu}\left(-\imath \nabla_{r_{p}}\right)$ described in [88] is related to the regular spherical harmonic by

$$
\mathscr{Y}_{2 \mu}(\boldsymbol{R})=R^{2} Y_{2 \mu}(\hat{\boldsymbol{R}}) .
$$

The solid spherical harmonic $\mathscr{Y}_{2 \mu}\left(-\imath \nabla_{r_{p}}\right)$ can be expressed in terms of the grad operator $\nabla_{\boldsymbol{r}_{p}}$ using the coupling rule for two spherical harmonics, Eq. A.16, rearranged to give

$$
Y_{\ell m}(\hat{\boldsymbol{R}})=\frac{\sqrt{4 \pi} \hat{\ell}}{\hat{\ell}_{1} \hat{\ell}_{2}\left(\ell_{1} 0 \ell_{2} 0 \mid \ell 0\right)} \sum_{m_{1} m_{2}}\left(\ell_{1} m_{1} \ell_{2} m_{2} \mid \ell m\right) Y_{\ell_{1} m_{1}}(\hat{\boldsymbol{R}}) Y_{\ell_{2} m_{2}}(\hat{\boldsymbol{R}})
$$

Taking $\ell_{1}=\ell_{2}=1$ and $\ell=2$ and multiplying both sides by $R^{2}$ gives

$$
\mathscr{Y}_{2 m}(\boldsymbol{R})=\frac{\sqrt{4 \pi} \sqrt{5}}{3(1010 \mid 20)} \sum_{m_{1} m_{2}}\left(1 m_{1} 1 m_{2} \mid 2 m\right) \mathscr{Y}_{1 m_{1}}(\boldsymbol{R}) \mathscr{Y}_{1 m_{2}}(\boldsymbol{R}) .
$$

Since $\boldsymbol{R}=\sum_{m_{1}} R_{m_{1}} e_{m_{1}}^{*}$ where $R_{m_{1}}=\sqrt{\frac{4 \pi}{3}} R Y_{1 m_{1}}(\hat{\boldsymbol{R}})$ then $\mathscr{Y}_{1 m_{1}}(\boldsymbol{R})=\sqrt{\frac{3}{4 \pi}}(R)_{m_{1}}$ and the solid spherical harmonic in Eq. 5.7 can be separated into

$$
\mathscr{Y}_{2 \mu}\left(-\imath \nabla_{\boldsymbol{r}_{N}}\right)=\frac{-\sqrt{5}}{\sqrt{4 \pi}} \sqrt{\frac{3}{2}} \sum_{\mu_{2} \mu_{3}}\left(1 \mu_{2} 1 \mu_{3} \mid 2 \mu\right) \nabla_{\mu_{2}} \nabla_{\mu_{3}}
$$

Expressing the solid spherical harmonic in this way will be useful for the folding of the tensor exchange term.

Now that the form of the tensor exchange term, Eq. 5.7, has been obtained, it can 
be substituted into the general exchange folding expression given in Eq. 5.1 to yield the following

$$
\begin{aligned}
& \left\langle\Psi_{I, M_{f}}\left(\boldsymbol{r}_{1}, \boldsymbol{r}_{2}\right)\left|2 \sqrt{\frac{8 \pi}{5}} \hat{J}_{t r}(Q) \sum_{\mu}(-)^{\mu} \tau_{2-\mu}(s)\left[\mathscr{Y}_{2 \mu}\left(-\imath \nabla_{\boldsymbol{r}_{p}}\right) \delta\left(\boldsymbol{r}_{p}\right)\right] P_{01}^{r}\right| \Psi_{I, M_{i}}\left(\boldsymbol{r}_{1}, \boldsymbol{r}_{2}\right)\right\rangle \\
& =(-)^{3 j_{1}-j_{1}^{\prime}} \hat{I} \hat{j}_{1} \hat{j}_{1}^{\prime} \int d \boldsymbol{r}_{1} \sum_{\lambda_{1}^{\prime} \lambda_{1} m_{s 1}^{\prime} m_{s 1}} Y_{\ell_{1}^{\prime} \lambda_{1}^{\prime}}^{*}\left(\hat{\boldsymbol{r}}_{1}\right) u_{\ell_{1}^{\prime} j_{1}^{\prime}}\left(r_{1}\right) \hat{J}_{t r}(Q) 2 \sqrt{\frac{8 \pi}{5}} \sum_{\mu \mu_{0} \mu_{1}}(-)^{\mu} \frac{\sqrt{3}}{2} \\
& \quad \times\left(1 \mu_{0} 1 \mu_{1} \mid 2-\mu\right) \tau_{1 \mu_{0}}\left(\boldsymbol{\sigma}_{0}\right) \sqrt{2}(-)^{1 / 2-m_{s 1}}\left(\frac{1}{2} m_{s 1} \frac{1}{2}-m_{s 1}^{\prime} \mid 1 \mu_{1}\right)\left[\mathscr{Y}_{2 \mu}\left(-\imath \nabla_{\boldsymbol{r}_{p}}\right) \delta\left(\boldsymbol{r}_{p}\right)\right] Y_{\ell_{1} \lambda_{1}}(\hat{\boldsymbol{R}}) \\
& \quad \times u_{\ell_{1} j_{1}}(R)(-)^{1 / 2+m_{s 1}^{\prime}-\ell_{1}^{\prime}-\lambda_{1}} \sum_{k_{I} q_{I}}{\hat{k_{I}}}^{2} W\left(k_{I} j_{1} I j_{2} ; j_{1}^{\prime} I\right)\left(I M_{i} k_{I} q_{I} \mid I M_{f}\right) \sum_{k_{1} q_{1} k_{2} q_{2}}(-)^{k_{1}-k_{2}} \\
& \quad \times \hat{k}_{1}\left(\frac{1}{2} m_{s 1} \frac{1}{2}-m_{s 1}^{\prime} \mid k_{1} q_{1}\right)\left(\ell_{1}-\lambda_{1} \ell_{1}^{\prime} \lambda_{1}^{\prime} \mid k_{2} q_{2}\right)\left(k_{1} q_{1} k_{I} q_{I} \mid k_{2} q_{2}\right)\left\{\begin{array}{ccc}
j_{1} & j_{1}^{\prime} & k_{I} \\
\frac{1}{2} & \frac{1}{2} & k_{1} \\
\ell_{1} & \ell_{1}^{\prime} & k_{2}
\end{array}\right\} P_{01}^{r},
\end{aligned}
$$

where the rank 2 spin operator $\tau_{2-\mu}(s)$ has been split using Eq. 3.36 and Eq. C.3 has been used for the target nucleon spin matrix element. For the following discussion, consideration must be taken that the operation of $P_{01}^{r}$ is not limited to the initial ground state valence nucleon wavefunction $\Psi_{I, M_{i}}\left(\boldsymbol{r}_{1}, \boldsymbol{r}_{2}\right)$. In the calculation of the scattering amplitude the initial distorted wave is also to the right of this operator (see Section 2.2). Therefore, when taking all the coordinate-dependent parts of Eq. 5.12, the distorted waves should also be included

$$
\chi_{\mu_{f}}^{*(-)}\left(\boldsymbol{R}, \boldsymbol{k}^{\prime}\right) \int d \boldsymbol{r}_{1} Y_{\ell_{1}^{\prime} \lambda_{1}^{\prime}}^{*}\left(\hat{\boldsymbol{r}}_{1}\right) u_{\ell_{1}^{\prime} j_{1}^{\prime}}\left(r_{1}\right)\left[\mathscr{Y}_{2 \mu}\left(-\imath \nabla_{\boldsymbol{r}_{p}}\right) \delta\left(\boldsymbol{r}_{p}\right)\right] Y_{\ell_{1} \lambda_{1}}(\hat{\boldsymbol{R}}) u_{\ell_{1} j_{1}}(R) \chi_{\mu_{i}}^{(+)}\left(\boldsymbol{r}_{1}, \boldsymbol{k}\right)
$$

where $P_{01}^{r} \chi_{\mu_{i}}^{(+)}(\boldsymbol{R}, \boldsymbol{k})=\chi_{\mu_{i}}^{(+)}\left(\boldsymbol{r}_{1}, \boldsymbol{k}\right)^{1}$. In order to take advantage of the integral properties of the delta function the coordinates can be changed to $\boldsymbol{r}_{1}=\boldsymbol{R}-\boldsymbol{r}_{p}$, which gives

$$
\begin{aligned}
\chi_{\mu_{f}}^{*(-)}\left(\boldsymbol{R}, \boldsymbol{k}^{\prime}\right) & \int d \boldsymbol{r}_{p} Y_{\ell_{1}^{\prime} \lambda_{1}^{\prime}}^{*}\left(\boldsymbol{R} \hat{-} \boldsymbol{r}_{1}\right) u_{\ell_{1}^{\prime} j_{1}^{\prime}}\left(\left|\boldsymbol{R}-\boldsymbol{r}_{p}\right|\right)\left[\mathscr{Y}_{2 \mu}\left(-\imath \nabla_{\boldsymbol{r}_{p}}\right) \delta\left(\boldsymbol{r}_{p}\right)\right] \\
& \times Y_{\ell_{1} \lambda_{1}}(\hat{\boldsymbol{R}}) u_{\ell_{1} j_{1}}(R) \chi_{\mu_{i}}^{(+)}\left(\boldsymbol{R}-\boldsymbol{r}_{p}, \boldsymbol{k}\right) .
\end{aligned}
$$

Integrating by parts or using the following relation twice, $\int d \boldsymbol{r} \phi \nabla \cdot \boldsymbol{A}=-\int d \boldsymbol{r}(\nabla \phi) \cdot \boldsymbol{A}$,

\footnotetext{
${ }^{1}$ For the central exchange term this consideration was not required. The integration in Eq D.2 changes $\chi_{\mu_{i}}^{(+)}\left(\boldsymbol{r}_{1}, \boldsymbol{k}\right)$ back to $\chi_{\mu_{i}}^{(+)}(\boldsymbol{R}, \boldsymbol{k})$
} 
which is true when either $\phi$ or $\boldsymbol{A}$ goes to zero as $r \rightarrow \infty$, leads to

$$
\begin{gathered}
\chi_{\mu_{f}}^{*(-)}\left(\boldsymbol{R}, \boldsymbol{k}^{\prime}\right) Y_{\ell_{1} \lambda_{1}}(\hat{\boldsymbol{R}}) u_{\ell_{1} j_{1}}(R) \int d \boldsymbol{r}_{p} \delta\left(\boldsymbol{r}_{p}\right) \mathscr{Y}_{2 \mu}\left(-\imath \nabla_{\boldsymbol{r}_{p}}\right) Y_{\ell_{1}^{\prime} \lambda_{1}^{\prime}}^{*}\left(\boldsymbol{R} \hat{-} \boldsymbol{r}_{1}\right) \\
\times u_{\ell_{1}^{\prime} j_{1}^{\prime}}\left(\left|\boldsymbol{R}-\boldsymbol{r}_{p}\right|\right) \chi_{\mu_{i}}^{(+)}\left(\boldsymbol{R}-\boldsymbol{r}_{p}, \boldsymbol{k}\right) .
\end{gathered}
$$

Making the substitution $\nabla_{\boldsymbol{r}_{p}}^{2} f\left(\boldsymbol{R}-\boldsymbol{r}_{p}\right)=\nabla_{\boldsymbol{R}}^{2} f\left(\boldsymbol{R}-\boldsymbol{r}_{p}\right)$ finally allows us to integrate using the properties of the delta function (Eq. D.2) resulting in

$$
\chi_{\mu_{f}}^{*(-)}\left(\boldsymbol{R}, \boldsymbol{k}^{\prime}\right) Y_{\ell_{1} \lambda_{1}}(\hat{\boldsymbol{R}}) u_{\ell_{1} j_{1}}(R) \mathscr{Y}_{2 \mu}\left(-\imath \nabla_{\boldsymbol{R}}\right) Y_{\ell_{1}^{\prime} \lambda_{1}^{\prime}}^{*}(\hat{\boldsymbol{R}}) u_{\ell_{1}^{\prime} j_{1}^{\prime}}(R) \chi_{\mu_{i}}^{(+)}(\boldsymbol{R}, \boldsymbol{k}),
$$

which can be split into three terms: A, where the solid spherical harmonic acts on the components of the valence nucleon wavefunction; $\mathrm{B}$, where it acts on the initial distorted wave; and $\mathrm{C}$, where a rank-one grad operator acts on both using Eq. 5.11

$$
\begin{aligned}
\chi_{\mu_{f}}^{*(-)}\left(\boldsymbol{R}, \boldsymbol{k}^{\prime}\right) Y_{\ell_{1} \lambda_{1}}(\hat{\boldsymbol{R}}) u_{\ell_{1} j_{1}}(R)\left\{\left[\mathscr{Y}_{2 \mu}\left(-\imath \nabla_{\boldsymbol{R}}\right) Y_{\ell_{1}^{\prime} \lambda_{1}^{\prime}}^{*}(\hat{\boldsymbol{R}}) u_{\ell_{1}^{\prime} j_{1}^{\prime}}(R)\right] \chi_{\mu_{i}}^{(+)}(\boldsymbol{R}, \boldsymbol{k})\right. \\
+\quad Y_{\ell_{1}^{\prime} \lambda_{1}^{\prime}}^{*}(\hat{\boldsymbol{R}}) u_{\ell_{1}^{\prime} j_{1}^{\prime}}(R)\left[\mathscr{Y}_{2 \mu}\left(-\imath \nabla_{\boldsymbol{R}}\right) \chi_{\mu_{i}}^{(+)}(\boldsymbol{R}, \boldsymbol{k})\right] \\
\left.-2 \frac{\hat{2}}{\sqrt{4 \pi}} \sqrt{\frac{3}{2}} \sum_{\mu_{1} \mu_{2}}\left(1 \mu_{2} 1 \mu_{3} \mid 2 \mu\right)\left[\nabla_{\mu_{2}} Y_{\ell_{1}^{\prime} \lambda_{1}^{\prime}}^{*}(\hat{\boldsymbol{R}}) u_{\ell_{1}^{\prime} j_{1}^{\prime}}(R)\right]\left[\nabla_{\mu_{2}^{\prime}} \chi_{\mu_{i}}^{(+)}(\boldsymbol{R}, \boldsymbol{k})\right]\right\},
\end{aligned}
$$

substituting this back into Eq. 5.12 and using the orthogonality of the C-G coefficients given in Eq. A.1 leads to

$$
\begin{aligned}
& \left\langle\Psi_{I, M_{f}}\left(\boldsymbol{r}_{1}, \boldsymbol{r}_{2}\right)\left|\hat{J}_{t r}(Q) 2 \sqrt{\frac{8 \pi}{5}} \sum_{\mu}(-)^{\mu} \tau_{2-\mu}(\boldsymbol{s})\left[\mathscr{Y}_{2 \mu}\left(-\imath \nabla_{\boldsymbol{r}_{p}}\right) \delta\left(\boldsymbol{r}_{p}\right)\right]\right| \Psi_{I, M_{i}}\left(\boldsymbol{R}, \boldsymbol{r}_{2}\right)\right\rangle \chi_{\mu_{i}}^{(+)}\left(\boldsymbol{r}_{1}, \boldsymbol{k}\right) \\
& =(-)^{3 j_{1}-j_{1}^{\prime}-\ell_{1}^{\prime}} \hat{J}_{t r}(Q) 3 \sqrt{2} \sqrt{\frac{8 \pi}{5}} \hat{I} \hat{j}_{1} \hat{j}_{1}^{\prime} \sum_{\mu \mu_{0} \mu_{1}}(-)^{\mu+\mu_{1}}\left(1 \mu_{0} 1 \mu_{1} \mid 2-\mu\right) \tau_{1 \mu_{0}}\left(\boldsymbol{\sigma}_{0}\right) \sum_{\lambda_{1}^{\prime} \lambda_{1}} \\
& \times\left\{\left[\mathscr{Y}_{2 \mu}\left(-\imath \nabla_{\boldsymbol{R}}\right) Y_{\ell_{1}^{\prime} \lambda_{1}^{\prime}}^{*}(\hat{\boldsymbol{R}}) u_{\ell_{1}^{\prime} j_{1}^{\prime}}(R)\right] \chi_{\mu_{i}}^{(+)}(\boldsymbol{R}, \boldsymbol{k}) \quad A\right. \\
& +Y_{\ell_{1}^{\prime} \lambda_{1}^{\prime}}^{*}(\hat{\boldsymbol{R}}) u_{\ell_{1}^{\prime} j_{1}^{\prime}}(R)\left[\mathscr{Y}_{2 \mu}\left(-\imath \nabla_{\boldsymbol{R}}\right) \chi_{\mu_{i}}^{(+)}(\boldsymbol{R}, k)\right] \quad B \\
& \left.-2 \frac{\hat{2}}{\sqrt{4 \pi}} \sqrt{\frac{3}{2}} \sum_{\mu_{1} \mu_{2}}\left(1 \mu_{2} 1 \mu_{3} \mid 2 \mu\right)\left[\nabla_{\mu_{2}} Y_{\ell_{1}^{\prime} \lambda_{1}^{\prime}}^{*}(\hat{\boldsymbol{R}}) u_{\ell_{1}^{\prime} j_{1}^{\prime}}(R)\right]\left[\nabla_{\mu_{2}^{\prime}} \chi_{\mu_{i}}^{(+)}(\boldsymbol{R}, \boldsymbol{k})\right]\right\} C \\
& \times(-)^{-\lambda_{1}} Y_{\ell_{1} \lambda_{1}}(\hat{\boldsymbol{R}}) u_{\ell_{1} j_{1}}(R) \sum_{k_{I} q_{I}}{\hat{k_{I}}}^{2} W\left(k_{I} j_{1} I j_{2} ; j_{1}^{\prime} I\right) \\
& \times\left(I M_{i} k_{I} q_{I} \mid I M_{f}\right) \sum_{k_{2} q_{2}}(-)^{-k_{2}}\left(\ell_{1}-\lambda_{1} \ell_{1}^{\prime} \lambda_{1}^{\prime} \mid k_{2} q_{2}\right)\left(1-\mu_{1} k_{I} q_{I} \mid k_{2} q_{2}\right)\left\{\begin{array}{ccc}
j_{1} & j_{1}^{\prime} & k_{I} \\
\frac{1}{2} & \frac{1}{2} & 1 \\
\ell_{1} & \ell_{1}^{\prime} & k_{2}
\end{array}\right\},
\end{aligned}
$$


where the incident distorted wave $\chi_{\mu_{i}}^{(+)}(\boldsymbol{R}, \boldsymbol{k})$ has been left in the equation for clarity. In a symmetry with the direct tensor folded potential there are three terms to evaluate which will be dealt with separately.

\subsubsection{Spin-Spin Potentials for Tensor Exchange Term A}

The folding, using Eq. 5.18, of the term where the solid harmonic acts only on the components of the final valence nucleon wavefunction, $\left[\mathscr{Y}_{2 \mu}\left(-\imath \nabla_{\boldsymbol{R}}\right) Y_{\ell_{1}^{\prime} \lambda_{1}^{\prime}}^{*}(\hat{\boldsymbol{R}}) u_{\ell_{1}^{\prime} j_{1}^{\prime}}(R)\right]$ and not on the distorted waves gives

\section{Term A}

$$
\begin{aligned}
& =(-)^{3 j_{1}-j_{1}^{\prime}-\ell_{1}^{\prime}} \hat{J}_{t r}(Q) 3 \sqrt{2} \sqrt{\frac{8 \pi}{5}} \hat{I} \hat{j}_{1} \hat{j}_{1}^{\prime} \sum_{\mu \mu_{0} \mu_{1}}(-)^{\mu+\mu_{1}}\left(1 \mu_{0} 1 \mu_{1} \mid 2-\mu\right) \tau_{1 \mu_{0}}\left(\boldsymbol{\sigma}_{0}\right) \\
& \times \sum_{\lambda_{1}^{\prime} \lambda_{1}}\left[\mathscr{Y}_{2 \mu}\left(-\imath \nabla_{\boldsymbol{R}}\right) Y_{\ell_{1}^{\prime} \lambda_{1}^{\prime}}^{*}(\hat{\boldsymbol{R}}) u_{\ell_{1}^{\prime} j_{1}^{\prime}}(R)\right](-)^{-\lambda_{1}} Y_{\ell_{1} \lambda_{1}}(\hat{\boldsymbol{R}}) u_{\ell_{1} j_{1}}(R) \\
& \times \sum_{k_{I} q_{I}}{\hat{k_{I}}}^{2} W\left(k_{I} j_{1} I j_{2} ; j_{1}^{\prime} I\right)\left(I M_{i} k_{I} q_{I} \mid I M_{f}\right) \sum_{k_{2} q_{2}}(-)^{-k_{2}} \\
& \times\left(\ell_{1}-\lambda_{1} \ell_{1}^{\prime} \lambda_{1}^{\prime} \mid k_{2} q_{2}\right)\left(1-\mu_{1} k_{I} q_{I} \mid k_{2} q_{2}\right)\left\{\begin{array}{ccc}
j_{1} & j_{1}^{\prime} & k_{I} \\
\frac{1}{2} & \frac{1}{2} & 1 \\
\ell_{1} & \ell_{1}^{\prime} & k_{2}
\end{array}\right\}
\end{aligned}
$$

as the distorted wave are unaffected by this term they have not been included in this equation. Using the angular momentum coupling techniques detailed in Appendix D.3, Eq. 5.19 becomes

\section{Term A}

$$
\begin{aligned}
& =(-)^{1+3 j_{1}-j_{1}^{\prime}-\ell_{1}^{\prime}} \hat{J}_{t r}(Q) \frac{6 \sqrt{5}}{\sqrt{4 \pi}} \hat{I} \hat{\ell}_{1} \hat{j}_{1} \hat{j}_{1}^{\prime} u_{\ell_{1} j_{1}}(R) \sum_{k_{I} k_{2} L C}\left[\hat{\mathscr{O}}_{L, \ell_{1}^{\prime}} u_{\ell_{1}^{\prime} j_{1}^{\prime}}(R)\right] \\
& \times(-)^{-k_{2}-k_{I}-C} \frac{\hat{L}^{2} \hat{k}_{2}^{2}}{\hat{C}}\left(20 L 0 \mid \ell_{1}^{\prime} 0\right)\left(\ell_{1} 0 L 0 \mid C 0\right) \hat{k_{I}} W\left(k_{I} j_{1} I j_{2} ; j_{1}^{\prime} I\right) \\
& \times W\left(2 \ell_{1}^{\prime} C \ell_{1} ; L k_{2}\right) W\left(11 C k_{2} ; 2 i\right)\left\{\begin{array}{ccc}
j_{1} & j_{1}^{\prime} & k_{I} \\
\frac{1}{2} & \frac{1}{2} & 1 \\
\ell_{1} & \ell_{1}^{\prime} & k_{2}
\end{array}\right\}\left\langle I, M_{f}\left|S_{k_{I} C}\right| I, M_{i}\right\rangle .
\end{aligned}
$$

The differential operator $\hat{\mathscr{O}}_{L \ell_{1}^{\prime}}$ is taken from [89] and described in Appendix D.2. This spin-spin interaction is of the same form, $U_{k_{I} k}(\boldsymbol{R})=F_{k_{I} k}(R) \boldsymbol{S}_{k_{I} k}$, as all the other folded potentials derived previously. The spin-spin amplitude can therefore be calculated in the same way, using the formalism detailed in Chapter 2. 
Using the angular momentum values from the simplified model of ${ }^{10} \mathrm{~B}$ and performing the sums in Eq. 5.20 in exactly the same way as the other folded potentials yields the allowed spin-spin terms

$$
\begin{aligned}
\hat{U}_{10}^{t r A}(R) & =\frac{-2 \hat{J}_{t r}(Q)}{5 \sqrt{3 \pi}} u_{1 \frac{3}{2}}\left(r_{1}\right)\left[\hat{\mathscr{O}}_{11} u_{1 \frac{3}{2}}(R)\right] \boldsymbol{S}_{10} \\
\hat{U}_{12}^{t r A}(\boldsymbol{R}) & =\frac{-\hat{J}_{t r}(Q)}{25 \sqrt{30 \pi}} u_{1 \frac{3}{2}}\left(r_{1}\right)\left[13 \hat{\mathscr{O}}_{11}+27 \hat{\mathscr{O}}_{31} u_{1 \frac{3}{2}}(R)\right] \boldsymbol{S}_{12} \\
\hat{U}_{22}^{t r A}(\boldsymbol{R}) & =\frac{9 \hat{J}_{t r}(Q)}{25 \sqrt{10 \pi}} u_{1 \frac{3}{2}}\left(r_{1}\right)\left[\hat{\mathscr{O}}_{11}-\hat{\mathscr{O}}_{31} u_{1 \frac{3}{2}}(R)\right] \boldsymbol{S}_{22} \\
\hat{U}_{32}^{t r A}(\boldsymbol{R}) & =\frac{-3 \hat{J}_{t r}(Q)}{175 \sqrt{10 \pi}} u_{1 \frac{3}{2}}\left(r_{1}\right)\left[7 \hat{\mathscr{O}}_{11}+3 \hat{\mathscr{O}}_{31} u_{1 \frac{3}{2}}(R)\right] \boldsymbol{S}_{32}, \\
\hat{U}_{34}^{t r A}(\boldsymbol{R}) & =\frac{3 \sqrt{6} \hat{J}_{t r}(Q)}{35 \sqrt{\pi}} u_{1 \frac{3}{2}}\left(r_{1}\right)\left[\hat{\mathscr{O}}_{31} u_{1 \frac{3}{2}}(R)\right] \boldsymbol{S}_{34} .
\end{aligned}
$$

The hat denotes that these spin-spin potentials are from the folding of an exchange term and the superscript ${ }^{\operatorname{tr} A}$ indicates which tensor term was folded to obtain these interactions.

For the first time, a spin-spin interaction is obtained that has even rank in $\boldsymbol{I}\left(k_{I}=2\right)$. The nonlocal nature of the tensor exchange term in the NN interaction means it is no longer straightforward to check the parity and time-reversal invariance of the resulting folded potentials. While the whole term (including parts A, B and C) must be invariant, the individual terms need not be. This issue will be dealt with in more detail in the exchange folding checks (Section 5.5).

\subsubsection{Spin-Spin Potentials for Tensor Exchange Term B}

The term in Eq 5.18 in which the solid spherical harmonic acts on the incident distorted wave alone $Y_{\ell_{1}^{\prime} \lambda_{1}^{\prime}}^{*}(\hat{\boldsymbol{R}}) u_{\ell_{1}^{\prime} j_{1}^{\prime}}(R)\left[\mathscr{Y}_{2 \mu}\left(-\imath \nabla_{\boldsymbol{R}}\right) \chi_{\mu_{i}}^{(+)}(\boldsymbol{R}, \boldsymbol{k})\right]$, with the valence nucleon wavefunction unaffected, yields the following folded potential

\section{Term B}

$$
\begin{aligned}
& =(-)^{3 j_{1}-j_{1}^{\prime}-\ell_{1}^{\prime}} \hat{J}_{t r}(Q) 3 \sqrt{2} \sqrt{\frac{8 \pi}{5}} \hat{I} \hat{j}_{1} \hat{j}_{1}^{\prime} \sum_{\mu \mu_{0} \mu_{1}}(-)^{\mu+\mu_{1}}\left(1 \mu_{0} 1 \mu_{1} \mid 2-\mu\right) \tau_{1 \mu_{0}}\left(\boldsymbol{\sigma}_{0}\right) \\
& \times \sum_{\lambda_{1}^{\prime} \lambda_{1}} Y_{\ell_{1}^{\prime} \lambda_{1}^{\prime}}^{*}(\hat{\boldsymbol{R}}) u_{\ell_{1}^{\prime} j_{1}^{\prime}}(R)(-)^{-\lambda_{1}} Y_{\ell_{1} \lambda_{1}}(\hat{\boldsymbol{R}}) u_{\ell_{1} j_{1}}(R) \sum_{k_{I} q_{I}}{\hat{k_{I}}}^{2} W\left(k_{I} j_{1} I j_{2} ; j_{1}^{\prime} I\right) \\
& \times\left(I M_{i} k_{I} q_{I} \mid I M_{f}\right) \sum_{k_{2} q_{2}}(-)^{-k_{2}}\left(\ell_{1}-\lambda_{1} \ell_{1}^{\prime} \lambda_{1}^{\prime} \mid k_{2} q_{2}\right)\left(1-\mu_{1} k_{I} q_{I} \mid k_{2} q_{2}\right)\left\{\begin{array}{ccc}
j_{1} & j_{1}^{\prime} & k_{I} \\
\frac{1}{2} & \frac{1}{2} & 1 \\
\ell_{1} & \ell_{1}^{\prime} & k_{2}
\end{array}\right\} \\
& \times\left[\mathscr{Y}_{2 \mu}^{\prime}\left(-\imath \nabla_{\boldsymbol{R}}\right) \chi_{\mu_{i}}^{(+)}\left(\boldsymbol{R}, \boldsymbol{k}_{i}\right)\right] .
\end{aligned}
$$


Using the method detailed in Appendix D.4 this reduces to

\section{Term B}

$$
\begin{aligned}
& =(-)^{1+3 j_{1}-j_{1}^{\prime}-\ell_{1}^{\prime}} \hat{J}_{t r}(Q) 2 \hat{I} \hat{j}_{1} \hat{j}_{1}^{\prime} \hat{\ell}_{1} \hat{\ell}_{1}^{\prime} u_{\ell_{1}^{\prime} j_{1}^{\prime}}(R) u_{\ell_{1} j_{1}}(R) \\
& \times \sum_{k_{I} C}(-)^{k_{I}-C}\left(\ell_{1} 0 \ell_{1}^{\prime} 0 \mid C 0\right) \hat{k}_{I} W\left(k_{I} j_{1} I j_{2} ; j_{1}^{\prime} I\right)\left\{\begin{array}{ccc}
j_{1} & j_{1}^{\prime} & k_{I} \\
\frac{1}{2} & \frac{1}{2} & 1 \\
\ell_{1} & \ell_{1}^{\prime} & C
\end{array}\right\} \\
& \times \sum_{\Gamma q_{I} \mu_{0} \mu_{1} \mu}(-1)^{\mu_{1}}\left(C \Gamma k_{I} q_{I} \mid 1 \mu_{1}\right) Y_{C \Gamma}(\hat{\boldsymbol{R}})\left\langle I, M_{\hat{j}}\left|\tau_{k_{I} q_{I}}(\boldsymbol{I})\right| I, M_{i}\right\rangle\left(1 \ell_{\ell_{0}} 2 \mu \mid 1-\mu_{1}\right) \tau_{1 \mu_{0}}\left(\boldsymbol{\sigma}_{0}\right) \\
& \times\left[\mathscr{Y}_{2 \mu}\left(-\imath \nabla_{\boldsymbol{R}}\right) \chi_{\mu_{i}}^{(+)}(\boldsymbol{R}, \boldsymbol{k})\right],
\end{aligned}
$$

while this appears complicated it is essentially a product of two different parts, a form factor that depends only on $R$

$$
\begin{aligned}
\hat{F}_{k_{I} k}^{t r B}(R) & =(-)^{1+3 j_{1}-j_{1}^{\prime}-\ell_{1}^{\prime}} \hat{J}_{t r}(Q) 2 \sqrt{4 \pi} \hat{I} \hat{j}_{1} \hat{j}_{1}^{\prime} \hat{\ell}_{1} \hat{\ell}_{1}^{\prime} u_{\ell_{1}^{\prime} j_{1}^{\prime}}(R) u_{\ell_{1} j_{1}}(R) \\
& \times(-)^{k_{I}-k}\left(\ell_{1} 0 \ell_{1}^{\prime} 0 \mid k 0\right) \hat{k_{I}} W\left(k_{I} j_{1} I j_{2} ; j_{1}^{\prime} I\right)\left\{\begin{array}{ccc}
j_{1} & j_{1}^{\prime} & k_{I} \\
\frac{1}{2} & \frac{1}{2} & 1 \\
\ell_{1} & \ell_{1}^{\prime} & k
\end{array}\right\},
\end{aligned}
$$

and an operator $\left[Y_{k}(\hat{\boldsymbol{R}}) \times \tau_{k_{I}}(\boldsymbol{I})\right] \cdot\left[\tau_{1}\left(\boldsymbol{\sigma}_{0}\right) \times \mathscr{Y}_{2}\left(-\imath \nabla_{\boldsymbol{R}}\right)\right]$. Therefore, Eq. 5.27 can be written more clearly as

$$
\hat{U}_{k_{I} k}^{t r B}(\boldsymbol{R}) \chi_{\mu_{i}}^{(+)}(\boldsymbol{R}, \boldsymbol{k})=\sum_{k_{I} k} \hat{F}_{k_{I} k}^{t r B}(R)\left[Y_{k}(\hat{\boldsymbol{R}}) \times \tau_{k_{I}}(\boldsymbol{I})\right] \cdot\left[\tau_{1}\left(\boldsymbol{\sigma}_{0}\right) \times \mathscr{Y}_{2}\left(-\imath \nabla_{\boldsymbol{R}}\right)\right] \chi_{\mu_{i}}^{(+)}(\boldsymbol{R}, \boldsymbol{k}),
$$

where the folded potentials from this tensor exchange term will be denoted by $\hat{U}_{k_{I} k}^{\operatorname{tr} B}(\boldsymbol{R})$. They are different from those derived previously because, instead of the operator $\boldsymbol{S}_{k_{I} k}$, these spin-spin interactions are proportional to $\left[Y_{k}(\hat{\boldsymbol{R}}) \times \tau_{k_{I}}(\boldsymbol{I})\right] \cdot\left[\tau_{1}\left(\boldsymbol{\sigma}_{0}\right) \times \mathscr{Y}_{2}\left(-\imath \nabla_{\boldsymbol{R}}\right)\right]$. Performing the sums in Eq. 5.27 and using the angular momentum values from the simplified model of ${ }^{10} \mathrm{~B}$ gives the allowed spin-spin terms

$$
\begin{aligned}
& \hat{U}_{10}^{t r B}(R)=-\frac{2 \sqrt{2}}{3} \hat{J}_{t r}(Q) u_{1 \frac{3}{2}}(R)^{2}\left[Y_{0}(\hat{\boldsymbol{R}}) \times \tau_{1}(\boldsymbol{I})\right] \cdot\left[\tau_{1}\left(\boldsymbol{\sigma}_{0}\right) \times \mathscr{Y}_{2 \mu}\left(-\imath \nabla_{\boldsymbol{R}}\right)\right], \\
& \hat{U}_{12}^{t r B}(\boldsymbol{R})=-\frac{4}{15} \hat{J}_{t r}(Q) u_{1 \frac{3}{2}}(R)^{2}\left[Y_{2}(\hat{\boldsymbol{R}}) \times \tau_{1}(\boldsymbol{I})\right] \cdot\left[\tau_{1}\left(\boldsymbol{\sigma}_{0}\right) \times \mathscr{Y}_{2 \mu}\left(-\imath \nabla_{\boldsymbol{R}}\right)\right], \\
& \hat{U}_{32}^{\operatorname{tr} B}(\boldsymbol{R})=\frac{\sqrt{12}}{5} \hat{J}_{t r}(Q) u_{1 \frac{3}{2}}(R)^{2}\left[Y_{2}(\hat{\boldsymbol{R}}) \times \tau_{3}(\boldsymbol{I})\right] \cdot\left[\tau_{1}\left(\boldsymbol{\sigma}_{0}\right) \times \mathscr{Y}_{2 \mu}\left(-\imath \nabla_{\boldsymbol{R}}\right)\right] .
\end{aligned}
$$


The solid spherical harmonics in these equations act on the initial distorted wavefunction. This means the spin-spin amplitude given in Eq. 2.27 cannot be used for this spin-spin interaction. The modification of the DWBA scattering amplitude for these potential terms will be discussed in Section 5.4.1.

\subsubsection{Spin-Spin Potentials for Tensor Exchange Term C}

The folding of the third tensor exchange term in Eq 5.18, which acts one grad on the components of the valence nucleon wavefunction and one grad on the distorted wave

$$
-2 \frac{\hat{2}}{\sqrt{4 \pi}} \sqrt{\frac{3}{2}} \sum_{\mu_{1} \mu_{2}}\left(1 \mu_{2} 1 \mu_{3} \mid 2 \mu\right)\left[\nabla_{\mu_{2}} Y_{\ell_{1}^{\prime} \lambda_{1}^{\prime}}^{*}(\hat{\boldsymbol{R}}) u_{\ell_{1}^{\prime} j_{1}^{\prime}}(R)\right]\left[\nabla_{\mu_{3}} \chi_{\mu_{i}}^{(+)}(\boldsymbol{R}, \boldsymbol{k})\right]
$$

can be folded using Eq. 5.18 to give

Term C

$$
\begin{aligned}
& =(-)^{3 j_{1}-j_{1}^{\prime}-\ell_{1}^{\prime}} \hat{J}_{t r}(Q) 3 \sqrt{2} \sqrt{\frac{8 \pi}{5}} \hat{I}_{j_{1}} \hat{j}_{1}^{\prime} \sum_{\mu \mu_{0} \mu_{1}}(-)^{\mu+\mu_{1}}\left(1 \mu_{0} 1 \mu_{1} \mid 2-\mu\right) \tau_{1 \mu_{0}}\left(\boldsymbol{\sigma}_{0}\right) \\
& \times \sum_{\lambda_{1}^{\prime} \lambda_{1}}(-2) \frac{\hat{2}}{\sqrt{4 \pi}} \sqrt{\frac{3}{2}} \sum_{\mu_{1} \mu_{2}}\left(1 \mu_{2} 1 \mu_{3} \mid 2 \mu\right)\left[\nabla_{\mu_{2}} Y_{\ell_{1}^{\prime} \lambda_{1}^{\prime}}^{*}(\hat{\boldsymbol{R}}) u_{\ell_{1}^{\prime} j_{1}^{\prime}}(R)\right](-)^{-\lambda_{1}} Y_{\ell_{1} \lambda_{1}}(\hat{\boldsymbol{R}}) \\
& \times u_{\ell_{1} j_{1}}(R) \sum_{k_{I} q_{I}} \hat{k}_{I}^{2} W\left(k_{I} j_{1} I j_{2} ; j_{1}^{\prime} I\right)\left(I M_{i} k_{I} q_{I} \mid I M_{f}\right) \sum_{k_{2} q_{2}}(-)^{-k_{2}} \\
& \times\left(\ell_{1}-\lambda_{1} \ell_{1}^{\prime} \lambda_{1}^{\prime} \mid k_{2} q_{2}\right)\left(1-\mu_{1} k_{I} q_{I} \mid k_{2} q_{2}\right)\left\{\begin{array}{ccc}
j_{1} & j_{1}^{\prime} & k_{I} \\
\frac{1}{2} & \frac{1}{2} & 1 \\
\ell_{1} & \ell_{1}^{\prime} & k_{2}
\end{array}\right\}\left[\nabla_{\mu_{3}} \chi_{\mu_{i}}^{(+)}(R, k)\right] .
\end{aligned}
$$

The angular momentum coupling techniques detailed in Appendix D.5 can be used to reduce this to

Term C

$$
\begin{aligned}
& =(-)^{3 j_{1}-j_{1}^{\prime}} \hat{J}_{t r}(Q) 30 \sqrt{\frac{6}{4 \pi}} \hat{I} \hat{j}_{1} \hat{j}_{1}^{\prime} \hat{\ell}_{1} \hat{\ell}_{1}^{\prime} u_{\ell_{1} j_{1}}(R) \sum_{k_{I} k_{2} L C E}\left[\hat{g}_{L \ell_{1}^{\prime}} u_{\ell_{1}^{\prime} j_{1}^{\prime}}(R)\right] \\
& \times(-)^{k_{I}-L} \hat{L} \hat{k}_{2}^{2}\left(\ell_{1}^{\prime} 010 \mid L 0\right)\left(\ell_{1} 0 L 0 \mid C 0\right) W\left(1 \ell_{1}^{\prime} C \ell_{1} ; L k_{2}\right) \hat{k}_{I} W\left(k_{I} j_{1} I j_{2} ; j_{1}^{\prime} I\right) \\
& \times\left\{\begin{array}{ccc}
j_{1} & j_{1}^{\prime} & k_{I} \\
\frac{1}{2} & \frac{1}{2} & 1 \\
\ell_{1} & \ell_{1}^{\prime} & k_{2}
\end{array}\right\}\left\{\begin{array}{ccc}
1 & C & E \\
1 & k_{2} & k_{I} \\
2 & 1 & 1
\end{array}\right\} \sum_{\Gamma q_{I} \mu_{0} \mu_{3}}(-1)^{\epsilon}\left(C \Gamma 1 \mu_{0} \mid E \epsilon\right) Y_{C \Gamma}(\hat{\boldsymbol{R}}) \tau_{1 \mu_{0}}\left(\boldsymbol{\sigma}_{0}\right) \\
& \times\left(k_{I} q_{I} 1 \mu_{3} \mid E-\epsilon\right)\left\langle I, M_{f}\left|\tau_{k_{I} q_{I}}(\boldsymbol{I})\right| I, M_{i}\right\rangle\left[\nabla_{\mu_{3}} \chi_{\mu_{i}}^{(+)}(\boldsymbol{R}, \boldsymbol{k})\right] .
\end{aligned}
$$


The differential operator $\hat{g}_{L \ell_{1}^{\prime}}$, which acts on the radial function $u_{\ell_{1}^{\prime} j_{1}^{\prime}}(R)$, from the final valence nucleon wavefunction is also taken from [89] and is defined in Appendix D.2. Eq. 5.35 can also be written more clearly as

$$
\hat{U}_{k_{I} k E}^{t r C} \chi_{\mu_{i}}^{(+)}(\boldsymbol{R}, \boldsymbol{k})=\sum_{k_{I} k E} \hat{F}_{k_{I} k E}^{t r C}(R)\left[Y_{k}(\hat{\boldsymbol{R}}) \times \tau_{1}\left(\boldsymbol{\sigma}_{0}\right)\right]_{E \epsilon}\left[\tau_{k_{I}}(\boldsymbol{I}) \times \nabla\right]_{E-\epsilon} \chi_{\mu_{i}}^{(+)}(\boldsymbol{R}, \boldsymbol{k})
$$

where the form factor $\hat{F}_{k_{I} k E}^{t r C}(R)$ is given by

$$
\begin{aligned}
\hat{F}_{k_{I} k E}^{t r C}(R) & =(-)^{1+3 j_{1}-j_{1}^{\prime}+\ell_{1}+\ell_{1}^{\prime}} \hat{J}_{t r}(Q) 30 \sqrt{\frac{6}{4 \pi}} \hat{I} \hat{j}_{1} \hat{j}_{1}^{\prime} \hat{\ell}_{1} u_{\ell_{1} j_{1}}(R) \sum_{k_{2} L}\left[\hat{g}_{L \ell_{1}^{\prime}} u_{\ell_{1}^{\prime} j_{1}^{\prime}}(R)\right] \\
& \times(-)^{k-k_{I}-L} \hat{L} \hat{k}_{2}^{2}\left(\ell_{1}^{\prime} 010 \mid L 0\right)\left(\ell_{1}\right) L(0 \mid k 0) W\left(1 \ell_{1}^{\prime} k \ell_{1} ; L k_{2}\right) \\
& \times \hat{k}_{I} W\left(k_{I} j_{1} I j_{2} ; j_{1}^{\prime} I\right)\left\{\begin{array}{ccc}
j_{1} & j_{1}^{\prime} & k_{I} \\
\frac{1}{2} & \frac{1}{2} & 1 \\
\ell_{1} & \ell_{1}^{\prime} & k_{2}
\end{array}\right\}\left\{\begin{array}{ccc}
1 & k & E \\
1 & k_{2} & k_{I} \\
2 & 1 & 1
\end{array}\right\},
\end{aligned}
$$

and the folded potentials from this tensor exchange term will be denoted by $\hat{U}_{k_{I} k E}^{t r C}$ where the extra subscript $E$ represents the coupling between the operators $\left[Y_{k}(\hat{\boldsymbol{R}}) \times \tau_{1}\left(\boldsymbol{\sigma}_{0}\right)\right]$ and $\left[\tau_{k_{I}}(\boldsymbol{I}) \times \nabla\right]$. Performing the sums in Eq. 5.35 with the angular momentum values from the simplified model of ${ }^{10} \mathrm{~B}$ leads to the following allowed spin-spin terms

$$
\begin{aligned}
& \hat{U}_{011}^{t r C}(\boldsymbol{R})=\frac{5 \hat{J}_{t r}(Q)}{6 \sqrt{6 \pi}} u_{1 \frac{3}{2}}(R)\left[\hat{g}_{01}-\hat{g}_{21}\right] u_{1 \frac{3}{2}}(R)\left[Y_{1}(\hat{\boldsymbol{R}}) \times \tau_{1}\left(\boldsymbol{\sigma}_{0}\right)\right]_{1 \epsilon}\left[\tau_{0}(\boldsymbol{I}) \times \nabla\right]_{1-\epsilon} \\
& \hat{U}_{110}^{t r C}(\boldsymbol{R})=\frac{-\hat{J}_{t r}(Q)}{5 \sqrt{3 \pi}} u_{1 \frac{3}{2}}(R)\left[5 \hat{g}_{01}+11 \hat{g}_{21}\right] u_{1 \frac{3}{2}}(R)\left[Y_{1}(\hat{\boldsymbol{R}}) \times \tau_{1}\left(\boldsymbol{\sigma}_{0}\right)\right]_{0}\left[\tau_{1}(\boldsymbol{I}) \times \nabla\right]_{0} \\
& \left.\hat{U}_{111}^{t r C}(\boldsymbol{R})=\frac{\hat{J}_{t r}(Q)}{15 \sqrt{3 \pi}} u_{1 \frac{3}{2}}(R)\left[5 \hat{g}_{01}+16 \hat{g}_{21}\right] u_{1} \frac{3}{2}(R)\left[Y_{1}(\hat{\boldsymbol{R}}) \times \tau_{1}\left(\boldsymbol{\sigma}_{0}\right)\right]_{1 \epsilon}\left[\tau_{1}(\boldsymbol{I}) \times \nabla\right)\right]_{1-\epsilon}, \\
& \hat{U}_{112}^{t r C}(\boldsymbol{R})=\frac{\hat{J}_{t r}(Q)}{25 \sqrt{3 \pi}} u_{1 \frac{3}{2}}(R)\left[10 \hat{g}_{01}-3 \hat{g}_{21}\right] u_{1 \frac{3}{2}}(R)\left[Y_{1}(\hat{\boldsymbol{R}}) \times \tau_{1}\left(\boldsymbol{\sigma}_{0}\right)\right]_{2 \epsilon}\left[\tau_{1}(\boldsymbol{I}) \times \nabla\right]_{2-\epsilon} \\
& \hat{U}_{132}^{t r C}(\boldsymbol{R})=\frac{-\sqrt{2} \hat{J}_{t r}(Q)}{25 \sqrt{\pi}} u_{1 \frac{3}{2}}(R)\left[\hat{g}_{21} u_{1 \frac{3}{2}}(R)\right]\left[Y_{3}(\hat{\boldsymbol{R}}) \times \tau_{1}\left(\boldsymbol{\sigma}_{0}\right)\right]_{2 \epsilon}\left[\tau_{1}(\boldsymbol{I}) \times \nabla\right]_{2-\epsilon} \\
& \left.\hat{U}_{211}^{t r C}(\boldsymbol{R})=\frac{-2 \hat{J}_{t r}(Q)}{3 \sqrt{5 \pi}} u_{1 \frac{3}{2}}(R)\left[\hat{g}_{01}-\hat{g}_{21}\right] u_{1 \frac{3}{2}}(R)\left[Y_{1}(\hat{\boldsymbol{R}}) \times \tau_{1}\left(\boldsymbol{\sigma}_{0}\right)\right]_{1 \epsilon}\left[\tau_{2}(\boldsymbol{I}) \times \nabla\right)\right]_{1-\epsilon} \\
& \left.\hat{U}_{212}^{t r C}(\boldsymbol{R})=\frac{\hat{J}_{t r}(Q)}{5 \sqrt{\pi}} u_{1 \frac{3}{2}}(R)\left[\hat{g}_{01}-\hat{g}_{21}\right] u_{1 \frac{3}{2}}(R)\left[Y_{1}(\hat{\boldsymbol{R}}) \times \tau_{1}\left(\boldsymbol{\sigma}_{0}\right)\right]_{2 \epsilon}\left[\tau_{2}(\boldsymbol{I}) \times \nabla\right)\right]_{2-\epsilon}
\end{aligned}
$$




$$
\begin{aligned}
& \hat{U}_{312}^{t r C}(\boldsymbol{R})=\frac{-\hat{J}_{t r}(Q)}{50 \sqrt{\pi}} u_{1 \frac{3}{2}}(R)\left[5 \hat{g}_{01}+\hat{g}_{21}\right] u_{1 \frac{3}{2}}(R)\left[Y_{1}(\hat{\boldsymbol{R}}) \times \tau_{1}\left(\boldsymbol{\sigma}_{0}\right)\right]_{2 \epsilon}\left[\tau_{3}(\boldsymbol{I}) \times \nabla\right]_{2-\epsilon}, \\
& \hat{U}_{332}^{t r C}(\boldsymbol{R})=\frac{53 \sqrt{6} \hat{J}_{t r}(Q)}{175 \sqrt{\pi}} u_{1 \frac{3}{2}}(R)\left[\hat{g}_{21} u_{1 \frac{3}{2}}(R)\right]\left[Y_{3}(\hat{\boldsymbol{R}}) \times \tau_{1}\left(\boldsymbol{\sigma}_{0}\right)\right]_{2 \epsilon}\left[\tau_{3}(\boldsymbol{I}) \times \nabla\right]_{2-\epsilon} \\
& \hat{U}_{333}^{t r C}(\boldsymbol{R})=\frac{-11 \sqrt{3} \hat{J}_{t r}(Q)}{35 \sqrt{2 \pi}} u_{1 \frac{3}{2}}(R)\left[\hat{g}_{21} u_{1 \frac{3}{2}}(R)\right]\left[Y_{3}(\hat{\boldsymbol{R}}) \times \tau_{1}\left(\boldsymbol{\sigma}_{0}\right)\right]_{3 \epsilon}\left[\tau_{3}(\boldsymbol{I}) \times \nabla\right]_{3-\epsilon} \\
& \hat{U}_{334}^{t r C}(\boldsymbol{R})=\frac{3 \sqrt{3} \hat{J}_{t r}(Q)}{35 \sqrt{2 \pi}} u_{1 \frac{3}{2}}(R)\left[\hat{g}_{21} u_{1 \frac{3}{2}}(R)\right]\left[Y_{3}(\hat{\boldsymbol{R}}) \times \tau_{1}\left(\boldsymbol{\sigma}_{0}\right)\right]_{4 \epsilon}\left[\tau_{3}(\boldsymbol{I}) \times \nabla\right]_{4-\epsilon}
\end{aligned}
$$

These folded potentials also have a grad operator acting on the initial distorted wavefunction. The modification of the spin-spin amplitude formalism in Chapter 2 for these spin-spin interactions will be discussed in Section 5.4.2.

\subsection{Modified Scattering Amplitudes}

The folded spin-spin potentials derived from two of the three tensor exchange terms in the NN interaction have differential operators which act on the distorted waves so that the DWBA scattering amplitude for the local spin-spin interactions, given by Eq. 2.20, can no longer be used. The modified scattering amplitude for tensor exchange term $\mathrm{B}$ is given by

$$
\begin{aligned}
f_{k_{I} k}^{t r B}\left(\mu_{f}, M_{f} ; \mu_{i}, M_{i} ; \theta, \phi\right)=\frac{-\mu_{c m}}{2 \pi \hbar^{2}}\left\langle\chi_{\mu_{f}}^{(-)}\left(\boldsymbol{R}, \boldsymbol{k}^{\prime}\right), I M_{f}\right| \hat{F}_{k_{I} k}^{t r B}(R) \\
\times\left[Y_{k}(\hat{\boldsymbol{R}}) \times \tau_{k_{I}}(\boldsymbol{I})\right] \cdot\left[\tau_{1}\left(\boldsymbol{\sigma}_{0}\right) \times \mathscr{Y}_{2}\left(-\imath \nabla_{\boldsymbol{R}}\right)\right]\left|\chi_{\mu_{i}}^{(+)}(\boldsymbol{R}, \boldsymbol{k}), I M_{i}\right\rangle,
\end{aligned}
$$

and for term $\mathrm{C}$

$$
\begin{aligned}
& f_{k_{I} k}^{t r C}\left(\mu_{f}, M_{f} ; \mu_{i}, M_{i} ; \theta, \phi\right)=\frac{-\mu_{c m}}{2 \pi \hbar^{2}}\left\langle\chi_{\mu_{f}}^{(-)}\left(\boldsymbol{R}, \boldsymbol{k}^{\prime}\right), I M_{f}\right| \sum_{E} \hat{F}_{k_{I} k E}^{t r C}(R) \\
& \times\left[Y_{k}(\hat{\boldsymbol{R}}) \times \tau_{1}\left(\boldsymbol{\sigma}_{0}\right)\right]_{E \epsilon}\left[\tau_{k_{I}}(\boldsymbol{I}) \times \nabla\right]_{E-\epsilon}\left|\chi_{\mu_{i}}^{(+)}(\boldsymbol{R}, \boldsymbol{k}), I M_{i}\right\rangle .
\end{aligned}
$$

In order to evaluate these amplitudes the effect of $\mathscr{Y}_{2 \mu}\left(-\imath \nabla_{R}\right)$ and $\nabla_{\mu_{3}}$ on the initial distorted wave $\chi_{\mu_{i}}^{(+)}(\boldsymbol{R}, \boldsymbol{k})$ given in Eq. 2.21 must be determined. Writing out $\chi_{\mu_{i}}^{(+)}(\boldsymbol{R}, \boldsymbol{k})$ in full using the spin-angle function given by Eq. 2.22 leads to

$$
\begin{aligned}
\chi_{\mu_{i}}^{(+)}(\boldsymbol{R}, \boldsymbol{k}) & =4 \pi \sum_{\ell, m_{\ell}, j, m_{j}} i^{\ell} e^{i \sigma_{\ell}} \frac{u_{\ell, j}\left(k_{c m} R\right)}{k_{c m} R}\left(\ell m_{\ell} \frac{1}{2} \mu_{i} \mid j m_{j}\right) Y_{\ell m_{\ell}}^{*}(\hat{\boldsymbol{k}}) \\
& \times \sum_{m_{\ell}^{\prime \prime}, \mu_{i}^{\prime}}\left(\ell m_{\ell}^{\prime \prime} \frac{1}{2} \mu_{i}^{\prime} \mid j m_{j}\right) \mathcal{X}_{\frac{1}{2} \mu_{i}^{\prime}} Y_{\ell m_{\ell}^{\prime \prime}}(\hat{\boldsymbol{R}})
\end{aligned}
$$


The components of the distorted wavefunction that these differential operators act on are the distorted radial wavefunctions $u_{\ell, j}\left(k_{c m} R\right) / k_{c m} R$ and the spherical harmonic $Y_{\ell m_{\ell}^{\prime \prime}}(\hat{\boldsymbol{R}})$. Eq. D.13 derived in Appendix D.2 shows the result obtained when the solid spherical harmonic acts on a radial function and a spherical harmonic. This can be used to show that

$$
\begin{aligned}
\mathscr{Y}_{2}\left(-\imath \nabla_{\boldsymbol{R}}\right) & \frac{u_{\ell, j}\left(k_{c m} R\right)}{k_{c m} R} Y_{\ell m_{\ell}^{\prime \prime}}(\hat{\boldsymbol{R}}) \\
& =\frac{-\hat{2}}{\sqrt{4 \pi}} \sum_{L M}\left(\ell m_{\ell}^{\prime \prime} 2 \mu \mid L M\right)(20 L 0 \mid \ell 0)\left[\hat{\mathscr{O}}_{L \ell} \frac{u_{\ell, j}\left(k_{c m} R\right)}{k_{c m} R}\right] Y_{L M}(\hat{\boldsymbol{R}}) .
\end{aligned}
$$

Eq. D.5 in Appendix D.2 gives a general expression for $\nabla_{\mu_{3}}$ acting on a radial function and a spherical harmonic and can be used to give

$$
\begin{aligned}
\nabla_{\mu_{3}} & \frac{u_{\ell, j}\left(k_{c m} R\right)}{k_{c m} R} Y_{\ell m_{\ell}^{\prime \prime}}(\hat{\boldsymbol{R}}) \\
& =\sum_{L M}\left(\ell m_{\ell}^{\prime \prime} 1 \mu_{3} \mid L M\right)(-)^{\ell-L} \frac{\hat{\ell}}{\hat{L}}(\ell 010 \mid L 0)\left[-\hat{g}_{L \ell} \frac{u_{\ell, j}\left(k_{c m} R\right)}{k_{c m} R}\right] Y_{L M}(\hat{\boldsymbol{R}}) .
\end{aligned}
$$

Eqs. 5.52 and 5.53 will now be used to derive the modified scattering amplitudes for the tensor exchange terms.

\subsubsection{Tensor Term B}

Substituting all the components into Eq. 5.49 yields

$$
\begin{aligned}
f_{k_{I} k}^{t r B}( & \left.\mu_{f}, M_{f} ; \mu_{i}, M_{i} ; \theta, \phi\right) \\
= & \frac{-\mu_{c m}(4 \pi)^{2}}{2 \pi \hbar^{2}} \int d \boldsymbol{R} \sum_{\ell^{\prime}, m_{\ell}^{\prime}, j^{\prime}, m_{j}^{\prime}} \imath^{-\ell^{\prime}} e^{\imath \sigma_{\ell^{\prime}}} \frac{u_{\ell^{\prime}, j^{\prime}}\left(k_{c m} R\right)}{k_{c m} R}\left(\ell^{\prime} m_{\ell}^{\prime} \frac{1}{2} \mu_{f} \mid j^{\prime} m_{j}^{\prime}\right) Y_{\ell^{\prime} m_{\ell}^{\prime}}\left(\hat{\boldsymbol{k}}^{\prime}\right) \\
& \times \sum_{m_{\ell}^{\prime \prime \prime}, \mu_{f}^{\prime}}\left(\ell^{\prime} m_{\ell}^{\prime \prime \prime} \frac{1}{2} \mu_{f}^{\prime} \mid j^{\prime} m_{j}^{\prime}\right) \mathcal{X}_{\frac{1}{2} \mu_{f}^{\prime}} Y_{\ell^{\prime} m_{\ell}^{\prime \prime \prime}}^{*}(\hat{\boldsymbol{R}}) \hat{F}_{k_{I} k}^{t r B}(R) \sum_{q, q_{I}, \mu_{0}, \mu_{1}, \mu}(-)^{\mu_{1}} \\
& \times\left(k q k_{I} q_{I} \mid 1 \mu_{1}\right) Y_{k q}(\hat{\boldsymbol{R}})\left\langle I M_{f}\left|\tau_{k_{I} q_{I}}(\boldsymbol{I})\right| I M_{i}\right\rangle\left(1 \mu_{0} 2 \mu \mid 1-\mu_{1}\right) \tau_{1 \mu_{0}}\left(\boldsymbol{\sigma}_{0}\right) \\
& \times \sum_{\ell, m_{\ell}, j, m_{j}} \imath^{\ell} e^{\imath \sigma_{\ell}}\left(\ell m_{\ell} \frac{1}{2} \mu_{i} \mid j m_{j}\right) Y_{\ell m_{\ell}}^{*}(\hat{\boldsymbol{k}}) \sum_{m_{\ell}^{\prime \prime}, \mu_{i}^{\prime}}\left(\ell m_{\ell}^{\prime \prime} \frac{1}{2} \mu_{i}^{\prime} \mid j m_{j}\right) \mathcal{X}_{\frac{1}{2} \mu_{i}^{\prime}} \\
& \times \frac{-\hat{2}}{\sqrt{4 \pi}} \sum_{L M}\left(\ell m_{\ell}^{\prime \prime} 2 \mu \mid L M\right)(20 L 0 \mid \ell \ell)\left[\hat{\mathscr{O}}_{L \ell} \frac{u_{\ell, j}\left(k_{c m} R\right)}{k_{c m} R}\right] Y_{L M}(\hat{\boldsymbol{R}}) .
\end{aligned}
$$

Using the angular momentum recoupling techniques detailed in Appendices A, C and D (and in [42]), choosing the incident beam direction to be along the z-axis with the yaxis perpendicular to the scattering plane (along $\boldsymbol{k} \times \boldsymbol{k}^{\prime}$ ) and using expressions from [90], 
reduces Eq. 5.54 to

$$
\begin{aligned}
& f_{k_{I} k}^{\operatorname{tr} B}\left(\mu_{f}, M_{f} ; \mu_{i}, M_{i} ; \theta\right) \\
& =\frac{3 \sqrt{30}}{E_{c m} \sqrt{4 \pi}} \hat{k_{I}} \hat{k} \sum_{\ell^{\prime}, \ell, j^{\prime}, j}(-)^{j-\ell^{\prime}+1 / 2-2 \mu_{i}-q_{I}} \hat{\ell} \hat{j} \imath^{\ell-\ell^{\prime}} e^{\imath\left(\sigma_{\ell}+\sigma_{\ell^{\prime}}\right)} Y_{\ell^{\prime}, \mu_{i}-q_{I}-\mu_{f}}\left(\hat{\boldsymbol{k}^{\prime}}\right)\left(\ell 0 \frac{1}{2} \mu_{i} \mid j \mu_{i}\right) \\
& \times\left(\ell^{\prime} \mu_{i}-q_{I}-\mu_{f} \frac{1}{2} \mu_{f} \mid j^{\prime} \mu_{i}-q_{I}\right)\left(I M_{i} k_{I} q_{I} \mid I M_{f}\right)\left(k_{I}-q_{I} j \mu_{i} \mid j^{\prime} \mu_{i}-q_{I}\right) \\
& \times \int_{0}^{\infty} R d R u_{\ell^{\prime}, j^{\prime}}\left(k_{c m} R\right) \hat{F}_{k_{I} k}^{t r B}(R) \sum_{L E}\left[\hat{\mathscr{O}}_{L \ell} \frac{u_{\ell, j}\left(k_{c m} R\right)}{R}\right] \hat{L}^{2}(20 L 0 \mid \ell 0)\left(k 0 L 0 \mid \ell^{\prime} 0\right) \\
& \times \hat{E}^{2} W\left(k_{I} E j^{\prime} \frac{1}{2} ; \ell^{\prime} j\right)\left\{\begin{array}{ccc}
k_{I} & E & \ell^{\prime} \\
L & k & 1
\end{array}\right\}\left\{\begin{array}{ccc}
j & E & \frac{1}{2} \\
1 & \frac{1}{2} & \ell
\end{array}\right\}\left\{\begin{array}{ccc}
1 & E & \ell \\
L & 2 & 1
\end{array}\right\} .
\end{aligned}
$$

\subsubsection{Tensor Term C}

Substituting the distorted wavefunction and folded potential into Eq. 5.50 yields

$$
\begin{aligned}
& f_{k_{I} k}^{t r}\left(\mu_{f}, M_{f} ; \mu_{i}, M_{i} ; \theta, \phi\right) \frac{-\mu_{c m}(4 \pi)^{2}}{2 \pi \hbar^{2}} \int d \boldsymbol{R} \sum_{\ell^{\prime}, m_{\ell}^{\prime}, j^{\prime}, m_{j}^{\prime}} \imath^{-\ell^{\prime}} e^{\imath \sigma_{\ell^{\prime}}} \frac{u_{\ell^{\prime}, j^{\prime}}\left(k_{c m} R\right)}{k_{c m} R}\left(\ell^{\prime} m_{\ell}^{\prime} \frac{1}{2} \mu_{f} \mid j^{\prime} m_{j}^{\prime}\right) Y_{\ell^{\prime} m_{\ell}^{\prime}}\left(\hat{\boldsymbol{k}}^{\prime}\right) \\
& \quad \times \sum_{m_{\ell}^{\prime \prime \prime}, \mu_{f}^{\prime}}\left(\ell^{\prime} m_{\ell}^{\prime \prime \prime} \frac{1}{2} \mu_{f}^{\prime} \mid j^{\prime} m_{j}^{\prime}\right) \mathcal{X}_{\frac{1}{2} \mu_{f}^{\prime}} Y_{\ell^{\prime} m_{\ell}^{\prime \prime \prime}}(\hat{\boldsymbol{R}}) \sum_{E} \hat{F}_{k_{I} k E}^{t r C}(R) \sum_{q q_{I} \mu_{0} \mu_{2}^{\prime}}(-1)^{\epsilon} \\
& \quad \times\left(k q 1 \mu_{0} \mid E \epsilon\right) Y_{k q}(\hat{\boldsymbol{R}}) \tau_{1 \mu_{0}}\left(\boldsymbol{\sigma}_{0}\right)\left(k_{I} q_{I} 1 \mu_{3} \mid E-\epsilon\right)\left\langle I M_{f}\left|\tau_{k_{I} q_{I}}(\boldsymbol{I})\right| I M_{i}\right\rangle \\
& \times \sum_{\ell, m_{\ell}, j, m_{j}} \imath^{\ell} e^{\imath \sigma_{\ell}}\left(\ell m_{\ell} \frac{1}{2} \mu_{i} \mid j m_{j}\right) Y_{\ell m_{\ell}}^{*}(\hat{\boldsymbol{k}}) \sum_{m_{\ell}^{\prime \prime}, \mu_{i}^{\prime}}\left(\ell m_{\ell}^{\prime \prime} \frac{1}{2} \mu_{i}^{\prime} \mid j m_{j}\right) \mathcal{X}_{\frac{1}{2} \mu_{i}^{\prime}} \\
& \quad \times \sum_{L M}\left(\ell m_{\ell}^{\prime \prime} 1 \mu_{3} \mid L M\right)(-)^{\ell-L} \frac{\hat{\ell}}{\hat{L}}(\ell 010 \mid L 0)\left[-\hat{g}_{L \ell} \frac{u_{\ell, j}\left(k_{c m} R\right)}{k_{c m} R}\right] Y_{L M}(\hat{\boldsymbol{R}}) .
\end{aligned}
$$

Using the same recoupling methods and choice of axes as term B, Eq. 5.56 gives

$$
\begin{aligned}
& f_{k_{I} k}^{t r C}\left(\mu_{f}, M_{f} ; \mu_{i}, M_{i} ; \theta\right) \\
& \quad=\frac{-\sqrt{6}}{E_{c m}} \hat{k_{I}} \hat{k} \sum_{\ell^{\prime}, \ell, j^{\prime}, j} \hat{\ell}^{2} \hat{j} \imath^{\ell-\ell^{\prime}}(-)^{\ell+j-j^{\prime}+2 \mu_{i}-k_{I}-q_{I}} e^{\imath\left(\sigma_{\ell}+\sigma_{\ell^{\prime}}\right)} Y_{\ell^{\prime}, \mu_{i}-q_{I}-\mu_{f}}\left(\hat{\boldsymbol{k}}^{\prime}\right)\left(\ell 0 \frac{1}{2} \mu_{i} \mid j \mu_{i}\right) \\
& \quad \times\left(\ell^{\prime} \mu_{i}-q_{I}-\mu_{f} \frac{1}{2} \mu_{f} \mid j^{\prime} \mu_{i}-q_{I}\right)\left(I M_{i} k_{I} q_{I} \mid I M_{f}\right)\left(k_{I}-q_{I} j \mu_{i} \mid j^{\prime} \mu_{i}-q_{I}\right) \\
& \quad \times \int_{0}^{\infty} R d R u_{\ell^{\prime}, j^{\prime}}\left(k_{c m} R\right) \sum_{E L} \hat{F}_{k_{I} k E}^{t r C}(R)\left[\hat{g}_{L \ell} \frac{u_{\ell, j}\left(k_{c m} R\right)}{R}\right](-)^{-L} \hat{L} \hat{E}^{2}(\ell 010 \mid L 0) \\
& \quad \times\left(k 0 L 0 \mid \ell^{\prime} 0\right) \sum_{E^{\prime}} \hat{E}^{{ }^{2}} W\left(j \frac{1}{2} E^{\prime} 1 ; \ell \frac{1}{2}\right) W\left(j \frac{\frac{1}{2}}{2} k_{I} \ell^{\prime} ; E^{\prime} j^{i}\right)\left\{\begin{array}{ccc}
1 & \ell & E^{\prime} \\
E & 1 & k_{I} \\
k & L & \ell^{\prime}
\end{array}\right\} .
\end{aligned}
$$




\subsection{Exchange Folding Checks}

\subsubsection{Checks of Folded Potentials from Central Exchange NN Term}

The folded potential from the central exchange term can be checked in the same way as the direct term, by using the volume integral as discussed in Section 3.9.1. The deltafunction form of the pseudo-potential $\hat{J}_{\sigma \sigma}(Q) \delta\left(\boldsymbol{r}_{p}\right) \boldsymbol{\sigma}_{0} \cdot \boldsymbol{\sigma}_{1} P_{01}^{r}$, used to approximate the exchange term makes this check simpler than the direct term. The folded potential from this exchange term has the form $\hat{U}_{k_{I} k}^{\sigma \sigma}(\boldsymbol{R})=\hat{F}_{k_{I} k}^{\sigma \sigma}(R) \boldsymbol{S}_{k_{I} k}$.

For $k_{I}=1$ and $k=0$ the volume integral of the folded potential is given by the following

$$
\begin{aligned}
& 4 \pi \int_{0}^{\infty} R^{2} d R \hat{F}_{10}^{\sigma \sigma}(R)\left\langle I, M_{f}\left|\boldsymbol{S}_{10}\right| I, M_{i}\right\rangle \\
& =\iiint d \boldsymbol{R} d \boldsymbol{r}_{1} d \boldsymbol{r}_{2} \Psi_{I, M_{f}}^{*}\left(\boldsymbol{r}_{1}, \boldsymbol{r}_{2}\right) \hat{J}_{\sigma \sigma}(Q) \delta\left(\boldsymbol{R}-\boldsymbol{r}_{1}\right) \boldsymbol{\sigma}_{0} \cdot \boldsymbol{\sigma}_{1} P_{01}^{r} \Psi_{I, M_{i}}\left(\boldsymbol{r}_{1}, \boldsymbol{r}_{2}\right), \\
& =\hat{J}_{\sigma \sigma}(Q) \iint d \boldsymbol{R} d \boldsymbol{r}_{2} \Psi_{I, M_{f}}^{*}\left(\boldsymbol{R}, \boldsymbol{r}_{2}\right) \boldsymbol{\sigma}_{0} \cdot \boldsymbol{\sigma}_{1} \Psi_{I, M_{i}}\left(\boldsymbol{R}, \boldsymbol{r}_{2}\right), \\
& =\hat{J}_{\sigma \sigma}(Q) \times A^{\sigma \sigma}\left\langle I, M_{f}\left|\boldsymbol{S}_{10}\right| I, M_{i}\right\rangle, \\
& \Rightarrow \int_{0}^{\infty} R^{2} d R \hat{F}_{10}^{\sigma \sigma}(R)=\hat{J}_{\sigma \sigma}(Q) \times A^{\sigma \sigma}
\end{aligned}
$$

so the volume integral of this folded potential is simply related to the product of the coefficients $\hat{J}_{\sigma \sigma}(Q)$ and $A^{\sigma \sigma}$, where the coefficient $A^{\sigma \sigma}$ is the same as that derived for the direct term given by Eq. 3.63 in Section 3.9.1.

For $k_{I}=1$ or 3 and $k=2$ to give a non-zero result, the folded potential is multiplied by $R^{2} Y_{2 q^{\prime}}^{*}(\hat{\boldsymbol{R}})$ then integrated over all space in the same process as the check for the direct terms. For the exchange terms this method gives

$$
\begin{aligned}
& \int d \boldsymbol{R} \hat{F}_{k_{I} 2}^{\sigma \sigma}(R)\left\langle I, M_{f}\left|\boldsymbol{S}_{k_{I} 2}\right| I, M_{i}\right\rangle R^{2} Y_{2 q^{\prime}}^{*}(\hat{\boldsymbol{R}}) \\
& =\int d \boldsymbol{R} \hat{F}_{k_{I} 2}^{\sigma \sigma}(R) \sum_{\mu_{0} q_{I} q}\left(1 \mu_{0} k_{I} q_{I} \mid 2-q\right) \tau_{1 \mu_{0}}\left(\boldsymbol{\sigma}_{0}\right)\left\langle I, M_{f}\left|\tau_{k_{I} q_{I}}(\boldsymbol{I})\right| I, M_{i}\right\rangle Y_{2 q}(\hat{\boldsymbol{R}})(-)^{q} R^{2} Y_{2 q^{\prime}}^{*}(\hat{\boldsymbol{R}}) \\
& =\iiint d \boldsymbol{R} d \boldsymbol{r}_{1} d \boldsymbol{r}_{2} \Psi_{I, M_{f}}^{*}\left(\boldsymbol{r}_{1}, \boldsymbol{r}_{2}\right) \hat{J}_{\sigma \sigma}(Q) \delta\left(\boldsymbol{R}-\boldsymbol{r}_{1}\right) \boldsymbol{\sigma}_{0} \cdot \boldsymbol{\sigma}_{1} P_{01}^{r} \\
& \quad \times \Psi_{I, M_{i}}\left(\boldsymbol{r}_{1}, \boldsymbol{r}_{2}\right) R^{2} Y_{2 q^{\prime}}^{*}(\hat{\boldsymbol{R}}) .
\end{aligned}
$$


Integrating on both sides leads to

$$
\begin{aligned}
\int_{0}^{\infty} & R^{4} d R \hat{F}_{k_{I} 2}^{\sigma \sigma}(R) \sum_{\mu_{0} q_{I} q}\left(1 \mu_{0} k_{I} q_{I} \mid 2-q\right) \tau_{1 \mu_{0}}\left(\boldsymbol{\sigma}_{0}\right)\left\langle I, M_{f}\left|\tau_{k_{I} q_{I}}(\boldsymbol{I})\right| I, M_{i}\right\rangle(-)^{q} \\
& =\hat{J}_{\sigma \sigma}(Q) \iint d \boldsymbol{R} d \boldsymbol{r}_{2} \Psi_{I, M_{f}}^{*}\left(\boldsymbol{R}, \boldsymbol{r}_{2}\right) \boldsymbol{\sigma}_{0} \cdot \boldsymbol{\sigma}_{1} \Psi_{I, M_{i}}\left(\boldsymbol{R}, \boldsymbol{r}_{2}\right) R^{2} Y_{2 q^{\prime}}^{*}(\hat{\boldsymbol{R}}) \\
& =\hat{J}_{\sigma \sigma}(Q) \times B_{k_{I}}^{\sigma \sigma} \sum_{\mu_{0} q_{I}}\left(1 \mu_{0} k_{I} q_{I} \mid 2-q\right) \tau_{1 \mu_{0}}\left(\boldsymbol{\sigma}_{0}\right)\left\langle I, M_{f}\left|\tau_{k_{I} q_{I}}(\boldsymbol{I})\right| I, M_{i}\right\rangle(-)^{q} \\
\Rightarrow & \int R^{4} d R \hat{F}_{k_{I} 2}^{\sigma \sigma}(R)=\hat{J}_{\sigma \sigma}(Q) \times B_{k_{I}}^{\sigma \sigma}
\end{aligned}
$$

This time the folded potentials are simply related to the product of the coefficients $\hat{J}_{\sigma \sigma}(Q)$ and $B_{k_{I}}^{\sigma \sigma}$. The coefficient $B_{k_{I}}^{\sigma \sigma}$ is derived in Section 3.9.1 for the direct central NN interaction term and is given in Eq. 3.69.

The checks detailed in this section can be applied to all the potentials derived from the folding of the central exchange term in the NN interaction given in Eqs. $5.3-5.5$.

\subsubsection{Checks of Folded Potentials from Tensor Exchange NN Term}

The form of the tensor exchange term in the NN interaction makes checking the resulting folded potential using volume integrals more difficult. A different method has therefore been adopted to check the folded potentials from the tensor exchange terms. An alternative calculation is performed to not only check the folded potentials from all three tensor exchange terms but also to investigate their time reversal invariance.

Generally using standard vector identities it can be shown that

$$
A \nabla^{2} B=A \operatorname{div}(\nabla B)=\operatorname{div}(A \nabla B)-\nabla A \cdot \nabla B
$$

In our case, the rank 2 solid spherical harmonic in the tensor exchange pseudo-potential acts on the function $B$, which is composed of the final valence-nucleon wavefunction and the initial distorted wavefunction. Integrating Eq. 5.61 over all space, (as is done when evaluating the spin-spin amplitudes), gives

$$
\int d \boldsymbol{R} A \nabla^{2} B=\int d \boldsymbol{R} \operatorname{div}(A \nabla B)-\int d \boldsymbol{R} \nabla A \cdot \nabla B
$$

The first term on the right hand side of Eq. 5.62 can be evaluated using divergence theorem. This term is then equal to the surface integral at infinity of the function $A \nabla B$ dotted with the unit vector perpendicular with the surface. For bound state wavefunctions, which 
tend to zero as $r \rightarrow 0$ and $r \rightarrow \infty$, this term is zero and we get

$$
\int d \boldsymbol{R} A \nabla^{2} B=-\int d \boldsymbol{R} \nabla A \cdot \nabla B
$$

This can be applied to the folding of the tensor exchange term. Taking the coordinate dependent parts of the folding equation for the tensor exchange term, Eq. 5.12, including the distorted waves, leads to Eq. 5.16, which can be written as

$$
\frac{-\sqrt{15}}{\sqrt{8 \pi}} \sum_{\mu_{2} \mu_{3}}\left(1 \mu_{2} 1 \mu_{3} \mid 2 \mu\right) \chi_{\mu_{f}}^{*(-)}\left(\boldsymbol{R}, \boldsymbol{k}^{\prime}\right) Y_{\ell_{1} \lambda_{1}}(\hat{\boldsymbol{R}}) u_{\ell_{1} j_{1}}(R) \vec{\nabla}_{\mu_{2}} \vec{\nabla}_{\mu_{3}} Y_{\ell_{1}^{\prime} \lambda_{1}^{\prime}}^{*}(\hat{\boldsymbol{R}}) u_{\ell_{1}^{\prime} j_{1}^{\prime}}(R) \chi_{\mu_{i}}^{(+)}(\boldsymbol{R}, \boldsymbol{k})
$$

where Eq. 5.11 has been used for $\mathscr{Y}_{2 \mu}\left(-\imath \nabla_{\boldsymbol{r}_{N}}\right)$ and the arrows on the grad operators indicate the direction in which they act. Integrating Eq. 5.64 over $R$ and using the result from Eq. 5.63, gives

$$
\begin{aligned}
\frac{\sqrt{15}}{\sqrt{8 \pi}} \sum_{\mu_{2} \mu_{3}}\left(1 \mu_{2} 1 \mu_{3} \mid 2 \mu\right) \int & d \boldsymbol{R} \chi_{\mu_{f}}^{*(-)}\left(\boldsymbol{R}, \boldsymbol{k}^{\prime}\right) Y_{\ell_{1} \lambda_{1}}(\hat{\boldsymbol{R}}) u_{\ell_{1} j_{1}}\left(R_{i}\right) \overleftarrow{\nabla} \mu_{\mu_{2}} \\
\times & \vec{\nabla}_{\mu_{3}} Y_{\ell_{1}^{\prime} \lambda_{1}^{\prime}}^{*}(\hat{\boldsymbol{R}}) u_{\ell_{1}^{\prime} j_{1}^{\prime}}(R) \chi_{\mu_{i}}^{(+)}(\boldsymbol{R}, \boldsymbol{k}) .
\end{aligned}
$$

This leads to four separate terms; i) where the grads act on the components of both valence nucleon wavefunctions, ii) where the grads both act on the distorted waves, iii) where the left grad acts on the final distorted wavefunction and the right grad acts on the components of the initial valence nucleon wavefunction and iv) where the left grad acts on the components of the final valence nucleon wavefunction and the right grad acts on the initial distorted wavefunction:

$$
\begin{aligned}
& \frac{\sqrt{15}}{\sqrt{8 \pi}} \sum_{\mu_{2} \mu_{3}}\left(1 \mu_{2} 1 \mu_{3} \mid 2 \mu\right) \int d \boldsymbol{R} \\
& \quad \times\left\{\chi_{\mu_{f}}^{*(-)}\left(\boldsymbol{R}, \boldsymbol{k}^{\prime}\right)\left[\nabla_{\mu_{2}} Y_{\ell_{1} \lambda_{1}}(\hat{\boldsymbol{R}}) u_{\ell_{1} j_{1}}(R)\right]\left[\nabla_{\mu_{3}} Y_{\ell_{1}^{\prime} \lambda_{1}^{\prime}}^{*}(\hat{\boldsymbol{R}}) u_{\ell_{1}^{\prime} j_{1}^{\prime}}(R)\right] \chi_{\mu_{i}}^{(+)}(\boldsymbol{R}, \boldsymbol{k})\right. \\
& \\
&+\left[\nabla_{\mu_{2}} \chi_{\mu_{f}}^{*(-)}\left(\boldsymbol{R}, \boldsymbol{k}^{\prime}\right)\right] Y_{\ell_{1} \lambda_{1}}(\hat{\boldsymbol{R}}) u_{\ell_{1} j_{1}}(R) Y_{\ell_{1}^{\prime} \lambda_{1}^{\prime}}^{*}(\hat{\boldsymbol{R}}) u_{\ell_{1}^{\prime} j_{1}^{\prime}}(R)\left[\nabla_{\mu_{3}} \chi_{\mu_{i}}^{(+)}(\boldsymbol{R}, \boldsymbol{k})\right] \\
&+\left[\nabla_{\mu_{2}} \chi_{\mu_{f}}^{*(-)}\left(\boldsymbol{R}, k^{\prime}\right)\right] Y_{\ell_{1} \lambda_{1}}(\hat{\boldsymbol{R}}) u_{\ell_{1} j_{1}}(R)\left[\nabla_{\mu_{3}} Y_{\ell_{1}^{\prime} \lambda_{1}^{\prime}}^{*}(\hat{\boldsymbol{R}}) u_{\ell_{1} j_{1}^{\prime}}(R)\right] \chi_{\mu_{i}}^{(+)}\left(\boldsymbol{R}, k^{\prime}\right) \\
&\left.+\chi_{\mu_{f}}^{*(-)}\left(\boldsymbol{R}, k^{\prime}\right)\left[\nabla_{\mu_{2}} Y_{\ell_{1} \lambda_{1}}(\hat{\boldsymbol{R}}) u_{\ell_{1} j_{1}}(R)\right] Y_{\ell_{1}^{\prime} \lambda_{1}^{\prime}}^{*}(\hat{\boldsymbol{R}}) u_{\ell_{1}^{\prime} j_{1}^{\prime}}(R)\left[\nabla_{\mu_{3}} \chi_{\mu_{i}}^{(+)}(\boldsymbol{R}, \boldsymbol{k})\right]\right\} .
\end{aligned}
$$

Not only do these four terms allow a check of the spin-spin interactions from the folding of the tensor exchange term in the NN interaction but their symmetry allows the timereversal invariance of their spin-spin amplitudes to be tested (see Section 5.5.8).

Substituting Eq. 5.66 into the folding equation for tensor exchange terms, 5.12, gives 
an alternative to Eq. 5.18

$$
\begin{aligned}
& \int d \boldsymbol{R} \chi_{\mu_{f}}^{*(-)}\left(\boldsymbol{R}, \boldsymbol{k}^{\prime}\right)\left\langle\Psi_{I, M_{f}}\left(\boldsymbol{r}_{1}, \boldsymbol{r}_{2}\right)\right| \hat{J}_{t r}(Q)\left[\hat{\boldsymbol{S}}_{01}\left(\boldsymbol{\sigma}_{0}, \boldsymbol{\sigma}_{1},-\imath \nabla_{\boldsymbol{r}_{p}}\right) \delta\left(\boldsymbol{r}_{p}\right)\right] \\
& \times P_{01}^{r}\left|\Psi_{I, M_{i}}\left(\boldsymbol{r}_{1}, \boldsymbol{r}_{2}\right)\right\rangle \chi_{\mu_{i}}^{(+)}(\boldsymbol{R}, \boldsymbol{k}) \\
& =(-)^{3 j_{1}-j_{1}^{\prime}-\ell_{1}^{\prime}} \hat{J}_{t r}(Q) 3 \sqrt{6} \hat{I} \hat{j}_{1} \hat{j}_{1}^{\prime} \sum_{\substack{\mu \mu_{0} \mu_{1} \\
\lambda_{1}^{\prime} \lambda_{1} \mu_{2} \mu_{3}}}\left(1 \mu_{0} 1 \mu_{1} \mid 2-\mu\right)\left(1 \mu_{2} 1 \mu_{3} \mid 2 \mu\right) \tau_{1 \mu_{0}}\left(\boldsymbol{\sigma}_{0}\right) \int d \boldsymbol{R} \\
& \times\left\{\chi_{\mu_{f}}^{*(-)}\left(\boldsymbol{R}, k^{\prime}\right)\left[\nabla_{\mu_{2}} Y_{\ell_{1} \lambda_{1}}(\hat{\boldsymbol{R}}) u_{\ell_{1} j_{1}}(R)\right]\left[\nabla_{\mu_{3}} Y_{\ell_{1}^{\prime} \lambda_{1}^{\prime}}^{*}(\hat{\boldsymbol{R}}) u_{\ell_{1}^{\prime} j_{1}^{\prime}}(R)\right] \chi_{\mu_{i}}^{(+)}(\boldsymbol{R}, \boldsymbol{k}) \quad i\right. \\
& +\left[\nabla_{\mu_{2}} \chi_{\mu_{f}}^{*(-)}\left(\boldsymbol{R}, \boldsymbol{k}^{\prime}\right)\right] Y_{\ell_{1} \lambda_{1}}(\hat{\boldsymbol{R}}) u_{\ell_{1} j_{1}}(R) Y_{\ell_{1}^{\prime} \lambda_{\mathbf{1}}^{\prime}}^{*}(\hat{\boldsymbol{R}}) u_{\ell_{1}^{\prime} j_{1}^{\prime}}(R)\left[\nabla_{\mu_{3}} \chi_{\mu_{i}}^{(+)}(\boldsymbol{R}, \boldsymbol{k})\right] \quad i i \\
& +\left[\nabla_{\mu_{2}} \chi_{\mu_{f}}^{*(-)}\left(R, k^{\prime}\right)\right] Y_{\ell_{1} \lambda_{1}}(\hat{R}) u_{\ell_{1} j_{1}}(R)\left[\nabla_{\mu_{3}} Y_{\ell_{1}^{\prime} \lambda_{1}^{\prime}}(\hat{R}) u_{\ell_{1}^{\prime} j_{1}^{\prime}}(R)\right] \chi_{\mu_{i}}^{(+)}(R, k) \quad \text { iii } \\
& \left.+\chi_{\mu_{f}}^{*(-)}\left(\boldsymbol{R}, \boldsymbol{k}^{\prime}\right)\left[\nabla_{\mu_{2}} Y_{\ell_{1} \lambda_{1}}(\hat{\boldsymbol{R}}) u_{\ell_{1} j_{1}}(R)\right] Y_{\ell_{1}^{\prime} \lambda_{1}^{\prime}}^{*}(\hat{\boldsymbol{R}}) u_{\ell_{1}^{\prime} j_{1}^{\prime}}(R)\left[\nabla_{\mu_{3}} \chi_{\mu_{i}}^{(+)}(\boldsymbol{R}, k)\right]\right\} \quad i v \\
& \times(-)^{\mu+\mu_{1}-\lambda_{1}} \sum_{k_{I} q_{I}}{\hat{k_{I}}}^{2} W\left(k_{I} j_{1} I j_{2} ; j_{1}^{\prime} I\right)\left(I M_{i} k_{I} q_{I} \mid I M_{f}\right) \sum_{k_{2} q_{2}}(-)^{-k_{2}} \\
& \times\left(\ell_{1}-\lambda_{1} \ell_{1}^{\prime} \lambda_{1}^{\prime} \mid k_{2} q_{2}\right)\left(1-\mu_{1} k_{I} q_{I} \mid k_{2} q_{2}\right)\left\{\begin{array}{ccc}
j_{1} & j_{1}^{\prime} & k_{I} \\
\frac{1}{2} & \frac{1}{2} & 1 \\
\ell_{1} & \ell_{1}^{\prime} & k_{2}
\end{array}\right\} .
\end{aligned}
$$

These four terms will be evaluated separately. The integral over $\boldsymbol{R}$ will be neglected for the discussion of these folded potentials and will be included again for the calculation the spin-spin amplitudes.

\subsubsection{Spin-Spin Potentials for Tensor Exchange Term i}

Tensor Term i will denote the term where the grads act on the components of the valence nucleon wavefunctions and not on the distorted waves (which will therefore be left out in this section). This term in Eq. 5.67 gives

\section{Term i}

$$
\begin{aligned}
& =(-)^{3 j_{1}-j_{1}^{\prime}-\ell_{1}^{\prime}} \hat{J}_{t r}(Q) 3 \sqrt{6} \hat{I} \hat{j}_{1} \hat{j}_{1}^{\prime} \sum_{\substack{\mu \mu_{0} \mu_{1} \\
\lambda_{1}^{\prime} \lambda_{1} \mu_{2} \mu_{3}}}\left(1 \mu_{0} 1 \mu_{1} \mid 2-\mu\right)\left(1 \mu_{2} 1 \mu_{3} \mid 2 \mu\right) \tau_{1 \mu_{0}}\left(\sigma_{0}\right) \\
& \times\left[\nabla_{\mu_{2}} Y_{\ell_{1} \lambda_{1}}(\hat{\boldsymbol{R}}) u_{\ell_{1} j_{1}}(R)\right]\left[\nabla_{\mu_{3}} Y_{\ell_{1}^{\prime} \lambda_{1}^{\prime}}^{*}(\hat{\boldsymbol{R}}) u_{\ell_{1}^{\prime} j_{1}^{\prime}}(R)\right](-)^{\mu+\mu_{1}-\lambda_{1}} \sum_{k_{I} q_{I}} \hat{k}_{I}^{2} W\left(k_{I} j_{1} I j_{2} ; j_{1}^{\prime} I\right) \\
& \times\left(I M_{i} k_{I} q_{I} \mid I M_{f}\right) \sum_{k_{2} q_{2}}(-)^{-k_{2}}\left(\ell_{1}-\lambda_{1} \ell_{1}^{\prime} \lambda_{1}^{\prime} \mid k_{2} q_{2}\right)\left(1-\mu_{1} k_{I} q_{I} \mid k_{2} q_{2}\right)\left\{\begin{array}{ccc}
j_{1} & j_{1}^{\prime} & k_{I} \\
\frac{1}{2} & \frac{1}{2} & 1 \\
\ell_{1} & \ell_{1}^{\prime} & k_{2}
\end{array}\right\} .
\end{aligned}
$$


Using the angular momentum coupling techniques analogous to the procedure detailed in Appendix D.3, Eq. 5.68 can be reduced to

\section{Term i}

$$
\begin{aligned}
& =(-)^{3 j_{1}-j_{1}^{\prime}+\ell_{1}} \hat{J}_{t r}(Q) 30 \sqrt{\frac{3}{8 \pi}} \hat{I} \hat{j}_{1} \hat{j}_{1}^{\prime} \hat{\ell}_{1} \hat{\ell}_{1}^{\prime} \sum_{k_{I} k_{2} L L^{\prime} k}(-)^{-L-L^{\prime}} \frac{\hat{L} \hat{L}^{\prime} \hat{k}_{2}^{2}}{\hat{k}} \\
& \times(-)^{-k_{2}-k_{I}}\left(\ell_{1} 010 \mid L 0\right)\left[\hat{g}_{L \ell_{1}} u_{\ell_{1} j_{1}}(R)\right]\left(\ell_{1}^{\prime} 010 \mid L^{\prime} 0\right)\left[\hat{g}_{L^{\prime} \ell_{1}^{\prime}} u_{\ell_{1}^{\prime} j_{1}^{\prime}}(R)\right]\left(L 0 L^{\prime} 0 \mid k 0\right) \\
& \times W\left(11 k k_{2} ; 2 i\right) \hat{k}_{I} W\left(k_{I} j_{1} I j_{2} ; j_{1}^{\prime} I\right)\left\{\begin{array}{ccc}
j_{1} & j_{1}^{\prime} & k_{I} \\
\frac{1}{2} & \frac{1}{2} & 1 \\
\ell_{1} & \ell_{1}^{\prime} & k_{2}
\end{array}\right\}\left\{\begin{array}{ccc}
1 & 1 & 2 \\
\ell_{1} & \ell_{1} & k_{2} \\
L & L^{\prime} & k
\end{array}\right\}\left\langle I, M_{f}\left|S_{k_{I} k}\right| I, M_{i}\right\rangle .
\end{aligned}
$$

This time, instead of the differential operator $\hat{\mathscr{O}}_{L \ell}$ acting on the radial function of the final valence nucleon wavefunction (as in tensor exchange Term A), a $\hat{g_{L \ell}}$ operator acts on both radial functions. This spin-spin interaction has the form $U_{k_{I} k}(\boldsymbol{R})=F_{k_{I} k}(R) \boldsymbol{S}_{k_{I} k}$ so the spin-spin amplitude for this potential can be calculated using the formalism detailed in Chapter 2.

Using the quantum numbers from the simple ${ }^{10} \mathrm{~B}$ model in Eq. 5.69 and summing over $L, L^{\prime}$ and $k_{2}$ yields four different types of spin-spin interaction

$$
\begin{aligned}
\hat{U}_{10}^{t r i}(R) & =\frac{\hat{J}_{t r}(Q)}{15 \sqrt{3 \pi}}\left\{5\left[\hat{g}_{01} u_{1 \frac{3}{2}}\left(r_{1}\right)\right]\left[\hat{g}_{01} u_{1 \frac{3}{2}}(R)\right]+\left[\hat{g}_{21} u_{1 \frac{3}{2}}\left(r_{1}\right)\right]\left[\hat{g}_{21} u_{1 \frac{3}{2}}(R)\right]\right\} \boldsymbol{S}_{10}, \\
\hat{U}_{12}^{t r i}(\boldsymbol{R}) & =\frac{\hat{J}_{t r}(Q)}{30 \sqrt{30 \pi}}\left\{13\left[\hat{g}_{01} u_{1 \frac{3}{2}}\left(r_{1}\right)\right]\left[\hat{g}_{21} u_{1 \frac{3}{2}}\left(r_{1}\right)\right]+13\left[\hat{g}_{21} u_{1 \frac{3}{2}}\left(r_{1}\right)\right]\left[\hat{g}_{01} u_{1 \frac{3}{2}}(R)\right]\right. \\
& \left.+22\left[\hat{g}_{21} u_{1 \frac{3}{2}}\left(r_{1}\right)\right]\left[\hat{g}_{21} u_{1 \frac{3}{2}}(R)\right]\right\} \boldsymbol{S}_{12}, \\
\hat{U}_{32}^{t r i}(\boldsymbol{R}) & =\frac{\hat{J}_{t r}(Q)}{70 \sqrt{10 \pi}}\left\{7\left[\hat{g}_{01} u_{1 \frac{3}{2}}\left(r_{1}\right)\right]\left[\hat{g}_{21} u_{1 \frac{3}{2}}\left(r_{1}\right)\right]+7\left[\hat{g}_{21} u_{1 \frac{3}{2}}\left(r_{1}\right)\right]\left[\hat{g}_{01} u_{1 \frac{3}{2}}(R)\right]\right. \\
& \left.-2\left[\hat{g}_{21} u_{1 \frac{3}{2}}\left(r_{1}\right)\right]\left[\hat{g}_{21} u_{1 \frac{3}{2}}(R)\right]\right\} \boldsymbol{S}_{32}, \\
\hat{U}_{34}^{t r i}(\boldsymbol{R}) & =\frac{-3 \sqrt{6} \hat{J}_{t r}(Q)}{35 \sqrt{\pi}}\left[\hat{g}_{21} u_{1 \frac{3}{2}}\left(r_{1}\right)\right]\left[\hat{g}_{21} u_{1 \frac{3}{2}}(R)\right] \boldsymbol{S}_{34} .
\end{aligned}
$$

This tensor exchange term is analogous to Term A in that the distorted waves are unaffected by the grad operators. However, tensor exchange Term i only results in four spin-spin potentials. The spin-spin potential, $\hat{U}_{22}(\boldsymbol{R})$ (that has even rank in $\boldsymbol{I}$ ), derived from the folding of Term A, cancels to zero in this case because $\ell^{\prime}=\ell$ and the radial wavefunction is real. 


\subsubsection{Spin-Spin Potentials for Tensor Exchange Term ii}

Tensor Term ii will describe the term in Eq. 5.67 where the grads act on the distorted waves and not on the valence nucleon wavefunction. This is analogous to tensor exchange Term B from the first method. Taking this term only in Eq. 5.67 gives

\section{Term ii}

$$
\begin{aligned}
& =(-)^{3 j_{1}-j_{1}^{\prime}-\ell_{1}^{\prime}} \hat{J}_{t r}(Q) 3 \sqrt{6} \hat{I} \hat{j}_{1} \hat{j}_{1}^{\prime} \sum_{\substack{\mu \mu_{0} \mu_{1} \\
\lambda_{1}^{\prime} \lambda_{1} \mu_{2} \mu_{3}}}\left(1 \mu_{0} 1 \mu_{1} \mid 2-\mu\right)\left(1 \mu_{2} 1 \mu_{3} \mid 2 \mu\right) \tau_{1 \mu_{0}}\left(\boldsymbol{\sigma}_{0}\right) \\
& {\left[\nabla_{\mu_{2}} \chi_{\mu_{f}}^{*(-)}\left(\boldsymbol{R}, \boldsymbol{k}^{\prime}\right)\right] Y_{\ell_{1} \lambda_{1}}(\hat{\boldsymbol{R}}) u_{\ell_{1} j_{1}}(R) Y_{\ell_{1}^{\prime} \lambda_{1}^{\prime}}^{*}(\hat{\boldsymbol{R}}) u_{\ell_{1}^{\prime} j_{1}^{\prime}}(R)\left[\nabla_{\mu_{3}} \chi_{\mu_{i}}^{(+)}(\boldsymbol{R}, \boldsymbol{k})\right]} \\
& \times(-)^{\mu+\mu_{1}-\lambda_{1}} \sum_{k_{I} q_{I}} \hat{k}_{I}^{2} W\left(k_{I} j_{1} I j_{2} ; j_{1}^{\prime} I\right)\left(I M_{i} k_{I} q_{I} \mid I M_{f}\right) \sum_{k_{2} q_{2}}(--)^{-k_{2}} \\
& \times\left(\ell_{1}-\lambda_{1} \ell_{1}^{\prime} \lambda_{1}^{\prime} \mid k_{2} q_{2}\right)\left(1-\mu_{1} k_{I} q_{I} \mid k_{2} q_{2}\right)\left\{\begin{array}{ccc}
j_{1} & j_{1}^{\prime} & k_{I} \\
\frac{1}{2} & \frac{1}{2} & 1 \\
\ell_{1} & \ell_{1}^{\prime} & k_{2}
\end{array}\right\} .
\end{aligned}
$$

The angular momentum coupling techniques detailed in Appendix D.5 for tensor exchange Term B can be used to reduce this to

\section{Term ii}

$$
\begin{aligned}
& =(-)^{1+3 j_{1}-j_{1}^{\prime}-\ell_{1}^{\prime}} \hat{J}_{t r}(Q) 2 \sqrt{\frac{15}{8 \pi}} \hat{I} \hat{j}_{1} \hat{j}_{1}^{\prime} \hat{\ell}_{1} \hat{\ell}_{1}^{\prime} u_{\ell_{1} j_{1}}(R) u_{\ell_{1}^{\prime} j_{1}^{\prime}}(R) \\
& \times \sum_{k_{I} k}(-)^{k_{I}+k}\left(\ell_{1} 0 \ell_{1}^{\prime} 0 \mid k 0\right) \hat{k_{I}} W\left(k_{I} j_{1} I j_{2} ; j_{1}^{\prime} I\right)\left\{\begin{array}{ccc}
j_{1} & j_{1}^{\prime} & k_{I} \\
\frac{1}{2} & \frac{1}{2} & 1 \\
\ell_{1} & \ell_{1}^{\prime} & k_{2}
\end{array}\right\} \\
& \times \sum_{\substack{q q_{I} \mu \mu_{0} \\
\mu_{1} \mu_{2} \mu_{3}}}\left(1 \mu_{2} 1 \mu_{3} \mid 2 \mu\right)\left[\nabla_{\mu_{2}} \chi_{\mu_{f}}^{*(-)}\left(\boldsymbol{R}, \boldsymbol{k}^{\prime}\right)\right]\left(k q k_{I} q_{I} \mid 1 \mu_{1}\right) Y_{k q}(\hat{\boldsymbol{R}})\left\langle I, M_{f}\left|\tau_{k_{I} q_{I}}(\boldsymbol{I})\right| I, M_{i}\right\rangle \\
& \times(-1)^{\mu_{1}}\left(1 \mu_{0} 2 \mu \mid 1-\mu_{1}\right) \tau_{1 \mu_{0}}\left(\boldsymbol{\sigma}_{0}\right)\left[\nabla_{\mu_{3}} \chi_{\mu_{i}}^{(+)}(\boldsymbol{R}, \boldsymbol{k})\right] .
\end{aligned}
$$

As with tensor exchange Term B, Term ii has a different form to the potentials $U_{k_{I} k}(\boldsymbol{R})=$ $F_{k_{I} k}(R) \boldsymbol{S}_{k_{I} k}$ derived previously. The folded potentials from Term ii will therefore be denoted by $\hat{U}_{k_{I} k}^{t r i i}=\hat{F}_{k_{I} k}^{t r i i}(R) \boldsymbol{Y}_{k_{I} k}^{t r i i}$, where $\hat{F}_{k_{I} k}^{t r i i}(R)$ is the form factor given by

$$
\begin{aligned}
\hat{F}_{k_{I} k}^{t r i i}(R) & =(-)^{1+3 j_{1}-j_{1}^{\prime}-\ell_{1}^{\prime}} \hat{J}_{t r}(Q) 2 \sqrt{\frac{15}{8 \pi}} \hat{I} \hat{j}_{1} \hat{j}_{1}^{\prime} \hat{\ell}_{1} \hat{\ell}_{1}^{\prime} u_{\ell_{1} j_{1}}(R) u_{\ell_{1}^{\prime} j_{1}^{\prime}}(R) \\
& \times(-)^{k_{I}+k}\left(\ell_{1} 0 \ell_{1}^{\prime} 0 \mid k 0\right) \hat{k_{I}} W\left(k_{I} j_{1} I j_{2} ; j_{1}^{\prime} I\right)\left\{\begin{array}{ccc}
j_{1} & j_{1}^{\prime} & k_{I} \\
\frac{1}{2} & \frac{1}{2} & 1 \\
\ell_{1} & \ell_{1}^{\prime} & k_{2}
\end{array}\right\}
\end{aligned}
$$


and $\boldsymbol{Y}_{k_{I} k}^{\text {trii }}$ is the operator

$$
\begin{aligned}
\chi_{\mu_{f}}^{*(-)}\left(\boldsymbol{R}, \boldsymbol{k}^{\prime}\right) \boldsymbol{Y}_{k_{I} k}^{t r i i} \chi_{\mu_{i}}^{(+)}(\boldsymbol{R}, \boldsymbol{k}) & =\chi_{\mu_{f}}^{*(-)}\left(\boldsymbol{R}, \boldsymbol{k}^{\prime}\right) \sum_{\substack{q q_{I} \mu \mu_{0} \\
\mu_{1} \mu_{2} \mu_{3}}}\left(1 \mu_{2} 1 \mu_{3} \mid 2 \mu\right) \overleftarrow{\nabla_{\mu_{2}}}\left(k q k_{I} q_{I} \mid 1 \mu_{1}\right) Y_{k q}(\hat{\boldsymbol{R}}) \\
& \times \tau_{k_{I} q_{I}}(\boldsymbol{I})(-1)^{\mu_{1}}\left(1 \mu_{0} 2 \mu \mid 1-\mu_{1}\right) \tau_{1 \mu_{0}}\left(\boldsymbol{\sigma}_{0}\right) \vec{\nabla}_{\mu_{3}} \chi_{\mu_{i}}^{(+)}(\boldsymbol{R}, \boldsymbol{k})
\end{aligned}
$$

For our model of ${ }^{10} \mathrm{~B}$ the spin-spin interactions from Eq. 5.75 are

$$
\begin{aligned}
& \hat{U}_{10}^{t r i i}(R)=-\sqrt{\frac{5}{3 \pi}} \hat{J}_{t r}(Q) u_{1 \frac{3}{2}}(R)^{2} \boldsymbol{Y}_{10}^{t r i i}, \\
& \hat{U}_{12}^{t r i i}(\boldsymbol{R})=-\sqrt{\frac{2}{15 \pi}} \hat{J}_{t r}(Q) u_{1 \frac{3}{2}}(R)^{2} \boldsymbol{Y}_{12}^{t r i i}, \\
& \hat{U}_{32}^{t r i i}(\boldsymbol{R})=\frac{3}{\sqrt{10 \pi}} \hat{J}_{t r}(Q) u_{1 \frac{3}{2}}(R)^{2} \boldsymbol{Y}_{32}^{\text {trii }}
\end{aligned}
$$

Tensor Term ii, Term iii and Term iv all have grad operators that act on the distorted wavefunctions. This means their spin-spin amplitudes cannot be calculated using the formalism in Chapter 2. The details of their modified scattering amplitudes will be given in Section 5.5.7.

\subsubsection{Spin-Spin Potentials for Tensor Exchange Term iii}

Tensor exchange Term in will describe the term in Eq. 5.67 where one grad acts on the final distorted wave and the other on the final state valence nucleon wavefunction. Taking this term only in Eq. 5.67 gives

\section{Term iii}

$$
\begin{aligned}
& =(-)^{3 j_{1}-j_{1}^{\prime}-\ell_{1}^{\prime}} \hat{J}_{t r}(Q) 3 \sqrt{6} \hat{I} \hat{j}_{1} \hat{j}_{1}^{\prime} \sum_{\substack{\mu \mu_{0} \mu_{1} \\
\lambda_{1}^{\prime} \lambda_{1} \mu_{2} \mu_{3}}}\left(1 \mu_{0} 1 \mu_{1} \mid 2-\mu\right)\left(1 \mu_{2} 1 \mu_{3} \mid 2 \mu\right) \tau_{1 \mu_{0}}\left(\boldsymbol{\sigma}_{0}\right) \\
& \times\left[\nabla_{\mu_{2}} \chi_{\mu_{f}}^{*(-)}\left(R, k^{\prime}\right)\right] Y_{\ell_{1} \lambda_{1}}(\hat{\boldsymbol{R}}) u_{\ell_{1} j_{1}}(R)\left[\nabla_{\mu_{3}} Y_{\ell_{1}^{\prime} \lambda_{1}^{\prime}}(\hat{\boldsymbol{R}}) u_{\ell_{1}^{\prime} j_{1}^{\prime}}(R)\right] \chi_{\mu_{i}}^{(+)}(\boldsymbol{R}, k) \\
& \times(-)^{\mu+\mu_{1}-\lambda_{1}} \sum_{k_{I} q_{I}} \hat{k}_{I}^{2} W\left(k_{I} j_{1} I j_{2} ; j_{1}^{\prime} I\right)\left(I M_{i} k_{I} q_{I} \mid I M_{f}\right) \sum_{k_{2} q_{2}}(-)^{-k_{2}} \\
& \times\left(\ell_{1}-\lambda_{1} \ell_{1}^{\prime} \lambda_{1}^{\prime} \mid k_{2} q_{2}\right)\left(1-\mu_{1} k_{I} q_{I} \mid k_{2} q_{2}\right)\left\{\begin{array}{ccc}
j_{1} & j_{1}^{\prime} & k_{I} \\
\frac{1}{2} & \frac{1}{2} & 1 \\
\ell_{1} & \ell_{1}^{\prime} & k_{2}
\end{array}\right\} .
\end{aligned}
$$

Using the angular momentum coupling techniques analogous to those detailed in Appendix D. 5 for Term C this can be reduced to 


\section{Term iii}

$$
\begin{aligned}
& =(-)^{3 j_{1}-j_{1}^{\prime}} \hat{J}_{t r}(Q) 15 \sqrt{\frac{3}{2 \pi}} \hat{I} \hat{j}_{1} \hat{j}_{1}^{\prime} \hat{\ell}_{1} \hat{\ell}_{1}^{\prime} u_{\ell_{1} j_{1}}(R) \sum_{k_{I} k_{2} L k E}\left[\hat{g}_{L \ell_{1}^{\prime}} u_{\ell_{1}^{\prime} j_{1}^{\prime}}(R)\right] \\
& \times(-)^{k-L} \hat{L} \hat{k}_{2}^{2}\left(\ell_{1}^{\prime} 010 \mid L 0\right)\left(\ell_{1} 0 L 0 \mid k 0\right) W\left(1 \ell_{1}^{\prime} k \ell_{1} ; L k_{2}\right) \hat{k_{I}} W\left(k_{I} j_{1} I j_{2} ; j_{1}^{\prime} I\right) \\
& \times\left\{\begin{array}{ccc}
j_{1} & j_{1}^{\prime} & k_{I} \\
\frac{1}{2} & \frac{1}{2} & 1 \\
\ell_{1} & \ell_{1}^{\prime} & k_{2}
\end{array}\right\}\left\{\begin{array}{ccc}
1 & k_{I} & E \\
1 & k_{2} & k \\
2 & 1 & 1
\end{array}\right\} \sum_{q q_{I} \mu_{0} \mu_{2} \epsilon}\left[\nabla_{\mu_{2}} \chi_{\mu_{f}}^{*(-)}\left(R, k^{\prime}\right)\right]\left(1 \mu_{2} k_{I} q_{I} \mid E \epsilon\right) \\
& \times\left\langle I, M_{f}\left|\tau_{k_{I}} q_{I}(\boldsymbol{I})\right| I, M_{i}\right\rangle(-1)^{\epsilon}\left(1 \mu_{0} k q \mid E-\epsilon\right) \tau_{1 \mu_{0}}\left(\boldsymbol{\sigma}_{0}\right) Y_{k q}(\hat{\boldsymbol{R}}) \chi_{\mu_{i}}^{(+)}(\boldsymbol{R}, \boldsymbol{k}) .
\end{aligned}
$$

The folded potentials from Term iii will be denoted by $\hat{U}_{k_{I} k E}^{t r i i i}=\hat{F}_{k_{I} k E}^{\text {triii }}(R) Z_{k_{I} k E}^{\text {triv }}$. The extra subscript $E$ represents the coupling between the pairs of operators $\nabla_{\mu_{2}}, \tau_{k_{I} q_{I}}(\boldsymbol{I})$ and $\tau_{1 \mu_{0}}\left(\boldsymbol{\sigma}_{0}\right), Y_{k q}(\hat{\boldsymbol{R}})$. The form factor $\hat{F}_{k_{I} k E}^{t r i i i}(R)$ is defined as

$$
\begin{aligned}
\hat{F}_{k_{I} k E}^{t r i i i}(R) & =(-)^{3 j_{1}-j_{1}^{\prime}} \hat{J}_{t r}(Q) 15 \sqrt{\frac{3}{2 \pi}} \hat{I}_{\hat{j}_{1}} \hat{j}_{1}^{\prime} \hat{\ell}_{1} \hat{\ell}_{1}^{\prime} u_{\ell_{1} j_{1}}(R) \sum_{k_{I} k_{2} L k E}\left[\hat{g}_{L \ell_{1}^{\prime}} u_{\ell_{1}^{\prime} j_{1}^{\prime}}(R)\right] \\
& \times(-)^{k-L} \hat{L} \hat{k}_{2}^{2}\left(\ell_{1}^{\prime} 010 \mid L 0\right)\left(\ell_{1} 0 L 0 \mid k 0\right) W\left(1 \ell_{1}^{\prime} k \ell_{1} ; L k_{2}\right) \hat{k_{I}} W\left(k_{I} j_{1} I j_{2} ; j_{1}^{\prime} I\right) \\
& \times\left\{\begin{array}{ccc}
j_{1} & j_{1}^{\prime} & k_{I} \\
\frac{1}{2} & \frac{1}{2} & 1 \\
\ell_{1} & \ell_{1}^{\prime} & k_{2}
\end{array}\right\}\left\{\begin{array}{ccc}
1 & k_{I} & E \\
1 & k_{2} & k \\
2 & 1 & 1
\end{array}\right\},
\end{aligned}
$$

and the operator $\boldsymbol{Z}_{k_{I} k E}^{\text {triii }}$ is defined as

$$
\begin{aligned}
\chi_{\mu_{f}}^{*(-)}\left(\boldsymbol{R}, \boldsymbol{k}^{\prime}\right) \boldsymbol{Z}_{k_{I} k E}^{\text {triii }} \chi_{\mu_{i}}^{(+)}(\boldsymbol{R}, \boldsymbol{k})= & \chi_{\mu_{f}}^{*(-)}\left(\boldsymbol{R}, \boldsymbol{k}^{\prime}\right) \sum_{q q_{I} \mu_{0} \mu_{2} \epsilon}\left(1 \mu_{2} k_{I} q_{I} \mid E \epsilon\right) \overleftarrow{\nabla}_{\mu_{2}} \tau_{i_{k_{I} q_{I}}}(\boldsymbol{I})(-1)^{\epsilon} \\
& \times\left(1 \mu_{0} k q \mid E-\epsilon\right) \tau_{1 \mu_{0}}\left(\boldsymbol{\sigma}_{0}\right) Y_{k q}(\hat{\boldsymbol{R}}) \chi_{\mu_{i}}^{(+)}(\boldsymbol{R}, \boldsymbol{k})
\end{aligned}
$$

Performing the sums in Eq. 5.82 with the angular momentum values from the simplified model of ${ }^{10} \mathrm{~B}$ leads to these allowed spin-spin interactions

$$
\begin{aligned}
& \hat{U}_{011}^{t r i i i}(\boldsymbol{R})=\frac{-5 \hat{J}_{t r}(Q)}{12 \sqrt{6 \pi}} u_{1 \frac{3}{2}}(R)\left[\hat{g}_{01}-\hat{g}_{21}\right] u_{1 \frac{3}{2}}(R) \boldsymbol{Z}_{011}^{\text {triii }} \\
& \hat{U}_{110}^{t r i i i}(\boldsymbol{R})=\frac{-\hat{J}_{t r}(Q)}{10 \sqrt{3 \pi}} u_{1 \frac{3}{2}}(R)\left[5 \hat{g}_{01}+11 \hat{g}_{21}\right] u_{1 \frac{3}{2}}(R) \boldsymbol{Z}_{110}^{\text {triii }}, \\
& \hat{U}_{111}^{\text {triii }}(\boldsymbol{R})=\frac{\hat{J}_{t r}(Q)}{30 \sqrt{3} \bar{\pi}} u_{1 \frac{3}{2}}(R)\left[5 \hat{g}_{01}+16 \hat{g}_{21}\right] u_{1 \frac{3}{2}}(R) \boldsymbol{Z}_{111}^{\text {triii }} \\
& \hat{U}_{112}^{t r i i i}(\boldsymbol{R})=\frac{\hat{J}_{t r}(Q)}{50 \sqrt{3 \pi}} u_{1 \frac{3}{2}}(R)\left[10 \hat{g}_{01}-3 \hat{g}_{21}\right] u_{1 \frac{3}{2}}(R) \boldsymbol{Z}_{112}^{\text {triii }}
\end{aligned}
$$




$$
\begin{aligned}
& \hat{U}_{132}^{t r i i i}(\boldsymbol{R})=\frac{-\hat{J}_{t r}(Q)}{25 \sqrt{2 \pi}} u_{1 \frac{3}{2}}(R)\left[\hat{g}_{21} u_{1 \frac{3}{2}}(R)\right] \boldsymbol{Z}_{132}^{\text {triii }}, \\
& \hat{U}_{211}^{t r i i i}(\boldsymbol{R})=\frac{\hat{J}_{t r}(Q)}{3 \sqrt{5 \pi}} u_{1 \frac{3}{2}}(R)\left[\hat{g}_{01}-\hat{g}_{21}\right] u_{1 \frac{3}{2}}(R) \boldsymbol{Z}_{211}^{\text {triii }}, \\
& \hat{U}_{212}^{\text {triii }}(\boldsymbol{R})=\frac{-\hat{J}_{t r}(Q)}{10 \sqrt{\pi}} u_{1 \frac{3}{2}}(R)\left[\hat{g}_{01}-\hat{g}_{21}\right] u_{1 \frac{3}{2}}(R) \boldsymbol{Z}_{212}^{\text {triii }} \\
& \hat{U}_{312}^{\text {triii }}(\boldsymbol{R})=\frac{-\hat{J}_{t r}(Q)}{100 \sqrt{\pi}} u_{1 \frac{3}{2}}(R)\left[5 \hat{g}_{01}+\hat{g}_{21}\right] u_{1 \frac{3}{2}}(R) \boldsymbol{Z}_{312}^{\text {triii }}, \\
& \hat{U}_{332}^{t r i i i}(\boldsymbol{R})=\frac{53 \sqrt{3} \hat{J}_{t r}(Q)}{175 \sqrt{2 \pi}} u_{1 \frac{3}{2}}(R)\left[\hat{g}_{21} u_{1 \frac{3}{2}}(R)\right] \boldsymbol{Z}_{332}^{\text {triii }}, \\
& \hat{U}_{333}^{\text {triii }}(\boldsymbol{R})=\frac{-11 \sqrt{3} \hat{J}_{t r}(Q)}{70 \sqrt{2 \pi}} u_{1 \frac{3}{2}}(R)\left[\hat{g}_{21} u_{1 \frac{3}{2}}(R)\right] \boldsymbol{Z}_{333}^{\text {triii }} \\
& \hat{U}_{334}^{t r i i i}(\boldsymbol{R})=\frac{3 \sqrt{3} \hat{J}_{t r}(Q)}{70 \sqrt{2 \pi}} u_{1 \frac{3}{2}}(R)\left[\hat{g}_{21} u_{1 \frac{3}{2}}(R)\right] \boldsymbol{Z}_{334}^{\text {triii }} .
\end{aligned}
$$

\subsubsection{Spin-Spin Potentials for Tensor Exchange Term iv}

Tensor exchange Term iv will describe the term from Eq. 5.67 in which one grad acts on the initial distorted wave and the other on the initial state valence nucleon wavefunction. Taking this term only in Eq. 5.67 gives

\section{Term iv}

$$
\begin{aligned}
& =(-)^{3 j_{1}-j_{1}^{\prime}-\ell_{1}^{\prime}} \hat{J}_{t r}(Q) 3 \sqrt{6} \hat{I} \hat{j}_{1} \hat{j}_{1}^{\prime} \sum_{\substack{\lambda_{\lambda_{1} \mu_{1} \mu_{2} \mu_{3}} \\
\lambda_{k_{I}}}}\left(1 \mu_{0} 1 \mu_{1} \mid 2-\mu\right)\left(1 \mu_{2} 1 \mu_{3} \mid 2 \mu\right) \tau_{1 \mu_{0}}\left(\boldsymbol{\sigma}_{0}\right) \\
& \times \chi_{\mu_{f}}^{*(-)}\left(\boldsymbol{R}, k^{\prime}\right)\left[\nabla_{\mu_{2}} Y_{\ell_{1} \lambda_{1}}(\hat{\boldsymbol{R}}) u_{\ell_{1} j_{1}}(R)\right] Y_{\ell_{1}^{\prime} \lambda_{1}^{\prime}}^{*}(\hat{\boldsymbol{R}}) u_{\ell_{1}^{\prime} j_{1}^{\prime}}(R)\left[\nabla_{\mu_{3}} \chi_{\mu_{i}}^{(+)}(\boldsymbol{R}, \boldsymbol{k})\right] \\
& \times(-)^{\mu+\mu_{1}-\lambda_{1}} \sum_{k_{I}} \hat{k}_{I}{ }^{2} W\left(k_{I} j_{1} I j_{2} ; j_{1}^{\prime} I\right)\left(I M_{i} k_{I} q_{I} \mid I M_{f}\right) \sum_{k_{2} q_{2}}(-)^{-k_{2}} \\
& \times\left(\ell_{1}-\lambda_{1} \ell_{1}^{\prime} \lambda_{1}^{\prime} \mid k_{2} q_{2}\right)\left(1-\mu_{1} k_{I} q_{I} \mid k_{2} q_{2}\right)\left\{\begin{array}{ccc}
j_{1} & j_{1}^{\prime} & k_{I} \\
\frac{1}{2} & \frac{1}{2} & 1 \\
\ell_{1} & \ell_{1}^{\prime} & k_{2}
\end{array}\right\} .
\end{aligned}
$$

Using the angular momentum coupling techniques analogous to those used in Appendix D.5 for tensor exchange Term C reduces Eq. 5.96 to 


\section{Term iv}

$$
\begin{aligned}
& =(-)^{3 j_{1}-j_{1}^{\prime}} \hat{J}_{t r}(Q) 15 \sqrt{\frac{3}{2 \pi}} \hat{I} \hat{j}_{1} \hat{j}_{1}^{\prime} \hat{\ell}_{1} \hat{\ell}_{1}^{\prime} u_{\ell_{1}^{\prime} j_{1}^{\prime}}(R) \sum_{k_{I} k_{2} L k E}\left[\hat{g}_{L \ell_{1}^{\prime}} u_{\ell_{1}^{\prime} j_{1}^{\prime}}(R)\right] \\
& \times(-)^{k-L-k_{2}+1+k_{I}-E} \hat{L} \hat{k}_{2}^{2}\left(\ell_{1} 010 \mid L 0\right)\left(L 0 \ell_{1}^{\prime} \mid k 0\right) W\left(1 \ell_{1} k \ell_{1}^{\prime} ; L k_{2}\right) \hat{k}_{I} W\left(k_{I} j_{1} I j_{2} ; j_{1}^{\prime} I\right) \\
& \times\left\{\begin{array}{ccc}
j_{1} & j_{1}^{\prime} & k_{I} \\
\frac{1}{2} & \frac{1}{2} & 1 \\
\ell_{1} & \ell_{1}^{\prime} & k_{2}
\end{array}\right\}\left\{\begin{array}{ccc}
1 & k_{I} & E \\
1 & k_{2} & k \\
2 & 1 & 1
\end{array}\right\} \chi_{\mu_{f}}^{*(-)}\left(\boldsymbol{R}, \boldsymbol{k}^{\prime}\right) \sum_{q q_{I} \mu_{0} \mu_{3} \epsilon}\left(1 \mu_{0} k q \mid E \epsilon\right) \tau_{1 \mu_{0}}\left(\boldsymbol{\sigma}_{0}\right) Y_{k q}(\hat{\boldsymbol{R}})(-1)^{\epsilon} \\
& \times\left(k_{I} q_{I} 1 \mu_{3} \mid E-\epsilon\right)\left\langle I, M_{f}\left|\tau_{k_{I} q_{I}}(\boldsymbol{I})\right| I, M_{i}\right\rangle\left[\nabla_{\mu_{3}} \chi_{\mu_{i}}^{(+)}(R, k)\right] .
\end{aligned}
$$

As with tensor exchange Term iii, the folded potentials from Term iv will be denoted by $\hat{U}_{k_{I} k E}^{t r i v}=\hat{F}_{k_{I} k E}^{\text {triv }}(R) \boldsymbol{Z}_{k_{I} k E}^{\text {triv }}$. The extra subscript $E$ represents the coupling between the pairs of operators $\tau_{1 \mu_{0}}\left(\boldsymbol{\sigma}_{0}\right), Y_{k q}(\hat{\boldsymbol{R}})$ and $\tau_{k_{I} q_{I}}(\boldsymbol{I}), \nabla_{\mu_{3}}$. The form factor $\hat{F}_{k_{I} k E}^{\text {triv }}(R)$ is defined as

$$
\begin{aligned}
\hat{F}_{k_{I} k E}^{t r i v}(R) & =(-)^{3 j_{1}-j_{1}^{\prime}} \hat{J}_{t r}(Q) 15 \sqrt{\frac{3}{2 \pi}} \hat{I} \hat{j}_{1} \hat{j}_{1}^{\prime} \hat{\ell}_{1} \hat{\ell}_{1}^{\prime} u_{\ell_{1}^{\prime} j_{1}^{\prime}}(R) \sum_{k_{I} k_{2} L k E}\left[\hat{g}_{L \ell_{1}^{\prime}} u_{\ell_{1}^{\prime} j_{1}^{\prime}}(R)\right] \\
& \times(-)^{k-L-k_{2}+1+k_{I}-E} \hat{L} \hat{k}_{2}^{2}\left(\ell_{1} 010 \mid L 0\right)\left(L 0 \ell_{1}^{\prime} \mid k 0\right) W\left(1 \ell_{1} k \ell_{1}^{\prime} ; L k_{2}\right) \hat{k_{I}} W\left(k_{I} j_{1} I j_{2} ; j_{1}^{\prime} I\right) \\
& \times\left\{\begin{array}{ccc}
j_{1} & j_{1}^{\prime} & k_{I} \\
\frac{1}{2} & \frac{1}{2} & 1 \\
\ell_{1} & \ell_{1}^{\prime} & k_{2}
\end{array}\right\}\left\{\begin{array}{ccc}
1 & k_{I} & E \\
1 & k_{2} & k \\
2 & 1 & 1
\end{array}\right\},
\end{aligned}
$$

and the operator $\boldsymbol{Z}_{k_{I} k E}^{\text {triv }}$ is defined as

$$
\begin{aligned}
\chi_{\mu_{f}}^{*(-)}\left(\boldsymbol{R}, \boldsymbol{k}^{\prime}\right) \boldsymbol{Z}_{k_{I} k E}^{\text {triii }} \chi_{\mu_{i}}^{(+)}\left(\boldsymbol{R}, \boldsymbol{k}_{i}\right) & =\chi_{\mu_{f}}^{*(-)}\left(\boldsymbol{R}, \boldsymbol{k}^{\prime}\right) \sum_{q q_{I} \mu_{0} \mu_{3} \epsilon}\left(1 \mu_{0} k q E \epsilon\right) \tau_{1 \mu_{0}}\left(\boldsymbol{\sigma}_{0}\right) Y_{k q}(\hat{\boldsymbol{R}})(-1)^{\epsilon} \\
& \times\left(k_{I} q_{I} 1 \mu_{3} \mid E-\epsilon\right) \tau_{k_{I} q_{I}}(\boldsymbol{I}) \nabla_{\mu_{3}} \chi_{\mu_{i}}^{(+)}(\boldsymbol{R}, \boldsymbol{k}) .
\end{aligned}
$$

Performing the sums in Eq. 5.97 with the angular momentum values from the simplified model of ${ }^{10} \mathrm{~B}$ leads to these allowed spin-spin interactions

$$
\begin{aligned}
& \hat{U}_{011}^{\text {triii }}(\boldsymbol{R})=\frac{5 \hat{J}_{t r}(Q)}{12 \sqrt{6 \pi}} u_{1 \frac{3}{2}}(R)\left[\hat{g}_{01}-\hat{g}_{21}\right] u_{1 \frac{3}{2}}(R) \boldsymbol{Z}_{011}^{t_{r} i i i}, \\
& \hat{U}_{110}^{t r i i i}(\boldsymbol{R})=\frac{-\hat{J}_{t r}(Q)}{10 \sqrt{3 \pi}} u_{1 \frac{3}{2}}(R)\left[5 \hat{g}_{01}+11 \hat{g}_{21}\right] u_{1 \frac{3}{2}}(R) \boldsymbol{Z}_{110}^{\text {triii }}, \\
& \hat{U}_{111}^{\text {triii }}(\boldsymbol{R})=\frac{-\hat{J}_{t r}(Q)}{30 \sqrt{3 \pi}} u_{1 \frac{3}{2}}(R)\left[5 \hat{g}_{01}+16 \hat{g}_{21}\right] u_{1 \frac{3}{2}}(R) \boldsymbol{Z}_{111}^{\text {triii }}
\end{aligned}
$$




$$
\begin{aligned}
& \hat{U}_{112}^{\text {triii }}(\boldsymbol{R})=\frac{\hat{J}_{t r}(Q)}{50 \sqrt{3 \pi}} u_{1 \frac{3}{2}}(R)\left[10 \hat{g}_{01}-3 \hat{g}_{21}\right] u_{1 \frac{3}{2}}(R) \boldsymbol{Z}_{112}^{\text {triii }} \\
& \hat{U}_{132}^{\text {triii }}(\boldsymbol{R})=\frac{-\hat{J}_{t r}(Q)}{25 \sqrt{2 \pi}} u_{1 \frac{3}{2}}(R)\left[\hat{g}_{21} u_{1 \frac{3}{2}}(R)\right] \boldsymbol{Z}_{132}^{\text {triii }} \\
& \hat{U}_{211}^{\text {triii }}(\boldsymbol{R})=\frac{-\hat{J}_{t r}(Q)}{3 \sqrt{5 \pi}} u_{1 \frac{3}{2}}(R)\left[\hat{g}_{01}-\hat{g}_{21}\right] u_{1 \frac{3}{2}}(R) \boldsymbol{Z}_{211}^{\text {triii }} \\
& \hat{U}_{212}^{\text {triii }}(\boldsymbol{R})=\frac{-\hat{J}_{t r}(Q)}{10 \sqrt{\pi}} u_{1 \frac{3}{2}}(R)\left[\hat{g}_{01}-\hat{g}_{21}\right] u_{1 \frac{3}{2}}(R) \boldsymbol{Z}_{212}^{\text {triii }} \\
& \hat{U}_{312}^{\text {triii }}(\boldsymbol{R})=\frac{-\hat{J}_{t r}(Q)}{100 \sqrt{\pi}} u_{1 \frac{3}{2}}(R)\left[5 \hat{g}_{01}+\hat{g}_{21}\right] u_{1 \frac{3}{2}}(R) \boldsymbol{Z}_{312}^{\text {triii }} \\
& \hat{U}_{332}^{\text {triii }}(\boldsymbol{R})=\frac{53 \sqrt{3} \hat{J}_{t r}(Q)}{175 \sqrt{2 \pi}} u_{1 \frac{3}{2}}(R)\left[\hat{g}_{21} u_{1 \frac{3}{2}}(R)\right] \boldsymbol{Z}_{332}^{\text {trii }} \\
& \hat{U}_{333}^{\text {triii }}(\boldsymbol{R})=\frac{11 \sqrt{3} \hat{J}_{t r}(Q)}{70 \sqrt{2 \pi}} u_{1 \frac{3}{2}}(R)\left[\hat{g}_{21} u_{1 \frac{3}{2}}(R)\right] \boldsymbol{Z}_{333}^{\text {triii }} \\
& \hat{U}_{334}^{\text {triii }}(\boldsymbol{R})=\frac{3 \sqrt{3} \hat{J}_{t r}(Q)}{70 \sqrt{2 \pi}} u_{1 \frac{3}{2}}(R)\left[\hat{g}_{21} u_{1 \frac{3}{2}}(R)\right] \boldsymbol{Z}_{334}^{\text {triii }}
\end{aligned}
$$

By comparing these folded potentials with those from Term iii given in Eqs. 5.85 - 5.95, this simple relationship between the two form factors $\hat{F}_{k_{I} k E}^{t r i i i}(\boldsymbol{R})=(-)^{E} \hat{F}_{k_{I} k E}^{t r i v}(\boldsymbol{R})$ can be determined for this model. This will be useful when examining the time-reversal symmetry of these tensor exchange terms.

\subsubsection{Modified Scattering Amplitudes}

Using the approach detailed in Section 5.4 for the effect of grad operators on distorted wavefunctions and the recoupling techniques detailed in Appendices $\mathrm{A}, \mathrm{C}$ and $\mathrm{D}$ the spin-spin amplitudes for the tensor exchange terms ii, iii and iv are

\section{Term ii}

$$
\begin{aligned}
& f_{k_{I} k}^{t r i i}\left(\mu_{f}, M_{f} ; \mu_{i}, M_{i} ; \theta\right) \\
& \quad=\frac{-45}{E_{c m}} \sqrt{\frac{2}{15}} \hat{k} \sum_{\ell^{\prime}, \ell_{j^{\prime}, j}}(-)^{-j+\mu_{i}+q_{I}} \hat{\ell}^{2} \hat{\ell}^{\prime} \hat{j}^{\prime} \hat{j}^{\prime} \imath^{\ell-\ell^{\prime}} e^{\ell\left(\sigma_{\ell}+\sigma_{\ell^{\prime}}\right)} Y_{\ell^{\prime}, \mu_{i}-q_{I}-\mu_{f}}\left(\hat{\boldsymbol{k}}^{\prime}\right)\left(\ell 0 \frac{1}{2} \mu_{i} \mid j \mu_{i}\right) \\
& \times\left(\ell^{\prime} \mu_{i}-q_{I}-\mu_{f} \frac{1}{2} \mu_{f} \mid j^{\prime} \mu_{i}-q_{I}\right)\left(I M_{i} k_{I} q_{I} \mid I M_{f}\right)\left(j^{\prime} \mu_{i}-q_{I} j-\mu_{i} \mid k_{I}-q_{I}\right) \\
& \quad \times \sum_{L L^{\prime} E}(-)^{-L^{\prime}} \hat{L} \int_{0}^{\infty} R^{2} d R\left[\hat{g}_{L^{\prime} \ell^{\prime}} \frac{u_{\ell^{\prime}, j^{\prime}}\left(k_{c m} R\right)}{R}\right] \hat{F}_{k_{I} k}^{t r i i}(R)\left[\hat{g}_{L \ell} \frac{u_{\ell, j}\left(k_{c m} R\right)}{R}\right] \\
& \quad \times\left(\ell^{\prime} 010 \mid L^{\prime} 0\right)(\ell 010 \mid L 0)\left(k 0 L 0 \mid L^{\prime} 0\right) \hat{E}^{2} W\left(E 2 k_{I} 1 ; k 1\right)\left\{\begin{array}{ccc}
\ell^{\prime} & \ell & E \\
1 & 1 & 2 \\
L^{\prime} & L & k
\end{array}\right\}\left\{\begin{array}{ccc}
\ell^{\prime} & \ell & E \\
\frac{1}{2} & \frac{1}{2} & 1 \\
j^{\prime} & j & k_{I}
\end{array}\right\} .
\end{aligned}
$$




\section{Term iii}

$$
\begin{aligned}
& f_{k_{I} k}^{\text {triii }}\left(\mu_{f}, M_{f} ; \mu_{i}, M_{i} ; \theta\right) \\
& \quad=\frac{\sqrt{6}}{E_{c m}} \hat{k_{I}} \hat{k} \sum_{\ell^{\prime}, \ell, j^{\prime}, j}(-)^{k_{I}+k-\ell-2 j^{\prime}} \hat{\ell}^{2} \hat{\ell}^{\prime} \hat{j}^{\prime} \ell^{\ell-\ell^{\prime}} e^{\imath\left(\sigma_{\ell}+\sigma_{\ell^{\prime}}\right)} Y_{\ell^{\prime}, \mu_{i}-q_{I}-\mu_{f}}\left(\hat{\boldsymbol{k}^{\prime}}\right)\left(\ell 0 \frac{1}{2} \mu_{i} \mid j \mu_{i}\right) \\
& \quad \times\left(\ell^{\prime} \mu_{i}-q_{I}-\mu_{f} \frac{1}{2} \mu_{f} \mid j^{\prime} \mu_{i}-q_{I}\right)\left(I M_{i} k_{I} q_{I} \mid I M_{f}\right)\left(k_{I} q_{I} j^{\prime} \mu_{i}-q_{I} \mid j \mu_{i}\right) \\
& \quad \times \sum_{E L} \hat{E}^{2}(-)^{-L} \int_{0}^{\infty} R d R\left[\hat{g}_{L \ell^{\prime}} \frac{u_{\ell^{\prime}, j^{\prime}}\left(k_{c m} R\right)}{R}\right] \hat{F}_{k_{I} k E}^{t r i i i}(R) u_{\ell, j}\left(k_{c m} R\right)\left(\ell^{\prime} 010 \mid L 0\right) \\
& \quad \times(k 0 \ell 0 \mid L 0) \sum_{E^{\prime}} \hat{E}^{\prime 2} W\left(\frac{1}{2} 1 j^{\prime} \ell^{\prime}, \frac{1}{2} E^{\prime}\right) W\left(k_{I} E^{\prime} j \frac{1}{2} ; \ell j^{\prime}\right)\left\{\begin{array}{ccc}
1 & \ell^{\prime} & E^{\prime} \\
E & 1 & k_{I} \\
k & L & \ell
\end{array}\right\} .
\end{aligned}
$$

\section{Term iv}

$$
\begin{aligned}
& f_{k_{I} k}^{\text {triv }}\left(\mu_{f}, M_{f} ; \mu_{i}, M_{i} ; \theta\right) \\
& =\frac{\sqrt{6}}{E_{c m}} \hat{k_{I}} \hat{k} \sum_{\ell^{\prime}, \ell, j^{\prime}, j}(-)^{\ell-k_{I}-2 j-q_{I}} \hat{\ell}^{2} \hat{j}^{\ell-\ell^{\prime}} e^{\imath\left(\sigma_{\ell}+\sigma_{\ell^{\prime}}\right)} Y_{\ell^{\prime}, \mu_{i}-q_{I}-\mu_{f}}\left(\hat{\boldsymbol{k}}^{\prime}\right)\left(\ell 0 \frac{1}{2} \mu_{i} \mid j \mu_{i}\right) \\
& \quad \times\left(\ell^{\prime} \mu_{i}-q_{I}-\mu_{f} \frac{1}{2} \mu_{f} \mid j^{\prime} \mu_{i}-q_{I}\right)\left(I M_{i} k_{I} q_{I} \mid I M_{f}\right)\left(k_{I}-q_{I} j \mu_{i} \mid j^{\prime} \mu_{i}-q_{I}\right) \\
& \quad \times \sum_{E L} \hat{L} \hat{E}^{2}(-)^{E-L} \int_{0}^{\infty} R d R u_{\ell^{\prime}, j^{\prime}}\left(k_{c m} R\right) \hat{F}_{k_{I} k E}^{t r i v}(R)\left[\hat{g}_{L \ell} \frac{u_{\ell, j}\left(k_{c m} R\right)}{R}\right](\ell 010 \mid L 0) \\
& \quad \times\left(k 0 L 0 \mid \ell^{\prime} 0\right) \sum_{E^{\prime}} \hat{E}^{\prime 2} W\left(\frac{1}{2} 1 j \ell, \frac{1}{2} E^{\prime}\right) W\left(k_{I} E^{\prime} j^{\prime} \frac{1}{2} ; \ell^{\prime} j\right)\left\{\begin{array}{ccc}
1 & E^{\prime} \\
E & 1 & k_{I} \\
k & L & \ell^{\prime}
\end{array}\right\} .
\end{aligned}
$$

Note that $f_{k_{I} k}^{t r i}\left(\mu_{f}, M_{f} ; \mu_{i}, M_{i} ; \theta\right)$ is calculated using Eq. 2.27. For all of these scattering amplitudes the $\mathrm{z}$-axis is taken to be along the incident beam direction and the $\mathrm{y}$-axis chosen to be perpendicular to the scattering plane.

The scattering amplitude for the whole tensor exchange term in the NN interaction is given by

$$
f_{k_{I} k}^{t r E x}\left(\mu_{f}, M_{f} ; \mu_{i}, M_{i} ; \theta\right)=f_{k_{I} k}^{t r i}+f_{k_{I} k}^{t r i i}+f_{k_{I} k}^{t r i i}+f_{k_{I} k}^{t r i v}
$$

\subsubsection{Time-Reversal Invariance}

Time reversal reverses the direction of rotation of spins (i.e. changes the sign of their projections) and inverts the direction of momenta. Taking $\boldsymbol{\Theta}$ to be the time reversal operator as in [44], its affect on a particle with spin $I$, projection $M$ and momentum $\boldsymbol{k}$ is

$$
\Theta|k ; I M\rangle=(-)^{I-M}|-\boldsymbol{k} ; I-M\rangle \text {. }
$$


The sign and inclusion $I$ in the phase change in Eq. 5.115 is a convention taken from [44].

The explicit expression that relates scattering amplitudes for inverse reactions is given in [44]

$\left\langle\boldsymbol{k}^{\prime} ; \frac{1}{2} \mu_{f}, I M_{f}|f(\theta, \phi)| \boldsymbol{k} ; \frac{1}{2} \mu_{i}, I M_{i}\right\rangle=(-)^{\eta_{f i}}\left\langle-\boldsymbol{k} ; \frac{1}{2}-\mu_{i}, I-M_{i}|f(\theta, \phi)|-\boldsymbol{k}^{\prime} ; \frac{1}{2}-\mu_{f}, I-M_{f}\right\rangle$,

where

$$
\eta_{f i}=\frac{1}{2}-\mu_{f}+I-M_{f}+\frac{1}{2}-\mu_{i}+I-M_{i} .
$$

Applying this to each term on the right hand side of Eq. 5.114 results in

$$
\begin{aligned}
& \left\langle-\boldsymbol{k} ; \frac{1}{2}-\mu_{i}, I-M_{i}\left|f_{k_{I} k}^{t r i}(\theta)\right| \boldsymbol{k} ; \frac{1}{2} \mu_{i}, I M_{i}\right\rangle=(-)^{1+k_{I}+\eta_{f i}}\left\langle\boldsymbol{k}^{\prime} ; \frac{1}{2} \mu_{f}, I M_{f}\left|f_{k_{I} k}^{t r i}(\theta)\right| \boldsymbol{k} ; \frac{1}{2} \mu_{i}, I M_{i}\right\rangle \\
& \left\langle-\boldsymbol{k} ; \frac{1}{2}-\mu_{i}, I-M_{i}\left|f_{k_{I} k}^{t r i i}(\theta)\right| \boldsymbol{k} ; \frac{1}{2} \mu_{i}, I M_{i}\right\rangle=(-)^{1+k_{I}+\eta_{f i}}\left\langle\boldsymbol{k}^{\prime} ; \frac{1}{2} \mu_{f}, I M_{f}\left|f_{k_{I} k}^{t r i i}(\theta)\right| \boldsymbol{k} ; \frac{1}{2} \mu_{i}, I M_{i}\right\rangle, \\
& \left\langle-\boldsymbol{k} ; \frac{1}{2}-\mu_{i}, I-M_{i}\left|f_{k_{I} k}^{t r i i i}(\theta)\right| \boldsymbol{k} ; \frac{1}{2} \mu_{i}, I M_{i}\right\rangle=(-)^{\eta_{f i}}\left\langle\boldsymbol{k}^{\prime} ; \frac{1}{2} \mu_{f}, I M_{f}\left|f_{k_{I} k}^{t r i v}(\theta)\right| \boldsymbol{k} ; \frac{1}{2} \mu_{i}, I M_{i}\right\rangle \\
& \left\langle-\boldsymbol{k} ; \frac{1}{2}-\mu_{i}, I-M_{i}\left|f_{k_{I} k}^{t r i v}(\theta)\right| \boldsymbol{k} ; \frac{1}{2} \mu_{i}, I M_{i}\right\rangle=(-)^{\eta_{f i}}\left\langle\boldsymbol{k}^{\prime} ; \frac{1}{2} \mu_{f}, I M_{f}\left|f_{k_{I} k}^{t r i i i}(\theta)\right| \boldsymbol{k} ; \frac{1}{2} \mu_{i}, I M_{i}\right\rangle .
\end{aligned}
$$

In order to obtain the expressions in Eqs. 5.120 and 5.121 the relation $\hat{F}_{k_{K} k E}^{\text {triii }}(\boldsymbol{R})=$ $(-)^{E} \hat{F}_{k_{I} k E}^{t r i v}(\boldsymbol{R})$ discussed in Section 5.5.6 has been used.

In Eqs. 5.118 and 5.119, the phase factor $(-)^{1+k_{I}}$ has been left in as it does not cancel under time reversal. However, when evaluating the folded potentials for these amplitudes only terms with odd values of $k_{I}$ survive (see Sections 5.5.3 and 5.5.4), so $(-)^{1+k_{I}}=1$. Eqs. 5.120 and 5.121 do not have this factor so even though they do have folded potentials with even values of $k_{I}$ they do not break time reversal symmetry. 


\section{Aide Memoire}

Now that all the folding formalism has been defined for the direct (Chapter 3) and exchange (Chapter 5) terms, a brief summary will be given here to aid the reading of the next chapter. In total four terms in the NN interaction have been folded over the valence nucleon wavefunction to give spin-spin interactions. These four terms are

$\left\langle\Psi_{I, M_{f}}\left(\boldsymbol{r}_{1}, \boldsymbol{r}_{2}\right)\left|v_{N N}^{\sigma \sigma}(r) \boldsymbol{\sigma}_{a} \cdot \boldsymbol{\sigma}_{b}+\hat{v}_{N N}^{\sigma \sigma}(r) \boldsymbol{\sigma}_{a} \cdot \boldsymbol{\sigma}_{b} P_{a b}^{r}+v_{N N}^{t r}(r) \boldsymbol{S}_{a b}+\hat{v}_{N N}^{t r}(r) \boldsymbol{S}_{a b} P_{a b}^{r}\right| \Psi_{I, M_{i}}\left(\boldsymbol{r}_{1}^{\prime}, \boldsymbol{r}_{2}^{\prime}\right)\right\rangle$,

where the NN interaction is between projectile nucleon $a$ and valence nucleon $b$ with separation $r$ and the hats denote exchange terms.

For protons elastically scattered from ${ }^{10} \mathrm{~B}$ these result in numerous local and non-local spin-spin interactions. The notation for each type is summarised in Table AM.

\begin{tabular}{|c|c|c|}
\hline $\begin{array}{c}\text { NN Interaction } \\
\text { Term }\end{array}$ & $\begin{array}{c}\text { Notation for } \\
\text { Spin-Spin Potentials }\end{array}$ & $\begin{array}{c}\text { Form of } \\
\text { Spin-Spin Potentials }\end{array}$ \\
\hline $\begin{array}{l}\text { Central Direct } \\
v_{N N}^{\sigma \sigma}(r) \boldsymbol{\sigma}_{a} \cdot \boldsymbol{\sigma}_{b} \\
\end{array}$ & $U_{10}^{\sigma \sigma}(R), U_{12}^{\sigma \sigma}(\boldsymbol{R}), U_{32}^{\sigma \sigma}(\boldsymbol{R})$ & $U_{k_{I} k}^{\sigma \sigma}(\boldsymbol{R})=F_{k_{I} k}^{\sigma \sigma}(R) \boldsymbol{S}_{k_{I} k}$ \\
\hline $\begin{array}{l}\text { Central Exchange } \\
\hat{v}_{N N}^{\sigma \sigma}(r) \boldsymbol{\sigma}_{a} \cdot \boldsymbol{\sigma}_{b} P_{a b}^{r}\end{array}$ & $\hat{U}_{10}^{\sigma \sigma}(R), \hat{U}_{12}^{\sigma \sigma}(\boldsymbol{R}), \hat{U}_{32}^{\sigma \sigma}(\boldsymbol{R})$ & $\hat{U}_{k_{I} k}^{\sigma \sigma}(\boldsymbol{R})=\hat{F}_{k_{I} k}^{\sigma \sigma}(R) \boldsymbol{S}_{k_{I} k}$ \\
\hline $\begin{array}{c}\text { Tensor Direct } \\
v_{N N}^{t r}(r) \boldsymbol{S}_{a b}\end{array}$ & $U_{10}^{t r}(R), U_{12}^{t r}(\boldsymbol{R}), U_{32}^{t r}(\boldsymbol{R}), U_{34}^{t r}(\boldsymbol{R})$ & $U_{k_{I} k}^{t r}(\boldsymbol{R})=F_{k_{I} k}^{t r}(R) \boldsymbol{S}_{k_{I} k}$ \\
\hline $\begin{array}{c}\text { Tensor Exchange } \\
\hat{v}_{N N}^{t r}(r) \boldsymbol{S}_{a b} P_{a b}^{r}\end{array}$ & 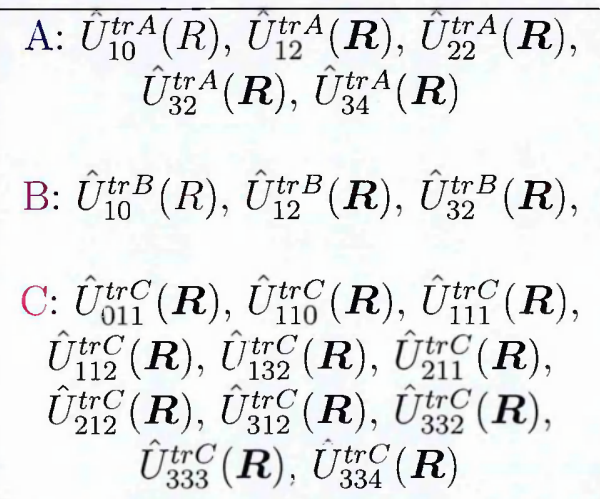 & $\begin{array}{c}\hat{U}_{k_{I} k}^{\operatorname{tr} A}(\boldsymbol{R})=\hat{F}_{k_{I} k}^{t r A}(R) \boldsymbol{S}_{k_{I} k} \\
\hat{U}_{k_{I} k}^{t r B}(\boldsymbol{R})=\hat{F}_{k_{I} k}^{\operatorname{tr} B}(R) \boldsymbol{Y}_{k_{I} k}^{\operatorname{tr} B} \\
\hat{U}_{k_{I} k E}^{t r C}(\boldsymbol{R})=\hat{F}_{k_{I} k E}^{t r C}(R) \boldsymbol{Z}_{k_{I} k E}^{t r C}\end{array}$ \\
\hline
\end{tabular}

Table AM: Summary of notation for spin-spin potentials. 
Where operators $\boldsymbol{Y}_{k_{I} k}^{t r B}$ and $\boldsymbol{Z}_{k_{I} k E}^{t r C}$ act on the initial distorted wavefunction and are given by

$$
\begin{aligned}
\boldsymbol{Y}_{k_{I} k}^{t r B} & =\left[Y_{k}(\hat{\boldsymbol{R}}) \times \tau_{k_{I}}(\boldsymbol{I})\right] \cdot\left[\tau_{1}\left(\boldsymbol{\sigma}_{0}\right) \times \mathscr{Y}_{2}\left(-\imath \nabla_{\boldsymbol{R}}\right)\right], \\
\boldsymbol{Z}_{k_{I} k E}^{t r C} & =\left[Y_{k}(\hat{\boldsymbol{R}}) \times \tau_{1}\left(\boldsymbol{\sigma}_{0}\right)\right]_{E \epsilon}\left[\tau_{k_{I}}(\boldsymbol{I}) \times \nabla\right]_{E-\epsilon} .
\end{aligned}
$$




\section{Chapter 6}

\section{Spin-spin Interactions and Observables}

In Chapters 3 and 5 the folding formalism was developed for spin-spin interactions within a two valence-particle model for the target nucleus. The details of the effective NN interaction used in the folding model, both direct and exchange terms, were given in Chapter 4. These components will now be used to calculate the spin-spin potentials for $200 \mathrm{MeV}$ protons elastically scattered from ${ }^{10} \mathrm{~B}$. These spin-spin interactions will then be used to calculate the scattering amplitudes, as described in Chapters 2 and 5 , and hence the polarisation transfer coefficient $D_{N N}$.

\subsection{Single-Particle Wavefunction}

In order to calculate the spin-spin interactions detailed in Chapters 3 and 5 the singleparticle wavefunction for the valence proton and neutron in the $1 p_{\frac{3}{2}}$ shell of the ${ }^{10} \mathrm{~B}$ nucleus must be chosen. To investigate the sensitivity of $D_{N N}$ to the shape of the radial wavefunction two different types will be used.

\section{Harmonic Oscillator}

Harmonic oscillator (HO) wavefunctions can be a reasonable approximation for low lying bound states [91] and have the advantage that they can be integrated and differentiated analytically. The radial function (from Eq. 3.15) for a single-particle in the $1 p$ level of a $\mathrm{HO}$ potential well is given by

$$
u_{1 \frac{3}{2}}(r)=\sqrt{\frac{8(2 \nu)^{5 / 2}}{3 \sqrt{\pi}}} r e^{-\nu r^{2}}
$$


the parameter $\nu$ is defined by

$$
\nu=\frac{m \omega}{2 \hbar}
$$

where $m$ is the mass of the particle and $\omega$ is the angular frequency of the oscillator [91]. The value of $\nu=0.1966 \mathrm{fm}^{-2}$ used in this work is taken from ref [92] in which the harmonic oscillator constant was calculated for different $p$ shell nuclei using Coulomb energy data. The resulting radial function is shown by the black curve in Figure 6.1.

\section{Woods-Saxon}

The other type of single-particle wavefunction used in this work is one calculated from a Woods-Saxon (WS) potential and will be referred to here as a 'WS wavefunction'. This wavefunction is calculated using a binding energy of $7.0 \mathrm{MeV}$ and a WS potential with parameters $R_{W S}=(0.88 \pm 0.13)(A-1)^{1 / 3} \mathrm{fm}$ and $a=0.81 \pm 0.08 \mathrm{fm}$ for the diffuseness. These parameters are taken from ref [93], who obtain the $1 p_{\frac{3}{2}}$ wavefunction from the transform of the electron scattering form factor of the $1.740 \mathrm{MeV}$ excited state in ${ }^{10} \mathrm{~B}$.

The single-particle wavefunction of the valence proton incorporates the effect of the Coulomb interaction with the core making it slightly different to the neutron wavefunction. The radial functions obtained from the Woods-Saxon potential parameters for the valence proton (red curve) and valence neutron (blue curve) are also shown in Figure 6.1.

The differences between the HO and WS wavefunctions in Figure 6.1 are quite subtle. The peaks of the three curves are of similar heights and at similar values of $r$. The most

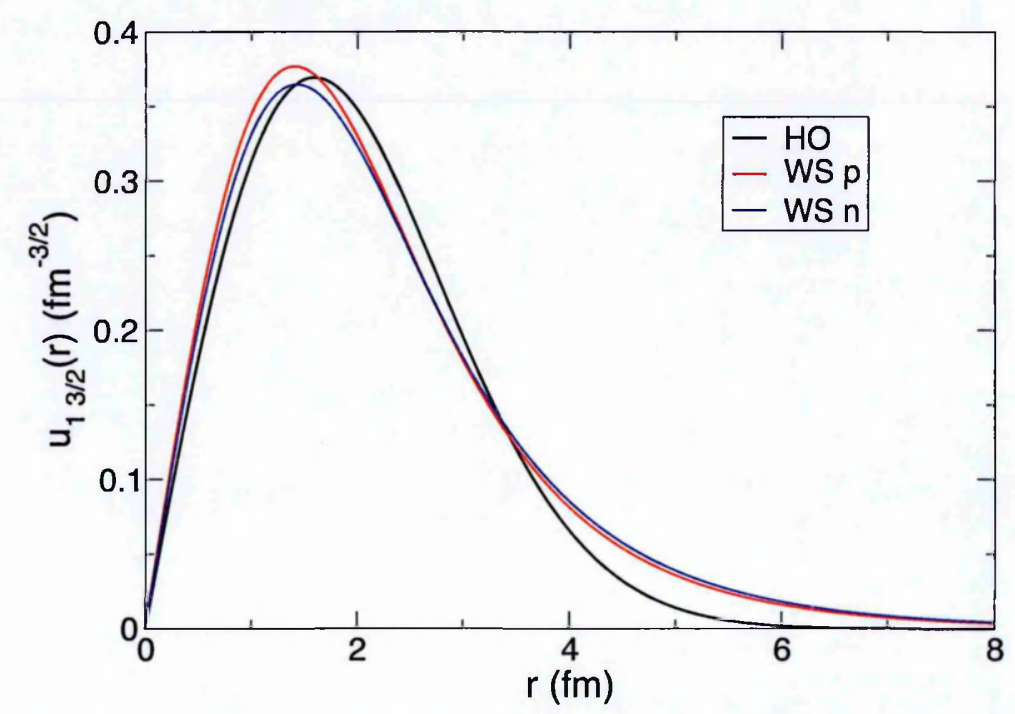

Figure 6.1: The $1 p_{\frac{3}{2}}$ single-particle wavefunctions. The black curve is the harmonic oscillator wavefunction for $\nu=0.1966 \mathrm{fm}^{-2}$. The red and blue curves are the WS wavefunctions for the valence proton and neutron respectively. 
significant difference is that the WS wavefunctions decrease less rapidly than the HO wavefunction at large $r$. Throughout this chapter the effect of using these two different types of wavefunctions on the spin-spin interactions and the calculation of $D_{N N}$ will be examined.

\subsection{Direct Spin-Spin Interactions}

Chapter 3 describes the folding of the direct terms in the NN interaction to give spinspin interactions of the form $U_{k_{I} k}(\boldsymbol{R})=F_{k_{I} k}(R) \boldsymbol{S}_{k_{I} k}$. The form factors $F_{k_{I} k}^{\sigma \sigma}(R)$ from the folding of the central term $v_{p p}^{\sigma \sigma}\left(r_{p}\right) \boldsymbol{\sigma}_{0} \cdot \boldsymbol{\sigma}_{1}+v_{p n}^{\sigma \sigma}\left(r_{n}\right) \boldsymbol{\sigma}_{0} \cdot \boldsymbol{\sigma}_{2}$ are given in Eqs. 3.31 to 3.33 for protons elastically scattered from ${ }^{10} \mathrm{~B}$. These potentials are plotted in Figure 6.2 using the two different types of single-particle wavefunction discussed in the previous section. The volume integrals of these form factors can be used to check the folding formalism as discussed in Section 3.9.1. The numbers obtained from this check are given in Table 6.1.

As is noted in the early works on spin-spin interactions [19], the spherical $F_{10}^{\sigma \sigma}(R)$ potential is the strongest. In agreement with McAbee [20,21], the $F_{32}^{\sigma \sigma}(R)$ potential is found to be larger than the tensor $F_{12}^{\sigma \sigma}(R)$ potential. At their peaks the magnitudes of the form factor $F_{32}^{\sigma \sigma}(R)$ is approximately $3 \times F_{12}^{\sigma \sigma}(R)$, but before McAbee the $U_{32}(\boldsymbol{R})$ was neglected from all calculations using spin-spin interactions.

There is not a significant difference between the spin-spin interactions calculated from the HO (Figure 6.2(a)) and the WS (Figure 6.2(b)) wavefunctions. Those calculated using the latter are slightly stronger but there is no effect on the shape of the potentials.

The spin-spin form factors from the folding of the direct tensor term $v_{p p}^{t r}\left(r_{p}\right) \boldsymbol{S}_{01}+$ $v_{p n}^{t r}\left(r_{n}\right) \boldsymbol{S}_{02}$ in the NN interaction are given in Eqs. 3.54 to 3.57 for proton elastic scattering from ${ }^{10} \mathrm{~B}$. These potentials are plotted in Figure 6.3 using the two different single-particle wavefunctions. The volume integrals of these form factors can be used to check the folding formalism as discussed in Section 3.9.2. The numbers obtained from this check are given

\begin{tabular}{|c||c|c|c|}
\hline$k_{I}, k$ & Eq. no. & Wfn & Result \\
\hline \hline 1,0 & 3.62 & $\begin{array}{l}\text { HO } \\
\text { WS }\end{array}$ & $\begin{array}{c}-801.234+502.983 \imath \mathrm{MeV} . \mathrm{fm}^{3} \\
-801.234+502.983 \imath \mathrm{MeV} . \mathrm{fm}^{3}\end{array}$ \\
\hline 1,2 & 3.68 & $\begin{array}{c}\text { HO } \\
\text { WS }\end{array}$ & $\begin{array}{c}-51.278+32.191 \imath \mathrm{MeV} . \mathrm{fm}^{5} \\
-58.392+38.772 \imath \mathrm{MeV} . \mathrm{fm}^{5}\end{array}$ \\
\hline 3,2 & 3.68 & $\begin{array}{c}\text { HO } \\
\text { WS }\end{array}$ & $\begin{array}{c}133.226-83.634 \imath \mathrm{MeV} . \mathrm{fm}^{5} \\
154.306-100.732 \imath \mathrm{MeV} . \mathrm{fm}^{5}\end{array}$ \\
\hline
\end{tabular}

Table 6.1: The volume integrals, as defined in Section 3.9.1, of the spin-spin interactions calculated from the folding of the direct central term in the NN interaction. 


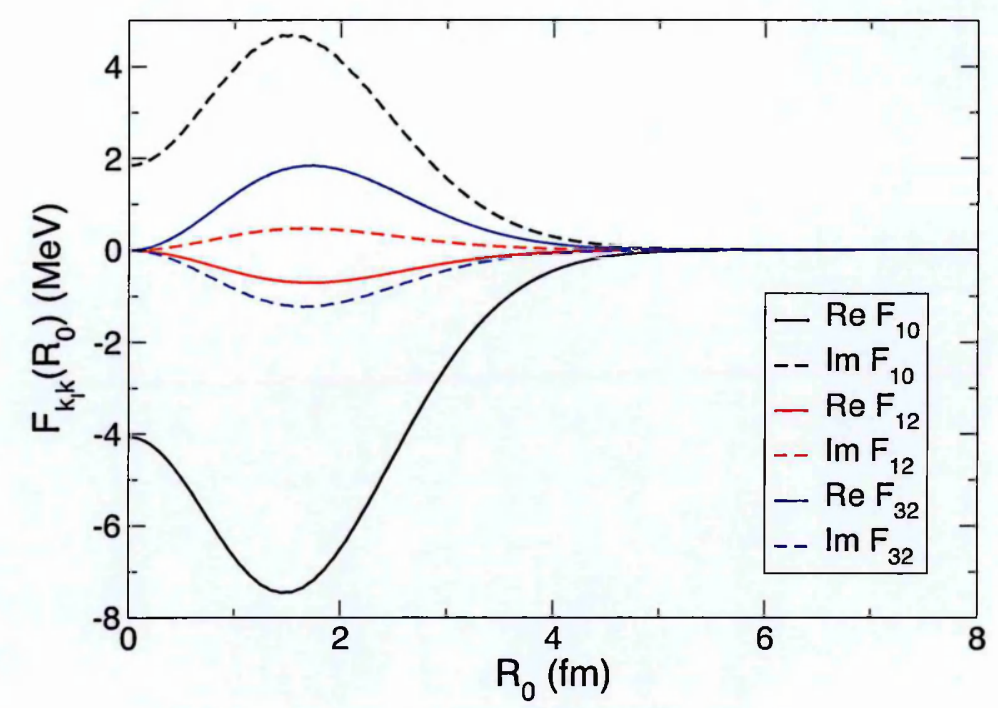

(a) Harmonic oscillator wavefunction

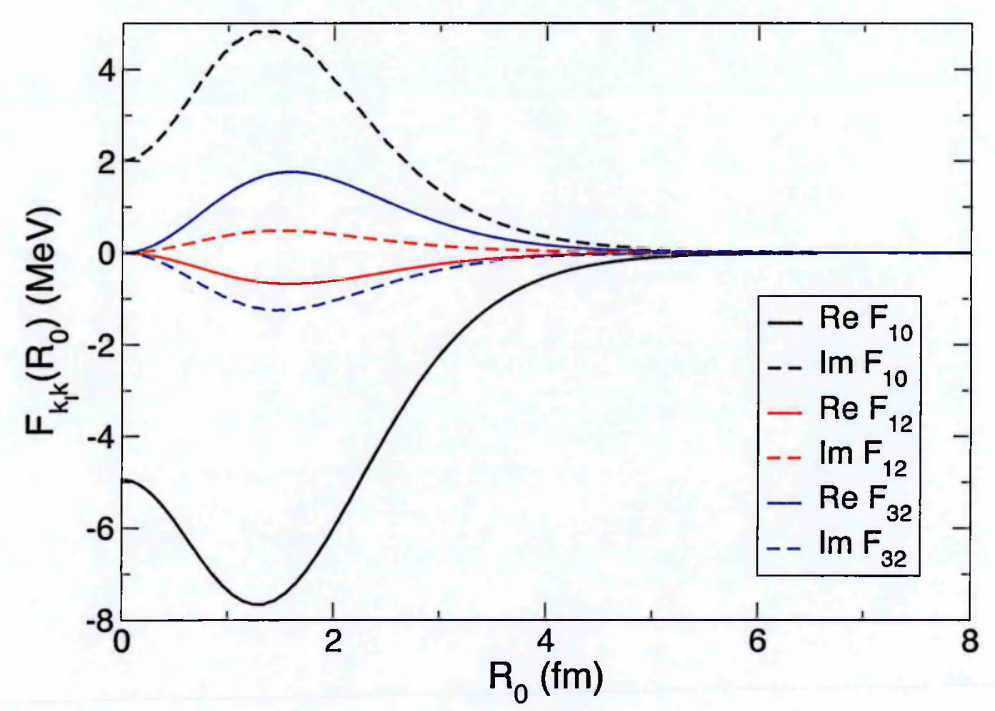

(b) Woods-Saxon wavefunction

Figure 6.2: Spin-spin interactions calculated from the folding of the direct central $v_{p p}^{\sigma \sigma}\left(r_{p}\right) \sigma_{0} \cdot \sigma_{1}+v_{p n}^{\sigma \sigma}\left(r_{n}\right) \sigma_{0} \cdot \sigma_{2}$ term in the NN interaction, using (a) HO wavefunction and (b) WS wavefunction, for elastic proton scattering from ${ }^{10} \mathrm{~B}$.

in Table 6.2.

The spin-spin potentials in Figure 6.3 have more radial structure than those in Figure 6.2 because they are a superposition of three different terms (see Section 3.6). The spherical $F_{10}^{t r}(R)$ potential is again shown to be significantly stronger than the others, however the $F_{32}^{\operatorname{tr}}(R)$ interaction is weaker than $F_{12}^{\operatorname{tr}}(R)$ in contrast to the same rank form factors from the $v_{N N}^{\sigma \sigma}(r) \sigma_{a} \cdot \sigma_{b}$ folding. The new spin-spin term, $F_{34}^{t r}(R)$, which goes beyond the work of McAbee is also stronger than the $F_{32}^{t r}(R)$ potential and comparable in magnitude to $F_{12}^{t r}(R)$. 


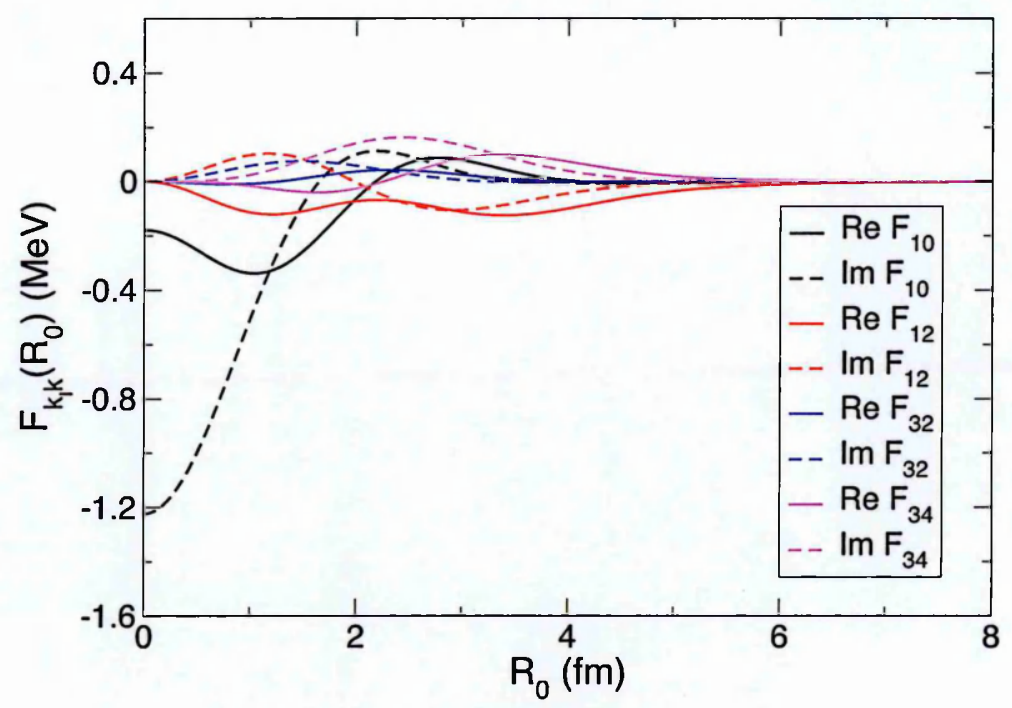

(a) Harmonic oscillator wavefunction

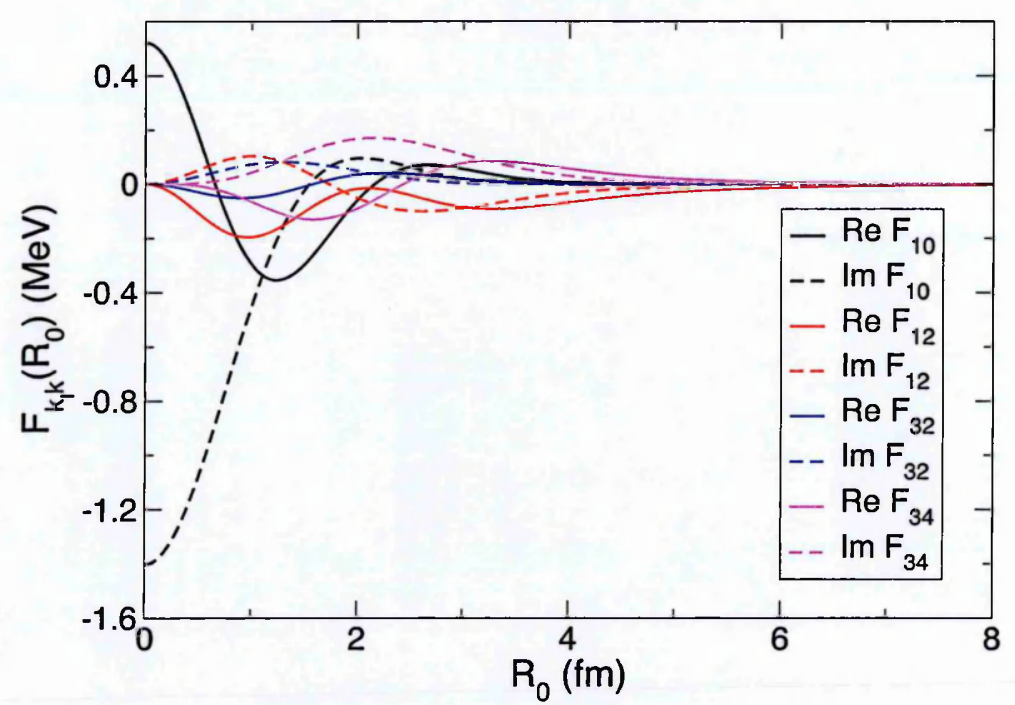

(b) Woods-Saxon wavefunction

Figure 6.3: Spin-spin interactions calculated from the folding of the direct tensor $v_{p p}^{t r}\left(r_{p}\right) \boldsymbol{S}_{01}+v_{p n}^{t r}\left(r_{n}\right) \boldsymbol{S}_{02}$ term in the NN interaction, using (a) HO wavefunction and (b) WS wavefunction, for elastic proton scattering from ${ }^{10} \mathrm{~B}$.

These spin-spin potentials reveal more of a difference between the calculations using the HO and WS single-particle wavefunctions. The most significant difference can be seen between the real parts of the $F_{10}^{t r}(R)$ form factors. At $R=0$ the value of $F_{10}^{t r}(R)$ changes from $-0.178 \mathrm{MeV}$ for $\mathrm{HO}$ to $0.522 \mathrm{MeV}$ for WS. To see if differences caused by the radial wavefunctions also significantly affect the scattering observables a simple calculation of $D_{N N}$ will be performed which limits the inclusion of spin-spin interactions to only the spherical term $U_{10}(R)$. 


\begin{tabular}{|c||c|c|c|}
\hline$k_{I}, k$ & Eq. no. & Wfn & Result \\
\hline \hline 1,0 & \multirow{2}{*}{3.70} & HO & 0 \\
& & WS & 0 \\
\hline 1,2 & \multirow{2}{*}{3.73} & HO & $\begin{array}{c}-99.006-39.247 \imath \mathrm{MeV} . \mathrm{fm}^{5} \\
-99.006-39.247 \imath \mathrm{MeV} . \mathrm{fm}^{5}\end{array}$ \\
\hline 3,2 & \multirow{2}{*}{3.73} & HO & 0 \\
& WS & 0 \\
\hline 3,4 & \multirow{2}{*}{3.79} & HO & $\begin{array}{c}1520.19+602.612 \imath \mathrm{MeV} . \mathrm{fm}^{7} \\
2499.009+780.069 \imath \mathrm{MeV}_{\mathrm{fm}}\end{array}$ \\
\hline
\end{tabular}

Table 6.2: The volume integrals, as defined in Section 3.9.2, of the spin-spin interactions calculated from the folding of the direct tensor term in the NN interaction.

\subsubsection{Spherical Spin-Spin Interaction}

As defined in Chapter 2 the spherical spin-spin interaction has the form

$$
U_{10}(R)=\frac{-F_{10}(R)}{\sqrt{4 \pi} \sqrt{I(I+1)}} \sigma_{0} \cdot \boldsymbol{I}
$$

where for the following discussion $F_{10}(R)=F_{10}^{\sigma \sigma}(R)+F_{10}^{\operatorname{tr}}(R)$.

\section{No Spin-Orbit}

In Section 2.2.1, the DWBA scattering amplitude for the spherical spin-spin interaction is evaluated when the spin-orbit term in the distorting potential is set to zero (see Eq. 2.31). Eq. 2.32 then gives the total nucleon-nucleus elastic scattering amplitude in terms of the spherical spin-spin amplitude, $h_{10}(\theta)$, and $g(\theta)$, the amplitude for a spin-zero projectile and target. Figure 6.4(a) shows $\left|h_{10}(\theta)\right|^{2}$ and $|g(\theta)|^{2}$, the latter without the Coulomb amplitude $f_{\text {Coul }}(\theta)$ shown in Eq. 2.41. Figure 6.4(b) shows the ratio-to-Rutherford cross section calculated from the full $g(\theta)$ amplitude only (black curve) and the whole elastic scattering amplitude given in Eq. 2.32 (red curves), for $200 \mathrm{MeV}$ protons elastically scattered from ${ }^{10} \mathrm{~B}$.

Figure 6.4(a) shows that the spin-zero scattering amplitude $|g(\theta)|^{2}$, without the Coulomb contribution, is approximately two orders of magnitude larger that the spherical spin-spin amplitude $\left|h_{10}(\theta)\right|^{2}$, except close to $40^{\circ}$. In this region the minimum in the spin-zero amplitude and the maximum in spin-spin amplitude means $|g(\theta)|^{2} \approx 3\left|h_{10}(\theta)\right|^{2}$. It is in this region that the effect of the spin-spin interactions on the observables is at a maximum.

Figure 6.4(b) shows that the spherical spin-spin amplitude has little effect on the cross section. The only noticeable difference between the black curve, which has no spin-spin interactions in the calculation, and the red curves, which do include spin-spin effects, is 


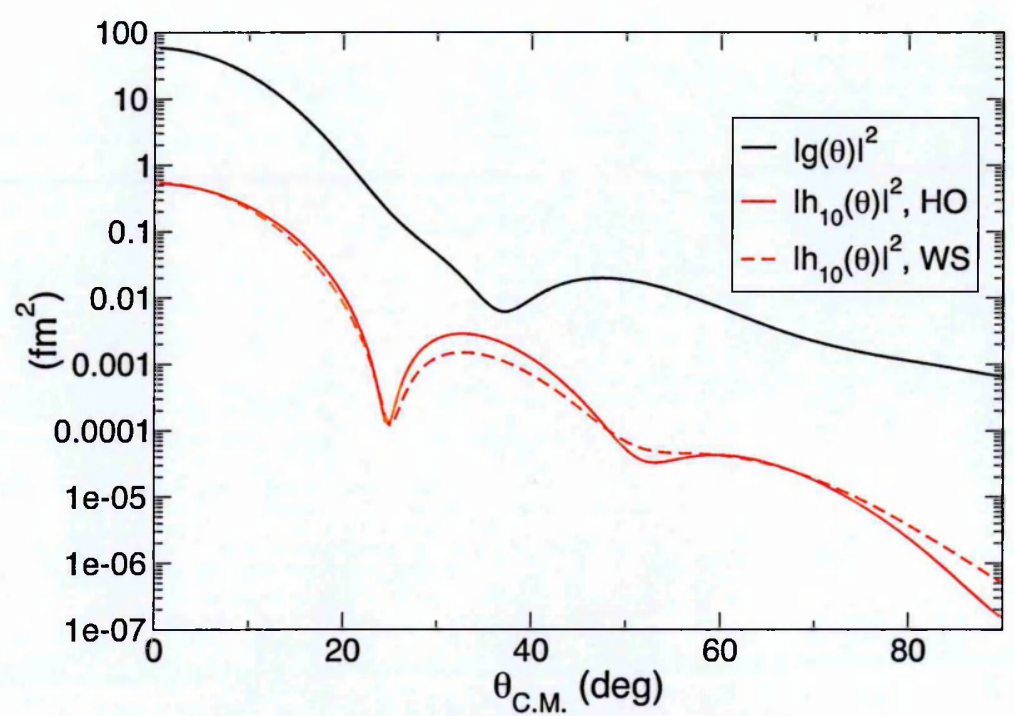

(a) $g(\theta)$ (no Coulomb) and $h_{10}(\theta)$ amplitudes

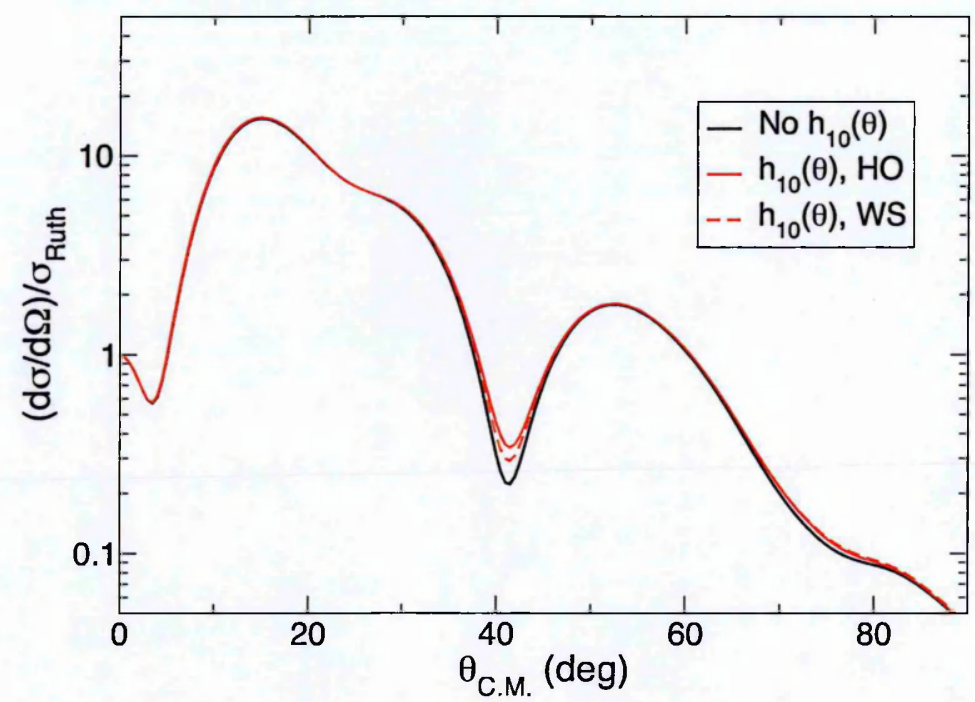

(b) Ratio-to-Rutherford cross section

Figure 6.4: (a) Spin-zero, $|g(\theta)|^{2}$ (without Coulomb contribution) and spherical spin-spin, $\left|h_{10}(\theta)\right|^{2}$ modulus squared scattering amplitudes for elastic proton scattering from ${ }^{10} \mathrm{~B}$. (b) ratio-to-Rutherford cross section excluding (black curve) and including (red curves) the $h_{10}(\theta)$ amplitude for elastic proton scattering from ${ }^{10} \mathrm{~B}$. In both figures the amplitudes were calculated without spin-orbit distorting potential and $h_{10}(\theta)$ was calculated with both HO (solid red line) and WS (dashed red line) single-particle wavefunctions. 
at the cross section minimum at $\sim 40^{\circ}$. Here the spin-spin amplitude slightly fills in the minimum. The cross section data for elastic protons scattering from ${ }^{10} \mathrm{~B}$ is given in Fig. 3 of [35]. It differs significantly from the calculation given in Fig. 6.4(b). For a better comparison, inclusion of the spin-orbit term in the distorting potential is needed. This will be examined in the next section.

The calculation of the polarisation transfer coefficient $D_{N N}$, given in Eq. 2.33, is shown in Figure 6.5. It can clearly be seen from Fig. 6.5 that the deviation of $D_{N N}$ from unity is most significant in the region around $40^{\circ}$. This is where the value of $\left|h_{10}(\theta)\right|^{2}$ is closest to the full $|g(\theta)|^{2}$, including Coulomb contributions, and where the second minimum occurs in the cross section. At $\sim 40^{\circ}$ the effect of the spin-spin interactions is at a maximum compared to the standard terms in the optical potential, which is why it is at this angle that $D_{N N}$ has its greatest deviation from unity.

To see how the deviation of $D_{N N}$ from unity is related to the strength of the spinspin interaction the ratio of $1-D_{N N}$ for $F_{10}(R)$ and $0.5 \times F_{10}(R)$ is plotted in Figure 6.6. It shows quite clearly that when the strength of the spin-spin potential changes by a factor of 2 the deviation of $D_{N N}$ from 1 changes by a factor of 4 . This confirms the quadratic relationship between $1-D_{N N}$ and $h_{10}(\theta)$ given in Eq. 2.33. It also indicates that to further investigate spin-spin interactions it would be better to study an observable with a first order dependence on the strengths of the spin-spin interactions. This will be discussed further in the next chapter.

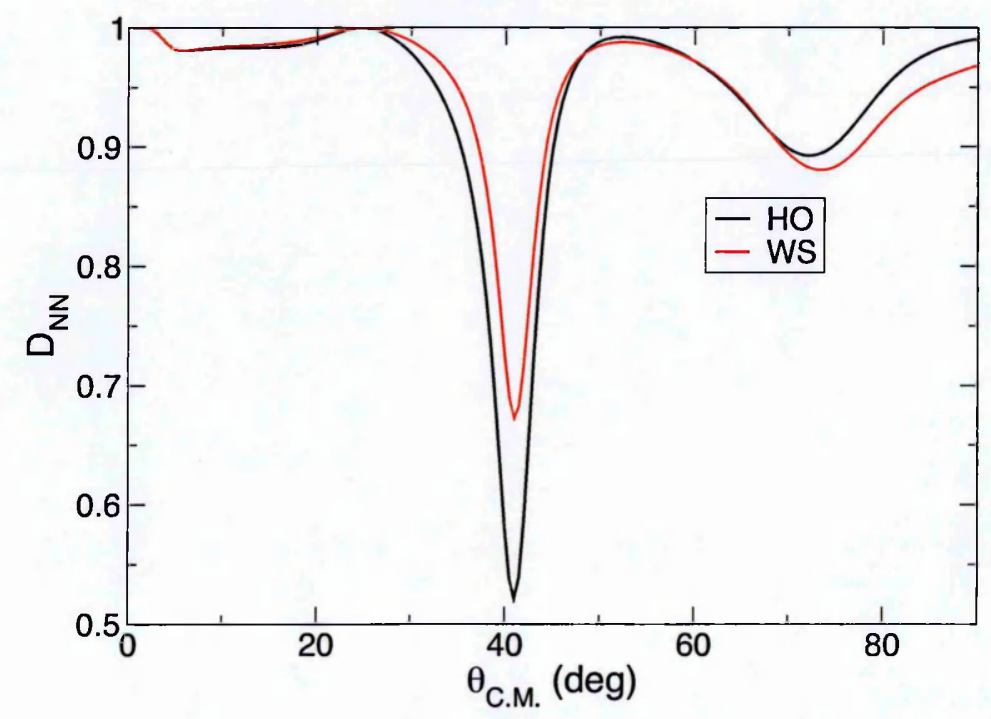

Figure 6.5: Calculation of $D_{N N}$ from spherical spin-spin interaction, without spin-orbit distorting potential, for elastic proton scattering from ${ }^{10} \mathrm{~B}$. 


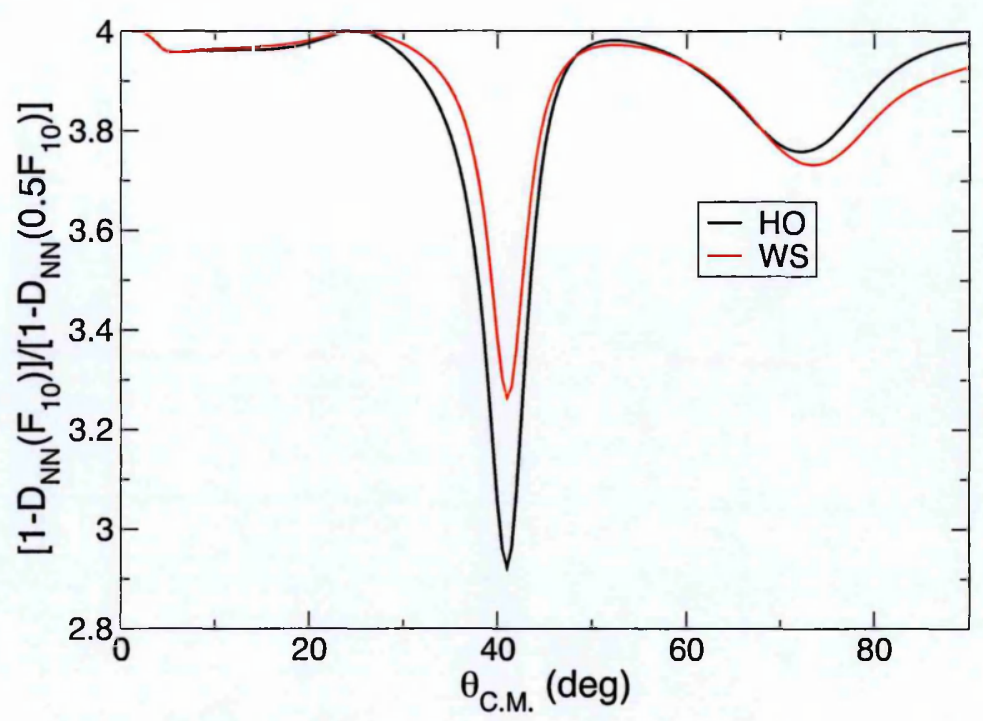

Figure 6.6: Ratio of the deviation of $1-D_{N N}$ for the spin-spin interaction $F_{10}(R)$ and $0.5 F_{10}(R)$, without spin-orbit distorting potential, for elastic proton scattering from ${ }^{10} \mathrm{~B}$.

\section{With Spin-Orbit}

Inclusion of the spin-orbit potential in calculating the distorted waves modifies the total nucleon-nucleus elastic scattering amplitude to Eq. 2.40. Using this scattering amplitude to calculate the ratio-to-Rutherford cross section, with the spin-spin amplitude limited to the spherical $f_{10}\left(\mu_{f}, M_{f} ; \mu_{i}, M_{i} ; \theta\right)$ term, gives the red curves in Figure 6.7. The spin-orbit potential has the effect of filling in the minimum at $\sim 40^{\circ}$ in Figure $6.4(\mathrm{~b})$ and increasing the difference between the spin-spin and non spin-spin amplitudes. This diminishes the effect of the spin-spin interactions on the observables which is why they have negligible effect on the cross section in Figure $6.7^{1}$ and why the deviation of $D_{N N}$ from unity, shown in Figure 6.8, is greatly reduced compared to Fig. 6.5.

The cross section in Figure 6.7 is much closer to the experimental measurements given in Fig. 3 of [35]. It is exactly the same as their optical model calculation (dashed curve in Fig. 3 [35]) because the same optical model parameters, for the $\boldsymbol{I}$-independent interactions, are used in this work, see Section 2.3.

To see if the inclusion of a spin-orbit term in the distorting potential changes how the deviation of $D_{N N}$ from unity is related to the strength of the spin-spin interaction, the ratio of $1-D_{N N}$ for $F_{10}(R)$ and $0.5 \times F_{10}(R)$ is plotted in Figure 6.9. In agreement with Fig. 6.6, when the strength of the spin-spin potential changes by a factor of 2 the deviation of $D_{N N}$ from 1 changes by a factor of 4 . Again, the dependence of $1-D_{N N}$ on the strength of $F_{10}(R)$ is to second order.

\footnotetext{
${ }^{1}$ The effect of all the spin-spin interactions discussed in this work on the calculation of the cross section is negligible so it will not be plotted again.
} 


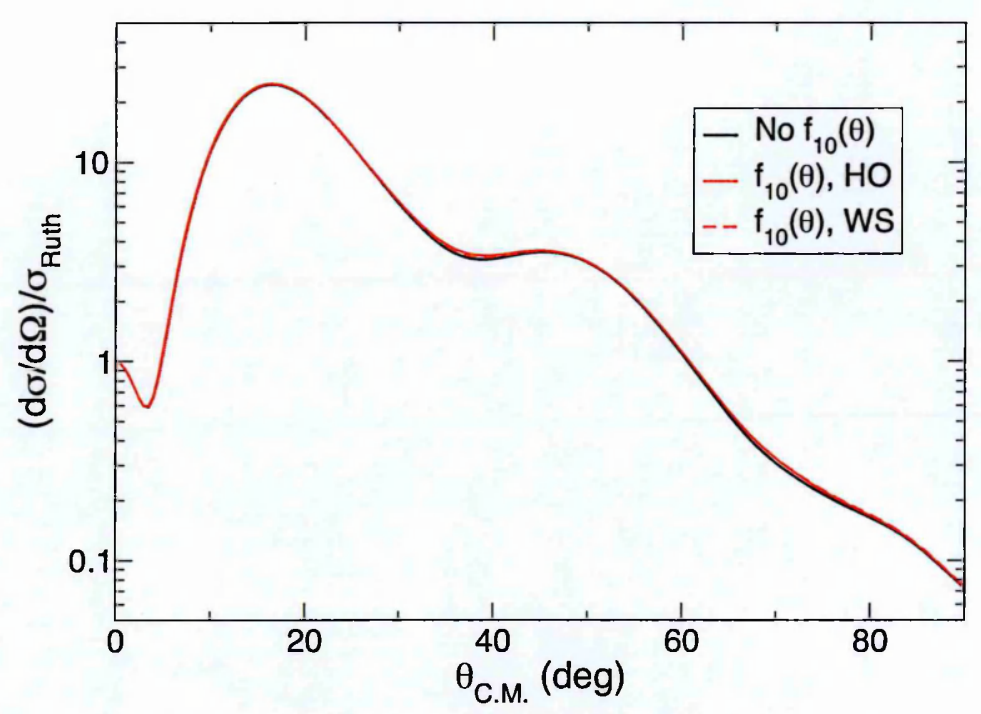

Figure 6.7: Ratio-to-Rutherford cross section excluding (black curve) and including (red curves) the spherical spin-spin amplitude for elastic proton scattering from ${ }^{10} \mathrm{~B}$. The total scattering amplitude was calculated with a spin-orbit distorting potential and the spinspin interaction was calculated with both HO (solid red line) and WS (dashed red line) single-particle wavefunctions.

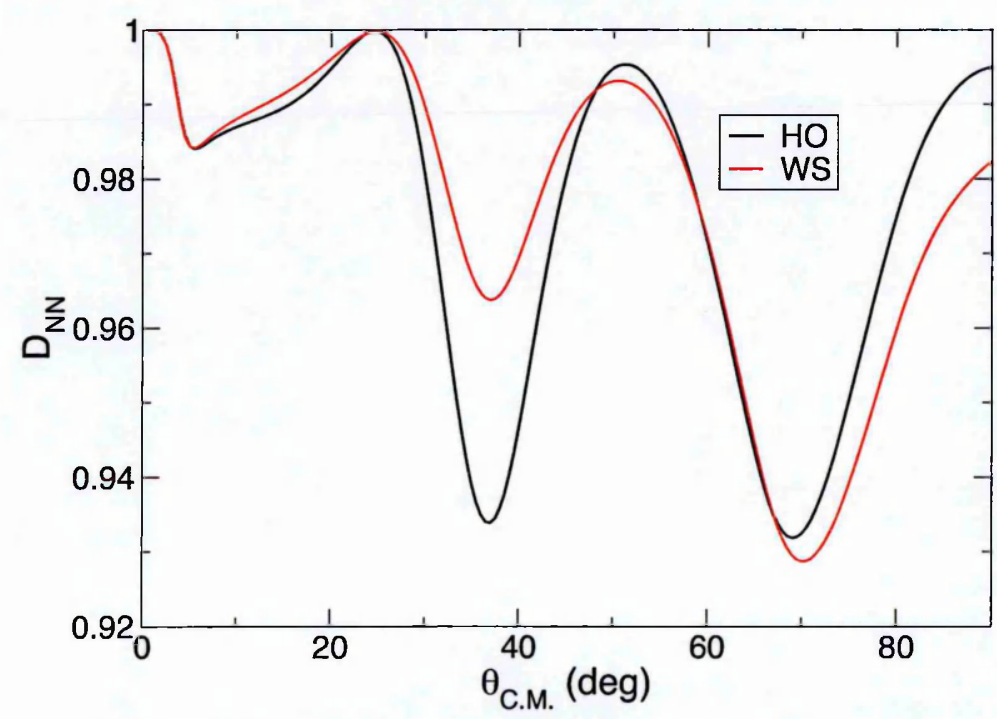

Figure 6.8: Calculation of $D_{N N}$ from spherical spin-spin interaction, with spin-orbit distorting potential, for elastic proton scattering from ${ }^{10} \mathrm{~B}$. 


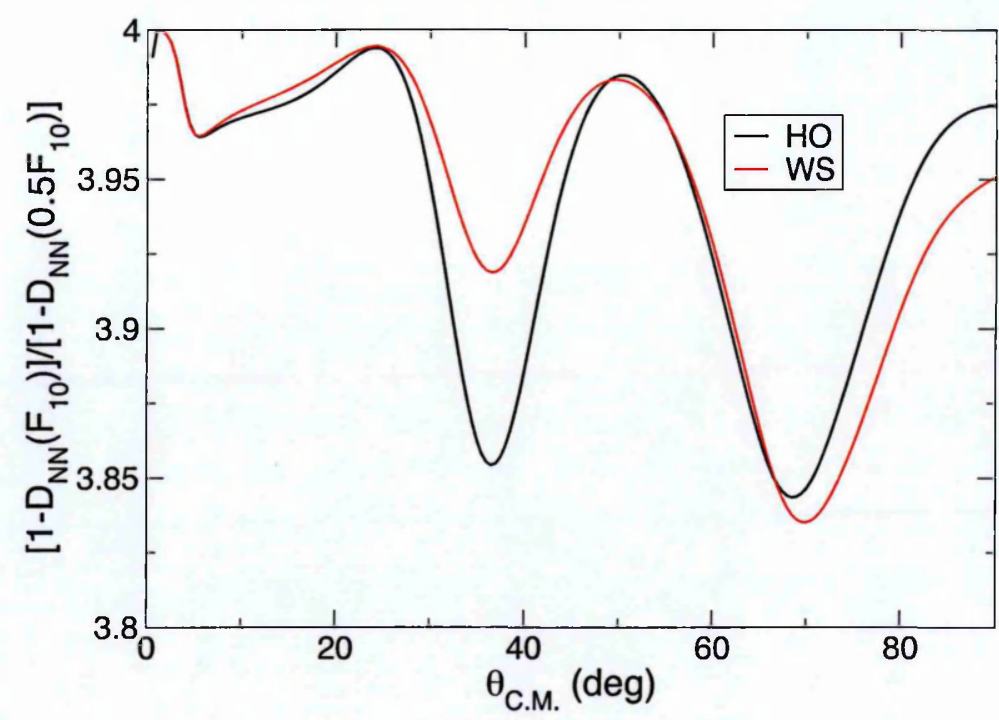

Figure 6.9: Ratio of the deviation of $D_{N N}$ from unity for the spherical spin-spin interaction $F_{10}(R)$ and $0.5 F_{10}(R)$, with spin-orbit distorting potential, for elastic proton scattering from ${ }^{10} \mathrm{~B}$.

\subsubsection{All Spin-Spin Interactions (Direct terms)}

Figure 6.10 shows the calculation of $D_{N N}$ for all the spin-spin interactions obtained from the folding of the direct terms in the NN interaction. The spin-spin interactions used in this calculation of $D_{N N}$ therefore include contributions from both the $v_{N N}^{\sigma \sigma}(r) \boldsymbol{\sigma}_{a} \cdot \boldsymbol{\sigma}_{b}$ and $v_{N N}^{t r}(r) \boldsymbol{S}_{a b}$ folded potentials, i.e. $U_{k_{I} k}(\boldsymbol{R})=U_{k_{I} k}^{\sigma \sigma}(\boldsymbol{R})+U_{k_{I} k}^{t r}(\boldsymbol{R})$, except for $U_{34}(\boldsymbol{R})$ which only has contributions from $U_{34}^{t r}(\boldsymbol{R})$ because angular momentum coupling requires that, $k \leq 2$, for $U_{k_{I} k}^{\sigma \sigma}(\boldsymbol{R})$.

In both Figs. 6.10(a) and 6.10(b), for angles below $45^{\circ}$, the contribution of the $U_{10}(R)$ spin-spin interaction to $D_{N N}$ is significant, but so is the contribution from McAbee's $U_{32}(\boldsymbol{R})$ potential that had been neglected in early calculations [20,21]. Above $45^{\circ}$, the only significant contribution comes from $U_{10}(R)$.

The depths of the two main troughs in the $D_{N N}$ curve at around $35^{\circ}$ and $70^{\circ}$ are significantly different for the two different types of single-particle wavefunction used to calculate the spin-spin interactions. For the HO wavefunction, the first trough at $35^{\circ}$ is deeper than the second. The WS wavefunction gives the opposite result; the trough at $70^{\circ}$ is deeper than the first. The sensitivity of $D_{N N}$ to the choice of single-particle wavefunction will be examined further in Section 6.6. 


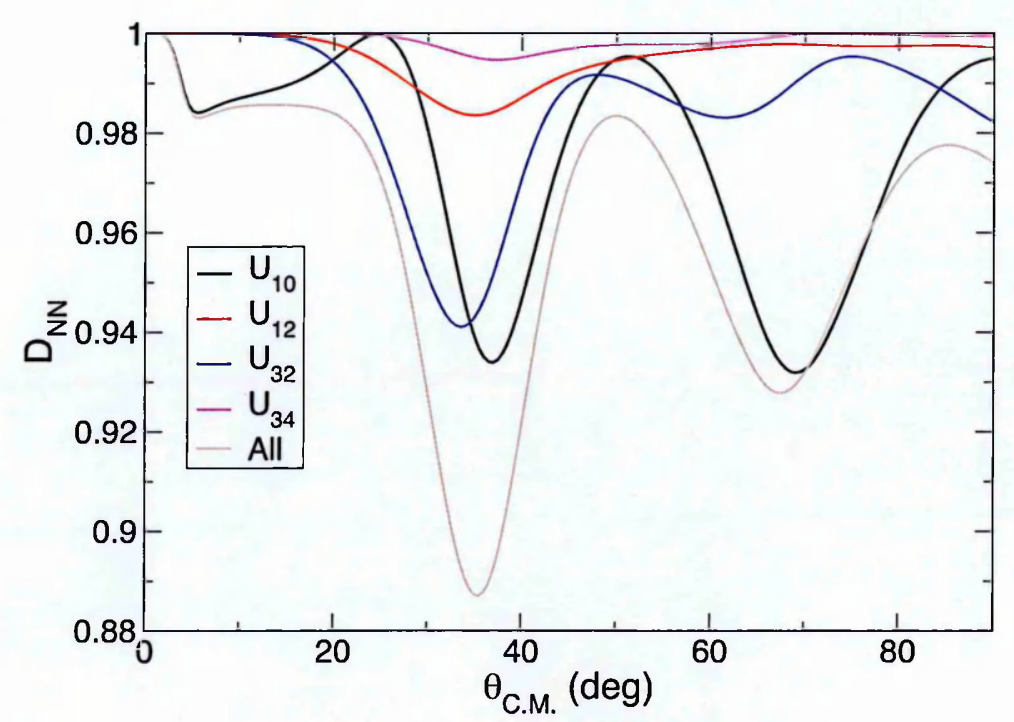

(a) HO single-particle wavefunction

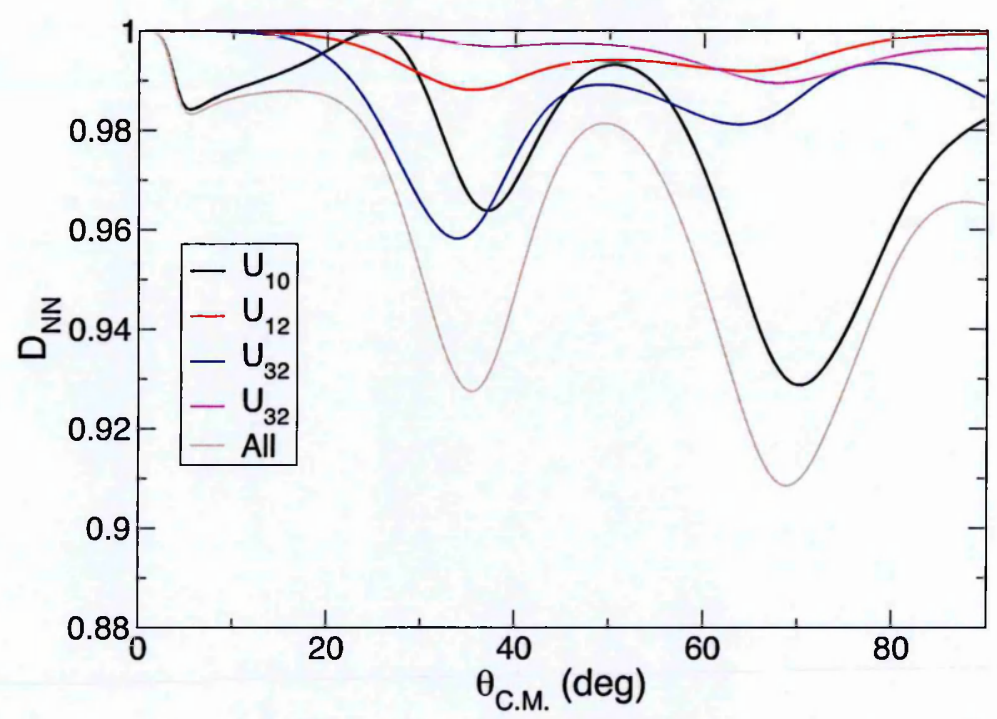

(b) WS single-particle wavefunction

Figure 6.10: $D_{N N}$ for elastic proton scattering from ${ }^{10} \mathrm{~B}$, calculated using all spin-spin interactions from folding of direct terms in the NN interaction. Spin-spin interactions calculated using (a) HO and (b) WS single-particle wavefunctions.

\subsection{Central Exchange Spin-Spin Interaction}

Section 5.2 describes the folding of the central single-nucleon knock-on exchange (SNKE) term, $\hat{v}_{p p}^{\sigma \sigma}\left(r_{p}\right) \boldsymbol{\sigma}_{0} \cdot \boldsymbol{\sigma}_{1} P_{01}^{r}+\hat{v}_{p n}^{\sigma \sigma}\left(r_{n}\right) \boldsymbol{\sigma}_{0} \cdot \boldsymbol{\sigma}_{2} P_{02}^{r}$, using the zero-range pseudo-potential from Eq. 4.20. This folding gives spin-spin interactions of the form $U_{k_{I} k}(\boldsymbol{R})=F_{k_{I} k}(R) \boldsymbol{S}_{k_{I} k}$. The form factors from this folding, $\hat{F}_{k_{k} k}^{\sigma \sigma}(R)$, are given in Eqs. 5.3 to 5.5 for protons elastically scattered from ${ }^{10} \mathrm{~B}$ and are simply proportional to $u_{1 \frac{3}{2}}(r)^{2}$.

For the following discussion, the coefficient, $\hat{J}_{\sigma \sigma}(Q)$, is evaluated for the simple case 
of $Q=k_{c m}$. This gives $\hat{J}_{\sigma \sigma}^{p p}(Q)=-134.775+77.363 \imath \mathrm{MeV} \cdot \mathrm{fm}^{3}$ for like particles and $\hat{J}_{\sigma \sigma}^{p n}(Q)=-75.395-5.501 \imath \mathrm{MeV} \cdot \mathrm{fm}^{3}$ for unlike particles. The effect of a $\theta$-dependent $Q$ on $\hat{J}_{\sigma \sigma}(Q)$ is discussed in Section 6.5. The form factors, $\hat{F}_{k_{I} k}^{\sigma \sigma}(R)$, from Eqs. 5.3 to 5.5 are plotted in Figure 6.11. These form factors can be checked using their volume integrals in the same way as those derived from the direct terms in the NN interaction. Using the checks detailed in Section 5.5.1 the numbers obtained from the volume integrals are given in Table 6.3.

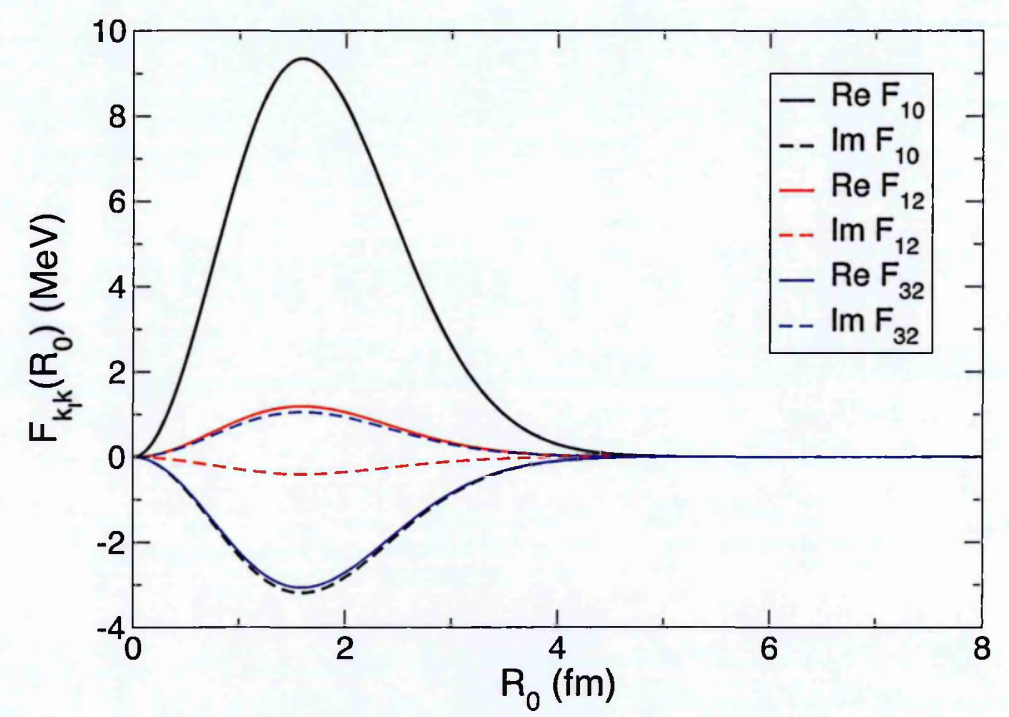

(a) Harmonic oscillator wavefunction

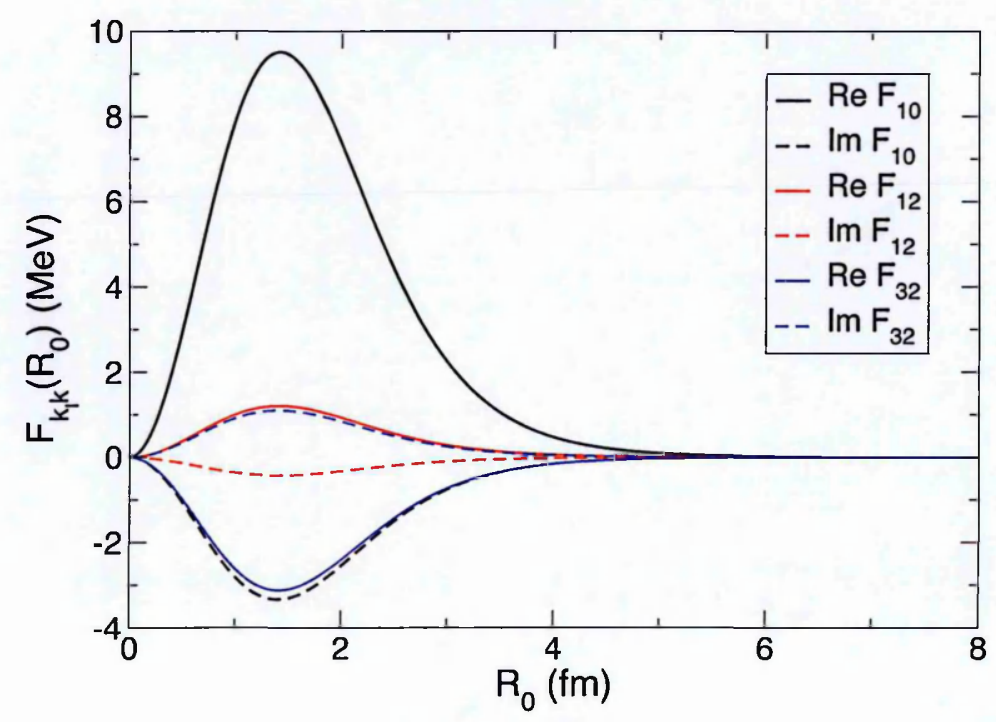

(b) Woods-Saxon wavefunction

Figure 6.11: Spin-spin interactions calculated from the folding of the exchange central $\hat{v}_{p p}^{\sigma \sigma}\left(r_{p}\right) \boldsymbol{\sigma}_{0} \cdot \boldsymbol{\sigma}_{1} P_{01}^{r}+\hat{v}_{p n}^{\sigma \sigma}\left(r_{n}\right) \boldsymbol{\sigma}_{0} \cdot \boldsymbol{\sigma}_{2} P_{02}^{r}$ term in the NN interaction, using (a) harmonic oscillator wavefunction and (b) Woods-Saxon wavefunction, for elastic proton scattering from ${ }^{10} \mathrm{~B}$. 


\begin{tabular}{|c||c|c|c|}
\hline$k_{I}, k$ & Eq. no. & Wfn & Result \\
\hline \hline 1,0 & \multirow{2}{*}{5.58} & HO & $\begin{array}{c}860.272-294.162 \imath \mathrm{MeV} . \mathrm{fm}^{3} \\
860.272-294.162 \imath \mathrm{MeV} . \mathrm{fm}^{3}\end{array}$ \\
\hline 1,2 & 5.60 & HO & $\begin{array}{c}55.057-18.826 \imath \mathrm{MeV} . \mathrm{fm}^{5} \\
67.019-22.322 \imath \mathrm{MeV} . \mathrm{fm}^{5}\end{array}$ \\
\hline \multirow{2}{*}{3,2} & 5.60 & HO & $\begin{array}{c}-143.042+48.912 \imath \mathrm{MeV} . \mathrm{fm}^{5} \\
-174.121+57.993 \imath \mathrm{MeV} . \mathrm{fm}^{5}\end{array}$ \\
\hline
\end{tabular}

Table 6.3: The volume integrals, as defined in Section 5.5.1, of the spin-spin interactions calculated from the folding of the central exchange term in the NN interaction.

Comparing the central exchange form factors, $\hat{F}_{k_{I}}^{\sigma \sigma}(R)$, in Fig. 6.11 with the central direct form factors, $F_{k_{I} k}^{\sigma \sigma}(R)$, in Fig. 6.2 reveals that the magnitudes of the real parts are larger for the exchange form factors and of opposite sign. The imaginary parts of $\hat{F}_{k_{I} k}^{\sigma \sigma}(R)$ also have opposite signs but have similar magnitudes compared to $F_{k_{I} k}^{\sigma \sigma}(R)$, except for the spherical terms where $F_{10}^{\sigma \sigma}(R) \sim 1.5 \times \hat{F}_{10}^{\sigma \sigma}(R)$ at their peaks.

The opposite signs of the spin-spin potentials from the folding of the direct $v_{N N}^{\sigma \sigma}(r) \sigma_{a} \cdot \sigma_{b}$ and exchange $\hat{v}_{N N}^{\sigma \sigma}(r) \sigma_{a} \cdot \sigma_{b} P_{a b}^{r}$ terms in the NN interaction leads to considerable cancellation. The cause of this can be found by examining how SNKE is formally included in the folding model using Eq. 4.15 and 4.16. The direct interaction $v_{N N}^{\sigma \sigma}(r)$ equals the exchange potential $\hat{v}_{N N}^{\sigma \sigma}(r)$ except that in the latter the sign of the odd-state terms is changed, this leads to significant cancellation between the central direct and exchange terms especially for the odd-state terms. If the potentials for the direct and exchange odd state terms were replaced with a zero range delta function they would cancel completely [44].

As with the direct terms, there is not a significant difference between the central exchange spin-spin interactions calculated from the HO (Figure 6.11(a)) and the WS (Figure 6.11(b)) wavefunctions. Those calculated using the latter are slightly stronger but the use of different wavefunctions has little effect on the shape of the potentials.

Figure 6.12 shows the calculation of $D_{N N}$ for all the spin-spin interactions obtained from the folding of central exchange term in the NN interaction. In both Figs. 6.12(a) and $6.12(\mathrm{~b})$, for angles below $45^{\circ}$, the contribution of the $U_{10}(R)$ and $U_{32}(\boldsymbol{R})$ spin-spin interactions to the deviation of $D_{N N}$ from unity are the most significant. Above $45^{\circ}$ the most significant contribution comes from $U_{10}(R)$ alone. This is consistent with the $D_{N N}$ calculated using the spin-spin interactions obtained from the folding of the direct terms shown in Figure 6.10.

The largest deviations of $D_{N N}$ from unity in Figure 6.12 are at the same angles as Figure 6.10, around $35^{\circ}$ and $70^{\circ}$, irrespective of which single-particle wavefunction used. For the HO wavefunction the first trough at $35^{\circ}$ is much deeper than the second. However, for the WS wavefunction the two troughs have a similar depth. This sensitivity of $D_{N N}$ 


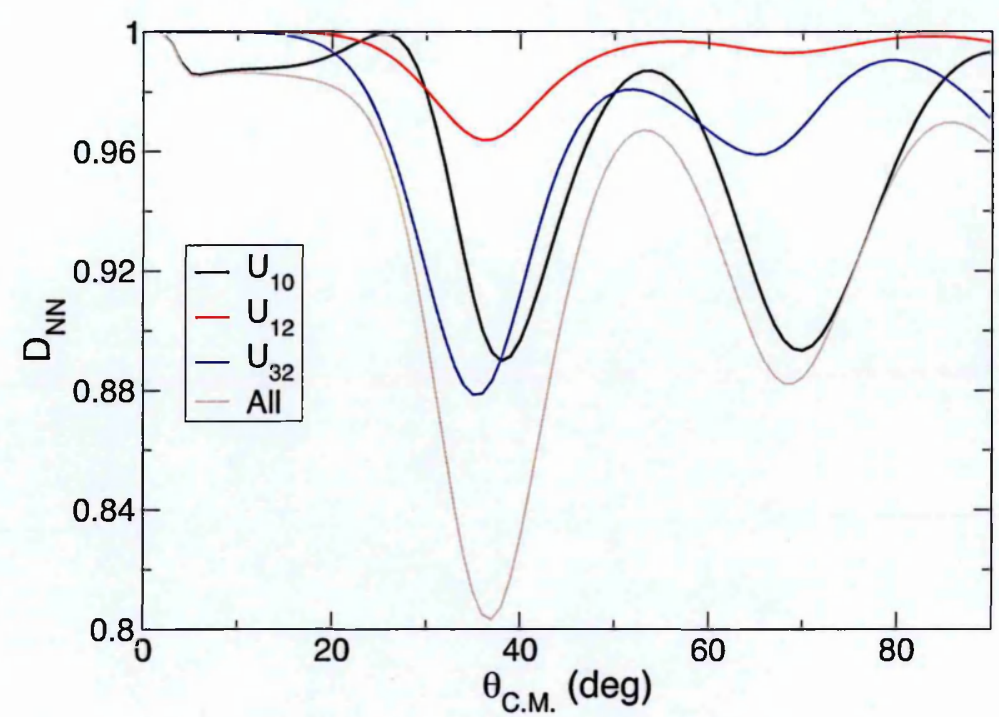

(a) HO single-particle wavefunction

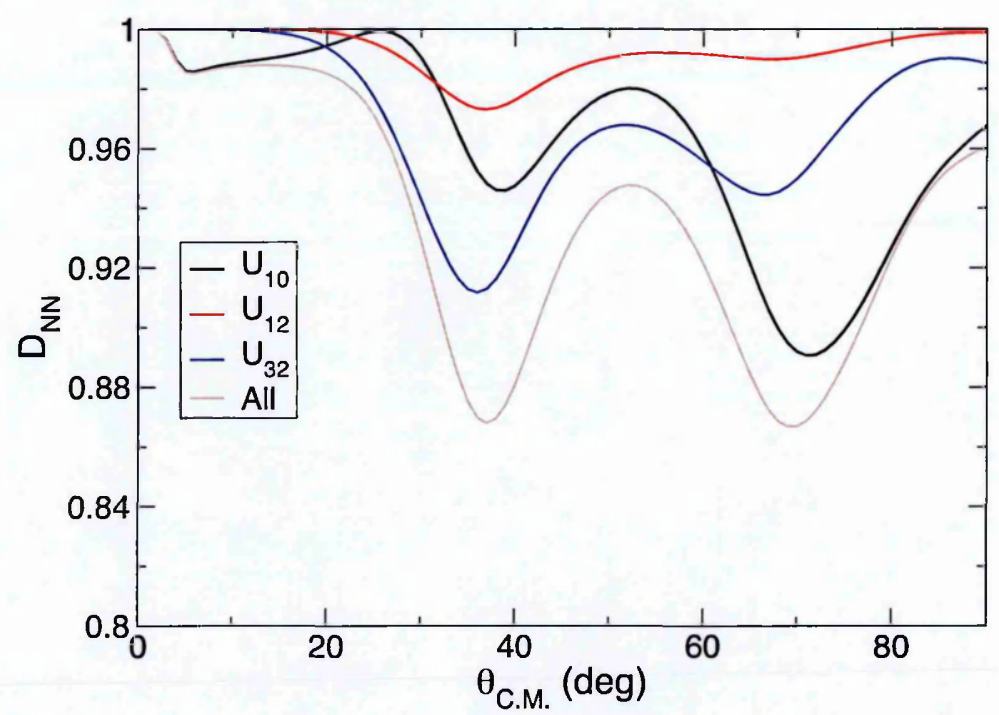

(b) WS single-particle wavefunction

Figure 6.12: $D_{N N}$ for elastic proton scattering from ${ }^{10} \mathrm{~B}$, calculated using all spin-spin interactions from folding of the central exchange term in the NN interaction. Spin-spin interactions calculated using (a) HO and (b) WS single-particle wavefunctions.

to different single-particle wavefunctions will be discussed in Section 6.6.

\subsection{Tensor Exchange Spin-Spin Interaction}

The folding of the tensor SNKE term, $\hat{v}_{p p}^{t r}\left(r_{p}\right) \boldsymbol{S}_{01} P_{01}^{r}+\hat{v}_{p n}^{t r}\left(r_{n}\right) \boldsymbol{S}_{02} P_{02}^{r}$, using the zerorange pseudo-potential from Eq. 4.27 is detailed in Section 5.3. Each of the spin-spin interactions derived in Section 5.3 depends on the coefficient $\hat{J}_{t r}(Q)$. For the following 
discussion, $\hat{J}_{t r}(Q)$ will be evaluated for the simple case of $Q=k_{c m}$. The effect of a $\theta$-dependent $Q$ on $\hat{J}_{t r}(Q)$ will be examined in Section 6.5. For $Q=k_{c m}, \hat{J}_{t r}^{p p}(Q)=$ $3.541-2.267 \imath \mathrm{MeV} \cdot \mathrm{fm}^{5}$ for like particles and $\hat{J}_{t r}^{p n}(Q)=3.373-1.563 \imath \mathrm{MeV} \cdot \mathrm{fm}^{5}$ for unlike particles.

The form of the zero-range pseudo-potential used to approximate the tensor SNKE term resulted in the splitting of the folded potential into three terms Term A, detailed in Section 5.3.1, Term B, detailed in Section 5.3.2 and Term C, detailed in Section 5.3.3. The spin-spin interactions from Term A will be examined first.

\section{NN Exchange Tensor Term A}

The folding of this term is the only one of the three tensor exchange terms that gives spin-spin interactions of the form $U_{k_{I} k}(\boldsymbol{R})=F_{k_{I} k}(R) \boldsymbol{S}_{k_{I} k}$. The form factors from this folding, $\hat{F}_{k_{I} k}^{t r A}(R)$, are given in Eqs. 5.21 to 5.25 and are plotted in Figure 6.13 for protons elastically scattered from ${ }^{10} \mathrm{~B}$.

Figure 6.13 shows that some of these spin-spin potentials have more radial structure than those from the folding of the central exchange term in Figure 6.11. This is because the $\hat{F}_{k_{I} k}^{t r A}(R)$ form factors depend on the superposition of second order differential operators acting on the single-particle wavefunction. They are also much weaker than the folded potentials from the direct tensor term in the NN interaction shown in Figure 6.3.

It can also be seen in Figure 6.13 that the spherical $\hat{F}_{10}^{\operatorname{tr} A}(R)$ form factor is again the strongest. The only form factor with an even rank in $\boldsymbol{I}, \hat{F}_{22}^{t r A}(R)$, is also relatively strong when compared to the other form factors, and has an opposite sign. The WS wavefunction has the effect of making the magnitudes of the form factors slightly stronger compared to those calculated using the $\mathrm{HO}$ wavefunction.

\section{NN Exchange Tensor Term B}

The folding of this term gives spin-spin interactions of the form $\hat{F}_{\dot{k}_{I} k}^{\operatorname{tr} B}(R)\left[Y_{k}(\hat{\boldsymbol{R}}) \times \tau_{k_{I}}(\boldsymbol{I})\right]$. $\left[\tau_{1}\left(\boldsymbol{\sigma}_{0}\right) \times \mathscr{Y}_{2}\left(-\imath \nabla_{\boldsymbol{R}}\right)\right]$, where the solid spherical harmonic acts on the initial distorted wavefunction. The form factors $\hat{F}_{k_{I} k}^{t r B}(R)$ are given in Eqs. 5.30 to 5.32 and are shown in Figure 6.14 for protons elastically scattered from ${ }^{10} \mathrm{~B}$. These spin-spin potentials have a different structure than those discussed previously, which have the form $U_{k_{I} k}(\boldsymbol{R})=$ $F_{k_{I} k}(R) \boldsymbol{S}_{k_{I} k}$, so it is difficult to compare them directly.

Like the central exchange form factors, $\hat{F}_{k_{I} k}^{t r B}(R)$ is simply proportional to $u_{1 \frac{3}{2}}(R)^{2}$. This is because the differential operator acts solely on the distorted wave and does not affect the valence nucleon wavefunction. The spherical $\hat{F}_{10}^{\operatorname{tr} B}(R)$ potential is the strongest and the $\hat{F}_{32}^{\operatorname{tr} B}(R)$ interaction is stronger than the tensor $\hat{F}_{12}^{\operatorname{tr} B}(R)$ potential. The signs of the 
spin-spin interactions are determined in Eqs. 5.30 to 5.32 and there is very little difference between the spin-spin interactions calculated using the two different radial wavefunctions.

\section{NN Exchange Tensor Term C}

The folding of this term gives spin-spin interactions on the form $\hat{F}_{k_{I} k E}^{t r C}(R)\left[Y_{k}(\hat{\boldsymbol{R}}) \times\right.$ $\left.\tau_{1}\left(\boldsymbol{\sigma}_{0}\right)\right]_{E \epsilon}\left[\tau_{k_{I}}(\boldsymbol{I}) \times \nabla\right]_{E-\epsilon}$ where the grad acts on the initial distorted wave. The numerous form factors are given in Eqs. 5.38 to 5.48 and are shown in Figure 6.15. As there are so many form factors they have been split between two different plots for interactions calculated using HO wavefunction Figure 6.15(a) and WS wavefunction Figure 6.15(b). Like Term B, these spin-spin potentials have a different structure to those discussed previously, so it is difficult to compare them directly.

The form factors in Figure 6.15(a) have a varied radial structure because they depend on the superposition of first order differential operators acting on the single-particle wavefunction. The spin-spin potentials calculated using the different single-particle wavefunctions don't differ in structure but the potentials are slightly stronger when calculated using the WS potential.

\subsubsection{Total NN Exchange Tensor Interaction}

Figure 6.16 shows the calculation of $D_{N N}$ for all three terms obtained from the folding of the tensor exchange term in the NN interaction. The deviation of $D_{N N}$ from unity caused by tensor exchange Term A is very small. The largest value of $1-D_{N N}$ for this term is 0.003. Term B causes the most significant deviation of $D_{N N}$ from unity and causes the large trough in the $D_{N N}$ curve for all three tensor exchange terms just below $40^{\circ}$. Tensor exchange Term C also causes significant deviation of $D_{N N}$ from unity just below $40^{\circ}$.

When the spin-spin interactions from the folding of tensor exchange Term $\mathrm{C}$ are calculated with a WS single-particle wavefunction $1-D_{N N}$ is also significant at larger angles. At $66^{\circ}, 1-D_{N N}=0.974$ for this term. However, cancellation between the terms means that the $D_{N N}$ curve for all three tensor exchange terms deviates from unity by a similar amount, at large angles, when calculated using the WS wavefunction, compared to the calculation using the $\mathrm{HO}$ wavefunction. The total $D_{N N}$ curve for the $\mathrm{HO}$ wavefunction leads to a more significant deviation of $D_{N N}$ from unity at the trough just below $40^{\circ}$.

Section 5.5.2 details the alternative calculation of the spin-spin interactions derived from the folding of the tensor exchange term in the NN interaction. This check results in four separate terms (see Sections 5.5.3 to 5.5.6) different from terms A, B and C discussed here. Using all the terms in either method to calculate $D_{N N}$ for elastic proton scattering from ${ }^{10} \mathrm{~B}$ leads to the same curve, shown in brown in Figure 6.16. 


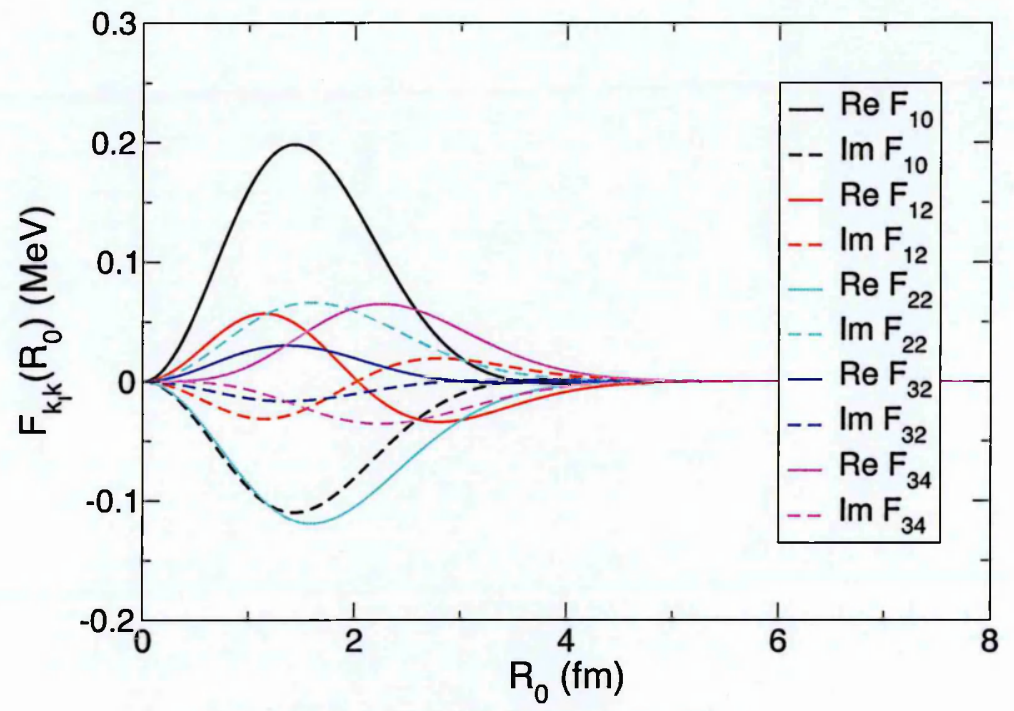

(a) Harmonic oscillator wavefunction

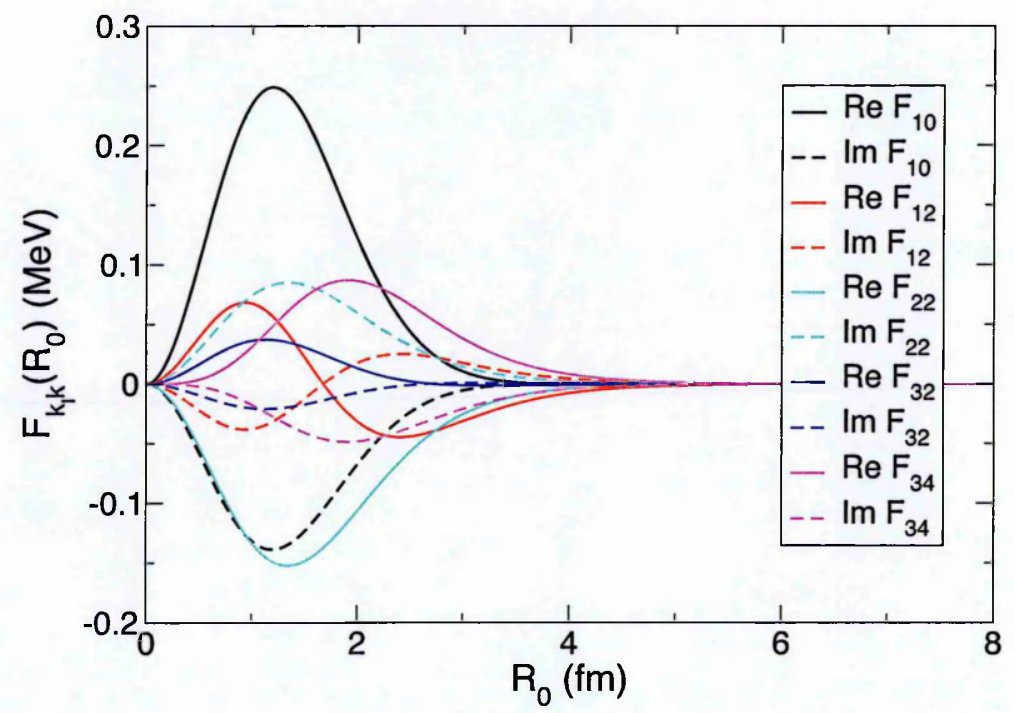

(b) Woods-Saxon wavefunction

Figure 6.13: Spin-spin interactions calculated from the folding of tensor exchange Term A, using (a) harmonic oscillator wavefunction and (b) Woods-Saxon wavefunction, for elastic proton scattering from ${ }^{10} \mathrm{~B}$. 


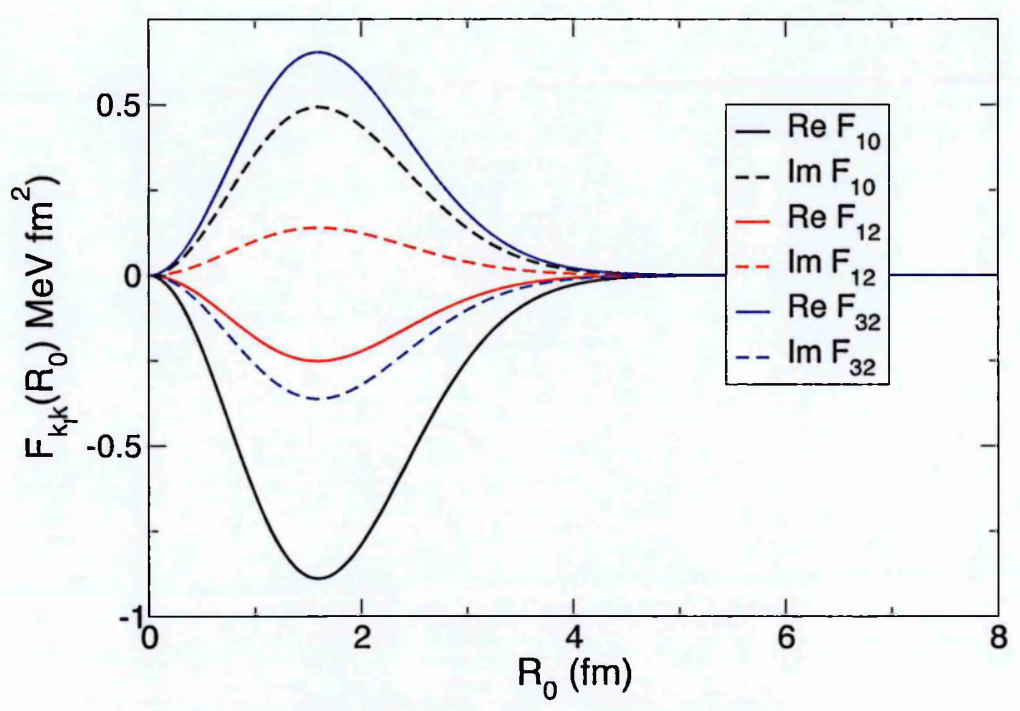

(a) Harmonic oscillator wavefunction

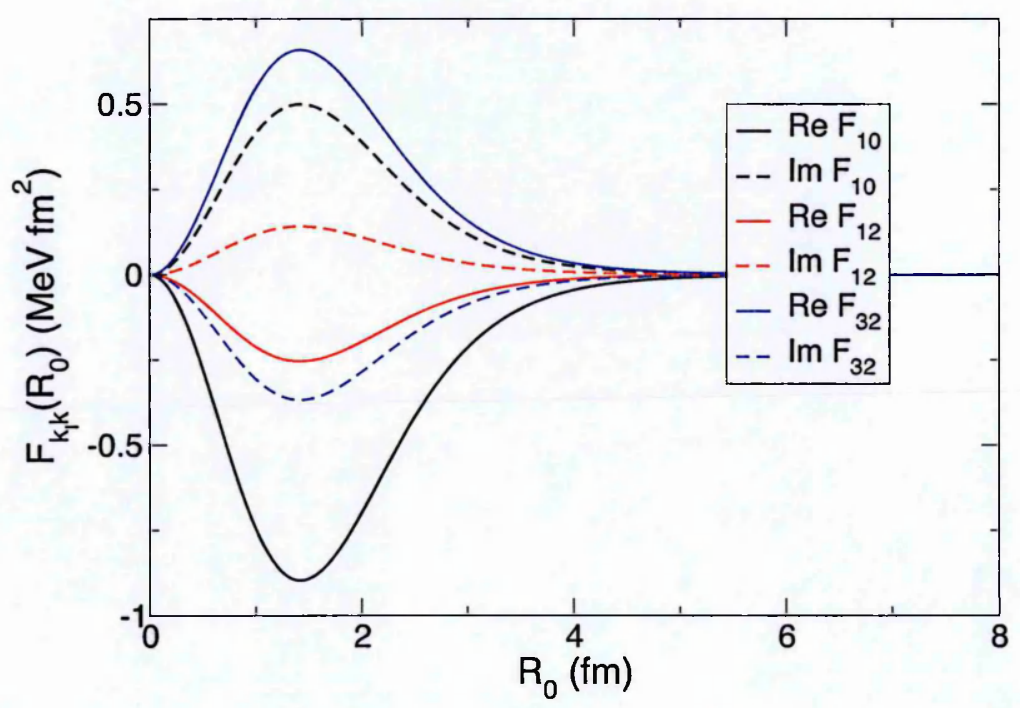

(b) Woods-Saxon wavefunction

Figure 6.14: Spin-spin interactions calculated from the folding of tensor exchange Term $\mathrm{B}$, using (a) harmonic oscillator wavefunction and (b) Woods-Saxon wavefunction, for elastic proton scattering from ${ }^{10} \mathrm{~B}$. 


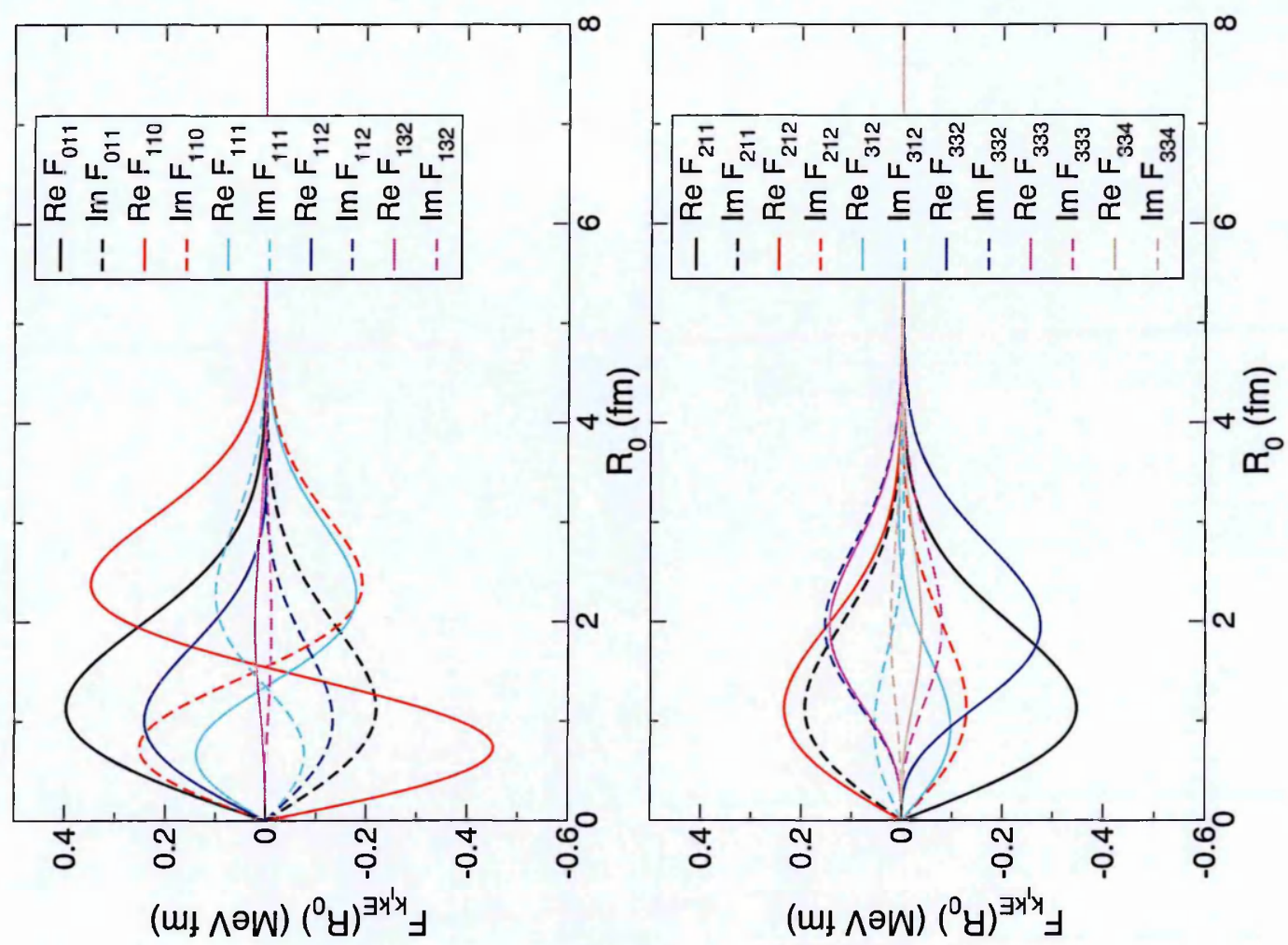

(a) Harmonic oscillator wavefunction

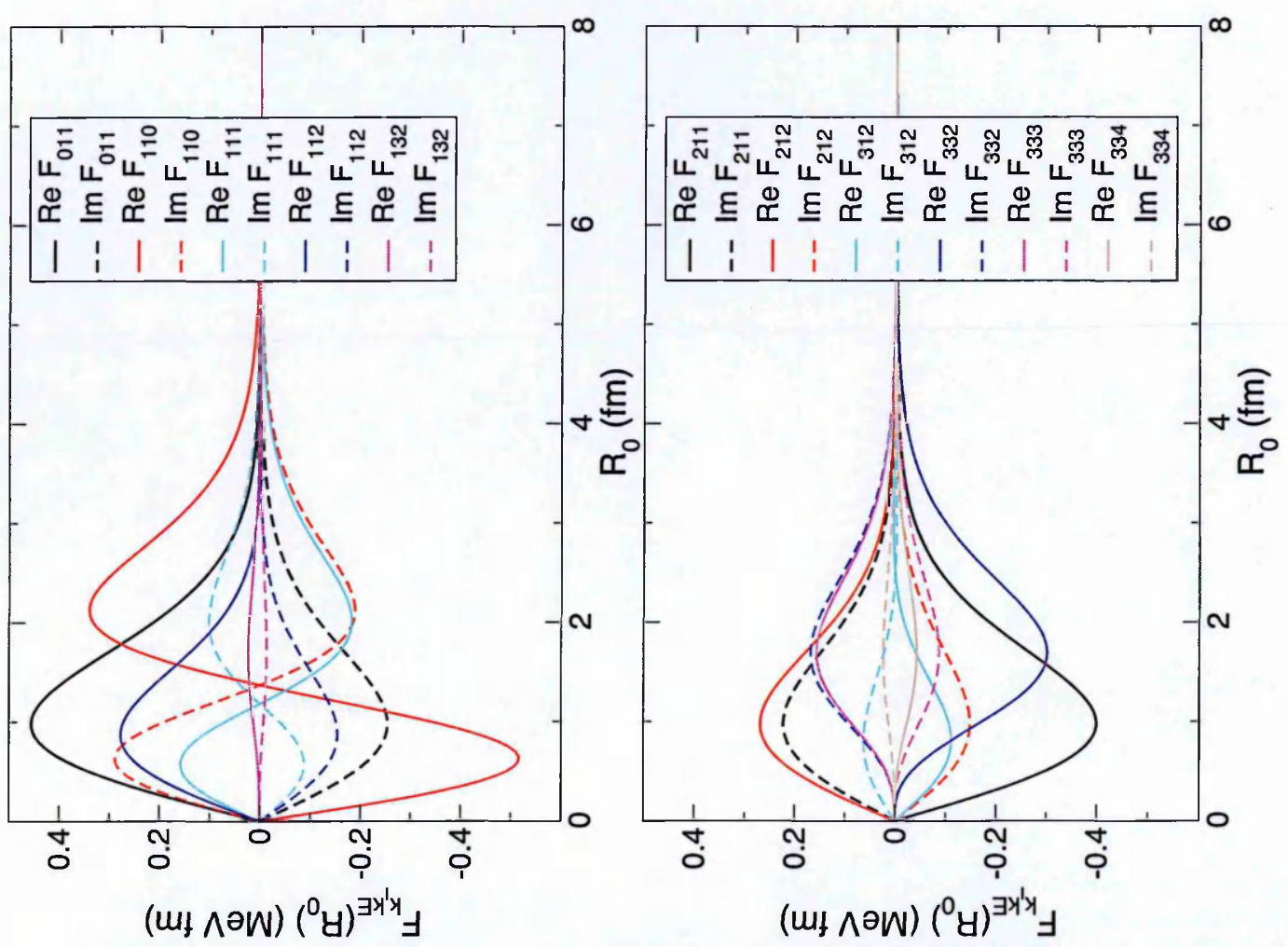

(b) Woods-Saxon wavefunction

Figure 6.15: Spin-spin interactions calculated from the folding of tensor exchange Term $\mathrm{C}$, using (a) harmonic oscillator wavefunction and (b) Woods-Saxon wavefunction, for elastic proton scattering from ${ }^{10} \mathrm{~B}$. 


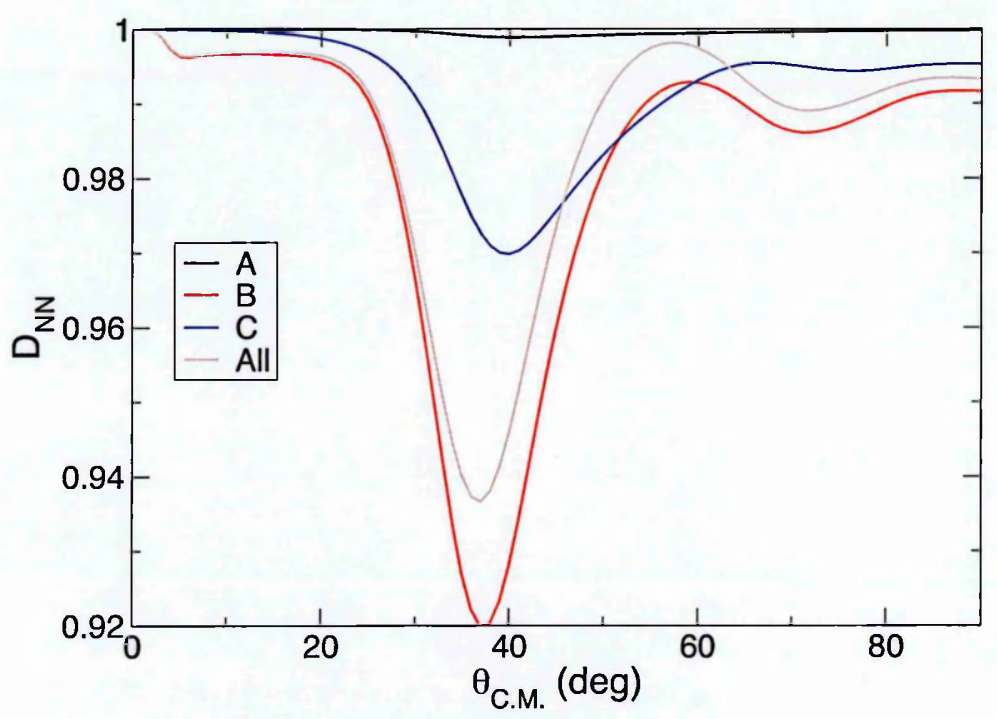

(a) HO single-particle wavefunction

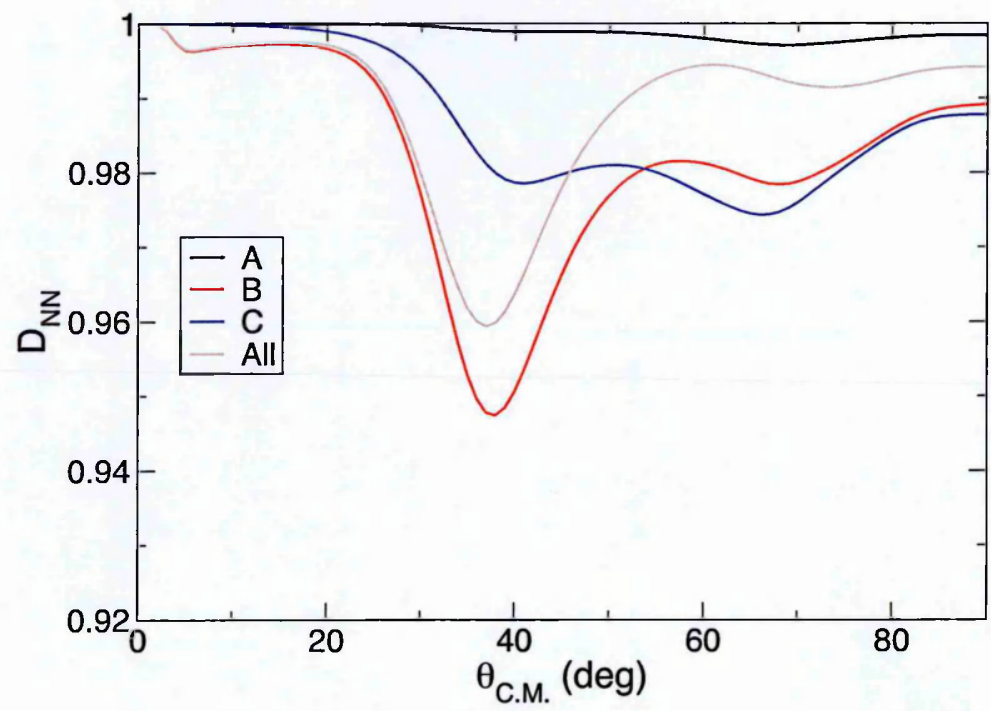

(b) WS single-particle wavefunction

Figure 6.16: $D_{N N}$ for elastic proton scattering from ${ }^{10} \mathrm{~B}$, calculated using all spin-spin interactions from folding of the tensor exchange term in the NN interaction. Spin-spin interactions calculated using (a) HO and (b) WS single-particle wavefunctions. 


\subsubsection{Effect of even $k_{I}$}

In Section 3.3.2, the model of ${ }^{10} \mathrm{~B}$ was changed from a valence proton and neutron in the $1 p_{\frac{3}{2}}$ shell to a valence proton and neutron hole. The only difference this makes to the folding formalism described in Chapters 3 and 5 occurs when the rank of the spin operator $\tau_{k_{I} q_{I}}(\boldsymbol{I})$ is even. When $k_{I}$ is greater than zero the reduced matrix element $\tau_{k_{I} q_{I}}^{M_{I} M_{i}}(\boldsymbol{I})$ must be multiplied by $(-)^{\left(k_{I}+1\right)}$ to change from a particle to a hole description [58].

The total spin-spin amplitude, $f\left(\mu_{f}, M_{f} ; \mu_{i}, M_{i} ; \theta\right)$, is given in Eq. 2.40

$$
f\left(\mu_{f}, M_{f} ; \mu_{i}, M_{i} ; \theta\right)=\sum_{k_{I} k} f_{k_{I} k}\left(\mu_{f}, M_{f} ; \mu_{i}, M_{i} ; \theta\right)
$$

All the individual spin-spin amplitudes, $f_{k_{I} k}\left(\mu_{f}, M_{f} ; \mu_{i}, M_{i} ; \theta, \phi\right)$, including those for the non-local tensor exchange terms, can be written as

$$
f_{k_{I} k}\left(\mu_{f}, M_{f} ; \mu_{i}, M_{i} ; \theta, \phi\right)=\sum_{q_{I}} \tau_{k_{I} q_{I}}^{M_{f} M_{i}}(\boldsymbol{I}) \hat{f}_{k_{I} q_{I}, k}\left(\mu_{f} ; \mu_{i} ; \theta, \phi\right)
$$

where $\hat{f}_{k_{I} q_{I}, k}\left(\mu_{f} ; \mu_{i} ; \theta, \phi\right)$ contains the partial wave sums, the integration over $R$ and the spherical harmonic $Y_{\ell^{\prime}, \mu_{i}-q_{I}-\mu_{f}}\left(\hat{\boldsymbol{k}}^{\prime}\right)$. Using the total spin-spin amplitude in Eq. 1.3 for $D_{N N}$ gives

$$
D_{N N}=\frac{\operatorname{Tr}\left[\sum_{k_{I} q_{I}, k, k_{I}^{\prime} q_{I}^{\prime}, k^{\prime}} \tau_{k_{I} q_{I}}^{M_{f} M_{i}}(\boldsymbol{I}) \hat{f}_{k_{I} q_{I}, k}\left(\mu_{f} ; \mu_{i} ; \theta, \phi\right) \sigma_{y} \tau_{k_{I}^{\prime} q_{I}^{\prime}}^{\dagger M_{f} M_{i}}(\boldsymbol{I}) \hat{f}_{k_{I}^{\prime} q_{I}^{\prime}, k^{\prime}}^{\dagger}\left(\mu_{f} ; \mu_{i} ; \theta, \phi\right) \sigma_{y}\right]}{\operatorname{Tr}\left[\sum_{k_{I} q_{I}, k, k_{I}^{\prime} q_{I}^{\prime}, k^{\prime}} \tau_{k_{I} q_{I}}^{M_{f} M_{i}}(\boldsymbol{I}) \hat{f}_{k_{I} q_{I}, k}\left(\mu_{f} ; \mu_{i} ; \theta, \phi\right) \tau_{k_{I}^{\prime} q_{I}^{\prime}}^{\dagger M_{f} M_{i}}(\boldsymbol{I}) \hat{f}_{k_{I}^{\prime} q_{I}^{\prime}, k^{\prime}}^{\dagger}\left(\mu_{f} ; \mu_{i} ; \theta, \phi\right)\right]}
$$

To make Eq. 6.6 describe holes and not particles the functions in the trace must be multiplied by $(-)^{1+k_{I}+1+k_{I}^{\prime}}$ (top and bottom) for $k_{I}>0$. This modification has no effect on the calculation of $D_{N N}$ because of the following property of the spin operators

$$
\operatorname{Tr}\left[\tau_{k q}(\boldsymbol{I}) \tau_{k^{\prime} q^{\prime}}^{\dagger}(\boldsymbol{I})\right]=(2 I+1) \delta_{k k^{\prime}} \delta_{q q^{\prime}}
$$

Taking the trace over the spin projection of the target gives

$$
D_{N N}=\frac{\operatorname{Tr}^{\prime}\left[\sum_{k_{I} q_{I}, k, k_{I}^{\prime} q_{I}^{\prime}, k^{\prime}} \delta_{k_{I} k_{I}^{\prime}} \delta_{q_{I} q_{I}^{\prime}} \hat{f}_{k_{I} q_{I}, k}\left(\mu_{f} ; \mu_{i} ; \theta, \phi\right) \sigma_{y} \hat{f}_{k_{I}^{\prime} q_{I}^{\prime}, k^{\prime}}^{\dagger}\left(\mu_{f} ; \mu_{i} ; \theta, \phi\right) \sigma_{y}\right]}{\operatorname{Tr}^{\prime}\left[\sum_{k_{I} q_{I}, k, k_{I}^{\prime} q_{I}^{\prime}, k^{\prime}} \delta_{k_{I} k_{I}^{\prime}} \delta_{q_{I} q_{I}^{\prime}} \hat{f}_{k_{I} q_{I}, k}\left(\mu_{f} ; \mu_{i} ; \theta, \phi\right) \hat{f}_{k_{I}^{\prime} q_{I}^{\prime}, k^{\prime}}^{\dagger}\left(\mu_{f} ; \mu_{i} ; \theta, \phi\right)\right]}
$$

where $\operatorname{Tr}^{\prime}$ is taken over the spin projections of the projectile alone. The phase change now becomes $(-)^{1+k_{I}+1+k_{I}^{\prime}} \rightarrow(-)^{1+k_{I}+1+k_{I}}=1$. So the phase change that results from 
transferring from the particle to hole model of ${ }^{10} \mathrm{~B}$ does not change the polarisation transfer coefficient $D_{N N}$ or the cross section.

More generally this result means that in the calculation of $D_{N N}$ only cross terms in the scattering amplitude with the same rank in the target spin survive the trace over the spin projection of the target. Cross terms with different $k_{I}$ will go to zero. This is another explanation why, in Appendix B, the cross term of the spin-zero amplitude $g(\theta)$, with $k_{I}=0$, and the spherical spin-spin amplitude $h_{10}(\theta)$, with $k_{I}=1$, is zero for the simple calculation of $D_{N N}$.

\subsection{All Spin-Spin Interactions and Q Dependence}

So far in this chapter the coefficients $\hat{J}_{\sigma \sigma}(Q)$ and $\hat{J}_{t r}(Q)$ (described in Section 4.5) from the zero-range pseudo potentials used to approximate the SKNE terms in the NN interaction have been calculated using the approximation $Q=k_{c m}$. As discussed in Section 4.5.3 this choice is reasonable for scattering in the forward direction. However the measurement of $D_{N N}$ for $200 \mathrm{MeV}$ protons elastically scattering from ${ }^{10} \mathrm{~B}$ detailed in [35] shows that the deviation of $D_{N N}$ from unity is most significant at large angles. Therefore in Section 4.5.3 the details of an approximation for the magnitude of $\boldsymbol{Q}$ was given that does better at large angles, $Q=k_{c m} \cos (\theta / 2)$.

Figure 6.17 shows the coefficients $\hat{J}_{\sigma \sigma}(Q)$ and $\hat{J}_{t r}(Q)$ for the two different approximations of $Q$. The introduction of a $\theta$-dependent $Q$ changes the values of $\hat{J}_{\sigma \sigma}(Q)$ and $\hat{J}_{t r}(Q)$ significantly as shown in Table 6.4 for $\theta=0$ and $\theta=90$.

\begin{tabular}{|c||c|c|c|}
\hline & & $\theta=0$ & $\theta=90$ \\
\hline \hline$\hat{J}_{\sigma \sigma}(Q)$ & pp & $-134.775+77.363 \imath$ & $-85.900+101.759 \imath$ \\
${\mathrm{MeV} . \mathrm{fm}^{3}}^{3}$ & pn & $-75.395-5.501 \imath$ & $-53.469-22.163 \imath$ \\
\hline$\hat{J}_{t r}(Q)$ & $\mathrm{pp}$ & $3.541-2.267 \imath$ & $9.011-0.643 \imath$ \\
$\mathrm{MeV} . \mathrm{fm}^{5}$ & $\mathrm{pn}$ & $3.373-1.563 \imath$ & $19.401-3.463 \imath$ \\
\hline
\end{tabular}

Table 6.4: Exchange coefficients $\hat{J}_{\sigma \sigma}(Q)$ and $\hat{J}_{t r}(Q)$ at $\theta=0$ and $\theta=90$ for like (pp) and unlike (pn) particles.

The calculation of the polarisation transfer coefficient $D_{N N}$ is plotted in Figure 6.18 for all the spin-spin interactions derived in this work. The black curve shows $D_{N N}$ evaluated using $Q=k_{c m}$ to calculate the spin-spin interactions from the folding of the central and tensor exchange terms in the NN interaction. The red curve in Figure 6.18 shows the same calculation but using $Q=k_{c m} \cos (\theta / 2)$.

The cancellation between direct and exchange terms means that the calculation of $D_{N N}$ plotted in the black curve of Figure 6.18 is not simply the combination of the $D_{N N}$ 


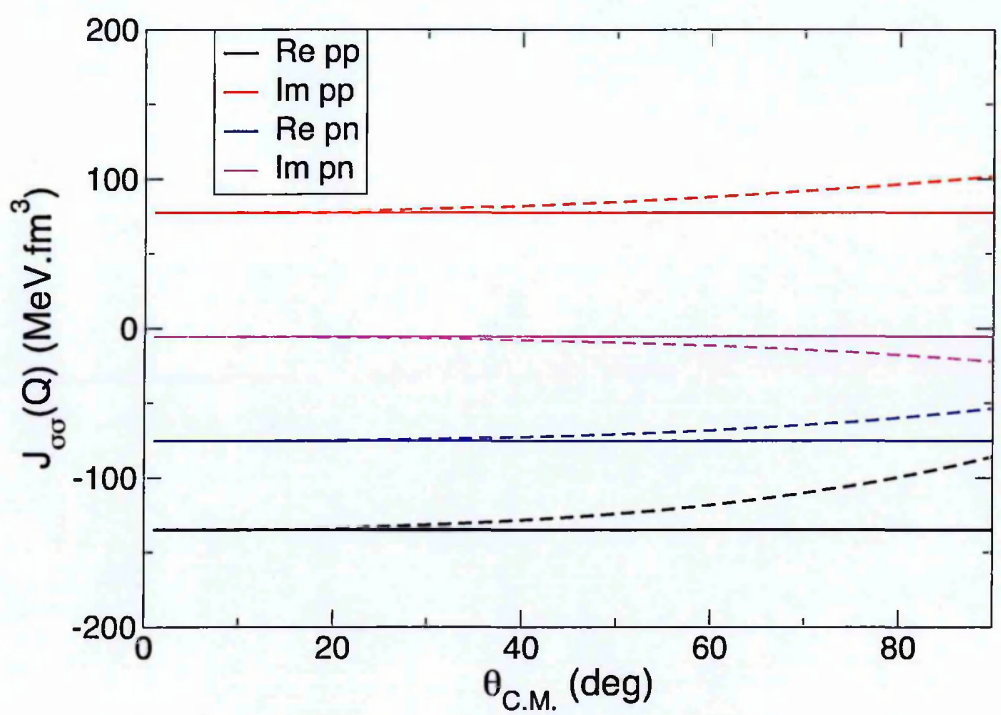

(a) Central exchange term coefficient

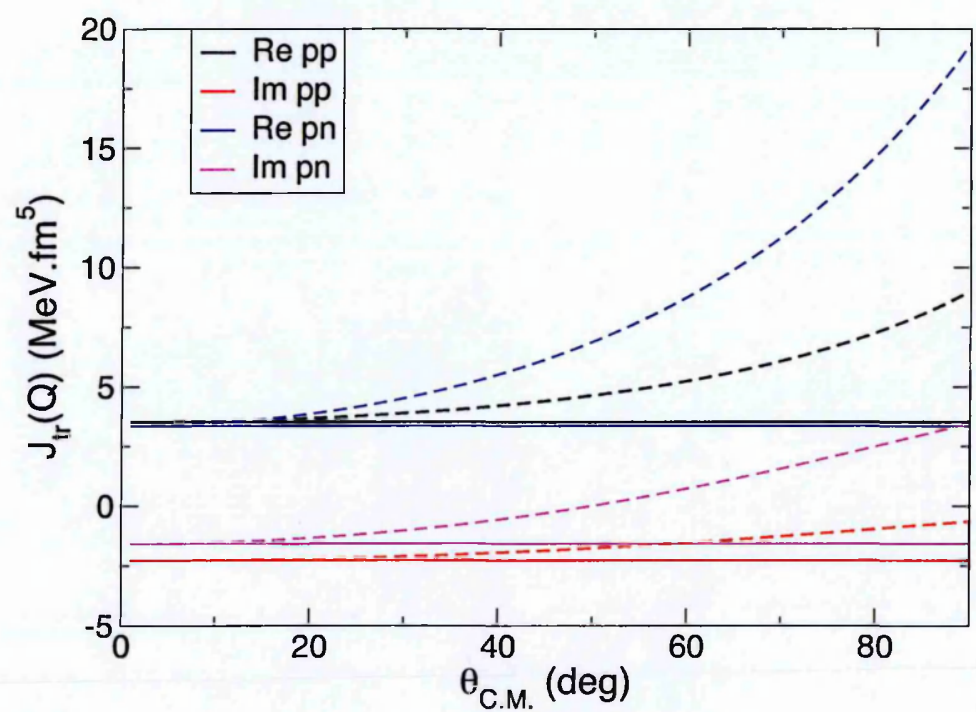

(b) Tensor exchange term coefficient

Figure 6.17: The exchange coefficients $\hat{J}_{\sigma \sigma}(Q)$ and $\hat{J}_{t r}(Q)$ for $Q=k_{c m}$ (solid lines) and $Q=k_{c m} \cos (\theta / 2)$ (dashed curves) for like 'pp' and unlike 'np' NN interactions.

calculated from the different types of spin-spin interaction terms plotted in Fig. 6.10 (from the folding of the direct terms), Fig. 6.12 (from the folding of the central exchange term) and Fig 6.16 (from the folding of the tensor exchange term). The cancellation means the deviation if $D_{N N}$ from unity shown in Figure 6.18 for all the spin-spin interactions combined is actually smaller than for some individual spin-spin interactions.

As has been noted previously in this chapter, the use of the WS single-particle wavefunction in the calculation of $D_{N N}$ leads to larger $1-D_{N N}$ at larger angles than the calculation using the $\mathrm{HO}$ wavefunction. The second trough in $D_{N N}$ (just below $70^{\circ}$ ) is 


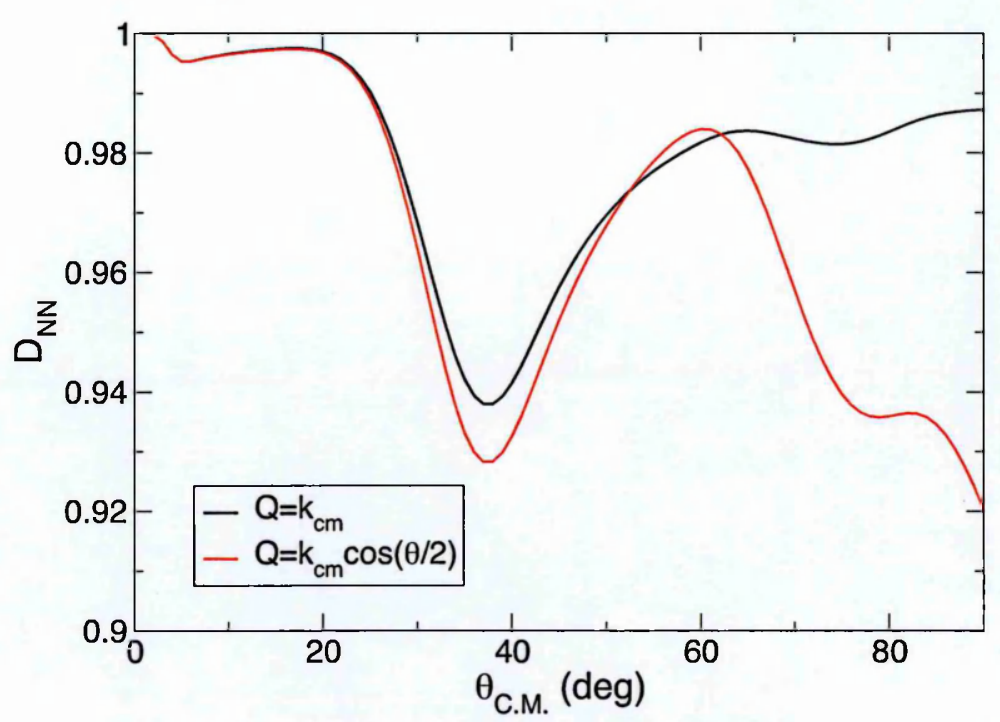

(a) HO single-particle wavefunction

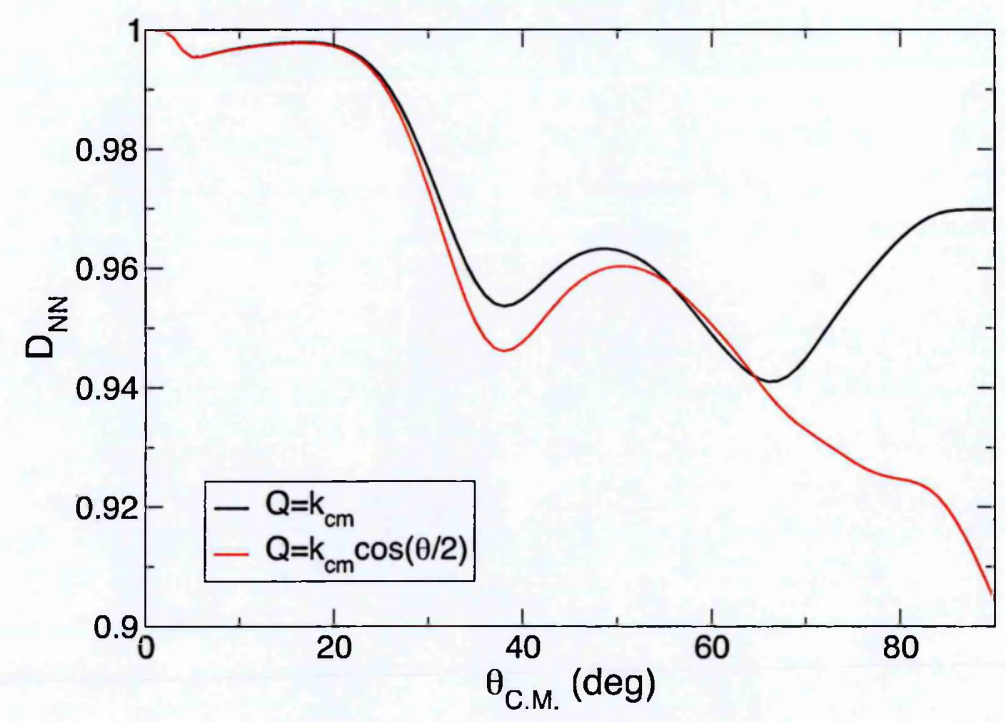

(b) WS single-particle wavefunction

Figure 6.18: $D_{N N}$ for elastic proton scattering from ${ }^{10} \mathrm{~B}$, calculated using all spin-spin interactions from folding of the direct and exchange central and tensor terms in the NN interaction. Spin-spin interactions calculated using (a) HO and (b) WS single-particle wavefunctions.

deeper in the black curve in Figure 6.18(b) than the first trough just below $40^{\circ}$. However, the black $D_{N N}$ curve for the HO wavefunction in Figure 6.18(a) has no significant deviation of $D_{N N}$ from unity around $70^{\circ}$.

Changing the approximation for $Q$ from $Q=k_{c m}$ to $Q=k_{c m} \cos (\theta / 2)$ also has a significant effect at large angles. Figure 6.18 shows that the black and red curves begin to deviate from one another at around $20^{\circ}$ which is the angle where the exchange coefficients $\hat{J}_{\sigma \sigma}(Q)$ and $\hat{J}_{t r}(Q)$ for the two different approximations of $Q$ also begin to deviate from 
one another in Figure 6.17. The shape of the black and red curves in Figure 6.18 have the same shape until around $60^{\circ}$ when the black curves $\left(Q=k_{c m}\right)$ move to smaller $1-D_{N N}$ and the red curves $\left(Q=k_{c m} \cos (\theta / 2)\right)$ move to larger $1-D_{N N}$. At $90^{\circ}$, in Figure 6.18(a), $D_{N N}\left(Q=k_{c m}\right)=0.987$ and $D_{N N}\left(Q=k_{c m} \cos (\theta / 2)\right)=0.920$, and in Figure 6.18(b), $D_{N N}\left(Q=k_{c m}\right)=0.970$ and $D_{N N}\left(Q=k_{c m} \cos (\theta / 2)\right)=0.904$. This signifies the importance of the approximation for $Q$ on the calculation of an accurate $D_{N N}$ at large angles.

To see how the deviation of $D_{N N}$ from unity is related to the strengths of all the spin-spin interactions, the ratio of $1-D_{N N}$ for $F_{k_{I} k}(R)$ and $0.5 \times F_{k_{I} k}(R)$ is plotted in Figure 6.19. In agreement with Figs. 6.6 and 6.9, when the strength of the spin-spin potential changes by a factor of 2 the deviation of $D_{N N}$ from 1 changes by a factor of 4. The dependence of $1-D_{N N}$ on the strength of the spin-spin potentials is a second order one due to the fact that only terms with the same rank in $\boldsymbol{I}$ can interfere with each other. This brings into question whether is is appropriate to use the first order DWBA to calculated the spin-spin amplitudes. This will be discussed further in the next chapter.

\subsection{Sensitivity of $D_{N N}$ to $u_{\ell j}(r)$}

The potential parameters (from [93]) used to calculate the WS single-particle wavefunction discussed in Section 6.1 have significant error bars, $R_{W S}=(0.88 \pm 0.13)(A-1)^{1 / 3} \mathrm{fm}$ and $a=0.81 \pm 0.08 \mathrm{fm}$. To examine the sensitivity of $D_{N N}$ to $u_{1 \frac{3}{2}}(r)$ the wavefunctions were calculated using the four combinations of the maximum and minimum values for

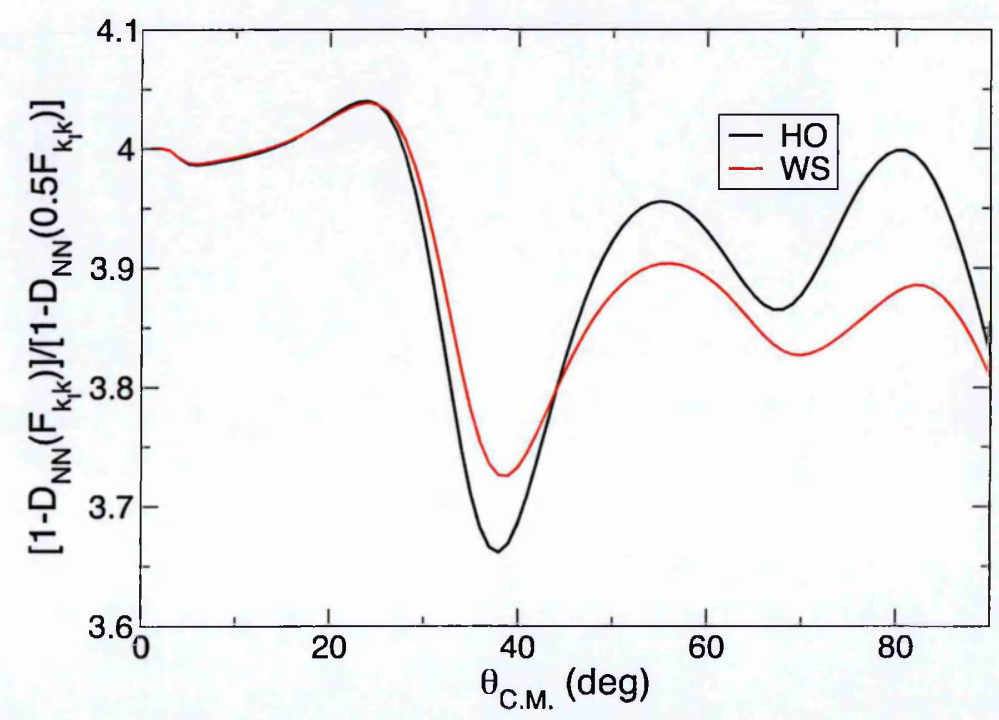

Figure 6.19: Ratio of the deviation of $D_{N N}$ from unity, including all the spin-spin interactions $F_{k_{I} k}(R)$ and $0.5 F_{k_{I} k}(R)$, direct and exchange, for elastic $\mathrm{p}+{ }^{10} \mathrm{~B}$ scattering. 
$R_{W S}$ and $a$. The maximum values for the radius $R_{W S}=(0.88+0.13)(A-1)^{1 / 3} \mathrm{fm}$ and diffuseness $a=0.81+0.08 \mathrm{fm}(\operatorname{Max})$ and the minimum values for the radius $R_{W S}=$ $(0.88-0.13)(A-1)^{1 / 3} \mathrm{fm}$ and diffuseness $a=0.81-0.08 \mathrm{fm}(\mathrm{Min})$, are used to calculate the two extremes in single-particle wavefunctions and their derivatives, shown in Figure 6.20 .

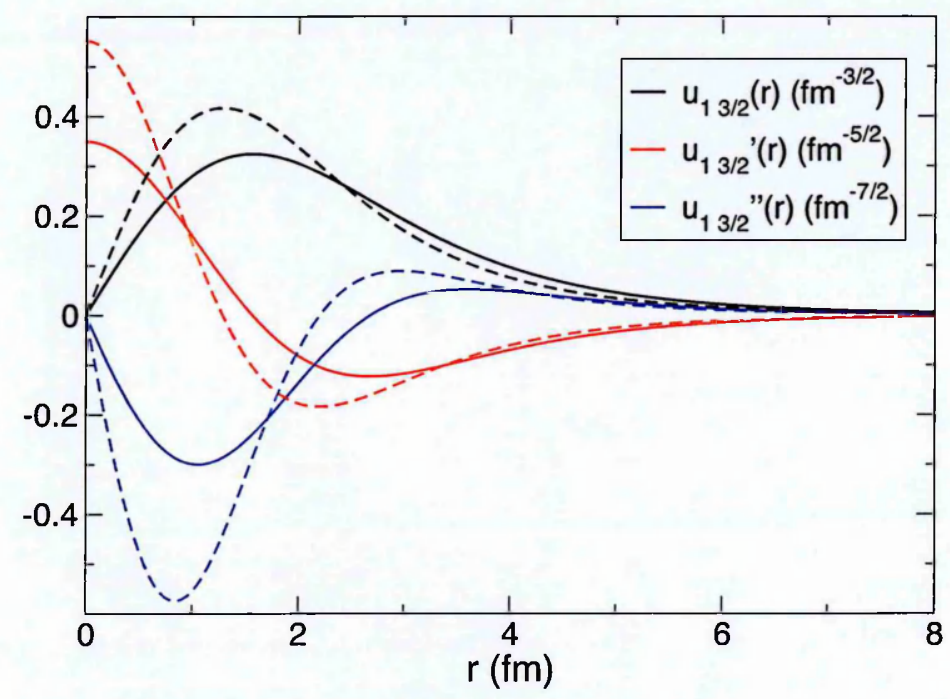

(a) Valence neutron

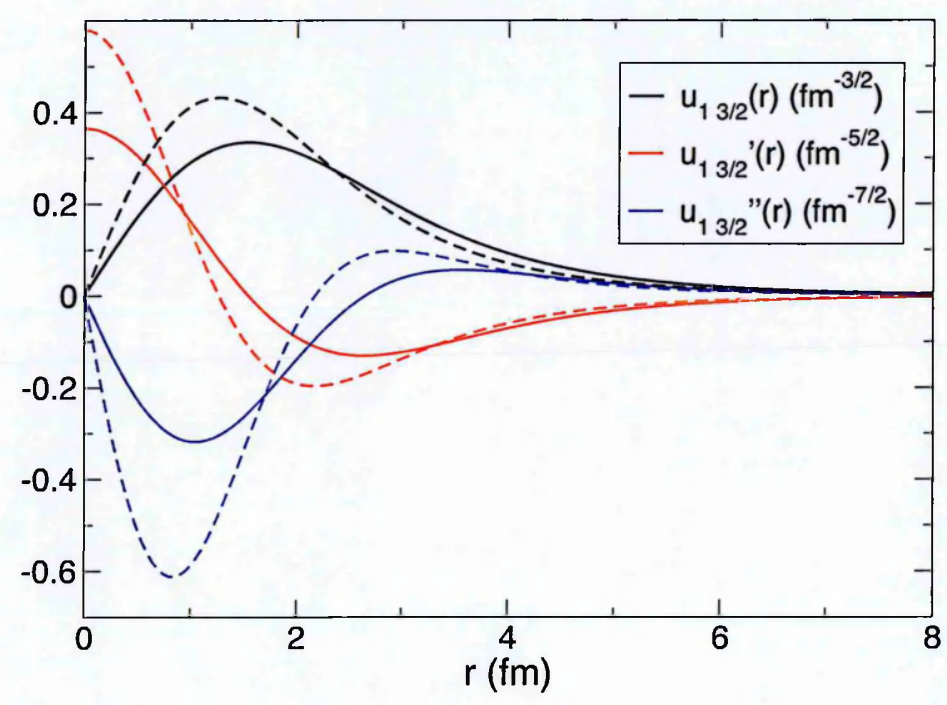

(b) Valence proton

Figure 6.20: The $1 p_{\frac{3}{2}}$ Woods-Saxon single-particle wavefunction with geometry parameters from [93] $R_{W S}=(0.88 \pm 0.13)(A-1)^{1 / 3} \mathrm{fm}$ and $a=0.81 \pm 0.08 \mathrm{fm}$, for (a) valence neutron and (b) valence proton. The red and blue curves are the first and second derivative of $u_{1 \frac{3}{2}}(r)$ respectively. The solid lines are for parameters $R_{W S}=(0.88+0.13)(A-1)^{1 / 3} \mathrm{fm}$ and $a=0.81+0.08 \mathrm{fm}(\mathrm{Max})$ and the dashed lines are for $R_{W S}=(0.88-0.13)(A-1)^{1 / 3}$ fm and $a=0.81-0.08 \mathrm{fm}$ (Min). 
Using the parameter set, Min leads to a higher peak in $u_{1 \frac{3}{2}}(r)$ at lower $r$ than the Max parameter set. The first and second derivatives are also significantly different for the two different sets of WS parameters. The curve of the first derivative is much steeper and the negative trough in the second derivative is much deeper for the Min parameters compared to the Max. The tail of $u_{1 \frac{3}{2}}(r)$ is also shallower for Min.

The calculation of $D_{N N}$ using the four different sets of wavefunctions is shown in Figure 6.21. At large angles there is a very large difference between the $D_{N N}$ curves calculated using the Max and Min parameters. $D_{N N}$ depends not only on the singleparticle wavefunction but also on the wavefunctions first and second derivatives through the tensor exchange terms. This makes this polarisation observable very sensitive to changes in the shape of the wavefunction $u_{1 \frac{3}{2}}(r)$. This sensitivity may make $D_{N N}$ a potentially useful probe of nuclear structure.

\subsection{Comparison with Experiment}

Figure 6.22 shows the calculations of $D_{N N}$ using four different single-particle wavefunctions. The black and dark blue curves use a WS wavefunction with the Max and Min parameters respectively. The red curve uses the $\mathrm{HO}$ wavefunction and the cyan curve the WS wavefunction discussed in Section 6.1. The circular points are the measurements of $D_{N N}$ for $200 \mathrm{MeV}$ protons elastically scattering from ${ }^{10} \mathrm{~B}$, captured from Figure 4 in [35].

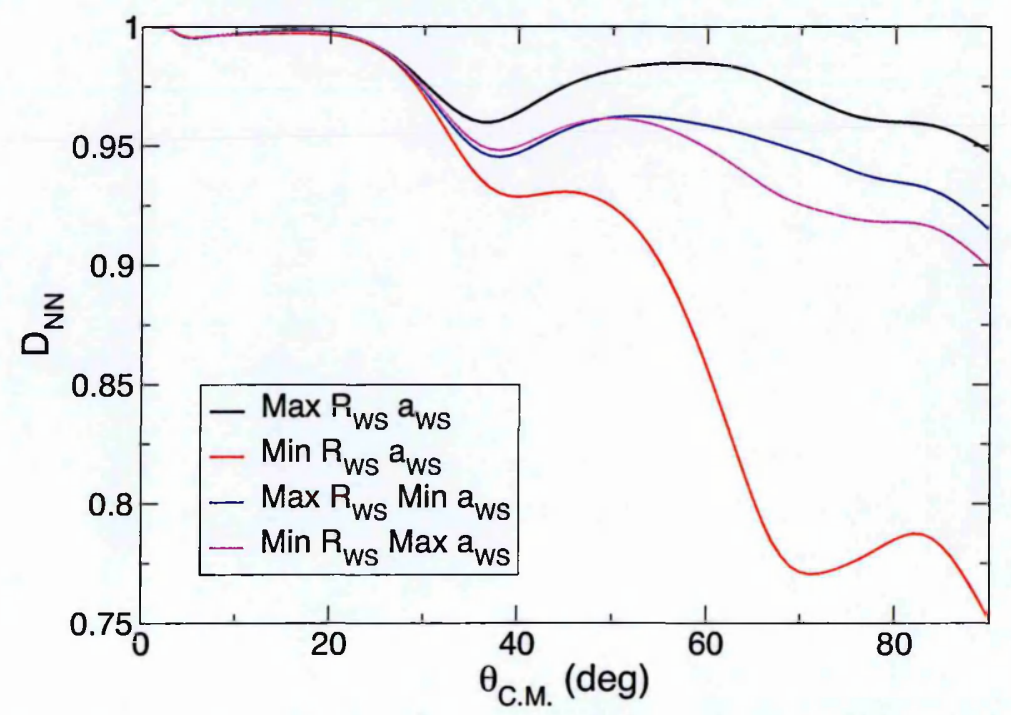

Figure 6.21: Calculation of $D_{N N}$, for elastic proton scattering from ${ }^{10} \mathrm{~B}$ using WoodsSaxon single-particle wavefunction with $\operatorname{Max} R_{W S}=(0.88+0.13)(A-1)^{1 / 3} \mathrm{fm}$ and $a=0.81+0.08 \mathrm{fm}$ and Min $R_{W S}=(0.88-0.13)(A-1)^{1 / 3} \mathrm{fm}$ and $a=0.81-0.08 \mathrm{fm}$ geometry parameters from [93]. 


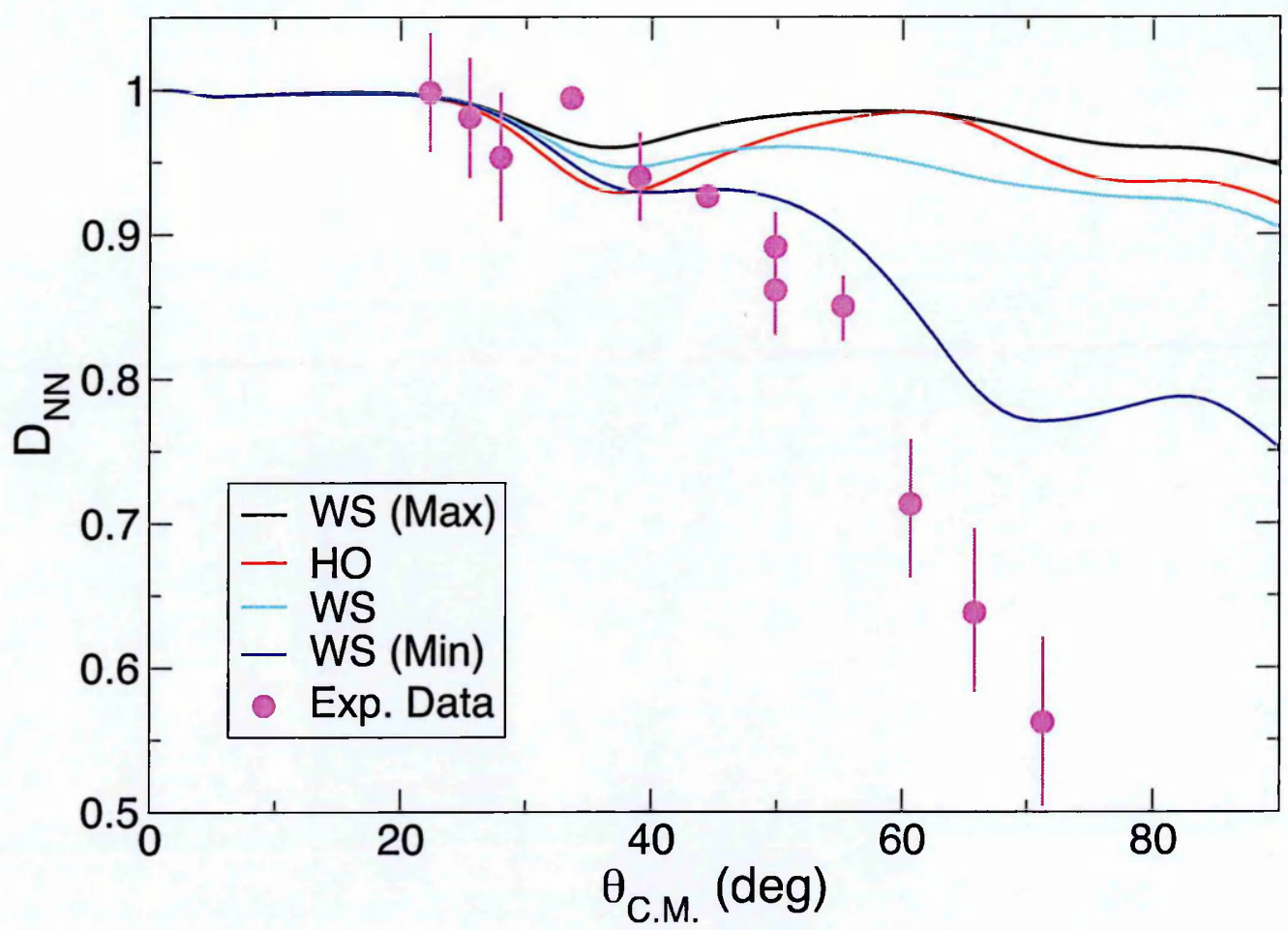

Figure 6.22: Calculation of $D_{N N}$, for elastic proton scattering from ${ }^{10} \mathrm{~B}$ using $\mathrm{HO}$ and WS single-particle wavefunctions with geometry parameters from [93] $R_{W S}=(0.88 \pm$ $0.13)(A-1)^{1 / 3} \mathrm{fm}$ and $a=0.81 \pm 0.08 \mathrm{fm}$ and the experimental measurement captured from Fig. 4 of [35]

Even the calculation of $D_{N N}$ using the Min WS parameters, which has the largest deviation of $D_{N N}$ from one, does not reproduce the experimental data at large angles. Of the approximations used in this work to enable the calculation of $D_{N N}$ using the spin-spin interactions, the simplification of the nuclear wavefunction, detailed in Section 3.3.1, is a significant limiting factor on the calculation. Further work needs to be done on incorporating a more realistic ${ }^{10} \mathrm{~B}$ wavefunction into this calculation as well as confirmation of the experimental data before it can be determined if this deviation of $D_{N N}$ from unity can be explained solely using spin-spin terms in the scattering potential. This will be discussed further in the next chapter.

\subsection{Computer Codes}

The computer codes used to calculate the spin-spin interactions and the DWBA amplitudes for protons elastically scattered from ${ }^{10} \mathrm{~B}$ were written by the author using FORTRAN 90. All stages of the calculation were checked using the computational software program Mathematica. In addition existing FORTRAN codes were used to calculate the 
distorted wave and single particle radial wavefunctions. The program used to generate the S-matrix elements and distorted radial wave functions for scattering in a complex potential was modified from a routine provided by Prof J. A. Tostevin. The code used to calculate the single particle wavefunctions by solving the Schrödinger equation for bound states has been contributed to my numerous members of the nuclear theory group at the University of Surrey. FORTRAN codes were also used to calculate the spherical Bessel functions, written by Prof I. J. Thompson and the angular momentum coefficients, provided by Prof J. A. Tostevin (multiple authors). 


\section{Chapter 7}

\section{Conclusions and Outlook}

\subsection{Conclusions}

A general model has been developed for spin-spin terms in the elastic nucleon-nucleus optical potential. The spin-spin potentials are generated by folding a realistic effective NN interaction over the nuclear wavefunction. The target is assumed to have a two valence-particle structure with the total spins of the two valence nucleons coupling to give the total spin of the nucleus. This general model has been used for the specific calculation of the elastic scattering of protons from ${ }^{10} \mathrm{~B}$.

One of the main goals of this work was to investigate the different types of spin-spin interaction and their effect on $D_{N N}$. The spin-spin interactions of the form $U_{k_{I} k}(\boldsymbol{R})=$ $F_{k_{I} k}(R) S_{k_{I} k}$, derived from the folding model included the spherical, $U_{10}(R)$, and tensor, $U_{12}(\boldsymbol{R})$, potentials, used in the first studies on spin-spin interactions [19]. If the projectile and target are spin- $-\frac{1}{2}$, these are the only local spin-spin potentials allowed by parity, time reversal and angular momentum conservation.

The ${ }^{10} \mathrm{~B}$ nucleus has a $3^{+}$ground state spin and parity. In the simple model of ${ }^{10} \mathrm{~B}$, the valence proton and neutron (both in $1 p_{\frac{3}{2}}$ shells) couple together to give the total spin of the nucleus. The $9 \mathrm{j}$ coefficient in the general folding expression, Eq. 3.23, shows that the total angular momenta of the valence nucleons $j_{1}$ and $j_{2}$, also couple to give the maximum allowed rank, $k_{I}=3$, of the target spin $\boldsymbol{I}$. This means that McAbee's $U_{32}(\boldsymbol{R})$ potential $[20,21]$ as well as the new $U_{34}(\boldsymbol{R})$ spin-spin potential are also derived from this folding model. Of these four spin-spin potentials, $U_{10}(R)$ and $U_{32}(\boldsymbol{R})$ have been shown to have the most significant effect on $D_{N N}$.

Comparing the deviations of $D_{N N}$ from unity caused by spin-spin interactions derived from the direct and exchange terms in the NN interaction show that they both contribute significantly. Figure 6.12 shows that the central exchange terms actually leads to the largest magnitude for $1-D_{N N}$, but cancellation between the spin-spin potentials of the 
central direct and exchange terms diminishes the deviation of $D_{N N}$ from unity when both are included. Of the tensor exchange terms, Term B, (where the grad operators of the solid spherical harmonic act on the initial distorted wavefunction), causes the most significant deviation of $D_{N N}$ from unity. The contribution of tensor exchange has been neglected from all previous work on spin-spin interactions. We have found that the effects on $D_{N N}$ of these spin-spin potentials are not negligible.

More generally, which spin-spin terms are present and how strong they are depends directly on the structure of the target. It has already been stated that the allowed rank of the target spin, $k_{I}$, is determined by the coupling of the total spins of the valence nucleons. The rank $k$ of the spherical harmonic, $Y_{k}(\hat{\boldsymbol{R}})$, in the generalised local spin-spin operator (Eq. 2.1) is dependent on the orbital angular momentum of the levels occupied by the valence nucleons, although it is only a simple coupling for the central terms. For valence nucleons in shells with larger orbital angular momentum, $\ell$, and total spin, $j$, higher order spin-spin operators will be allowed by this model.

In Section 6.6 it was shown that, at large angles $D_{N N}$ is very sensitive to the single particle wavefunction. Figure 6.22 shows the calculation of $D_{N N}$ for four different types of single-particle wavefunction compared to experiment. The WS wavefunction is calculated using a WS potential with parameters $R_{W S}=(0.88 \pm 0.13)(A-1)^{1 / 3} \mathrm{fm}$ and $a=0.81 \pm 0.08$ $\mathrm{fm}$ taken from [93]. Taking the maximum radius and diffuseness allowed by the error bars leads to a significantly different deviation of $D_{N N}$ from unity compared with using the minimum values (see Figure 6.21). The figures of $D_{N N}$ throughout Chapter 6 also show consistent differences between calculations using the $\mathrm{HO}$ and WS wavefunctions discussed in Section 6.1. The sensitivity of the spin-spin interactions and their effect on $D_{N N}$ to the occupation of different orbitals and their radial wavefunctions means that their effects on polarisation observables could be potentially used as a probe of nuclear structure.

The single-particle model of the target nucleus used in this work is very simplistic. This will limit the application of this model to other nucleon-nucleus interactions as many nuclei are not well described by the single-particle model. Configuration mixing complicates the picture of the nucleus by introducing admixtures of different valence orbital angular momenta, as well as non-zero spin cores. We have chosen to focus this work on the investigation of the different spin-spin interactions and their effect on the polarisation transfer coefficient $D_{N N}$ and not attempted to incorporate a more realistic wavefunction. More complete calculations of this nature are required to obtain a balance between the treatment of a sufficient number of spin-spin operators, to accurately describe the target spin-dependent nucleon-nucleus interaction, and a realistic treatment of the target wavefunction.

The simple nuclear model used in this work is also a limiting factor when comparing 
the final calculation of $D_{N N}$ with the experimental data. Figure 6.22 shows that none of the calculations, using different single-particle wavefunctions, reproduce the experimental data from [35] at large angles. However, the calculation of $D_{N N}$ is not only sensitive to the single particle wavefunction at large angles. Figure 6.18 also shows the approximation for $Q$ has a considerable effect on $D_{N N}$ at large angles. Here the deviation of $D_{N N}$ from unity is measured to be greatest [35] and the calculation of $Q$ is therefore important for the comparison of this model with experimental data. Before we can look beyond spinspin interactions for another explanation for the large measured deviation of $D_{N N}$ from unity, further work needs to be done on incorporating a more realistic ${ }^{10} \mathrm{~B}$ wavefunction into the calculation, as well as better approximation for $Q$ to more accurately determine the exchange terms.

One limitation of using $D_{N N}$ to study spin-spin interactions is that this observable has a second order dependence on the strength of the interaction. This is shown in Figure 6.19 and is due to the fact that for this observable only terms in the scattering amplitude with the same rank, $k_{I}$, of the target spin, $\boldsymbol{I}$, can interfere with each other, as discussed in Section 6.4.2. This brings into question the validity of using the first order DWBA to calculate the spin-spin amplitude as this approximation discards other second order terms. It may also indicate that to investigate the effect of spin-spin interactions on $D_{N N}$, a higher order calculation is required, possibly using a coupled channels approach, which has been used in the past to study spin-spin interactions $[4,6,7,17,18]$.

Alternatively, to study first order effects of spin-spin interactions another observable could be investigated. $D_{N N}$ is only dependent on the polarisation of the projectile nucleon. An observable that is dependent on the polarisation of the target would lead to a first order dependence on the strength of the spin-spin interactions. In Appendix B the trace of the cross product of the spin-zero amplitude and the spherical spin-spin amplitude is shown to always be zero. If instead of $\operatorname{Tr}\left[F \sigma_{0 y} F^{\dagger} \sigma_{0 y}\right]$ the observable was dependent on $\operatorname{Tr}\left[F \sigma_{0 y} F^{\dagger} I_{y}\right]$ this cross term would survive. Such an observable would be a more sensitive probe of spin-spin interactions.

In conclusion, a folding model has been used to construct new spin-spin tensor interactions within a simple two valence-particle model for the target nucleus, using a realistic nucleon-nucleon interaction that includes direct and exchange terms. This has led to higher order couplings of the target and projectile spins, to be incorporated into this calculation. In addition, spin-spin interactions from non-local tensor exchange contributions to the NN interaction have also been included for the first time.

It has been found that the spin-spin interactions derived from this model cause a significant deviation of $D_{N N}$ from unity when used to examine the elastic scattering of protons from ${ }^{10} \mathrm{~B}$. However, at large angles the calculation does not match the experimen- 
tal data from [35]. The calculation of $D_{N N}$ has been shown to be particularly sensitive to the structure of the nucleus and the simple nuclear model adopted is a definite limiting factor when comparing the model to experiment.

While this polarisation transfer coefficient was identified by Stamp to be the simplest way of experimentally verifying the existence of spin-spin interactions [7], the second order dependence of $D_{N N}$ on the strength of the spin-spin potentials may mean other polarisation observables provide a better method to study them.

\subsection{Outlook}

The model developed in this work has so far only been applied to the specific case of elastic proton scattering from ${ }^{10} \mathrm{~B}$. To make the model more widely applicable it would be useful to generalise it to apply to other non-zero spin nuclei that can be modelled as one or two valence particle systems. Part of this generalisation should include a reconstruction of the formalism to allow different wavefunctions to be used in the folding calculation, so more realistic wavefunctions can be used to describe the state of the nucleus. This work has highlighted that the spin-spin interactions and their effect on the deviation of the polarisation coefficient $D_{N N}$ from unity is highly sensitive to the parameters of the model which determine the size and structure of the nucleus. Incorporating a more physical wavefunction that accurately describes the properties of the target nucleus should therefore be a priority for future work.

In addition, the current effective $\mathrm{NN}$ interaction between the incident proton and valence particles is for free NN scattering. For a projectile energy of $200 \mathrm{MeV}$ this is a sensible starting point, but it is unclear how a density dependent NN interaction would affect the calculation of spin-spin interactions. In folding models a local density approximation is often used [44]. This assumes that the radial dependence of the interaction is independent of density and so the density dependent interaction has the factored form, $D(\rho) v_{N N}(r)$, [74]. McAbee discussed this approximation in his work [20,21], but neglected it in his final calculation. A study of how the density dependence of the NN interaction affects spin-spin interactions therefore still remains to be performed.

The approximation for $Q$ used to calculate the spin-spin interactions from the folding of the tensor exchange terms has been shown to have a significant effect on $D_{N N}$ at large angles. The expression $Q=k_{c m} \cos (\theta / 2)$ used in this work is derived using the assumption that the valence nucleon is bound to a core of infinite mass. The prescription for $Q$ in [71], (which tends to $Q=k_{c m} \cos (\theta / 2)$ in the limit $A \rightarrow \infty$ ), gives an $A$ dependent approximation for $Q$. As $D_{N N}$ is so sensitive to the approximation for $Q$ at large angles it would be interesting to see how this $A$-dependence affects the calculation of this 
observable.

Spin-spin interactions from the folding of the spin-orbit term in the NN interaction have been discussed in this work, although they have not been included in the calculation. McAbee found that inclusion of spin-spin interactions from the folding of the spin-orbit term had a negligible effect on spin observables [20,21]. However, he only examined terms that coupled $\boldsymbol{\sigma}_{0}, \boldsymbol{I}$ and $\boldsymbol{R}$ and neglected non-local terms with a momentum dependence. The folding of the tensor exchange term in the NN interaction lead to local (Term A) and non-local (Term B and Term C) spin-spin interactions. The effect on $D_{N N}$ of the local term shown by the black curve in Figure 6.16 is negligible and it is the non-local terms which cause $D_{N N}$ to deviate significantly from unity. A more comprehensive study of spin-spin interactions from the folding of the spin-orbit term in the NN interaction including momentum dependent terms is therefore still needed.

Spin-spin potentials from the spin-orbit term may also influence other observables. An experimental measurement of the analysing power is given in Betker et al. [35] along with the measurement of $D_{N N}$ for protons elastically scattered from ${ }^{10} \mathrm{~B}$. Their optical model calculation, which does quite well at reproducing the ratio-to-Rutherford cross section, fails to reproduce the analysing power at large angles. It is possible that an investigation into the spin-spin interactions from the folding of the spin-orbit term in the NN interaction may explain why the analysing power cannot be described by standard terms in the optical potential.

Until the improvements to the model outlined in this section have been made, it is difficult to determine for sure if the experimental measurement of $D_{N N}$, examined in this work, can be explained solely using spin-spin interactions. Confirmation of the experimental data is required, along with further development of the theoretical model, to ascertain the cause of the large measured deviation of $D_{N N}$ from unity at large scattering angles.

The best way to further study spin-spin interactions is not necessarily to take more measurements of $D_{N N}$. The second order dependence of $D_{N N}$ on the strengths of the spin-spin interactions means that not only is this observable less sensitive to target-spin dependent potentials, but that first order potential scattering approximations like DWBA may not be applicable. To better investigate spin-spin interactions, other observables with a first order dependence on the strengths, such as those dependent on the polarisation of the target spin, should be used. A better understanding of these spin-spin terms in the optical potential, will enable their sensitivity to the form of the nuclear wavefunction and their effect on polarisation observables to be used as a probe of nuclear structure. 


\section{Appendix A}

\section{Angular Momentum}

\section{A.1 Clebsch-Gordan Coefficient}

The vector addition coefficients that describe the coupling of two angular momenta are represented by $\left(j_{1} m_{1} j_{2} m_{2} \mid J M\right)$, where $m_{1}, m_{2}$ and $M$ are the magnetic quantum numbers of the component angular momenta $j_{1}$ and $j_{2}$ and the total angular momentum $J$, respectively $[42,48]$. These coefficients are sometimes called Clebsch-Gordan (or Wigner) coefficients and will be referred to as C-G coefficients in the text. The C-G coefficient is zero unless $M=m_{1}+m_{2}$ and the values of $J$ are restricted by the 'triangle condition' $j_{1}+j_{2} \geqslant J \geqslant\left|j_{1}-j_{2}\right|$.

The orthogonality relations for the C-G coefficients are [42]

$$
\begin{aligned}
\sum_{m_{1} m_{2}}\left(j_{1} m_{1} j_{2} m_{2} \mid J M\right)\left(j_{1} m_{1} j_{2} m_{2} \mid J^{\prime} M^{\prime}\right) & =\delta_{J, J^{\prime}} \delta_{M, M^{\prime}} \\
\sum_{J M}\left(j_{1} m_{1} j_{2} m_{2} \mid J M\right)\left(j_{1} m_{1}^{\prime} j_{2} m_{2}^{\prime} \mid J M\right) & =\delta_{m_{1}, m_{1}^{\prime}} \delta_{m_{2}, m_{2}^{\prime}}
\end{aligned}
$$

where $\delta_{J, J^{\prime}}$ is unity if $J=J^{\prime}$ and is zero if $J \neq J^{\prime}$. The symmetry relations useful when manipulating $\mathrm{C}-\mathrm{G}$ coefficients are

$$
\begin{aligned}
\left(j_{1} m_{1} j_{2} m_{2} \mid J M\right) & =(-)^{j_{1}+j_{2}-J}\left(j_{2} m_{2} j_{1} m_{1} \mid J M\right) \\
& =(-)^{j_{1}+j_{2}-J}\left(j_{1}-m_{1} j_{2}-m_{2} \mid J-M\right) \\
& =\frac{\hat{J}}{\hat{j_{2}}}(-)^{j_{1}-m_{1}}\left(j_{1} m_{1} J-M \mid j_{2}-m_{2}\right) \\
& =\frac{\hat{J}}{\hat{j_{1}}}(-)^{j_{2}+m_{2}}\left(J-M j_{2} m_{2} \mid j_{1}-m_{1}\right)
\end{aligned}
$$

where $\hat{J}$ is used to denote $\sqrt{2 J+1}$. These symmetry relations are also given in Appendix 


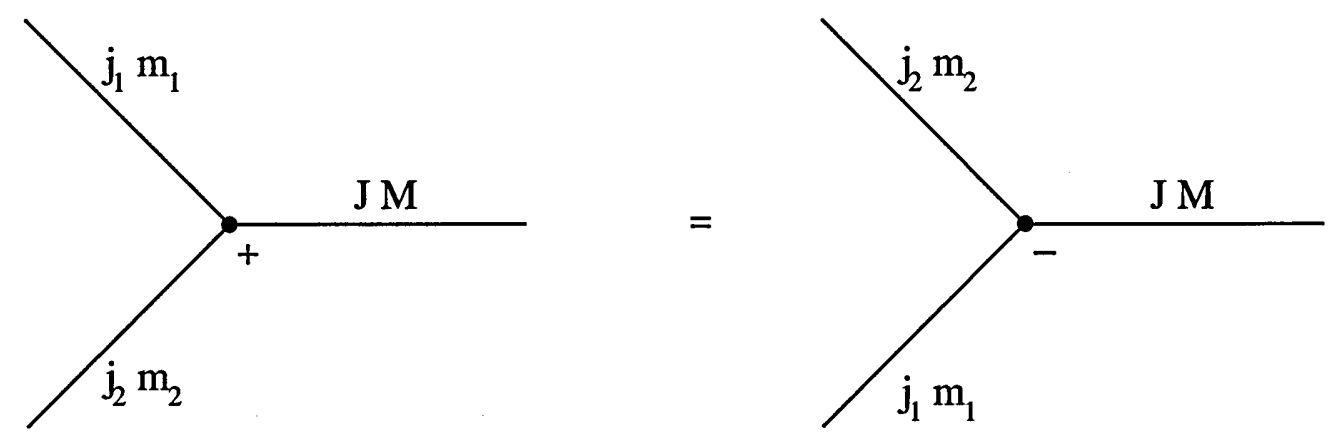

Figure A.1: The graphical representation of a C-G coefficient of the form $\left(j_{1} m_{1} j_{2} m_{2} \mid J M\right)$.

I of [42].

The graphical method in angular momentum, detailed in $[42,90]$, displays sums involving the product of $\mathrm{C}-\mathrm{G}$ coefficients more clearly and compactly than their algebraic form. When dealing with a large number of $\mathrm{C}-\mathrm{G}$ coefficients the graphical representation enables the interrelations between the quantum numbers and the structure of the expressions to be seen more easily. The $\mathrm{C}-\mathrm{G}$ coefficient itself is represented by a node junction of three solid lines as shown in Figure A.1. The orientation of the node is given by the sign. A + sign denotes an anti-clockwise orientation and a - sign denotes a clockwise orientation. For the purposes of this work, the graphical representation is mainly used to aid the reduction of sums of multiple C-G coefficients.

\section{A.2 Racah Coefficient}

The Racah coefficient W(abcd;ef) detailed in $[42,48]$, can be expressed as a useful contraction of four C-G coefficients

$$
\hat{e} \hat{f} W(a b c d ; e f) \delta_{c, c^{\prime}} \delta_{\gamma, \gamma^{\prime}}=\sum_{\alpha \beta \delta \epsilon \phi}(a \alpha b \beta \mid e \epsilon)(e \epsilon d \delta \mid c \gamma)(b \beta d \delta \mid f \phi)\left(a \alpha f \phi \mid c^{\prime} \gamma^{\prime}\right)
$$

where the Roman letters represent the angular momenta and the Greek letters depict their magnetic quantum numbers. Expressing the Racah coefficient in this way reveals the four (one for each C-G coefficient) triads of vectors which have to satisfy the 'triangular conditions': abe, edc, bdf and afc, otherwise the coefficient is zero.

Eq. A.7 can be rearranged by multiplying both sides of the equation by the C-G coefficient $(a \alpha f \phi \mid c \gamma)$ and summing over $c^{\prime} \gamma^{\prime}$. The orthogonality relationship given in Eq. A.1 can then be used to rearrange Eq. A.7 in the following way

$$
\hat{e} \hat{f} W(a b c d ; e f)(a \alpha f \phi \mid c \gamma)=\sum_{\beta \delta \epsilon}(a \alpha b \beta \mid e \epsilon)(e \epsilon d \delta \mid c \gamma)(b \beta d \delta \mid f \phi) .
$$




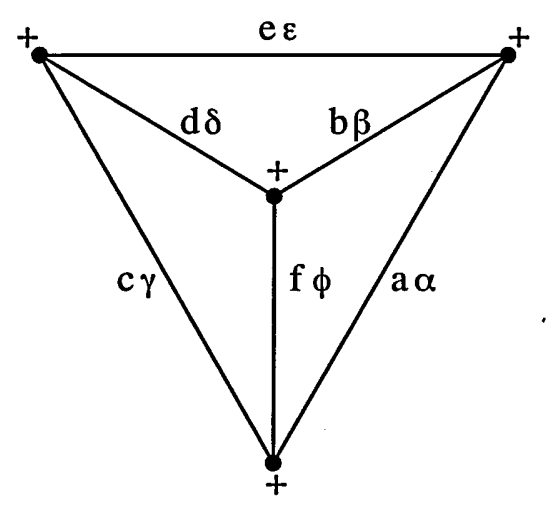

Figure A.2: The graph of the Racah coefficient, W(abcd;ef), adapted from Fig. 7.16 in [42].

This process can be repeated to move another C-G coefficient from the right hand side of Eq. A.7 to the left, as shown in Appendix II of [42].

The Wigner 6-j symbol is also used in this work and differs from the Racah coefficient in phase only

$$
W(a b c d ; e f)=(-)^{a+b+c+d}\left\{\begin{array}{lll}
a & b & e \\
d & c & f
\end{array}\right\} .
$$

The graphical representation of a Racah coefficient is shown in Fig. A.2.

\section{A.3 9-j Symbol}

Another coefficient used extensively in this work is the $9 \mathrm{j}$ symbol, the properties of which are described in $[42,94]$. The $9 \mathrm{j}$ symbol is denoted by

$$
\left\{\begin{array}{lll}
a & b & c \\
d & e & f \\
g & h & i
\end{array}\right\}
$$

It can be expressed in terms of six C-G coefficients in the following way [95]

$$
\begin{aligned}
\hat{e} \hat{f} \hat{c} \hat{c}^{\prime}\left\{\begin{array}{lll}
a & a^{\prime} & e \\
b & b^{\prime} & f \\
c & c^{\prime} & d
\end{array}\right\} \delta_{d, d^{\prime}} \delta_{\delta, \delta^{\prime}} & =\sum_{\alpha \alpha^{\prime} \beta \beta^{\prime} \gamma \gamma^{\prime} \epsilon \phi}(a \alpha b \beta \mid c \gamma)\left(a^{\prime} \alpha^{\prime} b^{\prime} \beta^{\prime} \mid c^{\prime} \gamma^{\prime}\right) \\
& \times(-)^{a^{\prime}-\alpha^{\prime}}\left(a \alpha a^{\prime}-\alpha^{\prime} \mid e \epsilon\right)(-)^{b^{\prime}-\beta^{\prime}}\left(b \beta b^{\prime}-\beta^{\prime} \mid f \phi\right) \\
& \times(-)^{c^{\prime}-\gamma^{\prime}}\left(c \gamma c^{\prime}-\gamma^{\prime} \mid d \delta\right)\left(e \epsilon f \phi \mid d^{\prime} \delta^{\prime}\right) .
\end{aligned}
$$


As with the relationship for the Racah coefficient given in Eq. A.7, Eq. A.11 can be rearranged by multiplying both sides by the $\mathrm{C}-\mathrm{G}$ coefficient $(e \epsilon f \phi \mid d \delta$ ) and summing over $d^{\prime} \delta^{\prime}$ to give

$$
\begin{aligned}
\hat{e} \hat{f} \hat{c} \hat{c}^{\prime}\left\{\begin{array}{lll}
a & a^{\prime} & e \\
b & b^{\prime} & f \\
c & c^{\prime} & d
\end{array}\right\}(e \epsilon f \phi \mid d \delta) & =\sum_{\alpha \alpha^{\prime} \beta \beta^{\prime} \gamma \gamma^{\prime}}(a \alpha b \beta \mid c \gamma)\left(a^{\prime} \alpha^{\prime} b^{\prime} \beta^{\prime} \mid c^{\prime} \gamma^{\prime}\right) \\
& \times(-)^{a^{\prime}-\alpha^{\prime}}\left(a \alpha a^{\prime}-\alpha^{\prime} \mid e \epsilon\right)(-)^{b^{\prime}-\beta^{\prime}}\left(b \beta b^{\prime}-\beta^{\prime} \mid f \phi\right) \\
& \times(-)^{c^{\prime}-\gamma^{\prime}}\left(c \gamma c^{\prime}-\gamma^{\prime} \mid d \delta\right),
\end{aligned}
$$

and, as with the Racah coefficient, this process can be repeated multiple times so that there are two C-G coefficients on the left hand side of Eq. A.12, then three, etc. (for details see Appendix III of [42]). The graphical representation of a $9 \mathrm{j}$ coefficient is shown in Fig. A.3.

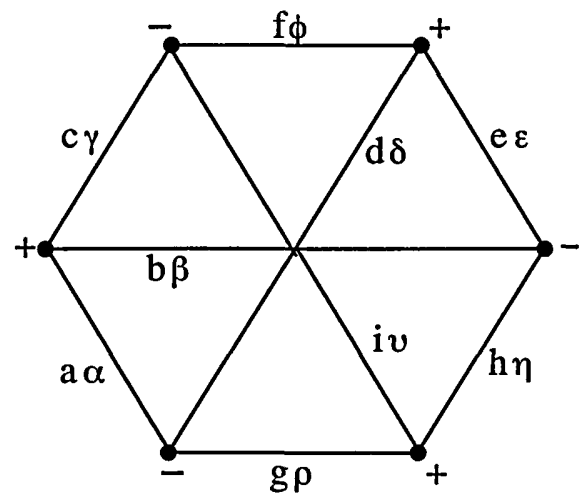

Figure A.3: The graph of the 9j coefficient shown in Eq. A.10.

\section{A.4 Spherical Harmonics}

The orbital angular momentum operator, $\ell$, is given by

$$
\ell=-\imath(r \wedge \nabla)
$$

Spherical harmonics are the eigenfunctions of the orbital angular momentum operator $\ell^{2}$ and $\ell_{z}$, which is the projection of $\ell$ along the z-axis. They describe the angular distribution of particles with orbital angular momentum, $\ell$ and projection, $m_{\ell}$, moving in a spherically symmetric field $\left\langle\theta, \phi \mid \ell, m_{\ell}\right\rangle=Y_{\ell m_{\ell}}(\theta, \phi)$. The set of normalised standard 
spherical harmonics used in this work are defined in [42] as

$$
\begin{aligned}
Y_{\ell m_{\ell}}(\theta, \phi) & =(-)^{m_{\ell}}\left[\frac{(2 \ell+1)}{4 \pi} \frac{\left(\ell-m_{\ell}\right) !}{\left(\ell+m_{\ell}\right) !}\right]^{1 / 2} P_{\ell}^{m_{\ell}}(\cos \theta) e^{i m_{\ell} \phi}, \quad m_{\ell} \geqslant 0 \\
Y_{\ell-m_{\ell}}(\theta, \phi) & =(-)^{m_{\ell}} Y_{\ell m_{\ell}}^{*}(\theta, \phi)
\end{aligned}
$$

where $P_{\ell}^{m_{\ell}}(\cos \theta)$ are the associated Legendre polynomials. There are two sign conventions for the associated Legendre polynomials so care must be taken when using them. In this work, the convention described in Section 12.5 of [96] is used.

Spherical harmonics are orthogonal over the unit sphere

$$
\int Y_{\ell^{\prime} m_{\ell}^{\prime}}^{*}(\theta, \phi) Y_{\ell m_{\ell}}(\theta, \phi) d \Omega=\delta_{\ell, \ell^{\prime}} \delta_{m_{\ell}, m_{\ell}^{\prime}}
$$

where $d \Omega=\sin \theta d \theta d \phi$. Two spherical harmonics with the same angular dependence can be combined in the following way

$$
Y_{\ell^{\prime} m_{\ell}^{\prime}}(\theta, \phi) Y_{\ell m_{\ell}}(\theta, \phi)=\frac{\hat{\ell}^{\prime} \hat{\ell}}{\sqrt{4 \pi}} \sum_{C \Gamma} \frac{Y_{C \Gamma}(\theta, \phi)}{\hat{C}}\left(\ell^{\prime} m_{\ell}^{\prime} \ell m_{\ell} \mid C \Gamma\right)\left(\ell^{\prime} 0 \ell 0 \mid C 0\right)
$$

Combining these two equations allows the combination and angular integration of three spherical harmonics in the following way

$$
\int Y_{\ell^{\prime} m_{\ell}^{\prime}}^{*}(\theta, \phi) Y_{k q}(\theta, \phi) Y_{\ell m_{\ell}}(\theta, \phi) d \Omega=\frac{\hat{k} \hat{\ell}}{\hat{\ell}^{\prime} \sqrt{4 \pi}}\left(k q \ell m_{\ell} \mid \ell^{\prime} m_{\ell}^{\prime}\right)\left(k 0 \ell 0 \mid \ell^{\prime} 0\right)
$$




\section{Appendix B}

\section{Simple Calculation of $D_{N N}$}

In Section 2.2.1 the polarisation transfer coefficient $D_{N N}$ was evaluated for the simple case where:

i) there is no spin-orbit term in the distorting potential and

ii) only the spherical spin-spin interaction is included.

This resulted in the simplified nucleon-nucleus elastic scattering amplitude given in Eq. 2.32. Substituting this into the top part of Eq. 1.3 for $D_{N N}$ gives

$$
\begin{gathered}
\operatorname{Tr}\left[F \sigma_{0 y} F^{\dagger} \sigma_{0 y}\right]=\operatorname{Tr}\left[|g(\theta)|^{2} \mathbf{1}+\left\{g(\theta) h_{10}^{*}(\theta)+g^{*}(\theta) h_{10}(\theta)\right\} \frac{\boldsymbol{\sigma}_{0} \cdot \boldsymbol{I}}{\sqrt{I(I+1)}}\right. \\
\left.+\left|h_{10}(\theta)\right|^{2} \frac{\left(\boldsymbol{\sigma}_{0} \cdot \boldsymbol{I} \sigma_{0 y}\right)^{2}}{I(I+1)}\right] .
\end{gathered}
$$

Taking each term in turn

$$
\begin{gathered}
\operatorname{Tr}\left[|g(\theta)|^{2} 1\right]=2(2 I+1)|g(\theta)|^{2} \\
\operatorname{Tr}\left[\left\{g(\theta) h_{10}^{*}(\theta)+g^{*}(\theta) h_{10}(\theta)\right\} \frac{\boldsymbol{\sigma}_{0} \cdot \boldsymbol{I}}{\sqrt{I(I+1)}}\right]=0
\end{gathered}
$$

because $\operatorname{Tr}\left[\sigma_{0 i} I_{j}\right]=0$, where $i$ and $j$ are $x, y$ or $z$. To determine the third term in Eq. B.1, $\operatorname{Tr}\left[\left(\boldsymbol{\sigma}_{0} \cdot \boldsymbol{I} \sigma_{0 y}\right)^{2}\right]$ must be evaluated

$$
\begin{aligned}
\operatorname{Tr}\left[\left(\boldsymbol{\sigma}_{0} \cdot \boldsymbol{I} \sigma_{0 y}\right)^{2}\right] & =\operatorname{Tr}\left[\left(-\imath \sigma_{0 z} I_{x}+I_{y}+\imath \sigma_{0 x} I_{z}\right)^{2}\right] \\
& =\operatorname{Tr}\left[-I_{x}^{2}+I_{y}^{2}-I_{z}^{2}\right] \\
& =\operatorname{Tr}\left[-\boldsymbol{I}^{2}+2 I_{y}^{2}\right] \\
& =\operatorname{Tr}\left[-I(I+1)+\frac{2}{3} I(I+1)\right] \\
& =2(2 I+1)\left[-\frac{1}{3} I(I+1)\right]
\end{aligned}
$$


Now the top part of Eq. 1.3 for $D_{N N}$ can be written as

$$
\operatorname{Tr}\left[F \sigma_{0 y} F^{\dagger} \sigma_{0 y}\right]=2(2 I+1)|g(\theta)|^{2}-2(2 I+1) \frac{1}{3}\left|h_{10}(\theta)\right|^{2} .
$$

The bottom part of Eq. 1.3 for $D_{N N}$ can be evaluated in a similar way to give

$$
\operatorname{Tr}\left[F F^{\dagger}\right]=2(2 I+1)|g(\theta)|^{2}+2(2 I+1)\left|h_{10}(\theta)\right|^{2} .
$$

Putting these parts together gives the expression for $D_{N N}$ shown in Eq. 2.33

$$
D_{N N}=\frac{\operatorname{Tr}\left[F \sigma_{0 y} F^{\dagger} \sigma_{0 y}\right]}{\operatorname{Tr}\left[F F^{\dagger}\right]}=\frac{|g(\theta)|^{2}-\frac{1}{3}\left|h_{10}(\theta)\right|^{2}}{|g(\theta)|^{2}+\left|h_{10}(\theta)\right|^{2}} .
$$




\section{Appendix $\mathrm{C}$}

\section{Folded Potential Formalism: Direct Terms}

In this Appendix the details will be given of the formalism used in the folding model described in Chapter 3 for the direct terms in the NN interaction.

\section{C.1 Folding of Central NN interaction Term}

Following on from the discussion in Section 3.5, the second central term in the NN interaction $v_{p p}^{\sigma \sigma}\left(\boldsymbol{r}_{p}\right) \boldsymbol{\sigma}_{0} \cdot \boldsymbol{\sigma}_{1}$, given by Eq. 3.28, can be folded over the target ground state wavefunction using the general folding Eq. 3.23, this gives

$$
\begin{aligned}
\left\langle\Psi_{I, M_{f}}\left(\boldsymbol{r}_{1}, \boldsymbol{r}_{2}\right)\left|v_{p p}^{\sigma \sigma}\left(r_{p}\right) \boldsymbol{\sigma}_{0} \cdot \boldsymbol{\sigma}_{1}\right| \Psi_{I, M_{i}}\left(\boldsymbol{r}_{1}, \boldsymbol{r}_{2}\right)\right\rangle \\
=(-)^{3 j_{1}-j_{1}^{\prime}} \hat{I} \hat{j}_{1} \hat{j}_{1}^{\prime} \int \sum_{\lambda_{1}^{\prime} \lambda_{1} m_{s 1}^{\prime} m_{s 1}} Y_{\ell_{1}^{\prime} \lambda_{1}^{\prime}}^{*}\left(\hat{\boldsymbol{r}}_{1}\right) \chi_{\frac{1}{2} m_{s 1}^{\prime}}^{*} u_{\ell_{1}^{\prime} j_{1}^{\prime}}\left(r_{1}\right) 4 \pi \sum_{K Q} \frac{f_{K}^{(\sigma \sigma)}\left(R, r_{1}\right)}{\hat{K}^{2}} \\
\quad \times Y_{K Q}(\hat{\boldsymbol{R}}) Y_{K Q}^{*}\left(\hat{\boldsymbol{r}}_{1}\right) \sum_{\mu_{0}}(-)^{\mu_{0}} \sigma_{1 \mu_{0}}^{(0)} \sigma_{1-\mu_{0}}^{(1)} Y_{\ell_{1} \lambda_{1}}\left(\hat{\boldsymbol{r}}_{1}\right) \chi_{\frac{1}{2} m_{s 1}} u_{\ell_{1} j_{1}}\left(r_{1}\right) d \boldsymbol{r}_{1} \\
\quad \times(-)^{1 / 2+m_{s 1}^{\prime}-\ell_{1}^{\prime}-\lambda_{1}} \sum_{k_{I} q_{I}} \hat{k}_{I}^{2} W\left(k_{I} j_{1} I j_{2} ; j_{1}^{\prime} I\right)\left(I M_{i} k_{I} q_{I} \mid I M_{f}\right) \sum_{k_{1} q_{1} k_{2} q_{2}}(-)^{k_{1}-k_{2} \hat{k}_{1}} \\
\quad \times\left(\frac{1}{2} m_{s 1} \frac{1}{2}-m_{s 1}^{\prime} \mid k_{1} q_{1}\right)\left(\ell_{1}-\lambda_{1} \ell_{1}^{\prime} \lambda_{1}^{\prime} \mid k_{2} q_{2}\right)\left(k_{1} q_{1} k_{I} q_{I} \mid k_{2} q_{2}\right)\left\{\begin{array}{ccc}
j_{1} & j_{1}^{\prime} & k_{I} \\
\frac{1}{2} & \frac{1}{2} & k_{1} \\
\ell_{1} & \ell_{1}^{\prime} & k_{2}
\end{array}\right\} .
\end{aligned}
$$


Coupling the three spherical harmonics with an angular dependence of $\hat{\boldsymbol{r}}_{1}$ and integrating over all angles using a rearranged form of Eq. A.17 gives

$$
\int d \Omega_{r_{1}} Y_{\ell_{1}^{\prime} \lambda_{1}^{\prime}}^{*}\left(\hat{\boldsymbol{r}}_{1}\right) Y_{K Q}^{*}\left(\hat{\boldsymbol{r}}_{1}\right) Y_{\ell_{1} \lambda_{1}}\left(\hat{\boldsymbol{r}}_{1}\right)=\frac{\hat{\ell}_{1}^{\prime}}{\sqrt{4 \pi}}(-)^{\ell_{1}^{\prime}-\lambda_{1}^{\prime}}\left(\ell_{1}-\lambda_{1} \ell_{1}^{\prime} \lambda_{1}^{\prime} \mid K-Q\right)\left(\ell_{1}^{\prime} 0 K 0 \mid \ell_{1} 0\right),
$$

and using the Wigner-Eckart theorem [42] to determine

$$
\begin{aligned}
\left\langle\frac{1}{2} m_{s 1}^{\prime}\left|\sigma_{1-\mu_{0}}^{(1)}\right| \frac{1}{2} m_{s 1}\right\rangle & =\sqrt{3}\left(\frac{1}{2} m_{s 1} 1-\mu_{0} \mid \frac{1}{2} m_{s 1}^{\prime}\right) \\
& =\sqrt{2}(-)^{1 / 2-m_{s 1}}\left(\frac{1}{2} m_{s 1} \frac{1}{2}-m_{s 1}^{\prime} \mid 1 \mu_{0}\right)
\end{aligned}
$$

gives the following

$$
\begin{aligned}
\left\langle\Psi_{I, M_{f}}\left(\boldsymbol{r}_{1}, \boldsymbol{r}_{2}\right)\left|v_{p p}^{\sigma \sigma}\left(r_{p}\right) \boldsymbol{\sigma}_{0} \cdot \boldsymbol{\sigma}_{1}\right| \Psi_{I, M_{i}}\left(\boldsymbol{r}_{1}, \boldsymbol{r}_{2}\right)\right\rangle & \\
= & (-)^{3 j_{1}-j_{1}^{\prime}} \sqrt{8 \pi} \hat{I} \hat{j}_{1} \hat{j}_{1}^{\prime} \hat{\ell}_{1}^{\prime} \int_{0}^{\infty} r_{1}^{2} d r_{1} u_{\ell_{1}^{\prime} j_{1}^{\prime}}\left(r_{1}\right) \sum_{K Q} \frac{f_{K}^{(\sigma \sigma)}\left(R, r_{1}\right)}{\hat{K}^{2}} u_{\ell_{1} j_{1}}\left(r_{1}\right) Y_{K Q}(\hat{\boldsymbol{R}}) \\
& \times \sum_{\mu_{0} \lambda_{1}^{\prime} \lambda_{1} m_{s 1}^{\prime} m_{s 1}} \sigma_{1 \mu_{0}}^{(0)}(-)^{\mu_{0}+1-m_{s 1}+m_{s 1}^{\prime}-\lambda_{1}-\lambda_{1}^{\prime}}\left(\frac{1}{2} m_{s 1} \frac{1}{2}-m_{s 1}^{\prime} \mid 1 \mu_{0}\right)\left(\ell_{1}-\lambda_{1} \ell_{1}^{\prime} \lambda_{1}^{\prime} \mid K-Q\right) \\
& \times\left(\ell_{1}^{\prime} 0 K 0 \mid \ell_{1} 0\right) \sum_{k_{I} q_{I} k_{1} q_{1} k_{2} q_{2}} \hat{k}_{I}^{2} W\left(k_{I} j_{1} I j_{2} ; j_{1}^{\prime} I\right)\left(I M_{i} k_{I} q_{I} \mid I M_{f}\right)(-)^{k_{1}-k_{2}} \\
& \times \hat{k}_{1}\left(\frac{1}{2} m_{s 1} \frac{1}{2}-m_{s 1}^{\prime} \mid k_{1} q_{1}\right)\left(\ell_{1}-\lambda_{1} \ell_{1}^{\prime} \lambda_{1}^{\prime} \mid k_{2} q_{2}\right)\left(k_{1} q_{1} k_{I} q_{I} \mid k_{2} q_{2}\right)\left\{\begin{array}{ccc}
j_{1} & j_{1}^{\prime} & k_{I} \\
\frac{1}{2} & \frac{1}{2} & k_{1} \\
\ell_{1} & \ell_{1}^{\prime} & k_{2}
\end{array}\right\} .
\end{aligned}
$$

Using the orthogonality relation for $\mathrm{C}-\mathrm{G}$ coefficients given in Eq. A.1 leads to

$$
\begin{aligned}
& \left\langle\Psi_{I, M_{f}}\left(\boldsymbol{r}_{1}, \boldsymbol{r}_{2}\right)\left|v_{p p}^{\sigma \sigma}\left(r_{p}\right) \boldsymbol{\sigma}_{0} \cdot \boldsymbol{\sigma}_{1}\right| \Psi_{I, M_{i}}\left(\boldsymbol{r}_{1}, \boldsymbol{r}_{2}\right)\right\rangle \\
& =(-)^{3 j_{1}-j_{1}^{\prime}} \sqrt{8 \pi} \hat{I} \hat{j}_{1} \hat{j}_{1}^{\prime} \hat{\ell}_{1}^{\prime} \int_{0}^{\infty} r_{1}^{2} d r_{1} u_{\ell_{1}^{\prime} j_{1}^{\prime}}\left(r_{1}\right) \sum_{K Q \mu_{0}} \frac{f_{K}^{(\sigma \sigma)}\left(R, r_{1}\right)}{\hat{K}^{2}} u_{\ell_{1} j_{1}}\left(r_{1}\right) Y_{K Q}(\hat{\boldsymbol{R}}) \\
& \quad \times \sigma_{1 \mu_{0}}^{(0)}(-)^{\mu_{0}+1}\left(\ell_{1}^{\prime} 0 K 0 \mid \ell_{1} 0\right) \sum_{k_{I} q_{I} k_{1} q_{1} k_{2} q_{2}} \hat{k}_{I}^{2} W\left(k_{I} j_{1} I j_{2} ; j_{1}^{\prime} I\right) \delta_{1, k_{1}} \delta_{\mu_{0}, q_{1}} \\
& \quad \times \delta_{k_{2}, K} \delta_{q_{2},-Q}\left(I M_{i} k_{I} q_{I} \mid I M_{f}\right)(-)^{k_{1}-k_{2}-q_{1}+q_{2}} \hat{k}_{1}\left(k_{1} q_{1} k_{I} q_{I} \mid k_{2} q_{2}\right)\left\{\begin{array}{ccc}
j_{1} & j_{1}^{\prime} & k_{I} \\
\frac{1}{2} & \frac{1}{2} & k_{1} \\
\ell_{1} & \ell_{1}^{\prime} & k_{2}
\end{array}\right\} .
\end{aligned}
$$


Finally, from Eq. C.5 it is easy to obtain the result from Section 3.5

$$
\begin{aligned}
\left\langle\Psi_{I, M_{f}}\left(\boldsymbol{r}_{1}, \boldsymbol{r}_{2}\right)\left|v_{p p}^{\sigma \sigma}\left(r_{p}\right) \boldsymbol{\sigma}_{0} \cdot \boldsymbol{\sigma}_{1}\right| \Psi_{I, M_{i}}\left(\boldsymbol{r}_{1}, \boldsymbol{r}_{2}\right)\right\rangle \\
=(-)^{3 j_{1}-j_{1}^{\prime}} \sqrt{24 \pi} \hat{I} \hat{j}_{1} \hat{j}_{1}^{\prime} \hat{\ell}_{1}^{\prime} \int_{0}^{\infty} r_{1}^{2} d r_{1} u_{\ell_{1}^{\prime} j_{1}^{\prime}}\left(r_{1}\right) \sum_{k_{I} k_{2}} \frac{f_{k_{2}}^{(\sigma \sigma)}\left(R, r_{1}\right)}{\hat{k}_{2}^{2}} u_{\ell_{1} j_{1}}\left(r_{1}\right) \\
\quad \times(-)^{k_{2}}\left(\ell_{1}^{\prime} 0 k_{2} 0 \mid \ell_{1} 0\right) \hat{k_{I}} W\left(k_{I} j_{1} I j_{2} ; j_{1}^{\prime} I\right)\left\{\begin{array}{ccc}
j_{1} & j_{1}^{\prime} & k_{I} \\
\frac{1}{2} & \frac{1}{2} & 1 \\
\ell_{1} & \ell_{1}^{\prime} & k_{2}
\end{array}\right\}\left\langle I, M_{f}\left|\boldsymbol{S}_{k_{I} k_{2}}\right| I, M_{i}\right\rangle,
\end{aligned}
$$

where $\left\langle I, M_{f}\left|S_{k_{I} k_{2}}\right| I, M_{i}\right\rangle$ is the general spin-spin operator given by Eq. 3.30.

\section{C.2 Folding of Tensor NN Interaction Term}

In this and the following sections, the details of the folding formalism summarised in Section 3.6 will be given for the tensor term $v_{p p}^{t r}\left(r_{p}\right) \boldsymbol{S}_{01}$ in the NN interaction.

\section{C.2.1 Form of the Tensor Potential}

The relationship between the tensor operators

$$
\left[3\left(\boldsymbol{\sigma}_{0} \cdot \hat{\boldsymbol{r}}_{p}\right)\left(\boldsymbol{\sigma}_{1} \cdot \hat{\boldsymbol{r}}_{p}\right)-\boldsymbol{\sigma}_{0} \cdot \boldsymbol{\sigma}_{1}\right]=2 \sqrt{\frac{8 \pi}{5}} \sum_{\mu}(-)^{\mu} \tau_{2-\mu}(\boldsymbol{S}) Y_{2 \mu}\left(\hat{\boldsymbol{r}}_{p}\right)
$$

discussed in Section 3.6, can be derived using

$$
\tau_{2-\mu}(\boldsymbol{S})=\frac{\sqrt{3}}{2} \sum_{\mu_{1} \mu_{2}}\left(1 \mu_{1} 1 \mu_{2} \mid 2-\mu\right) \tau_{1 \mu_{1}}\left(\boldsymbol{\sigma}_{0}\right) \tau_{1 \mu_{2}}\left(\boldsymbol{\sigma}_{1}\right)
$$

which separates the rank-2 spin operator for the total spin $\tau_{2-\mu}(\boldsymbol{S})$, into rank-1 spin operators of the component spins $\tau_{1 \mu_{1}}\left(\boldsymbol{\sigma}_{0}\right)$ and $\tau_{1 \mu_{2}}\left(\boldsymbol{\sigma}_{1}\right)$ where $\boldsymbol{S}=\frac{1}{2}\left(\boldsymbol{\sigma}_{0}+\boldsymbol{\sigma}_{1}\right)$. The coefficient $\sqrt{3} / 2$ is obtained by comparing the matrix elements for the three spin operators. Also required to derive the relationship in Eq. C.7 is

$$
Y_{1 \mu_{1}}\left(\hat{\boldsymbol{r}}_{p}\right) Y_{1 \mu_{2}}\left(\hat{\boldsymbol{r}}_{p}\right)=(-)^{\mu_{1}} \frac{\delta_{\mu_{1},-\mu_{2}}}{4 \pi}+\sqrt{\frac{6}{5(4 \pi)}} \sum_{\mu} Y_{2 \mu}\left(\hat{\boldsymbol{r}}_{p}\right)\left(1 \mu_{1} 1 \mu_{2} \mid 2 \mu\right)
$$


where Eq. C.9 is a specific case of Eq. A.16. Substituting these relationships into the right hand side of Eq. C.7 leads to

$$
4 \pi \sum_{\mu_{1} \mu_{2}}(-)^{\mu_{1}+\mu_{2}} \tau_{1 \mu_{1}}\left(\boldsymbol{\sigma}_{0}\right) \tau_{1 \mu_{2}}\left(\boldsymbol{\sigma}_{1}\right)\left[Y_{1-\mu_{1}}\left(\hat{\boldsymbol{r}}_{p}\right) Y_{1-\mu_{2}}\left(\hat{\boldsymbol{r}}_{p}\right)-(-)^{\mu_{1}} \frac{\delta_{\mu_{1},-\mu_{2}}}{4 \pi}\right] .
$$

Now, using $Y_{1-\mu_{1}}\left(\hat{\boldsymbol{r}}_{p}\right)=\sqrt{3 / 4 \pi}\left(\hat{\boldsymbol{r}}_{p}\right)_{1-\mu_{1}}[96]$, where $\mu_{1}=-1,0,1$, (the spherical components of vector $\boldsymbol{r}_{p}$ ) it is easy to show

$$
\begin{aligned}
3 \sum_{\mu_{1}}(-)^{\mu_{1}} \tau_{1 \mu_{1}}\left(\boldsymbol{\sigma}_{0}\right)\left(\hat{\boldsymbol{r}}_{p}\right)_{1-\mu_{1}} \sum_{\mu_{2}}(-)^{\mu_{2}} \tau_{1 \mu_{2}}\left(\boldsymbol{\sigma}_{1}\right)\left(\hat{\boldsymbol{r}}_{p}\right)_{1-\mu_{2}}-\sum_{\mu_{1}}(-)^{\mu_{1}} \tau_{1 \mu_{1}}\left(\boldsymbol{\sigma}_{0}\right) \tau_{1-\mu_{1}}\left(\boldsymbol{\sigma}_{1}\right) \\
=\left[3\left(\boldsymbol{\sigma}_{0} \cdot \hat{\boldsymbol{r}}_{p}\right)\left(\boldsymbol{\sigma}_{1} \cdot \hat{\boldsymbol{r}}_{p}\right)-\boldsymbol{\sigma}_{0} \cdot \boldsymbol{\sigma}_{1}\right]
\end{aligned}
$$

\section{C.3 Folding of Tensor Term 1}

Continuing the discussion in Section 3.6.2, taking only the first tensor term $R^{2} Y_{2 \mu}(\hat{\boldsymbol{R}})$ in Eq. 3.38 yields

\section{Term 1}

$$
\begin{aligned}
& =(-)^{3 j_{1}-j_{1}^{\prime}} \hat{I} \hat{j}_{1} \hat{j}_{1}^{\prime} \int d \boldsymbol{r}_{1} \sum_{\lambda_{1}^{\prime} \lambda_{1} m_{s 1}^{\prime} m_{s 1}} Y_{\ell_{1}^{\prime} \lambda_{1}^{\prime}}^{*}\left(\hat{\boldsymbol{r}}_{1}\right) \chi_{\frac{1}{2} m_{s 1}^{\prime}}^{*} u_{\ell_{1}^{\prime} j_{1}^{\prime}}\left(r_{1}\right) 4 \pi \sum_{K Q} \frac{f_{K}^{(t r)}\left(R, r_{1}\right)}{\hat{K}^{2}} Y_{K Q}(\hat{\boldsymbol{R}}) \\
& \times Y_{K Q}^{*}\left(\hat{\boldsymbol{r}}_{1}\right) 2 \sqrt{\frac{8 \pi}{5}} \sum_{\mu \mu_{0} \mu_{1}}(-)^{\mu} \frac{\sqrt{3}}{2}\left(1 \mu_{0} 1 \mu_{1} \mid 2-\mu\right) \tau_{1 \mu_{0}}\left(\boldsymbol{\sigma}_{0}\right) \tau_{1 \mu_{1}}\left(\boldsymbol{\sigma}_{1}\right) \frac{1}{r_{p}^{2}} R^{2} Y_{2 \mu}(\hat{\boldsymbol{R}}) Y_{\ell_{1} \lambda_{1}}\left(\hat{\boldsymbol{r}}_{1}\right) \\
& \times \quad \chi_{\frac{1}{2} m_{s 1}} u_{\ell_{1} j_{1}}\left(r_{1}\right)(-)^{1 / 2+m_{s 1}^{\prime}-\ell_{1}^{\prime}-\lambda_{1}} \sum_{k_{I} q_{I}} \hat{k}_{I}^{2} W\left(k_{I} j_{1} I j_{2} ; j_{1}^{\prime} I\right)\left(I M_{i} k_{I} q_{I} \mid I M_{f}\right) \sum_{k_{1} q_{1} k_{2} q_{2}}(-)^{k_{1}-k_{2}} \hat{k}_{1} \\
& \times\left(\frac{1}{2} m_{s 1} \frac{1}{2}-m_{s 1}^{\prime} \mid k_{1} q_{1}\right)\left(\ell_{1}-\lambda_{1} \ell_{1}^{\prime} \lambda_{1}^{\prime} \mid k_{2} q_{2}\right)\left(k_{1} q_{1} k_{I} q_{I} \mid k_{2} q_{2}\right)\left\{\begin{array}{ccc}
j_{1} & j_{1}^{\prime} & k_{I} \\
\frac{1}{2} & \frac{1}{2} & k_{1} \\
\ell_{1} & \ell_{1}^{\prime} & k_{2}
\end{array}\right\} .
\end{aligned}
$$

Performing the angular integration of the three spherical harmonics dependant on $\hat{\boldsymbol{r}}_{1}$ using Eq. C.2, coupling the two spherical harmonics dependant on $\hat{\boldsymbol{R}}$ using Eq. A.16 and substituting in for the matrix element $\left\langle\frac{1}{2} \sigma_{1}^{\prime}\left|\sigma_{1 \mu_{1}}^{(1)}\right| \frac{1}{2} \sigma_{1}\right\rangle$ using Eq. C.3 leads to 
Term 1

$$
\begin{aligned}
& =(-)^{3 j_{1}-j_{1}^{\prime}} 2 \sqrt{12 \pi} \hat{I} \hat{j}_{1} \hat{j}_{1}^{\prime} \hat{\ell}_{1}^{\prime} R^{2} \int_{0}^{\infty} r_{1}^{2} d r_{1} u_{\ell_{1}^{\prime} j_{1}^{\prime}}\left(r_{1}\right) \sum_{K Q} \frac{f_{K}^{(t r)}\left(R, r_{1}\right)}{r_{p}^{2} \hat{K}} u_{\ell_{1} j_{1}}\left(r_{1}\right) \\
& \times \sum_{\mu \mu_{0} \mu_{1}}(-)^{\mu+1}\left(1 \mu_{0} 1 \mu_{1} \mid 2-\mu\right) \tau_{1 \mu_{0}}\left(\sigma_{0}\right) \sum_{C \Gamma} \frac{Y_{C \Gamma}(\hat{\boldsymbol{R}})}{\hat{C}}(K Q 2 \mu \mid C \Gamma)(K 020 \mid C 0) \\
& \times \sum_{\lambda_{1}^{\prime} \lambda_{1} m_{s 1}^{\prime} m_{s 1}}\left(\frac{1}{2} m_{s 1} \frac{1}{2}-m_{s 1}^{\prime} \mid 1-\mu_{1}\right)\left(\ell_{1}-\lambda_{1} \ell_{1}^{\prime} \lambda_{1}^{\prime} \mid K-Q\right)\left(\ell_{1}^{\prime} 0 K 0 \mid \ell_{1} 0\right) \\
& \times \sum_{k_{I} q_{I}}{\hat{k_{I}}}^{2} W\left(k_{I} j_{1} I j_{2} ; j_{1}^{\prime} I\right)\left(I M_{i} k_{I} q_{I} \mid I M_{f}\right) \sum_{k_{1} q_{1} k_{2} q_{2}}(-)^{k_{1}-k_{2}-q_{1}+q_{2}} \hat{k}_{1} \\
& \times \quad\left(\frac{1}{2} m_{s 1} \frac{1}{2}-m_{s 1}^{\prime} \mid k_{1} q_{1}\right)\left(\ell_{1}-\lambda_{1} \ell_{1}^{\prime} \lambda_{1}^{\prime} \mid k_{2} q_{2}\right)\left(k_{1} q_{1} k_{I} q_{I} \mid k_{2} q_{2}\right)\left\{\begin{array}{ccc}
j_{1} & j_{1}^{\prime} & k_{I} \\
\frac{1}{2} & \frac{1}{2} & k_{1} \\
\ell_{1} & \ell_{1}^{\prime} & k_{2}
\end{array}\right\} .
\end{aligned}
$$

Using the orthogonality relation for $\mathrm{C}-\mathrm{G}$ coefficients given in Eq. A.1 results in

\section{Term 1}

$$
\begin{aligned}
& =(-)^{3 j_{1}-j_{1}^{\prime}} 6 \sqrt{4 \pi} \hat{I} \hat{j}_{1} \hat{j}_{1}^{\prime} \hat{\ell}_{1}^{\prime} R^{2} \int_{0}^{\infty} r_{1}^{2} d r_{1} u_{\ell_{1}^{\prime} j_{1}^{\prime}}\left(r_{1}\right) \sum_{k_{2} q_{2}} \frac{f_{k_{2}}^{(t r)}\left(R, r_{1}\right)}{r_{p}^{2} \hat{k}_{2}} u_{\ell_{1} j_{1}}\left(r_{1}\right) \\
& \times \sum_{\mu \mu_{0} \mu_{1}}(-)^{\mu+\mu_{1}-k_{2}+q_{2}}\left(1 \mu_{0} 1 \mu_{1} \mid 2-\mu\right) \tau_{1 \mu_{0}}\left(\boldsymbol{\sigma}_{0}\right) \sum_{C \Gamma} \frac{Y_{C \Gamma}(\hat{\boldsymbol{R}})}{\hat{C}}\left(k_{2}-q_{2} 2 \mu \mid C \Gamma\right)\left(k_{2} 020 \mid C 0\right) \\
& \times \quad\left(\ell_{1}^{\prime} 0 k_{2} 0 \mid \ell_{1} 0\right) \sum_{k_{I} q_{I}}{\hat{k_{I}}}^{2} W\left(k_{I} j_{1} I j_{2} ; j_{1}^{\prime} I\right)\left(I M_{i} k_{I} q_{I} \mid I M_{f}\right)\left(1-\mu_{1} k_{I} q_{I} \mid k_{2} q_{2}\right)\left\{\begin{array}{ccc}
j_{1} & j_{1}^{\prime} & k_{I} \\
\frac{1}{2} & \frac{1}{2} & 1 \\
\ell_{1} & \ell_{1}^{\prime} & k_{2}
\end{array}\right\} .
\end{aligned}
$$

The three C-G coefficients shown in red can be reduced using Eq. A.8 to give the final folded potential given in $\mathrm{Eq} 3.39$

\section{Term 1}

$$
\begin{aligned}
& =(-)^{3 j_{1}-j_{1}^{\prime}} 6 \sqrt{20 \pi} \hat{I} \hat{j}_{1} \hat{j}_{1}^{\prime} \hat{\ell}_{1}^{\prime} R^{2} \int_{0}^{\infty} r_{1}^{2} d r_{1} u_{\ell_{1}^{\prime} j_{1}^{\prime}}\left(r_{1}\right) \sum_{k_{I} k_{2} C} \frac{f_{k_{2}}^{(t r)}\left(R, r_{1}\right)}{r_{p}^{2} \hat{C}} u_{\ell_{1} j_{1}}\left(r_{1}\right)(-)^{-k_{I}} \\
& \times\left(\ell_{1}^{\prime} 0 k_{2} 0 \mid \ell_{1} 0\right)\left(k_{2} 020 \mid C 0\right) W\left(11 C k_{2} ; 2 k_{I}\right) \hat{k_{I}} W\left(k_{I} j_{1} I j_{2} ; j_{1}^{\prime} I\right) \\
& \times\left(\begin{array}{ccc}
j_{1} & j_{1}^{\prime} & k_{I} \\
\frac{1}{2} & \frac{1}{2} & 1 \\
\ell_{1} & \ell_{1}^{\prime} & k_{2}
\end{array}\right\}\left\langle I, M_{f}\left|S_{k_{I} C}\right| I, M_{i}\right\rangle .
\end{aligned}
$$




\section{C.4 Folding of Tensor Term 2}

The folded potential derived in Section 3.6.2, comes from taking only the second tensor term $r_{1}^{2} Y_{2 \mu}\left(\hat{\boldsymbol{r}}_{1}\right)$ in Eq. 3.38

\section{Term 2}

$=(-)^{3 j_{1}-j_{1}^{\prime}} \hat{I} \hat{j}_{1} \hat{j}_{1}^{\prime} \int d \boldsymbol{r}_{1} \sum_{\lambda_{1}^{\prime} \lambda_{1} m_{s 1}^{\prime} m_{s 1}} Y_{\ell_{1}^{\prime} \lambda_{1}^{\prime}}^{*}\left(\hat{\boldsymbol{r}}_{1}\right) \chi_{\frac{1}{2} m_{s 1}^{\prime}}^{*} u_{\ell_{1}^{\prime} j_{1}^{\prime}}\left(r_{1}\right) 4 \pi \sum_{K Q} \frac{f_{K}^{(t r)}\left(R, r_{1}\right)}{\hat{K}^{2}} Y_{K Q}(\hat{\boldsymbol{R}})$

$\times Y_{K Q}^{*}\left(\hat{\boldsymbol{r}}_{1}\right) 2 \sqrt{\frac{8 \pi}{5}} \sum_{\mu \mu_{0} \mu_{1}}(-)^{\mu} \frac{\sqrt{3}}{2}\left(1 \mu_{0} 1 \mu_{1} \mid 2-\mu\right) \tau_{1 \mu_{0}}\left(\boldsymbol{\sigma}_{0}\right) \tau_{1 \mu_{1}}\left(\boldsymbol{\sigma}_{1}\right) \frac{1}{r_{p}^{2}} r_{1}^{2} Y_{2 \mu}\left(\hat{\boldsymbol{r}}_{1}\right) Y_{\ell_{1} \lambda_{1}}\left(\hat{\boldsymbol{r}}_{1}\right)$

$\times \chi_{\frac{1}{2} m_{s 1}} u_{\ell_{1} j_{1}}\left(r_{1}\right)(-)^{1 / 2+m_{s 1}^{\prime}-\ell_{1}^{\prime}-\lambda_{1}} \sum_{k_{I} q_{I}} \hat{k}_{I}^{2} W\left(k_{I} j_{1} I j_{2} ; j_{1}^{\prime} I\right)\left(I M_{i} k_{I} q_{I} \mid I M_{f}\right) \sum_{k_{1} q_{1} k_{2} q_{2}}(-)^{k_{1}-k_{2}} \hat{k}_{1}$

$\times\left(\frac{1}{2} m_{s 1} \frac{1}{2}-m_{s 1}^{\prime} \mid k_{1} q_{1}\right)\left(\ell_{1}-\lambda_{1} \ell_{1}^{\prime} \lambda_{1}^{\prime} \mid k_{2} q_{2}\right)\left(k_{1} q_{1} k_{I} q_{I} \mid k_{2} q_{2}\right)\left\{\begin{array}{ccc}j_{1} & j_{1}^{\prime} & k_{I} \\ \frac{1}{2} & \frac{1}{2} & k_{1} \\ \ell_{1} & \ell_{1}^{\prime} & k_{2}\end{array}\right\}$.

Coupling the four spherical harmonics dependant on $\hat{\boldsymbol{r}}_{1}$ using Eq. A.16 twice and performing the angular integration of the two resultant spherical harmonics using Eq. A.15 gives

$$
\begin{aligned}
\int d \Omega_{r_{1}} Y_{\ell_{1}^{\prime} \lambda_{1}^{\prime}}^{*}\left(\hat{\boldsymbol{r}}_{1}\right) Y_{K Q}^{*}\left(\hat{\boldsymbol{r}}_{1}\right) Y_{2 \mu}\left(\hat{\boldsymbol{r}}_{1}\right) Y_{\ell_{1} \lambda_{1}}\left(\hat{\boldsymbol{r}}_{1}\right) & =\sum_{C \Gamma} \frac{\hat{\ell}_{1}^{\prime} \hat{K} \sqrt{5} \hat{\ell}_{1}}{4 \pi \hat{C}^{2}}\left(\ell_{1}^{\prime} \lambda_{1}^{\prime} K Q \mid C \Gamma\right)\left(\ell_{1}^{\prime} 0 K 0 \mid C 0\right) \\
& \times\left(2 \mu \ell_{1} \lambda_{1} \mid C \Gamma\right)\left(20 \ell_{1} 0 \mid C 0\right),
\end{aligned}
$$

inserting this into Eq. C.16 and using Eq. C.3 for the matrix element $\left\langle\frac{1}{2} m_{s 1}^{\prime}\left|\sigma_{1 \mu_{1}}^{(1)}\right| \frac{1}{2} m_{s 1}\right\rangle$ gives

\section{Term 2}

$$
\begin{aligned}
& =(-)^{3 j_{1}-j_{1}^{\prime}-\ell_{1}^{\prime}} 2 \sqrt{12 \pi} \hat{I} \hat{j}_{1} \hat{j}_{1}^{\prime} \hat{\ell}_{1} \hat{\ell}_{1}^{\prime} \int_{0}^{\infty} r_{1}^{4} d r_{1} u_{\ell_{1}^{\prime} j_{1}^{\prime}}\left(r_{1}\right) \sum_{K Q} \frac{f_{K}^{(t r)}\left(R, r_{1}\right)}{r_{p}^{2} \hat{K}} u_{\ell_{1} j_{1}}\left(r_{1}\right) \\
& \times Y_{K Q}(\hat{\boldsymbol{R}}) \sum_{\substack{\mu \mu_{0} \mu_{1} \\
\lambda_{1}^{\prime} \lambda_{1} m_{s 1}^{\prime} m_{s 1}}}(-)^{\mu+1}\left(1 \mu_{0} 1 \mu_{1} \mid 2-\mu\right) \tau_{1 \mu_{0}}\left(\sigma_{0}\right)\left(\frac{1}{2} m_{s 1} \frac{1}{2}-m_{s 1}^{\prime} \mid 1-\mu_{1}\right) \\
& \times \sum_{C \Gamma} \frac{1}{\hat{C}^{2}}\left(\ell_{1}^{\prime} \lambda_{1}^{\prime} K Q \mid C \Gamma\right)\left(\ell_{1}^{\prime} 0 K 0 \mid C 0\right)\left(2 \mu \ell_{1} \lambda_{1} \mid C \Gamma\right)\left(20 \ell_{1} 0 \mid C 0\right) \\
& \times \sum_{k_{I} q_{I}} \hat{k}_{I}^{2} W\left(k_{I} j_{1} I j_{2} ; j_{1}^{\prime} I\right)\left(I M_{i} k_{I} q_{I} \mid I M_{f}\right) \sum_{k_{1} q_{1} k_{2} q_{2}}(-)^{k_{1}-k_{2}-q_{1}-\lambda_{1} \hat{k}_{1}} \\
& \times\left(\frac{1}{2} m_{s 1} \frac{1}{2}-m_{s 1}^{\prime} \mid k_{1} q_{1}\right)\left(\ell_{1}-\lambda_{1} \ell_{1}^{\prime} \lambda_{1}^{\prime} \mid k_{2} q_{2}\right)\left(k_{1} q_{1} k_{I} q_{I} \mid k_{2} q_{2}\right)\left\{\begin{array}{ccc}
j_{1} & j_{1}^{\prime} & k_{I} \\
\frac{1}{2} & \frac{1}{2} & k_{1} \\
\ell_{1} & \ell_{1}^{\prime} & k_{2}
\end{array}\right\} .
\end{aligned}
$$


Using the orthogonality of the C-G coefficients (Eq. A.1) simplifies this to

Term 2

$$
\begin{aligned}
& =(-)^{3 j_{1}-j_{1}^{\prime}-\ell_{1}^{\prime}} 6 \sqrt{4 \pi} \hat{I} \hat{j}_{1} \hat{j}_{1}^{\prime} \hat{\ell}_{1} \hat{\ell}_{1}^{\prime} \int_{0}^{\infty} r_{1}^{4} d r_{1} u_{\ell_{1}^{\prime} j_{1}^{\prime}}\left(r_{1}\right) \sum_{K Q} \frac{f_{K}^{(t r)}\left(R, r_{1}\right)}{r_{p}^{2} \hat{K}} u_{\ell_{1} j_{1}}\left(r_{1}\right) \\
& \times Y_{K Q}(\hat{\boldsymbol{R}}) \sum_{\substack{\mu \mu_{0} \mu_{1} \\
\lambda_{1}^{\prime} \lambda_{1}}}(-)^{\mu+\mu_{1}}\left(1 \mu_{0} 1 \mu_{1} \mid 2-\mu\right) \tau_{1 \mu_{0}}\left(\boldsymbol{\sigma}_{0}\right) \\
& \times \sum_{C \Gamma} \frac{1}{\hat{C}^{2}}\left(\ell_{1}^{\prime} \lambda_{1}^{\prime} K Q \mid C \Gamma\right)\left(\ell_{1}^{\prime} 0 K 0 \mid C 0\right)\left(2 \mu \ell_{1} \lambda_{1} \mid C \Gamma\right)\left(20 \ell_{1} 0 \mid C 0\right) \\
& \times \sum_{k_{I} q_{I}} \hat{k}_{I}^{2} W\left(k_{I} j_{1} I j_{2} ; j_{1}^{\prime} I\right)\left(I M_{i} k_{I} q_{I} \mid I M_{f}\right) \sum_{k_{2} q_{2}}(-)^{-k_{2}-\lambda_{1}} \\
& \times\left(\ell_{1}-\lambda_{1} \ell_{1}^{\prime} \lambda_{1}^{\prime} \mid k_{2} q_{2}\right)\left(1-\mu_{1} k_{I} q_{I} \mid k_{2} q_{2}\right)\left\{\begin{array}{ccc}
j_{1} & j_{1}^{\prime} & k_{I} \\
\frac{1}{2} & \frac{1}{2} & 1 \\
\ell_{1} & \ell_{1}^{\prime} & k_{2}
\end{array}\right\} .
\end{aligned}
$$

The graphical representation of the five C-G coefficients in Eq. C.19 to be coupled is shown in Figure C.1. The three C-G coefficients circled in Figure C.1 can be reduced using the relationship between Racah coefficients and $\mathrm{C}-\mathrm{G}$ coefficients given in Eq. A.8, this leads to

\section{Term 2}

$$
\begin{aligned}
& =(-)^{3 j_{1}-j_{1}^{\prime}-\ell_{1}^{\prime}+\ell_{1}} 6 \sqrt{4 \pi} \hat{I} \hat{j}_{1} \hat{j}_{1}^{\prime} \hat{\ell}_{1} \hat{\ell}_{1}^{\prime} \int_{0}^{\infty} r_{1}^{4} d r_{1} u_{\ell_{1}^{\prime} j_{1}^{\prime}}\left(r_{1}\right) \sum_{K Q} \frac{f_{K}^{(t r)}\left(R, r_{1}\right)}{r_{p}^{2} \hat{K}^{2}} u_{\ell_{1} j_{1}}\left(r_{1}\right) \\
& \times Y_{K Q}(\hat{\boldsymbol{R}}) \sum_{\mu \mu_{0} \mu_{1}}(-)^{\mu+\mu_{1}}\left(1 \mu_{0} 1 \mu_{1} \mid 2-\mu\right) \tau_{1 \mu_{0}}\left(\boldsymbol{\sigma}_{0}\right) \sum_{C \Gamma}\left(\ell_{1}^{\prime} 0 K 0 \mid C 0\right)\left(20 \ell_{1} 0 \mid C 0\right) \\
& \times \sum_{k_{I} q_{I}} \hat{k}_{I}^{2} W\left(k_{I} j_{1} I j_{2} ; j_{1}^{\prime} I\right)\left(I M_{i} k_{I} q_{I} \mid I M_{f}\right) \sum_{k_{2} q_{2}}(-)^{q_{2}} \hat{k}_{2}\left(2 \mu k_{2}-q_{2} \mid K Q\right) \\
& \times W\left(2 \ell_{1} K \ell_{1}^{\prime} ; C k_{2}\right)\left(1-\mu_{1} k_{I} q_{I} \mid k_{2} q_{2}\right)\left\{\begin{array}{ccc}
j_{1} & j_{1}^{\prime} & k_{I} \\
\frac{1}{2} & \frac{1}{2} & 1 \\
\ell_{1} & \ell_{1}^{\prime} & k_{2}
\end{array}\right\} .
\end{aligned}
$$

The remaining three C-G coefficients shown in red can also be reduced using Eq. A.8 to give the final folded potential of Eq. 3.44 


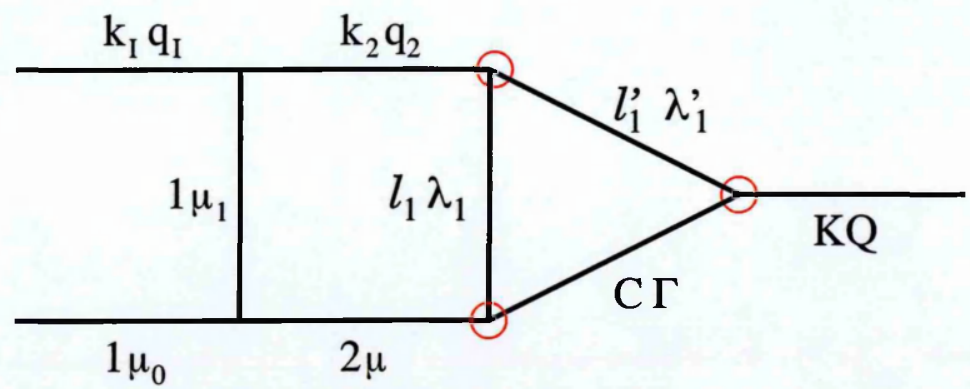

Figure C.1: The graphical representation of the C-G coefficients in Eq. C.19.

Term 2

$$
\begin{aligned}
& =(-)^{3 j_{1}-j_{1}^{\prime}-\ell_{1}^{\prime}+\ell_{1}} 6 \sqrt{20 \pi} \hat{I} \hat{j}_{1} \hat{j}_{1}^{\prime} \hat{\ell}_{1} \hat{\ell}_{1}^{\prime} \int_{0}^{\infty} r_{1}^{4} d r_{1} u_{\ell_{1}^{\prime} j_{1}^{\prime}}\left(r_{1}\right) \sum_{k_{I} k_{2} C K} \frac{f_{K}^{(t r)}\left(R, r_{1}\right)}{r_{p}^{2} \hat{K}^{2}} \\
& \times u_{\ell_{1} j_{1}}\left(r_{1}\right)(-)^{K-k_{I}} \hat{k}_{2}^{2}\left(\ell_{1}^{\prime} 0 K 0 \mid C 0\right)\left(20 \ell_{1} 0 \mid C 0\right) W\left(11 K k_{2} ; 2 k_{I}\right) \\
& \times W\left(2 \ell_{1} K \ell_{1}^{\prime} ; C k_{2}\right) \hat{k}_{I} W\left(k_{I} j_{1} I j_{2} ; j_{1}^{\prime} I\right)\left\{\begin{array}{ccc}
j_{1} & j_{1}^{\prime} & k_{I} \\
\frac{1}{2} & \frac{1}{2} & 1 \\
\ell_{1} & \ell_{1}^{\prime} & k_{2}
\end{array}\right\}\left\langle I, M_{f}\left|S_{k_{I} K}\right| I, M_{i}\right\rangle .
\end{aligned}
$$

\section{C.5 Folding of Tensor Term 3}

Following on from the discussion in Section 3.6.4, taking only the third tensor term $\sqrt{4 \pi} \sqrt{\frac{10}{3}} \sum_{\alpha \beta}(1 \alpha 1 \beta \mid 2 \mu) R Y_{1 \alpha}(\hat{\boldsymbol{R}}) r_{1} Y_{1 \beta}\left(\hat{r}_{1}\right)$ in Eq. 3.38 yields

\section{Term 3}

$$
\begin{aligned}
& =(-)^{3 j_{1}-j_{1}^{\prime}} \hat{I} \hat{j}_{1} \hat{j}_{1}^{\prime} \int d \boldsymbol{r}_{1} \sum_{\lambda_{1}^{\prime} \lambda_{1} m_{s 1}^{\prime} m_{s 1}} Y_{\ell_{1}^{\prime} \lambda_{1}^{\prime}}^{*}\left(\hat{\boldsymbol{r}}_{1}\right) \chi_{\frac{1}{2} m_{s 1}^{\prime}}^{*} u_{\ell_{1}^{\prime} j_{1}^{\prime}}\left(r_{1}\right) 4 \pi \sum_{K Q} \frac{f_{K}^{(t r)}\left(R, r_{1}\right)}{\hat{K}^{2}} \\
& \times Y_{K Q}(\hat{\boldsymbol{R}}) Y_{K Q}^{*}\left(\hat{\boldsymbol{r}}_{1}\right) 2 \sqrt{\frac{8 \pi}{5}} \sum_{\mu \mu_{0} \mu_{1}}(-)^{\mu} \frac{\sqrt{3}}{2}\left(1 \mu_{0} 1 \mu_{1} \mid 2-\mu\right) \tau_{1 \mu_{0}}\left(\boldsymbol{\sigma}_{0}\right) \tau_{1 \mu_{1}}\left(\boldsymbol{\sigma}_{1}\right) \frac{1}{r_{p}^{2}} \\
& \times\left\{-\sqrt{4 \pi} \sqrt{\frac{10}{3}} \sum_{\alpha \beta}(1 \alpha 1 \beta 2 \mu) R Y_{1 \alpha}(\hat{\boldsymbol{R}})_{r_{1}} Y_{1 \beta}\left(\hat{r}_{1}\right)\right\} Y_{\ell_{1} \lambda_{1}}\left(\hat{\boldsymbol{r}}_{1}\right) \chi_{\frac{1}{2} m_{s 1}} u_{\ell_{1} j_{1}}\left(r_{1}\right) \\
& \times \quad(-)^{1 / 2+m_{s 1}^{\prime}-\ell_{1}^{\prime}-\lambda_{1}} \sum_{k_{I} q_{I}}{\hat{k_{I}}}^{2} W\left(k_{I} j_{1} I j_{2} ; j_{1}^{\prime} I\right)\left(I M_{i} k_{I} q_{I} \mid I M_{f}\right) \sum_{k_{1} q_{1} k_{2} q_{2}}(-)^{k_{1}-k_{2}} \hat{k}_{1} \\
& \times\left(\frac{1}{2} m_{s 1} \frac{1}{2}-m_{s 1}^{\prime} \mid k_{1} q_{1}\right)\left(\ell_{1}-\lambda_{1} \ell_{1}^{\prime} \lambda_{1}^{\prime} \mid k_{2} q_{2}\right)\left(k_{1} q_{1} k_{I} q_{I} \mid k_{2} q_{2}\right)\left\{\begin{array}{ccc}
j_{1} & j_{1}^{\prime} & k_{I} \\
\frac{1}{2} & \frac{1}{2} & k_{1} \\
\ell_{1} & \ell_{1}^{\prime} & k_{2}
\end{array}\right\} .
\end{aligned}
$$


Coupling the two spherical harmonics dependent on $\hat{\boldsymbol{R}}$ using Eq. A.16 and coupling the four spherical harmonics dependant on $\hat{\boldsymbol{r}}_{1}$ and performing the angular integration of the two resultant spherical harmonics using Eq. C.17, leads to

\section{Term 3}

$$
\begin{aligned}
& =(-)^{3 j_{1}-j_{1}^{\prime}+1-\ell_{1}^{\prime}} 6 \sqrt{4 \pi} \hat{I} \hat{j}_{1} \hat{j}_{1}^{\prime} \hat{\ell}_{1} \hat{\ell}_{1}^{\prime} R \int_{0}^{\infty} r_{1}^{3} d r_{1} u_{\ell_{1}^{\prime} j_{1}^{\prime}}\left(r_{1}\right) \sum_{K Q} \frac{f_{K}^{(t r)}\left(R, r_{1}\right)}{r_{p}^{2}} u_{\ell_{1} j_{1}}\left(r_{1}\right) \\
& \times \quad \sum_{\substack{\mu \mu_{0} \mu_{1} \\
\lambda_{1}^{\prime} \lambda_{1} m_{s 1}^{\prime} m_{s 1}}}(-)^{\mu+1 / 2+m_{s 1}^{\prime}-\lambda_{1}}\left(1 \mu_{0} 1 \mu_{1} \mid 2-\mu\right) \tau_{1 \mu_{0}}\left(\boldsymbol{\sigma}_{0}\right)\left\langle\frac{1}{2} m_{s 1}^{\prime}\left|\sigma_{1 \mu_{1}}^{(1)}\right| \frac{1}{2} m_{s 1}\right\rangle \sum_{\alpha \beta}(1 \alpha 1 \beta \mid 2 \mu) \\
& \times \quad \sum_{C \Gamma C^{\prime} \Gamma^{\prime}} \frac{1}{\hat{C}^{2}}\left(\ell_{1}^{\prime} \lambda_{1}^{\prime} K Q \mid C \Gamma\right)\left(\ell_{1}^{\prime} 0 K 0 \mid C 0\right)\left(1 \beta \ell_{1} \lambda_{1} \mid C \Gamma\right)\left(10 \ell_{1} 0 \mid C 0\right) \frac{Y_{C^{\prime} \Gamma^{\prime}}(\hat{\boldsymbol{R}})}{\hat{C}^{\prime}} \\
& \times \quad\left(K Q 1 \alpha \mid C^{\prime \prime} \Gamma^{\prime \prime}\right)\left(K 010 \mid C^{\prime} 0\right) \sum_{k_{I} q_{I}}{\hat{k_{I}}}^{2} W\left(k_{I} j_{1} I j_{2} ; j_{1}^{\prime} I\right)\left(I M_{i} k_{I} q_{I} \mid I M_{f}\right) \sum_{k_{1} q_{1} k_{2} q_{2}}(-)^{k_{1}-k_{2}} \\
& \times \quad \hat{k}_{1}\left(\frac{1}{2} m_{s 1} \frac{1}{2}-m_{s 1}^{\prime} \mid k_{1} q_{1}\right)\left(\ell_{1}-\lambda_{1} \ell_{1}^{\prime} \lambda_{1}^{\prime} \mid k_{2} q_{2}\right)\left(k_{1} q_{1} k_{I} q_{I} \mid k_{2} q_{2}\right)\left\{\begin{array}{ccc}
j_{1} & j_{1}^{\prime} & k_{I} \\
\frac{1}{2} & \frac{1}{2} & k_{1} \\
\ell_{1} & \ell_{1}^{\prime} & k_{2}
\end{array}\right\} .
\end{aligned}
$$

Using Eq. C.3 for the matrix element $\left\langle\frac{1}{2} m_{s 1}^{\prime}\left|\sigma_{1 \mu_{1}}^{(1)}\right| \frac{1}{2} m_{s 1}\right\rangle$ and using the orthogonality of the C-G coefficients from Eq. A.1 in the same way as the previous two direct tensor terms results in

\section{Term 3}

$$
\begin{aligned}
& =(-)^{3 j_{1}-j_{1}^{\prime}+1-\ell_{1}^{\prime}} 6 \sqrt{6} \sqrt{4 \pi} \hat{I} \hat{j}_{1} \hat{j}_{1}^{\prime} \hat{\ell}_{1} \hat{\ell}_{1}^{\prime} R \int_{0}^{\infty} r_{1}^{3} d r_{1} u_{\ell_{1}^{\prime} j_{1}^{\prime}}\left(r_{1}\right) \sum_{K Q} \frac{f_{K}^{(t r)}\left(R, r_{1}\right)}{r_{p}^{2}} u_{\ell_{1} j_{1}}\left(r_{1}\right) \\
& \times \sum_{\substack{\mu \mu_{0} \mu_{1} \\
\lambda_{1}^{\prime} \lambda_{1} \alpha \beta}}(-)^{\mu+\mu_{1}-\lambda_{1}}\left(1 \mu_{0} 1 \mu_{1} \mid 2-\mu\right) \tau_{1 \mu_{0}}\left(\boldsymbol{\sigma}_{0}\right)(1 \alpha 1 \beta \mid 2 \mu) \sum_{C \Gamma C^{\prime} \Gamma^{\prime}}\left(\ell_{1}^{\prime} \lambda_{1}^{\prime} K Q \mid C \Gamma\right) \\
& \times \quad\left(\ell_{1}^{\prime} 0 K 0 \mid C 0\right)\left(1 \beta \ell_{1} \lambda_{1} \mid C \Gamma\right)\left(10 \ell_{1} 0 \mid C 0\right) \frac{Y_{C^{\prime} \Gamma^{\prime}}(\hat{\boldsymbol{R}})}{\hat{C}^{\prime} \hat{C}^{2}}\left(K Q 1 \alpha \mid C^{\prime} \Gamma^{\prime}\right)\left(K 010 \mid C^{\prime} 0\right) \\
& \times \sum_{k_{I} q_{I}} \hat{k}_{I}^{2} W\left(k_{I} j_{1} I j_{2} ; j_{1}^{\prime} I\right)\left(I M_{i} k_{I} q_{I} \mid I M_{f}\right) \sum_{k_{2} q_{2}}(-)^{-k_{2}}\left(\ell_{1}-\lambda_{1} \ell_{1}^{\prime} \lambda_{1}^{\prime} \mid k_{2} q_{2}\right) \\
& \times \quad\left(1-\mu_{1} k_{I} q_{I} \mid k_{2} q_{2}\right)\left\{\begin{array}{ccc}
j_{1} & j_{1}^{\prime} & k_{I} \\
\frac{1}{2} & \frac{1}{2} & 1 \\
\ell_{1} & \ell_{1}^{\prime} & k_{2}
\end{array}\right\} .
\end{aligned}
$$

The graphical representation of the C-G coefficients to be reduced in Eq. C.24 is shown in Figure C.2. The three C-G coefficients circled can be reduced using the relationship 
between Racah coefficients and C-G coefficients given in Eq. A.8

Term 3

$$
\begin{aligned}
& =(-)^{3 j_{1}-j_{1}^{\prime}+1-\ell_{1}^{\prime}+\ell_{1}} 6 \sqrt{6} \sqrt{4 \pi} \hat{I} \hat{j}_{1} \hat{j}_{1}^{\prime} \hat{\ell}_{1} \hat{\ell}_{1}^{\prime} R \int_{0}^{\infty} r_{1}^{3} d r_{1} u_{\ell_{1}^{\prime} j_{1}^{\prime}}\left(r_{1}\right) \sum_{K Q} \frac{f_{K}^{(t r)}\left(R, r_{1}\right)}{r_{p}^{2} \hat{K}} u_{\ell_{1} j_{1}}\left(r_{1}\right) \\
& \times \sum_{\substack{\mu \mu_{0} \mu_{1} \\
\alpha \beta}}(-)^{\mu+\mu_{1}}\left(1 \mu_{0} 1 \mu_{1} \mid 2-\mu\right) \tau_{1 \mu_{0}}\left(\sigma_{0}\right)(1 \alpha 1 \beta \mid 2 \mu) \sum_{C C^{\prime} \Gamma^{\prime}}\left(\ell_{1}^{\prime} 0 K 0 \mid C 0\right)\left(10 \ell_{1} 0 \mid C 0\right) \\
& \times \frac{Y_{C^{\prime} \Gamma^{\prime}}(\hat{\boldsymbol{R}})}{\hat{C}^{\prime}}\left(K Q 1 \alpha \mid C^{\prime} \Gamma^{\prime}\right)\left(K 010 \mid C^{\prime} 0\right) \sum_{k_{I} q_{I}}{\hat{k_{I}}}^{2} W\left(k_{I} j_{1} I j_{2} ; j_{1}^{\prime} I\right)\left(I M_{i} k_{I} q_{I} \mid I M_{f}\right) \\
& \times \sum_{k_{2} q_{2}}(-)^{q_{2}} \hat{k}_{2}\left(1 \beta k_{2}-q_{2} \mid K Q\right) W\left(1 \ell_{1} K \ell_{1}^{\prime} ; C k_{2}\right)\left(1-\mu_{1} k_{I} q_{I} \mid k_{2} q_{2}\right)\left\{\begin{array}{ccc}
j_{1} & j_{1}^{\prime} & k_{I} \\
\frac{1}{2} & \frac{1}{2} & 1 \\
\ell_{1} & \ell_{1}^{\prime} & k_{2}
\end{array}\right\} .
\end{aligned}
$$

Using this same reduction method twice more on the remaining C-G coefficients leads to the final folded potential given in Eq. 3.49

\section{Term 3}

$=(-)^{3 j_{1}-j_{1}^{\prime}-\ell_{1}^{\prime}+\ell_{1}} 30 \sqrt{6} \sqrt{4 \pi} \hat{I} \hat{j}_{1} \hat{j}_{1}^{\prime} \hat{\ell}_{1} \hat{\ell}_{1}^{\prime} R \int_{0}^{\infty} r_{1}^{3} d r_{1} u_{\ell_{1}^{\prime} j_{1}^{\prime}}\left(r_{1}\right) \sum_{k_{I} k_{2} C K C^{\prime}} \frac{f_{K}^{(t r)}\left(R, r_{1}\right)}{r_{p}^{2} \hat{C}^{\prime}}$

$\times u_{\ell_{1} j_{1}}\left(r_{1}\right)(-)^{K-k_{I}} \hat{k}_{2}^{2}\left(\ell_{1}^{\prime} 0 K 0 \mid C 0\right)\left(10 \ell_{1} 0 \mid C 0\right)\left(K 010 \mid C^{\prime} 0\right) W\left(11 C^{\prime} k_{2} ; 2 k_{I}\right)$

$\times W\left(1 \ell_{1} K \ell_{1}^{\prime} ; C k_{2}\right) W\left(k_{2} 1 C^{\prime} 1 ; K 2\right) \hat{k_{I}} W\left(k_{I} j_{1} I j_{2} ; j_{1}^{\prime} I\right)\left\{\begin{array}{ccc}j_{1} & j_{1}^{\prime} & k_{I} \\ \frac{1}{2} & \frac{1}{2} & 1 \\ \ell_{1} & \ell_{1}^{\prime} & k_{2}\end{array}\right\}\left\langle I, M_{f}\left|\boldsymbol{S}_{k_{I} C^{\prime}}\right| I, M_{i}\right\rangle$

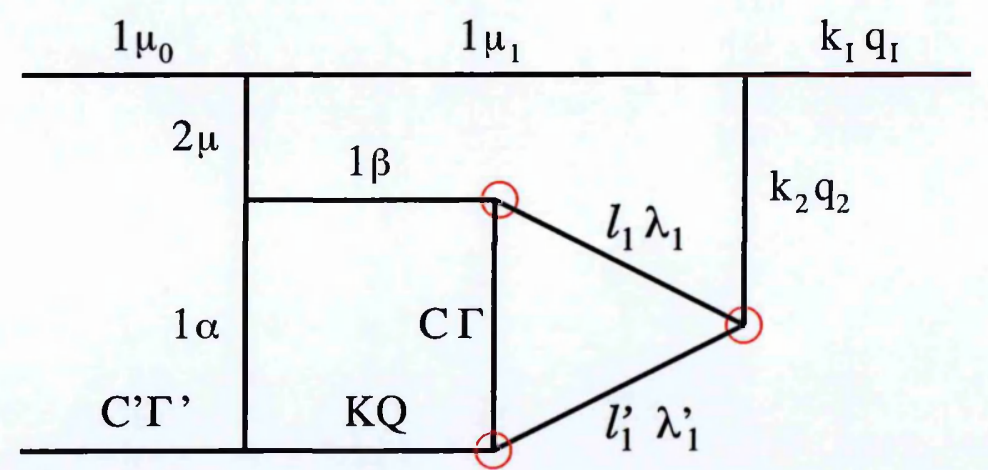

Figure C.2: The graphical representation of the C-G coefficients in Eq. C.24. 


\section{Appendix D}

\section{Folded Potential Formalism: Exchange Terms}

In this Appendix the details will be given of the folding potential formalism omitted from Chapter 5 for the central and tensor exchange terms in the NN interaction.

\section{D.1 Folding of Central Exchange NN Interaction Term}

Continuing the discussion from Section 5.2 the folding of the central exchange term in the NN interaction using the zero-range pseudo-potential $\hat{J}_{\sigma \sigma}(E) \delta\left(\boldsymbol{r}_{N}\right) \sigma_{0} \cdot \sigma_{1} P_{01}^{r}$ in the general exchange folding Eq. 5.1 gives

$$
\begin{aligned}
\left\langle\Psi_{I, M_{f}}\left(\boldsymbol{r}_{1}, \boldsymbol{r}_{2}\right)\left|\hat{J}_{\sigma \sigma}(E) \delta\left(\boldsymbol{r}_{N}\right) \boldsymbol{\sigma}_{0} \cdot \boldsymbol{\sigma}_{1} P_{01}^{r}\right| \Psi_{I, M_{i}}\left(\boldsymbol{r}_{1}, \boldsymbol{r}_{2}\right)\right\rangle \\
=(-)^{3 j_{1}-j_{1}^{\prime}} \hat{I} \hat{j}_{1} \hat{j}_{1}^{\prime} \int d \boldsymbol{r}_{1} \sum_{\lambda_{1}^{\prime} \lambda_{1} m_{s 1}^{\prime} m_{s 1}} Y_{\ell_{1}^{\prime} \lambda_{1}^{\prime}}^{*}\left(\hat{\boldsymbol{r}}_{1}\right) \chi_{\frac{1}{2} m_{s 1}^{\prime}}^{*} u_{\ell_{1}^{\prime} j_{1}^{\prime}}\left(r_{1}\right) \hat{J}_{\sigma \sigma}(E) \delta\left(\boldsymbol{r}_{p}\right) \\
\quad \times \sum_{\mu_{0}}(-)^{\mu_{0}} \sigma_{1 \mu_{0}}^{(0)} \sigma_{1-\mu_{0}}^{(1)} Y_{\ell_{1} \lambda_{1}}(\hat{\boldsymbol{R}}) \chi_{\frac{1}{2} m_{s 1}} u_{\ell_{1} j_{1}}(R)(-)^{1 / 2+m_{s 1}^{\prime}-\ell_{1}^{\prime}-\lambda_{1}} \\
\quad \times \sum_{k_{I} q_{I}} \hat{k}_{I}^{2} W\left(k_{I} j_{1} I j_{2} ; j_{1}^{\prime} I\right)\left(I M_{i} k_{I} q_{I} \mid I M_{f}\right) \sum_{k_{1} q_{1} k_{2} q_{2}}\left(\frac{1}{\frac{1}{2}} m_{s 1} \frac{1}{2}-m_{s 1}^{\prime} \mid k_{1} q_{1}\right) \\
\quad \times(-)^{k_{1}-k_{2}} \hat{k}_{1}\left(\ell_{1}-\lambda_{1} \ell_{1}^{\prime} \lambda_{1}^{\prime} \mid k_{2} q_{2}\right)\left(k_{1} q_{1} k_{I} q_{I} \mid k_{2} q_{2}\right)\left\{\begin{array}{ccc}
j_{1} & j_{1}^{\prime} & k_{I} \\
\frac{1}{2} & \frac{1}{2} & k_{1} \\
\ell_{1} & \ell_{1}^{\prime} & k_{2}
\end{array}\right\} P_{01}^{r},
\end{aligned}
$$

where we have used the exchange operator $P_{01}^{r}$ to change, $\boldsymbol{r}_{p} \rightarrow-\boldsymbol{r}_{p}$ or $\boldsymbol{r}_{1} \rightarrow \boldsymbol{R}$ and $\boldsymbol{R} \rightarrow \boldsymbol{r}_{1}$. In the calculation of the scattering amplitude the initial distorted wavefunction is on the left of this folded potential and is also acted upon by $P_{01}^{r}$, giving $P_{01}^{r} \chi_{\mu_{i}}^{(+)}(\boldsymbol{R}, \boldsymbol{k})=$ 
$\chi_{\mu_{i}}^{(+)}\left(\boldsymbol{r}_{1}, \boldsymbol{k}\right)$, however, using the properties of the delta function

$$
\int d \boldsymbol{r}_{1} f\left(\boldsymbol{r}_{1}\right) \delta\left(\boldsymbol{R}-\boldsymbol{r}_{1}\right)=f(\boldsymbol{R})
$$

to perform the integration changes $\chi_{\mu_{i}}^{(+)}\left(\boldsymbol{r}_{1}, \boldsymbol{k}\right)$ back to $\chi_{\mu_{i}}^{(+)}(\boldsymbol{R}, \boldsymbol{k})$. Using this result and substituting in for the matrix element $\left\langle\frac{1}{2} m_{s 1}^{\prime}\left|\sigma_{1-\mu_{0}}^{(1)}\right| \frac{1}{2} m_{s 1}\right\rangle$ using Eq. C.3 leads to

$$
\begin{aligned}
\left\langle\Psi_{I, M_{f}}\left(\boldsymbol{r}_{1}, \boldsymbol{r}_{2}\right)\left|\hat{J}_{\sigma \sigma}(Q) \delta\left(\boldsymbol{r}_{N}\right) \sigma_{0} \cdot \sigma_{1} P_{01}^{r}\right| \Psi_{I, M_{i}}\left(\boldsymbol{r}_{1}, \boldsymbol{r}_{2}\right)\right\rangle \\
=(-)^{3 j_{1}-j_{1}^{\prime}-\ell_{1}^{\prime}} \hat{J}_{\sigma \sigma}(Q) \frac{\sqrt{2}}{\sqrt{4 \pi}} \hat{I} \hat{j}_{1} \hat{j}_{1}^{\prime} \hat{\ell}_{1} \hat{\ell}_{1}^{\prime} u_{\ell_{1}^{\prime} j_{1}^{\prime}}\left(r_{1}\right) u_{\ell_{1} j_{1}}(R) \sum_{\mu_{0} \lambda_{1}^{\prime} \lambda_{1} m_{s 1}^{\prime} m_{s 1}} \sigma_{1 \mu_{0}}^{(0)}(-)^{\mu_{0}+1} \\
\quad \times\left(\frac{1}{2} m_{s 1} \frac{1}{2}-m_{s 1}^{\prime} \mid 1 \mu_{0}\right) \sum_{C \Gamma} \frac{Y_{C \Gamma}(\hat{\boldsymbol{R}})}{\hat{C}}\left(\ell_{1}-\lambda_{1} \ell_{1}^{\prime} \lambda_{1}^{\prime} \mid C-\Gamma\right)\left(\ell_{1}^{\prime} 0 \ell_{1} 0 \mid C 0\right) \\
\quad \times \sum_{k_{I} q_{I}} \hat{k}_{I}^{2} W\left(k_{I} j_{1} I j_{2} ; j_{1}^{\prime} I\right)\left(I M_{i} k_{I} q_{I} \mid I M_{f}\right) \sum_{k_{1} q_{1} k_{2} q_{2}}\left(\frac{1}{2} m_{s 1} \frac{1}{2}-m_{s 1}^{\prime} \mid k_{1} q_{1}\right) \\
\quad \times(-)^{k_{1}-k_{2}-q_{1}+q_{2}} \hat{k}_{1}\left(\ell_{1}-\lambda_{1} \ell_{1}^{\prime} \lambda_{1}^{\prime} \mid k_{2} q_{2}\right)\left(k_{1} q_{1} k_{I} q_{I} \mid k_{2} q_{2}\right)\left\{\begin{array}{ccc}
j_{1} & j_{1}^{\prime} & k_{I} \\
\frac{1}{2} & \frac{1}{2} & k_{1} \\
\ell_{1} & \ell_{1}^{\prime} & k_{2}
\end{array}\right\}
\end{aligned}
$$

where we have also combined the spherical harmonics using Eq. A.16. Using the orthogonality relation for C-G coefficients given in Eq. A.1 gives the final folded potential shown in Eq. 5.2

$$
\begin{aligned}
& \left\langle\Psi_{I, M_{f}}\left(\boldsymbol{r}_{1}, \boldsymbol{r}_{2}\right)\left|\hat{J}_{\sigma \sigma}(Q) \delta\left(\boldsymbol{r}_{N}\right) \boldsymbol{\sigma}_{0} \cdot \boldsymbol{\sigma}_{1} P_{01}^{r}\right| \Psi_{I, M_{i}}\left(\boldsymbol{r}_{1}, \boldsymbol{r}_{2}\right)\right\rangle \\
& =(-)^{3 j_{1}-j_{1}^{\prime}-\ell_{1}^{\prime}} \hat{J}_{\sigma \sigma}(Q) \sqrt{\frac{6}{4 \pi}} \hat{I_{j}} \hat{j}_{1} \hat{j}_{1}^{\prime} \hat{\ell}_{1} \hat{\ell}_{1}^{\prime} u_{\ell_{1}^{\prime} j_{1}^{\prime}}(R) u_{\ell_{1} j_{1}}(R) \sum_{k_{I} C} \hat{k}_{I} \\
& \quad \times W\left(k_{I} j_{1} I j_{2} ; j_{1}^{\prime} I\right) \frac{(-)^{C}}{\hat{C}}\left(\ell_{1} 0 \ell_{1}^{\prime} 0 \mid C 0\right)\left\{\begin{array}{ccc}
j_{1} & j_{1}^{\prime} & k_{I} \\
\frac{1}{2} & \frac{1}{2} & 1 \\
\ell_{1} & \ell_{1}^{\prime} & C
\end{array}\right\}\left\langle I, M_{f}\left|\boldsymbol{S}_{k_{I} C}\right| I, M_{i}\right\rangle .
\end{aligned}
$$

\section{D.2 Folding of Tensor Exchange NN Interaction Term}

In order to calculate the potentials derived from the folding of the tensor exchange term the operation of the solid harmonic $\mathscr{Y}_{2 \mu}\left(-\imath \nabla_{r}\right)$ on $u_{\ell j}(r) Y_{\ell m_{\ell}}(\hat{r})$ must be determined, where $u_{\ell j}(r)$ is a differentiable function of $r$ and $Y_{\ell m_{\ell}}(\hat{r})$ is a spherical harmonic. Starting 
from

$$
\nabla_{\mu} u_{\ell j}(r) Y_{\ell m_{\ell}}(\hat{\boldsymbol{r}})=\sum_{\ell^{\prime} m_{\ell}^{\prime}}\left(\ell m_{\ell} 1 \mu \mid \ell^{\prime} m_{\ell}^{\prime}\right)(-)^{\ell-\ell^{\prime}} \frac{\hat{\ell}}{\hat{\ell}^{\prime}}\left(\ell 010 \mid \ell^{\prime} 0\right)\left[-\hat{g}_{\ell^{\prime}, \ell} u_{\ell j}(r)\right] Y_{\ell^{\prime} m_{\ell}^{\prime}}(\hat{\boldsymbol{r}}),
$$

given in [89] and adapted from [42]. The parity C-G coefficient limits the values of $\ell^{\prime}$ to $\ell^{\prime}=\ell \pm 1$ and the differential operators $\hat{g}_{\ell \pm 1, \ell}$ are defined as

$$
\hat{g}_{\ell+1, \ell}=\frac{d}{d r}-\frac{\ell}{r} \quad, \quad \hat{g}_{\ell-1, \ell}=\frac{d}{d r}+\frac{\ell+1}{r} .
$$

Using Eq. 5.11 separating $\mathscr{Y}_{2 \mu}\left(-\imath \nabla_{\boldsymbol{r}}\right)$ into $\nabla_{\mu_{1}} \nabla_{\mu_{2}}$ allows us to write

$$
\begin{gathered}
\mathscr{Y}_{2 \mu}\left(-\imath \nabla_{\boldsymbol{r}}\right) u_{\ell j}(r) Y_{\ell m_{\ell}}(\hat{\boldsymbol{r}})=\frac{-\hat{2}}{\sqrt{4 \pi}} \sqrt{\frac{3}{2}} \sum_{\mu_{1} \mu_{2}}\left(1 \mu_{1} 1 \mu_{2} \mid 2 \mu\right) \sum_{\ell^{\prime} m_{\ell}^{\prime}}\left(\ell m_{\ell} 1 \mu_{1} \mid \ell^{\prime} m_{\ell}^{\prime}\right)(-)^{\ell-\ell^{\prime}} \frac{\hat{\ell}}{\hat{\ell}^{\prime}} \\
\times\left(\ell 010 \mid \ell^{\prime} 0\right) \nabla_{\mu_{2}}\left[-\hat{g}_{\ell^{\prime}, \ell} u_{\ell j}(r)\right] Y_{\ell^{\prime} m_{\ell}^{\prime}}(\hat{\boldsymbol{r}}) .
\end{gathered}
$$

Using Eq. D.5 again leads to

$$
\begin{aligned}
& \mathscr{Y}_{2 \mu}(\left.-\imath \nabla_{\boldsymbol{r}}\right) u_{\ell j}(r) Y_{\ell m_{\ell}}(\hat{\boldsymbol{r}})=\frac{-\hat{2}}{\sqrt{4 \pi}} \sqrt{\frac{3}{2}} \sum_{\mu_{1} \mu_{2}}\left(1 \mu_{1} 1 \mu_{2} \mid 2 \mu\right) \sum_{\ell^{\prime} m_{\ell}^{\prime} \ell^{\prime \prime} m_{\ell}^{\prime \prime}}\left(\ell m_{\ell} 1 \mu_{1} \mid \ell^{\prime} m_{\ell}^{\prime}\right) \\
& \quad \times\left(\ell^{\prime} m_{\ell}^{\prime} 1 \mu_{2} \mid \ell^{\prime \prime} m_{\ell}^{\prime \prime}\right)(-)^{\ell-\ell^{\prime \prime}} \frac{\hat{\ell}}{\hat{\ell}^{\prime \prime}}\left(\ell 010 \mid \ell^{\prime} 0\right)\left(\ell^{\prime} 010 \mid \ell^{\prime \prime} 0\right)\left[\hat{g}_{\ell^{\prime \prime}, \ell^{\prime}} \hat{g}_{\ell^{\prime}, \ell} u_{\ell j}(r)\right] Y_{\ell^{\prime \prime} m_{\ell}^{\prime \prime}}(\hat{\boldsymbol{r}}),
\end{aligned}
$$

coupling the three red C-G coefficients using Eq. A.8 leads to

$$
\begin{aligned}
& \mathscr{Y} 2 \mu\left(-\imath \nabla_{\boldsymbol{r}}\right) u_{\ell j}(r) Y_{\ell m_{\ell}}(\hat{\boldsymbol{r}})=\frac{-\hat{2}}{\sqrt{4 \pi}} \sqrt{\frac{3}{2}} \sum_{\ell^{\prime} \ell^{\prime \prime} m_{\ell}^{\prime \prime}}(-)^{\ell-\ell^{\prime \prime}} \frac{\hat{2} \hat{\ell} \hat{\ell}^{\prime}}{\hat{\ell}^{\prime \prime}} W\left(\ell 1 \ell^{\prime \prime} 1 ; \ell^{\prime} 2\right) \\
& \times\left(\ell m_{\ell} 2 \mu \mid \ell^{\prime \prime} m_{\ell}^{\prime \prime}\right)\left(\ell 010 \mid \ell^{\prime} 0\right)\left(\ell^{\prime} 010 \mid \ell^{\prime \prime} 0\right)\left[\hat{g}_{\ell^{\prime \prime}, \ell^{\prime}} \hat{g}_{\ell^{\prime}, \ell} u_{\ell j}(r)\right] Y_{\ell^{\prime \prime} m_{\ell}^{\prime \prime}}(\hat{\boldsymbol{r}}) .
\end{aligned}
$$

The combination of parity coefficients determine that $\ell^{\prime \prime}=\ell$ and $\ell \pm 2$. The allowed combinations of differential operators are

$$
\begin{aligned}
\hat{\mathscr{O}}_{\ell-2, \ell} & =\hat{g}_{\ell-2, \ell-1} \hat{g}_{\ell-1, \ell}=\frac{d^{2}}{d r^{2}}+\frac{2 \ell+1}{r} \frac{d}{d r}+\frac{\ell^{2}-1}{r^{2}} \\
\hat{\mathscr{O}}_{\ell, \ell} & =\hat{g}_{\ell, \ell-1} \hat{g}_{\ell-1, \ell}=\hat{g}_{\ell, \ell+1} \hat{g}_{\ell+1, \ell}=\frac{d^{2}}{d r^{2}}+\frac{2}{r} \frac{d}{d r}-\frac{\ell(\ell+1)}{r^{2}} \\
\hat{\mathscr{O}}_{\ell+2, \ell} & =\hat{g}_{\ell+2, \ell+1} \hat{g}_{\ell+1, \ell}=\frac{d^{2}}{d r^{2}}-\frac{2 \ell+1}{r} \frac{d}{d r}+\frac{\ell(\ell+2)}{r^{2}}
\end{aligned}
$$


Now using the $\hat{\mathscr{O}}$ operator and the relationship between C-G and Racah coefficients to sum over $\ell^{\prime}$ gives

$$
\mathscr{Y}_{2 \mu}\left(-\imath \nabla_{\boldsymbol{r}}\right) u_{\ell j}(r) Y_{\ell m_{\ell}}(\hat{\boldsymbol{r}})=\frac{-\hat{2}}{\sqrt{4 \pi}} \sum_{\ell^{\prime \prime} m_{\ell}^{\prime \prime}}\left(\ell m_{\ell} 2 \mu \mid \ell^{\prime \prime} m_{\ell}^{\prime \prime}\right)\left(20 \ell^{\prime \prime} 0 \mid \ell 0\right)\left[\hat{\mathscr{O}}_{\ell^{\prime \prime}, \ell} u_{\ell j}(r)\right] Y_{\ell^{\prime \prime} m_{\ell}^{\prime \prime}}(\hat{\boldsymbol{r}})
$$

Now that the differential operators have been defined the details of the folding formalism for the three different tensor exchange terms discussed in Section 5.3 can be developed. This will be done in the next three sections.

\section{D.3 NN Tensor Exchange Term A Folding Formalism}

In this term the solid spherical harmonic acts on the components of the final valence nucleon wavefunction, $\left[\mathscr{Y}_{2 \mu}\left(-\imath \nabla_{\boldsymbol{R}}\right) Y_{\ell_{1}^{\prime} \lambda_{1}^{\prime}}^{*}(\hat{\boldsymbol{R}}) u_{\ell_{1}^{\prime} j_{1}^{\prime}}(R)\right]$. Continuing the discussion from Section 5.3.1 and inserting this term into the general folding expression Eq. 5.18 leads to

$$
\begin{aligned}
\text { Term } & \mathbf{A}=(-)^{3 j_{1}-j_{1}^{\prime}-\ell_{1}^{\prime}} \hat{J}_{t r}(Q) 3 \sqrt{2} \sqrt{\frac{8 \pi}{5}} \hat{I} \hat{j}_{1} \hat{j}_{1}^{\prime} \sum(-)^{\mu+\mu_{1}}\left(1 \mu_{0} 1 \mu_{1} \mid 2-\mu\right) \tau_{1 \mu_{0}}\left(\boldsymbol{\sigma}_{0}\right) \\
= & (-)^{3 j_{1}-j_{1}^{\prime}-\ell_{1}^{\prime}} \hat{J}_{t r}(Q) 3 \sqrt{2} \sqrt{\frac{8 \pi}{5}} \hat{I} \hat{j}_{1} \hat{j}_{1}^{\prime} \sum_{\mu \mu_{0} \mu_{1}}(-)^{\mu+\mu_{1}}\left(1 \mu_{0} 1 \mu_{1} \mid 2-\mu\right) \tau_{1 \mu_{0}}\left(\boldsymbol{\sigma}_{0}\right) \\
& \times \sum_{\lambda_{1}^{\prime} \lambda_{1}}\left[\mathscr{Y}_{2 \mu}\left(-\imath \nabla_{\boldsymbol{R}}\right) Y_{\ell_{1}^{\prime} \lambda_{1}^{\prime}}^{*}(\hat{\boldsymbol{R}}) u_{\ell_{1}^{\prime} j_{1}^{\prime}}(R)\right](-)^{-\lambda_{1}} Y_{\ell_{1} \lambda_{1}}(\hat{\boldsymbol{R}}) u_{\ell_{1} j_{1}}(R) \\
& \times \sum_{k_{I} q_{I}} \hat{k}_{I}^{2} W\left(k_{I} j_{1} I j_{2} ; j_{1}^{\prime} I\right)\left(I M_{i} k_{I} q_{I} \mid I M_{f}\right) \sum_{k_{2} q_{2}}(-)^{-k_{2}} \\
& \times\left(\ell_{1}-\lambda_{1} \ell_{1}^{\prime} \lambda_{1}^{\prime} \mid k_{2} q_{2}\right)\left(1-\mu_{1} k_{I} q_{I} \mid k_{2} q_{2}\right)\left\{\begin{array}{ccc}
j_{1} & j_{1}^{\prime} & k_{I} \\
\frac{1}{2} & \frac{1}{2} & 1 \\
\ell_{1} & \ell_{1}^{\prime} & k_{2}
\end{array}\right\} .
\end{aligned}
$$

Using Eq. D.13 for $\mathscr{Y}_{2 \mu}\left(-\imath \nabla_{\boldsymbol{R}}\right) Y_{\ell_{1}^{\prime} \lambda_{1}^{\prime}}^{*}(\hat{\boldsymbol{R}}) u_{\ell_{1}^{\prime} j_{1}^{\prime}}(R)$ and Eq. A.16 for the coupling of two spherical harmonics gives 


\section{Term A}

$$
\begin{aligned}
& =(-)^{3 j_{1}-i_{1}^{\prime}-\ell_{1}^{\prime}} \hat{J}_{t r}(Q) 3 \sqrt{2} \sqrt{\frac{8 \pi}{5}} \hat{\tilde{I}}_{\hat{j}_{1}} \hat{j}_{1}^{\prime} u_{\ell_{1} j_{i}}(R) \sum_{\mu \mu_{0} \mu_{1}}(-)^{\mu+\mu_{1}}\left(1 \mu_{0} 1 \mu_{1} \mid 2-\mu\right) \tau_{1 \mu_{0}}\left(\boldsymbol{\sigma}_{0}\right) \\
& \times \sum_{\lambda_{1}^{\prime} \lambda_{1}} \frac{-\hat{2}}{\sqrt{4 \pi}} \sum_{L M}\left(\ell_{1}^{\prime}-\lambda_{1}^{\prime} 2 \mu \mid L M\right)\left(20 L 0 \mid \ell_{1}^{\prime} 0\right)\left[\hat{\mathscr{O}}_{L, \ell_{1}^{\prime}} u_{\ell_{1}^{\prime} j_{1}^{\prime}}(R)\right] \frac{\hat{\ell_{1}} \hat{L}}{\sqrt{4 \pi}} \sum_{C \Gamma} \frac{Y_{C \Gamma}(\hat{\boldsymbol{R}})}{C} \\
& \times\left(\ell_{1} \lambda_{1} L M \mid C \Gamma\right)\left(\ell_{1} 0 L 0 \mid C 0\right) \sum_{k_{I} q_{I}} \hat{k}_{I}^{2} W\left(k_{I} j_{1} I j_{2} ; j_{1}^{\prime} I\right)\left(I M_{i} k_{I} q_{I} \mid I M_{f}\right) \sum_{k_{2} q_{2}}(-)^{-k_{2}+q_{2}} \\
& \times\left(\ell_{1}-\lambda_{1} \ell_{1}^{\prime} \lambda_{1}^{\prime} \mid k_{2} q_{2}\right)\left(1-\mu_{1} k_{I} q_{I} \mid k_{2} q_{2}\right)\left\{\begin{array}{ccc}
j_{1} & j_{1}^{\prime} & k_{I} \\
\frac{1}{2} & \frac{1}{2} & 1 \\
\ell_{1} & \ell_{1}^{\prime} & k_{2}
\end{array}\right\} .
\end{aligned}
$$

The graphical representation of the five C-G coefficients in Eq. D.15 to be coupled is shown in Figure D.1. The three C-G coefficients circled in Figure D.1 can be reduced using the relationship between Racah coefficients and $\mathrm{C}-\mathrm{G}$ coefficients given in Eq. A.8, this leads to

\section{Term A}

$$
\begin{aligned}
& =(-)^{1+3 j_{1}-j_{1}^{\prime}-\ell_{1}^{\prime}} \hat{J}_{t r}(Q) \frac{6}{\sqrt{4 \pi}} \hat{I} \hat{\ell}_{1} \hat{j}_{1} \hat{j}_{1}^{\prime} u_{\ell_{1} j_{1}}(R) \sum_{\mu \mu_{0} \mu_{1}}(-)^{\mu+\mu_{1}}\left(1 \mu_{0} 1 \mu_{1} \mid 2-\mu\right) \tau_{1 \mu_{0}}\left(\boldsymbol{\sigma}_{0}\right) \\
& \times \sum_{L C \Gamma} \frac{\hat{L}^{2}}{\hat{C}}\left(20 L 0 \mid \ell_{1}^{\prime} 0\right)\left[\hat{\mathscr{O}}_{L, \ell_{1}^{\prime}} u_{\ell_{1}^{\prime} j_{1}^{\prime}}(R)\right] Y_{C \Gamma}(\hat{\boldsymbol{R}})\left(\ell_{1} 0 L 0 \mid C 0\right) \sum_{k_{I} q_{I}} \hat{k}_{I}^{2} W\left(k_{I} j_{1} I j_{2} ; j_{1}^{\prime} I\right) \\
& \times\left(I M_{i} k_{I} q_{I} \mid I M_{f}\right) \sum_{k_{2} q_{2}}(-)^{-k_{2}+q_{2}} \hat{k}_{2}\left(2 \mu k_{2}-q_{2} \mid C \Gamma\right) W\left(2 \ell_{1}^{\prime} C \ell_{1} ; L k_{2}\right) \\
& \times\left(1-\mu_{1} k_{I} q_{I} \mid k_{2} q_{2}\right)\left\{\begin{array}{ccc}
j_{1} & j_{1}^{\prime} & k_{I} \\
\frac{1}{2} & \frac{1}{2} & 1 \\
\ell_{1} & \ell_{1}^{\prime} & k_{2}
\end{array}\right\} .
\end{aligned}
$$

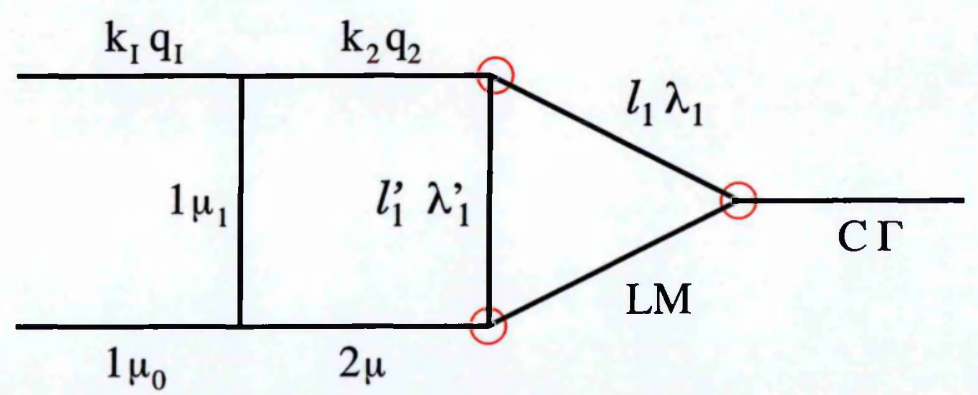

Figure D.1: The graphical representation of the C-G coefficients in Eq. D.15. 
The remaining three C-G coefficients shown in red can also be reduced using Eq. A.8 to give the final folded potential of Eq. 5.20

Term A

$$
\begin{aligned}
& =(-)^{1+3 j_{1}-j_{1}^{\prime}-\ell_{1}^{\prime}} \hat{J}_{t r}(Q) \frac{6 \sqrt{5}}{\sqrt{4 \pi}} \hat{I} \hat{\ell}_{1} \hat{j}_{1} \hat{j}_{1}^{\prime} u_{\ell_{1} j_{1}}(R) \sum_{k_{I} k_{2} L C}\left[\hat{\mathscr{O}}_{L, \ell_{1}^{\prime}} u_{\ell_{1}^{\prime} j_{1}^{\prime}}(R)\right] \\
& \times(-)^{-k_{2}-k_{I}-C} \frac{\hat{L}^{2}{\hat{k_{2}}}^{2}}{\hat{C}}\left(20 L 0 \mid \ell_{1}^{\prime} 0\right)\left(\ell_{1} 0 L 0 \mid C 0\right) \hat{k}_{I} W\left(k_{I} j_{1} I j_{2} ; j_{1}^{\prime} I\right) \\
& \times W\left(2 \ell_{1}^{\prime} C \ell_{1} ; L k_{2}\right) W\left(11 C k_{2} ; 2 i\right)\left\{\begin{array}{ccc}
j_{1} & j_{1}^{\prime} & k_{I} \\
\frac{1}{2} & \frac{1}{2} & 1 \\
\ell_{1} & \ell_{1}^{\prime} & k_{2}
\end{array}\right\}\left\langle I, M_{f}\left|S_{k_{I} C}\right| I, M_{i}\right\rangle .
\end{aligned}
$$

\section{D.4 NN Tensor Exchange Term B Folding Formalism}

This term from the tensor exchange folding is the one in which the solid spherical harmonic acts on the initial distorted wavefunction, $Y_{\ell_{1}^{\prime} \lambda_{1}^{\prime}}^{*}(\hat{\boldsymbol{R}}) u_{\ell_{1}^{\prime} j_{1}^{\prime}}(R)\left[\mathscr{Y}_{2 \mu}\left(-\imath \nabla_{\boldsymbol{R}}\right) \chi_{\mu_{i}}^{(+)}(\boldsymbol{R}, \boldsymbol{k})\right]$, . Inserting this term into the general folding equation for exchange terms, Eq. 5.18, gives

\section{Term B}

$$
\begin{aligned}
& =(-)^{3 j_{1}-j_{1}^{\prime}-\ell_{1}^{\prime}} \hat{J}_{t r}(Q) 3 \sqrt{2} \sqrt{\frac{8 \pi}{5}} \hat{I} \hat{j}_{1} \hat{j}_{1}^{\prime} \sum_{\mu \mu_{0} \mu_{1}}(-)^{\mu+\mu_{1}}\left(1 \mu_{0} 1 \mu_{1} \mid 2-\mu\right) \tau_{1 \mu_{0}}\left(\boldsymbol{\sigma}_{0}\right) \\
& \times \sum_{\lambda_{1}^{\prime} \lambda_{1}} Y_{\ell_{1}^{\prime} \lambda_{1}^{\prime}}^{*}(\hat{\boldsymbol{R}}) u_{\ell_{1}^{\prime} j_{1}^{\prime}}(R)(-)^{-\lambda_{1}} Y_{\ell_{1} \lambda_{1}}(\hat{\boldsymbol{R}}) u_{\ell_{1} j_{1}}(R) \sum_{k_{I} q_{I}} \hat{k}_{I}^{2} W\left(k_{I} j_{1} I j_{2} ; j_{1}^{\prime} I\right) \\
& \times\left(I M_{i} k_{I} q_{I} \mid I M_{f}\right) \sum_{k_{2} q_{2}}(-)^{-k_{2}}\left(\ell_{1}-\lambda_{1} \ell_{1}^{\prime} \lambda_{1}^{\prime} \mid k_{2} q_{2}\right)\left(1-\mu_{1} k_{I} q_{I} \mid k_{2} q_{2}\right)\left\{\begin{array}{ccc}
j_{1} & j_{1}^{\prime} & k_{I} \\
\frac{1}{2} & \frac{1}{2} & 1 \\
\ell_{1} & \ell_{1}^{\prime} & k_{2}
\end{array}\right\} \\
& \times\left[\mathscr{Y}_{2 \mu}\left(-\imath \nabla_{R}\right) \chi_{\mu_{i}}^{(+)}(\boldsymbol{R}, k)\right] .
\end{aligned}
$$

Using Eq. A.16 to couple the two spherical harmonics yields the following 


\section{Term B}

$$
\begin{aligned}
& =(-)^{3 j_{1}-j_{1}^{\prime}-\ell_{1}^{\prime}} \hat{J}_{t r}(Q) 6 \sqrt{\frac{4 \pi}{5}} \hat{I} \hat{j}_{1} \hat{j}_{1}^{\prime} u_{\ell_{1}^{\prime} j_{1}^{\prime}}(R) u_{\ell_{1} j_{1}}(R) \sum_{\mu \mu_{0} \mu_{1}}(-)^{\mu+\mu_{1}}\left(1 \mu_{0} 1 \mu_{1} \mid 2-\mu\right) \tau_{1 \mu_{0}}\left(\boldsymbol{\sigma}_{0}\right) \\
& \times \sum_{\lambda_{1}^{\prime} \lambda_{1} C \Gamma}(-)^{\lambda_{1}^{\prime}-\lambda_{1}} \frac{\hat{\ell}_{1}^{\prime} \hat{\ell}_{1}}{\sqrt{4 \pi}} \frac{Y_{C \Gamma}(\hat{\boldsymbol{R}})}{\hat{C}}\left(\ell_{1} \lambda_{1} \ell_{1}^{\prime}-\lambda_{1}^{\prime} \mid C \Gamma\right)\left(\ell_{1} 0 \ell_{1}^{\prime} 0 \mid C 0\right) \sum_{k_{I} q_{I}} \hat{k}_{I}^{2} W\left(k_{I} j_{1} I j_{2} ; j_{1}^{\prime} I\right) \\
& \times \quad\left(I M_{i} k_{I} q_{I} \mid I M_{f}\right) \sum_{k_{2} q_{2}}(-)^{-k_{2}}\left(\ell_{1}-\lambda_{1} \ell_{1}^{\prime} \lambda_{1}^{\prime} \mid k_{2} q_{2}\right)\left(1-\mu_{1} k_{I} q_{I} \mid k_{2} q_{2}\right)\left\{\begin{array}{ccc}
j_{1} & j_{1}^{\prime} & k_{I} \\
\frac{1}{2} & \frac{1}{2} & 1 \\
\ell_{1} & \ell_{1}^{\prime} & k_{2}
\end{array}\right\} \\
& \times\left[\mathscr{Y}_{2 \mu}\left(-\imath \nabla_{\boldsymbol{R}}\right) \chi_{\mu_{i}}^{(+)}(\boldsymbol{R}, \boldsymbol{k})\right] .
\end{aligned}
$$

Using the orthogonality of the C-G coefficients given in Eq. A.1 this reduces to the final folded potential given in Eq. 5.27

\section{Term B}

$$
\begin{aligned}
& =(-)^{1+3 j_{1}-j_{1}^{\prime}-\ell_{1}^{\prime}} \hat{J}_{t r}(Q) 2 \hat{I} \hat{j}_{1} \hat{j}_{1}^{\prime} \hat{\ell}_{1} \hat{\ell}_{1}^{\prime} u_{\ell_{1}^{\prime} j_{1}^{\prime}}(R) u_{\ell_{1} j_{1}}(R) \\
& \times \sum_{k_{I} C}(-)^{k_{I}-C}\left(\ell_{1} 0 \ell_{1}^{\prime} 0 \mid C 0\right) \hat{k}_{I} W\left(k_{I} j_{1} I j_{2} ; j_{1}^{\prime} I\right)\left\{\begin{array}{ccc}
j_{1} & j_{1}^{\prime} & k_{I} \\
\frac{1}{2} & \frac{1}{2} & 1 \\
\ell_{1} & \ell_{1}^{\prime} & C
\end{array}\right\} \\
& \times \sum_{\Gamma q_{I} \mu_{0} \mu_{1} \mu}(-1)^{\mu_{1}}\left(C \Gamma k_{I} q_{I} \mid 1 \mu_{1}\right) Y_{C \Gamma}(\hat{\boldsymbol{R}})\left\langle I, M_{f}\left|\tau_{k_{I} q_{I}}(\boldsymbol{I})\right| I, M_{i}\right\rangle\left(1 \mu_{0} 2 \mu \mid 1-\mu_{1}\right) \tau_{1 \mu_{0}}\left(\boldsymbol{\sigma}_{0}\right) \\
& \times\left[\mathscr{Y}_{2 \mu}\left(-\imath \nabla_{\boldsymbol{R}}\right) \chi_{\mu_{i}}^{(+)}(\boldsymbol{R}, \boldsymbol{k})\right] .
\end{aligned}
$$

\section{D.5 NN Tensor Exchange Term C Folding Formalism}

This is the part of the tensor exchange term where the solid spherical harmonic is separated into two grads, one of which acts on the components of the final valence nucleon wavefunction and one of which acts on the initial distorted wavefunction. This term can be folded using Eq. 5.18 


\section{Term C}

$$
\begin{aligned}
& =(-)^{3 j_{1}-j_{1}^{\prime}-\ell_{1}^{\prime}} \hat{J}_{t r}(Q) 3 \sqrt{2} \sqrt{\frac{8 \pi}{5}} \hat{I} \hat{j}_{1} \hat{j}_{1}^{\prime} \sum_{\mu \mu_{0} \mu_{1}}(-)^{\mu+\mu_{1}}\left(1 \mu_{0} 1 \mu_{1} \mid 2-\mu\right) \tau_{1 \mu_{0}}\left(\boldsymbol{\sigma}_{0}\right) \\
& \times \sum_{\lambda_{1}^{\prime} \lambda_{1}}(-2) \frac{\hat{2}}{\sqrt{4 \pi}} \sqrt{\frac{3}{2}} \sum_{\mu_{1} \mu_{2}}\left(1 \mu_{2} 1 \mu_{3} \mid 2 \mu\right)\left[\nabla_{\mu_{2}} Y_{\ell_{1}^{\prime} \lambda_{1}^{\prime}}^{*}(\hat{\boldsymbol{R}}) u_{\ell_{1}^{\prime} j_{1}^{\prime}}(R)\right](-)^{-\lambda_{1}} Y_{\ell_{1} \lambda_{1}}(\hat{\boldsymbol{R}}) \\
& \times u_{\ell_{1} j_{1}}(R) \sum_{k_{I} q_{I}}{\hat{k_{I}}}^{2} W\left(k_{I} j_{1} I j_{2} ; j_{1}^{\prime} I\right)\left(I M_{i} k_{I} q_{I} \mid I M_{f}\right) \sum_{k_{2} q_{2}}(-)^{-k_{2}} \\
& \times\left(\ell_{1}-\lambda_{1} \ell_{1}^{\prime} \lambda_{1}^{\prime} \mid k_{2} q_{2}\right)\left(1-\mu_{1} k_{I} q_{I} \mid k_{2} q_{2}\right)\left\{\begin{array}{ccc}
j_{1} & j_{1}^{\prime} & k_{I} \\
\frac{1}{2} & \frac{1}{2} & 1 \\
\ell_{1} & \ell_{1}^{\prime} & k_{2}
\end{array}\right\}\left[\nabla_{\mu_{3}} \chi_{\mu_{i}}^{(+)}(\boldsymbol{R}, k)\right] .
\end{aligned}
$$

Inserting Eq. D.5 for $\nabla_{\mu_{2}} Y_{\ell_{1}^{\prime} \lambda_{1}^{\prime}}^{*}(\hat{\boldsymbol{R}}) u_{\ell_{1}^{\prime} j_{1}^{\prime}}(R)$ and coupling the two remaining spherical harmonics using Eq. A.16 leads to

Term C

$$
\begin{aligned}
& =(-)^{3 j_{1}-j_{1}^{\prime}-\ell_{1}^{\prime}} \hat{J}_{t r}(Q) 3 \sqrt{2} \sqrt{\frac{8 \pi}{5}} \hat{I}_{j_{1}} \hat{j}_{1}^{\prime} u_{\ell_{1} j_{1}}(R) \sum_{\mu \mu_{0} \mu_{1}}(-)^{\mu+\mu_{1}}\left(1 \mu_{0} 1 \mu_{1} \mid 2-\mu\right) \tau_{1 \mu_{0}}\left(\boldsymbol{\sigma}_{0}\right) \\
& \times \sum_{\lambda_{1}^{\prime} \lambda_{1}} \frac{-2 \sqrt{5}}{\sqrt{4 \pi}} \sqrt{\frac{3}{2}} \sum_{\mu_{1} \mu_{2}}\left(1 \mu_{2} 1 \mu_{3} \mid 2 \mu\right) \sum_{L M}\left(\ell_{1}^{\prime}-\lambda_{1}^{\prime} 1 \mu_{2} \mid L M\right)(-)^{\ell_{1}^{\prime}-L} \frac{\hat{\ell}_{1}^{\prime}}{\hat{L}}\left(\ell_{1}^{\prime} 010 \mid L 0\right)\left[-\hat{g}_{L, \ell_{1}^{\prime}} u_{\ell_{1}^{\prime} j_{1}^{\prime}}(R)\right] \\
& \times \frac{\hat{\ell}_{1} \hat{L}}{\sqrt{4 \pi}} \sum_{C \Gamma} \frac{Y_{C \Gamma}(\hat{\boldsymbol{R}})}{\hat{C}}\left(\ell_{1} \lambda_{1} L M \mid C \Gamma\right)\left(\ell_{1} 0 L 0 \mid C 0\right) \sum_{k_{I} q_{I}} \hat{k}_{I}^{2} W\left(k_{I} j_{1} I j_{2} ; j_{1}^{\prime} I\right)\left(I M_{i} k_{I} q_{I} \mid I M_{f}\right) \\
& \times \sum_{k_{2} q_{2}}(-)^{-k_{2}+q_{2}}\left(\ell_{1}-\lambda_{1} \ell_{1}^{\prime} \lambda_{1}^{\prime} \mid k_{2} q_{2}\right)\left(1-\mu_{1} k_{I} q_{I} \mid k_{2} q_{2}\right)\left\{\begin{array}{ccc}
j_{1} & j_{1}^{\prime} & k_{I} \\
\frac{1}{2} & \frac{1}{2} & 1 \\
\ell_{1} & \ell_{1}^{\prime} & k_{2}
\end{array}\right\}\left[\nabla_{\mu_{3}} \chi_{\mu_{i}}^{(+)}(\boldsymbol{R}, \boldsymbol{k})\right] .
\end{aligned}
$$

The graphical representation of the C-G coefficients to be reduced in Eq. D.22 are shown in Figure D.2. The three C-G coefficients circled in red can be reduced to give a Racah coefficient using Eq. A.8 


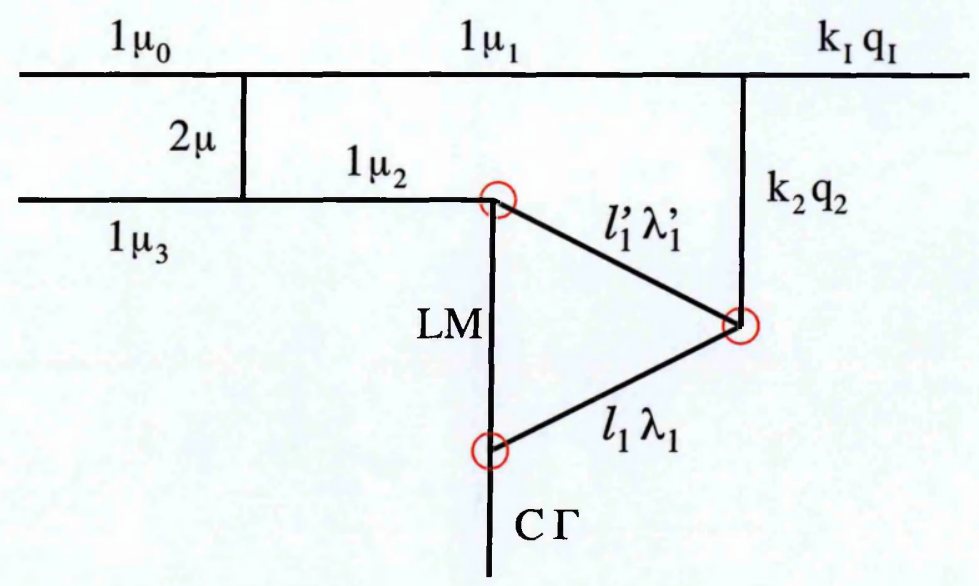

Figure D.2: The graphical representation of the C-G coefficients in Eq. D.22.

\section{Term C}

$$
\begin{aligned}
& =(-)^{3 j_{1}-j_{1}^{\prime}} \hat{J}_{t r}(Q) 6 \sqrt{\frac{6}{4 \pi}} \hat{I}_{j_{1}} \hat{j}_{1}^{\prime} \hat{\ell}_{1} \hat{\ell}_{1}^{\prime} u_{\ell_{1} j_{1}}(R) \sum_{\mu \mu_{0} \mu_{1}}(-)^{\mu+\mu_{1}}\left(1 \mu_{0} 1 \mu_{1} \mid 2-\mu\right) \tau_{1 \mu_{0}}\left(\boldsymbol{\sigma}_{0}\right) \\
& \times \sum_{\mu_{1} \mu_{2} L}(-)^{-L} \hat{L}\left(1 \mu_{2} 1 \mu_{3} \mid 2 \mu\right)\left(\ell_{1}^{\prime} 010 \mid L 0\right)\left[\hat{g}_{L, \ell_{1}^{\prime}} u_{\ell_{1}^{\prime} j_{1}^{\prime}}(R)\right] \sum_{C \Gamma} \frac{Y_{C \Gamma}(\hat{\boldsymbol{R}})}{\hat{C}}\left(\ell_{1} 0 L 0 \mid C 0\right) \\
& \times \sum_{k_{I} q_{I}}{\hat{k_{I}}}^{2} W\left(k_{I} j_{1} I \dot{j}_{2} ; j_{1}^{\prime} I\right)\left(I M_{i} k_{I} q_{I} \mid I M_{f}\right) \sum_{k_{2} q_{2}}(-)^{-k_{2}+q_{2}} \hat{k}_{2}\left(1 \mu_{2} k_{2}-q_{2} \mid C \Gamma\right) \\
& \times W\left(1 \ell_{1}^{\prime} C \ell_{1} ; L k_{2}\right)\left(1-\mu_{1} k_{I} q_{I} \mid k_{2} q_{2}\right)\left\{\begin{array}{ccc}
j_{1} & j_{1}^{\prime} & k_{I} \\
\frac{1}{2} & \frac{1}{2} & 1 \\
\ell_{1} & \ell_{1}^{\prime} & k_{2}
\end{array}\right\}\left[\nabla_{\mu_{3}} \chi_{\mu_{i}}^{(+)}(\boldsymbol{R}, k)\right] .
\end{aligned}
$$

The C-G coefficients and phase factors highlighted in red can be reduced using the relationships between $\mathrm{C}-\mathrm{G}$ coefficients and 9-j symbols discussed in Section A.3 to give the following result

$$
\begin{array}{r}
\sum_{\mu \mu_{1} \mu_{2} q_{2}}(-)^{\mu+\mu_{1}+q_{2}}\left(1 \mu_{0} 1 \mu_{1} \mid 2-\mu\right)\left(1 \mu_{2} k_{2}-q_{2} \mid C \Gamma\right)\left(1-\mu_{1} k_{I} q_{I} \mid k_{2} q_{2}\right)\left(1 \mu_{2} 1 \mu_{3} \mid 2 \mu\right) \\
=\frac{5 \hat{C} \hat{k}_{2}}{\sqrt{3}}(-)^{k_{I}-k_{2}-C+\mu_{3}} \sum_{E \epsilon} \hat{E}\left(1 \mu_{0} C \Gamma \mid E \epsilon\right)\left(E \epsilon k_{I} q_{I} \mid 1-\mu_{3}\right)\left\{\begin{array}{ccc}
1 & C & E \\
1 & k_{2} & k_{I} \\
2 & 1 & 1
\end{array}\right\} .
\end{array}
$$

This reduces Eq. D.23 to 
Term C

$$
\begin{aligned}
& =(-)^{3 j_{1}-j_{1}^{\prime}} \hat{J}_{t r}(Q) 30 \sqrt{\frac{2}{4 \pi}} \hat{I}_{1}^{\prime} \hat{j}_{1}^{\prime} \hat{\ell}_{1} \hat{\ell}_{1}^{\prime} u_{\ell_{1} j_{1}}(R) \sum_{L C \Gamma \mu_{0} \mu_{3}} \tau_{1 \mu_{0}}\left(\boldsymbol{\sigma}_{0}\right) \hat{L}\left(\ell_{1}^{\prime} 010 \mid L 0\right) \\
& \times\left[\hat{g}_{L, \ell_{1}^{\prime}} u_{\ell_{1}^{\prime} j_{1}^{\prime}}(R)\right] Y_{C \Gamma}(\hat{\boldsymbol{R}})\left(\ell_{1} 0 L 0 \mid C 0\right) \sum_{k_{2} k_{I} q_{I}}(-)^{k_{I}-L-C+\mu_{3}} \hat{k}_{2}^{2}{\hat{k_{I}}}_{I}^{2} W\left(k_{I} j_{1} I j_{2} ; j_{1}^{\prime} I\right) \\
& \times\left(I M_{i} k_{I} q_{I} \mid I M_{f}\right) W\left(1 \ell_{1}^{\prime} C \ell_{1} ; L k_{2}\right)\left\{\begin{array}{ccc}
j_{1} & j_{1}^{\prime} & k_{I} \\
\frac{1}{2} & \frac{1}{2} & 1 \\
\ell_{1} & \ell_{1}^{\prime} & k_{2}
\end{array}\right\} \sum_{E \epsilon} \hat{E}\left(1 \mu_{0} C \Gamma \mid E \epsilon\right) \\
& \times\left(E \epsilon k_{I} q_{I} \mid 1-\mu_{3}\right)\left\{\begin{array}{ccc}
1 & C & E \\
1 & k_{2} & k_{I} \\
2 & 1 & 1
\end{array}\right\}\left[\nabla_{\mu_{3}} \chi_{\mu_{i}}^{(+)}(\boldsymbol{R}, k)\right] .
\end{aligned}
$$

The remaining $\mathrm{C}-\mathrm{G}$ coefficients can be rearranged using the relations given in Appendix A.1 to give the final folded potential in Eq. 5.35

Term C

$$
\begin{aligned}
& =(-)^{3 j_{1}-j_{1}^{\prime}} \hat{J}_{t r}(Q) 30 \sqrt{\frac{6}{4 \pi}} \hat{I} \hat{j}_{1} \hat{j}_{1}^{\prime} \hat{\ell}_{1} \hat{\ell}_{1}^{\prime} u_{\ell_{1} j_{1}}(R) \sum_{k_{I} k_{2} L C E}\left[\hat{g}_{L, \ell_{1}^{\prime}} u_{\ell_{1}^{\prime} j_{1}^{\prime}}(R)\right] \\
& \times(-)^{k_{I}-L} \hat{L} \hat{k}_{2}^{2}\left(\ell_{1}^{\prime} 010 \mid L 0\right)\left(\ell_{1} 0 L 0 \mid C 0\right) W\left(1 \ell_{1}^{\prime} C \ell_{1} ; L k_{2}\right) \hat{k}_{I} W\left(k_{I} j_{1} I j_{2} ; j_{1}^{\prime} I\right) \\
& \times\left\{\begin{array}{ccc}
j_{1} & j_{1}^{\prime} & k_{I} \\
\frac{1}{2} & \frac{1}{2} & 1 \\
\ell_{1} & \ell_{1}^{\prime} & k_{2}
\end{array}\right\}\left\{\begin{array}{ccc}
1 & C & E \\
1 & k_{2} & k_{I} \\
2 & 1 & 1
\end{array}\right\} \sum_{\Gamma q_{I} \mu_{0} \mu_{3}}(-1)^{\epsilon}\left(C \Gamma 1 \mu_{0} \mid E \epsilon\right) Y_{C \Gamma}(\hat{\boldsymbol{R}}) \tau_{1 \mu_{0}}\left(\boldsymbol{\sigma}_{0}\right) \\
& \times\left(k_{I q_{I}} 1 \mu_{3} \mid E-\epsilon\right)\left\langle I, M_{f}\left|\tau_{k_{I} q_{I}}(\boldsymbol{I})\right| I, M_{i}\right\rangle\left[\nabla_{\mu_{3}} \chi_{\mu_{i}}^{(+)}(\boldsymbol{R}, k)\right] .
\end{aligned}
$$




\section{Appendix E}

\section{Nucleon-Nucleon Interaction \\ Parameters}

\begin{tabular}{|c||c|c|c|c|}
\hline & Central Singlet & Central Triplet & Spin-Orbit & Tensor \\
\hline \hline$V_{1}($ Even $)$ & $0.2955990 \mathrm{E}+04$ & $0.1892375 \mathrm{E}+04$ & $0.1491928 \mathrm{E}+04$ & $0.6585600 \mathrm{E}+05$ \\
$V_{1}($ Odd $)$ & $-0.2007265 \mathrm{E}+04$ & $0.1429753 \mathrm{E}+04$ & $-0.8840821 \mathrm{E}+03$ & $-0.1336309 \mathrm{E}+04$ \\
$1 / R_{1}$ & $0.4000000 \mathrm{E}+01$ & $0.4000000 \mathrm{E}+01$ & $0.4000000 \mathrm{E}+01$ & $0.6666667 \mathrm{E}+01$ \\
$V_{2}$ (Even) & $-0.1761237 \mathrm{E}+04$ & $-0.1182461 \mathrm{E}+04$ & $-0.5121186 \mathrm{E}+03$ & $-0.7824940 \mathrm{E}+04$ \\
$V_{2}$ (Odd) & $0.1318137 \mathrm{E}+04$ & $-0.4655380 \mathrm{E}+03$ & $-0.1173610 \mathrm{E}+03$ & $0.6631222 \mathrm{E}+03$ \\
$1 / R_{2}$ & $0.2500000 \mathrm{E}+01$ & $0.2500000 \mathrm{E}+01$ & $0.2500000 \mathrm{E}+01$ & $0.4000000 \mathrm{E}+01$ \\
$V_{3}$ (Even) & $0.1942531 \mathrm{E}+03$ & $0.1306984 \mathrm{E}+03$ & $0.1126246 \mathrm{E}+02$ & $0.3161026 \mathrm{E}+03$ \\
$V_{3}$ (Odd) & $-0.2821476 \mathrm{E}+03$ & $-0.5314848 \mathrm{E}+01$ & $0.5219727 \mathrm{E}+01$ & $-0.4232892 \mathrm{E}+02$ \\
$1 / R_{3}$ & $0.1818182 \mathrm{E}+01$ & $0.1818182 \mathrm{E}+01$ & $0.1818182 \mathrm{E}+01$ & $0.2500000 \mathrm{E}+01$ \\
$V_{4}$ (Even) & $-0.1509754 \mathrm{E}+02$ & $-0.1500969 \mathrm{E}+02$ & $0.0000000 \mathrm{E}+00$ & $-0.4909355 \mathrm{E}+02$ \\
$V_{4}$ (Odd) & $0.4404868 \mathrm{E}+02$ & $0.4833064 \mathrm{E}+01$ & $0.0000000 \mathrm{E}+00$ & $0.1440449 \mathrm{E}+02$ \\
$1 / R_{4}$ & $0.7142857 \mathrm{E}+00$ & $0.7142857 \mathrm{E}+00$ & $0.7142857 \mathrm{E}+00$ & $0.1428571 \mathrm{E}+01$ \\
\hline
\end{tabular}

Table E.1: The Yukawa parameters for the radial parts of the singlet/triplet, odd/even $v$ components of the $\mathrm{NN}$ interaction. The $V_{j}$ 's are the real strengths in $\mathrm{MeV}\left(\mathrm{MeV} . \mathrm{fm}^{-2}\right.$ for the tensor terms) and the $R_{j}$ 's are the range parameters in fm. 


\begin{tabular}{|c||c|c|c|c|}
\hline & Central Singlet & Central Triplet & Spin-Orbit & Tensor \\
\hline \hline$V_{1}$ (Even) & $0.5896003 \mathrm{E}+03$ & $0.1892375 \mathrm{E}+04$ & $0.7981813 \mathrm{E}+03$ & $0.5413153 \mathrm{E}+05$ \\
$V_{1}$ (Odd) & $-0.1098826 \mathrm{E}+04$ & $0.1429753 \mathrm{E}+04$ & $0.5356351 \mathrm{E}+01$ & $-0.1681731 \mathrm{E}+05$ \\
$1 / R_{1}$ & $0.4000000 \mathrm{E}+01$ & $0.4000000 \mathrm{E}+01$ & $0.4000000 \mathrm{E}+01$ & $0.6666667 \mathrm{E}+01$ \\
$V_{2}$ (Even) & $-0.3717699 \mathrm{E}+03$ & $-0.1182461 \mathrm{E}+04$ & $0.6659791 \mathrm{E}+02$ & $-0.6664950 \mathrm{E}+04$ \\
$V_{2}$ (Odd) & $0.1798922 \mathrm{E}+03$ & $-0.4655380 \mathrm{E}+03$ & $-0.1626521 \mathrm{E}+02$ & $0.1683316 \mathrm{E}+04$ \\
$1 / R_{2}$ & $0.2500000 \mathrm{E}+01$ & $0.2500000 \mathrm{E}+01$ & $0.2500000 \mathrm{E}+01$ & $0.4000000 \mathrm{E}+01$ \\
$V_{3}$ (Even) & $0.4781076 \mathrm{E}+02$ & $0.1306984 \mathrm{E}+03$ & $0.3068544 \mathrm{E}+02$ & $0.3289376 \mathrm{E}+03$ \\
$V_{3}$ (Odd) & $-0.7124365 \mathrm{E}+02$ & $-0.5314848 \mathrm{E}+01$ & $0.5499387 \mathrm{E}+01$ & $-0.7982533 \mathrm{E}+02$ \\
$1 / R_{3}$ & $0.1818182 \mathrm{E}+01$ & $0.1818182 \mathrm{E}+01$ & $0.1818182 \mathrm{E}+01$ & $0.2500000 \mathrm{E}+01$ \\
$V_{4}$ (Even) & $0.0000000 \mathrm{E}+00$ & $0.0000000 \mathrm{E}+00$ & $0.0000000 \mathrm{E}+00$ & $-0.6369222 \mathrm{E}+01$ \\
$V_{4}$ (Odd) & $0.0000000 \mathrm{E}+00$ & $0.0000000 \mathrm{E}+00$ & $0.0000000 \mathrm{E}+00$ & $0.1367362 \mathrm{E}+01$ \\
$1 / R_{4}$ & $0.7142857 \mathrm{E}+00$ & $0.7142857 \mathrm{E}+00$ & $0.7142857 \mathrm{E}+00$ & $0.1428571 \mathrm{E}+01$ \\
\hline
\end{tabular}

Table E.2: The Yukawa parameters for the radial parts of the singlet/triplet, odd/even $v$ components of the $\mathrm{NN}$ interaction. The $V_{j}$ 's are the imaginary strengths in $\mathrm{MeV}$ (MeV.fm ${ }^{-2}$ for the tensor terms) and the $R_{j}$ 's are the range parameters in $\mathrm{fm}$. 


\section{Bibliography}

[1] H. Feshbach, Annu. Rev. Nucl. Sci. 8, 49 (1958).

[2] H. Feshbach, in Nuclear Spectroscopy part B, edited by F. Ajzenberg-Selove (Academic Press, New York, 1960).

[3] K. T. R. Davies and G. R. Satchler, Nuclear Physics 53, 1 (1964).

[4] R. Wagner, P. D. Miller, T. Tamura, and H. Marshak, Phys. Rev. 139, B29 (1965).

[5] T. R. Fisher, D. C. Healey, and J. S. McCarthy, Nucl. Phys. A130, 609 (1969).

[6] C. J. Batty, Nucl. Phys. A178, 17 (1971).

[7] A. P. Stamp, Phys. Rev. 153, 1052 (1967).

[8] K. Nagamine, A. Uchida, and S. Kobayashi, Nucl. Phys. A145, 203 (1970).

[9] G. R. Satchler, Part. and Nuclei 1, 397 (1971).

[10] G. R. Satchler, Phys. Lett. 34B, 37 (1971).

[11] J. Dabrowski and P. Haensel, Phys. Lett. 42B, 163 (1972).

[12] T. R. Fisher, Phys. Lett. 35B, 573 (1971).

[13] A. H. Hussain and H. S. Sherif, Phys. Rev. C 8, 518 (1973).

[14] W. Heeringa, H. Postma, H. Dobiasch, R. Fisher, H. O. Klages, R. Maschuw, and B. Zeitnitz, Phys. Rev. C 16, 1389 (1977).

[15] B. von Przewoski, P. D. Eversheim, F. Hinterberger, U. Lahr, J. Campbell, J. Gotz, M. Hammans, R. Henneck, G. Masson, I. Sick, and W. Bauhoff, Nucl. Phys. A528, 159 (1991).

[16] J. P. Soderstrum, C. R. Gould, D. G. Haase, N. R. Roberson, M. B. Schneider, and L. W. Seagondollar, Phys. Rev. C 45, 326 (1992). 
[17] F. Petrovich, R. J. Philpott, A. W. Carpenter, and J. A. Carr, Nucl. Phys. A425, 609 (1984).

[18] M. M. Nagadi, G. L. Weisel, R. L. Walter, J. P. Delaroche, and P. Romain, Phys. Rev. C 70, 064604 (2004).

[19] H. S. Sherif, in Proc. 4th Int. Symp. on polarization phenomena in nuclear reactions, Zurich., edited by W. Gruebler and V. Konig (Birkhauser, Basel, 1976).

[20] T. L. McAbee, W. J. Thompson, and H. Ohnishi, Nucl. Phys. A509, 39 (1990).

[21] T. L. McAbee, Ph.d. dissertation, University of North Carolina, Chapel Hill, North Carolina, USA, 1986.

[22] H. Wolfenstein, Annu. Rev. Nucl. Sci. 6, 43 (1956).

[23] H. S. Sherif and A. H. Hussain, Phys. Lett. 41B, 465 (1972).

[24] C. J. Batty and C. Tschalar, Nucl. Phys. A143, 151 (1970).

[25] P. Catillon, in Polarization Phenomena in Nuclear Reactions, p657, edited by H. H. Barschall and W. Haeberli (Univ. of Wisconsin Press, Madison, Wisc., 1970).

[26] K. Katori, T. Nagata, O. Mikoshiba, and S. Kobayashi, in Polarization Phenomena in Nuclear Reactions, p638, edited by H. H. Barschall and W. Haeberli (Univ. of Wisconsin Press, Madison, Wisc., 1970).

[27] J. Birchall, H. E. Conzett, J. Arvieux, W. Dahme, and R. M. Larimer, Phys. Lett. 53B, 165 (1974).

[28] R. A. Hardekopf and D. D. Armstrong, Phys. Rev. C 13, 900 (1976).

[29] W. G. Weitkamp, W. Grüebler, V. König, P. A. Schmelzbach, R. Risler, and B. Jenny, Nucl. Phys. A311, 29 (1978).

[30] H. S. Sherif, Phys. Rev. C 19, 1649 (1979).

[31] W. J. Thompson, Phys. Lett. 62B, 245 (1976).

[32] J. S. Blair, M. P. Baker, and H. S. Sherif, Phys. Lett. 60B, 25 (1975).

[33] G. Roy, H. S. Sherif, E. D. Cooper, L. G. Greeniaus, G. A. Moss, J. Soukup, G. M. Stinson, R. Abegg, D. P. Gurd, D. A. Hutcheon, R. Liljestrand, and C. A. Miller, Nucl. Phys. A442, 686 (1985). 
[34] T. Nakano, M. Nakamura, H. Sakaguchi, M. Yosoi, M. Ieiri, H. Togawa, S. Hirata, O. Kamigaito, H. M. Shimizu, M. Iwaki, Y. Nakai, S. Kobayashi, T. Noro, and H. Ikegami, Phys. Lett. B 240, 301 (1990).

[35] A. C. Betker, S. Chang, E. J. Stephenson, A. D. Bacher, S. M. Bowyer, W. A. Franklin, J. Liu, C. Olmer, D. L. Prout, S. P. Wells, S. W. Wissink, C. Yu, R. A. Lindgren, H. Baghaei, V. Gladyshev, J. A. Carr, S. K. Yoon, F. Petrovich, B. L. Clausen, and J. Lisantti, Phys. Rev. C 71, 064607 (2005).

[36] E. Bleszynski, M. Bleszynski, and C. A. Whitten, Jr, Phys. Rev. C 26, 2063 (1982).

[37] P. R. Lewis, G. G. Shute, B. M. Spicer, R. S. Henderson, R. Abegg, D. Frekers, O. Häusser, K. P. Jackson, C. A. Miller, and S. Yen, Nucl. Phys. A532, 583 (1991).

[38] D. Koning, Nucl. Phys. A317, 175 (1979).

[39] F. C. Barker, Aust. J. Phys. 34, 7 (1981).

[40] S. Cohen and D. Kurath, Nuclear Physics 73, 1 (1965).

[41] T. S. H. Lee and D. Kurath, Nuclear Physics 73, 1 (1965).

[42] D. M. Brink and G. R. Satchler, Angular Momentum, $3^{\text {rd }}$ ed. (Oxford University Press, Oxford, UK, 1993).

[43] D. J. Hooton and R. C. Johnson, Nucl. Phys. A175, 583 (1971).

[44] G. R. Satchler, Direct Nuclear Reactions (Oxford University Press, New York, 1983).

[45] M. L. Goldberger and K. M. Watson, Collision Theory (Dover Pub. Inc., New York, 2004).

[46] J. S. Al-Khalili, Ph.d. dissertation, University of Surrey, Guildford, UK, 1989.

[47] J. S. Al-Khalili and R. C. Johnson, Nucl. Phys. A546, 622 (1992).

[48] M. E. Rose, Elementary Theory of Angular Momentum (Dover Publications, New York, 1995).

[49] M. Abramowitz and I. A. Stegun, Handbook of Mathematical Functions, $9^{\text {th }}$ ed. (Dover Pub. Inc., New York, 1972).

[50] H. Feshbach, Ann. Phys. 5, 357 (1958).

[51] H. Feshbach, Ann. Phys. 19, 287 (1962). 
[52] D. F. Jackson, Nuclear Reactions (Methuen \& Co Ltd., London, UK, 1970).

[53] R. Crespo, R. C. Johnson, and J. A. Tostevin, Phys. Rev. C 44, R1735 (1991).

[54] R. Crespo, R. C. Johnson, and J. A. Tostevin, Phys. Rev. C 46, 279 (1992).

[55] G. W. Greenlees, G. J. Pyle, and Y. C. Tang, Phys. Rev. 171, 1115 (1968).

[56] R. C. Barrett and D. F. Jackson, Nuclear Sizes and Shapes (Clarendon Press, Oxford, 1977).

[57] G. R. Satchler and W. G. Love, Phys. Rep. 55, 183 (1979).

[58] A. deShalit and I. Talmi, Nuclear Shell Theory (Academic Press, New York, 1963).

[59] R. B. Taylor, N. R. Fletcher, and R. H. Davis, Nucl. Phys. A65, 318 (1965).

[60] J. C. Hafele, C. B. Fulmer, and F. G. Kingston, Phys. Lett. 31B, 17 (1970).

[61] C. B. Fulmer and J. C. Hafele, Phys. Rev. C 7, 631 (1973).

[62] G. H. Rawitscher, Phys. Rev. C 6, 1212 (1972).

[63] W. G. Love, Nucl. Phys. A226, 319 (1974).

[64] F. Petrovich, D. Stanley, L. A. Parks, and P. Nagel, Phys. Rev. C 17, 1642 (1978).

[65] P. Ring and P. Schuck, The Nuclear Many-Body Problem (Springer-Verlag, Berlin, Germany, 2004).

[66] T. Hamada and I. D. Johnston, Nuclear Physics 34, 382 (1962).

[67] R. V. Reid, Jr., Ann. Phys. (N.Y.) 50, 411 (1968).

[68] K. A. Brueckner, C. A. Levinson, and H. M. Mahmoud, Phys. Rev. 95, 217 (1954).

[69] K. A. Brueckner, Phys. Rev. 96, 508 (1954).

[70] K. A. Brueckner, Phys. Rev. 97, 1353 (1955).

[71] F. Sammarruca, E. J. Stephenson, and K. Jiang, Phys. Rev. C 60, 064610 (1999).

[72] A. L. Fetter and J. D. Walecka, Quantum Theory of Many-Particle Systems (Dover Pub. Inc., New York, 2003).

[73] G. Bertsch, J. Borysowicz, H. McManus, and W. G. Love, Nucl. Phys. A284, 399 (1977). 
[74] M. E. Brandan and G. R. Satchler, Phys. Rep. 285, 143 (1997).

[75] M. Lacombe, B. Loiseau, J. M. Richard, R. V. Mau, J. Côté, P. Pirès, and R. de Tourreil, Phys. Rev. C 21, 861 (1980).

[76] N. Anantaraman, H. Toki, and G. F. Bertsch, Nucl. Phys. A398, 269 (1983).

[77] R. Machleidt and G. Q. Li, Phys. Rep. 242, 5 (1994).

[78] W. G. Love and M. A. Franey, Phys. Rev. C 24, 1073 (1981).

[79] W. G. Love and J. R. Satcher, Nucl. Phys. A159, 1 (1970).

[80] E. J. Stephenson, private communication, 2009.

[81] A. deShalit and H. Feshbach, Theoretical Nuclear Physics, vol. 1: Nuclear Structure (Wiley, New York, 1974).

[82] Y. C. Tang, M. LeMere, and D. R. Thompson, Phys. Rep. 47, 167 (1978).

[83] M. LeMere and Y. C. Tang, Phys. Rev. C 19, 391 (1979).

[84] F. Petrovich, H. McManus, V. A. Madsen, and J. Atkinson, Phys. Rev. Lett. 22, 895 (1969).

[85] M. Golin, F. Petrovich, and D. Robson, Phys. Lett. 64B, 253 (1976).

[86] G. R. Satchler and W. G. Love, Phys. Lett. 65B, 415 (1976).

[87] R. C. Johnson, private communication, 2009.

[88] A. R. Edmonds, Angular momentum in quantum mechanics (Princeton University Press, New Jersey, 1960).

[89] F. D. Santos, Ph.d. dissertation, University of Surrey, Guildford, Surrey, UK, 1968.

[90] D. A. Varshalovich, A. N. Moskalev, and V. K. Khersonskii, Quantum Theory of Angular Momentum (World Scientific, Singapore, 1988).

[91] I. Talmi, Simple Models of Complex Nuclei. (Harwood Academic Publishers, Switzerland, 1993).

[92] S. Sengupta, Nuclear Physics 30, 300 (1962).

[93] A. Cichocki, J. Dubach, R. S. Hicks, G. A. Peterson, C. W. de Jager, H. de Vries, N. Kalantar-Nayestanaki, and T. Sato, Phys. Rev. C 51, 2406 (1995). 
[94] H. A. Jahn and J. Hope, Phys. Rev. 93, 318 (1954).

[95] R. C. Johnson, Ph.d. dissertation, University of Manchester, Manchester, UK, 1961.

[96] G. B. Arfken and H. J. Weber, Mathematical Methods for Physicists, $5^{\text {th }}$ ed. (Academic Press, Orlando, FL, 2001). 